\title{
On Fatigue Life Under Stationary Gaussian Random Loads (A)
}

Talreja, $\mathbf{R}$.

Published in:

Acoustical Society of America. Journal

Link to article, DOI:

$10.1121 / 1.1982256$

Publication date:

1973

Document Version

Publisher's PDF, also known as Version of record

Link back to DTU Orbit

Citation (APA):

Talreja, R. (1973). On Fatigue Life Under Stationary Gaussian Random Loads (A). Acoustical Society of America. Journal, 53(1), 309-309. https://doi.org/10.1121/1.1982256

\section{General rights}

Copyright and moral rights for the publications made accessible in the public portal are retained by the authors and/or other copyright owners and it is a condition of accessing publications that users recognise and abide by the legal requirements associated with these rights.

- Users may download and print one copy of any publication from the public portal for the purpose of private study or research.

- You may not further distribute the material or use it for any profit-making activity or commercial gain

- You may freely distribute the URL identifying the publication in the public portal

If you believe that this document breaches copyright please contact us providing details, and we will remove access to the work immediately and investigate your claim. 


\title{
PROGRAM OF
}

\section{The Eighty-Fourth Meeting of the Acoustical Society of America}

\author{
Carillon Hotel • Miami Beach, Florida $\quad$ - 28 November-1 December 1972
}

TUESDAY, 28 NOVEMHER 1972

Burgundy East, 9:00 A.M.

\author{
Session A. Physiological Acoustics I : Anatomy and Mechanics \\ Russell R. Pfeiffer, Chairman \\ Department of Electronics, Washington University, St. Louis, Missouri 63130
}

Contributed Papers (10 minutes)

\begin{abstract}
A1. Relative Development of Inner, Middle, and Erternal Ear of The Rat. Ruth G. Rorins, Department of Otolaryngology, University of Oregon Medical School, Porlland, Oregon 97201.-The maturation of the cochlea is of ten emphasized when correlating physiological, behavioral, and anatomical data with hearing inception, but the contribution of the conductive mechanism must also be considered. This study compares the development of all parts of the ear in the rat from 11 days postconception to 1 month postpartum. Although the hair cells are distinguishable at birth, the remainder of the organ of Corti is poorly differentiated. The external auditory meatus is closed, the middle ear is filled with a gelatinous mass, the ossicles are cartilagenous, and the stapes is continuous with the otic capsule. Maturation of the cochlea is seen first in the basal coil at eight days postpartum, evidenced by the appearance of the tunnel of Corti, the extracellular spaces between the hair cells, and nerve fibers crossing the tunnel. At this time, however, the external meatus is still closed, some gelatinous material is still retained in the middle ear. The stapedio-vestibular "ligament" is composed of cells rather than fibers, limiting its flexibility. Structural differentiation of the cochlea proceeds from base to apex, being completed by the 17 th day. The annular ligament does not lose its cellular component until the 21 st day.
\end{abstract}

A2. Auditory Sensitivity in the Tadpole (Rana catesbeiana). B. A. Weiss and B. H. Stuart, Psychology Department, Drexel University, Philadelphia, Pennsyluania 19104, AND W. F. Strother, Psychology Department, Princeton University, Princelon, New Jersey 08540.-Auditory sensitivity in six bullfrog tadpoles was investigated by electrophysiological techniques. Tonal stimulus intensities that generated a standard potential of $0.1 \mu \mathrm{V}$ in the inner ear showed a hearing range from 100 to $4000 \mathrm{~Hz}$ with maximum sensitivity near $1 \mathrm{dyn} / \mathrm{cm}^{2}\left(0.1 \mathrm{~N} / \mathrm{m}^{2}\right)$ in the lower portion of the range. The data also indicated that tadpoles lack the place mechanism of adult frogs and thus place as a mechanism appeared at the amphibian metamorphosis in the course of the evolution of vertebrate hearing.

A3. Auditory Sensitivity in the Agouti (Dasyprocta punctata). G. M. Hartig, W. M. Bayram, A. Poynton III, and E. A. Peterson, Division of Auditory Research Department of Otolaryngology, School of Medicine, Miami. Florida 33152.A conditioned suppression technique has been applied to five agoutis and behavioral thresholds measured. Each animal was water deprived and allowed to drink only in the experimental situation. Training was effected by pairing audible tone pulses with shock until the subject learned to cease drinking reliably in the presence of a signal. Auditory thresholds were then assessed at octave intervals. Maximum sensitivity occurred at $8 \mathrm{kHz}\left(0 \mathrm{~dB}\right.$ re $\left.2 \times 10^{-1} \mathrm{dyn} / \mathrm{cm}^{2}\right)$, a high frequency cutoff at $64 \mathrm{kHz}$, and a low-frequency cutoff at $500 \mathrm{~Hz}$. Low-frequency sensitivity may have been impaired by masking or TTS resulting from the lond licking noises produced by this species, although control studies have not verified this hypothesis. It is the authors' opinion that hearing in the Agouti is oriented toward the higher frequencies despite the presence of certain low-frequency structures such as the large bulla. The observed high-frequency bias supports previous findings regarding this animal's basilar membrane.

A4. Sound Pressure Transformation from the Free Field to the Eardrum. E. A. G. SHAw, Division of Physics, National Council of Canada, Otlawa, KIA OSI, Canada.-The sound pressure transformation by the human head and external ear has been the subject of many measurements over a long period of time. The average acoustic properties of the external ear are now established sufficiently well that diverse data can be brought into a common set of reference Irames. Data from 10 studies in five countries are presented in terms of eardrum response at eight azimuthal angles as functions of frequency from 0.2 to $12 \mathrm{kHz}$. Data from the same pool are also presented in terms of azimuthal variations and interaural level differences at 18 frequencies. Where there is lack of agreement between studies, the presentation often indicates the probable causes. A critical examination of all the data leads to self-consistent families of average response at the eardrum position as a function of frequency and angle of incidence in the azimuthal plane. An extension of the method should provide useful estimates of average response at angles of incidence not on the horizontal plane.

A5. Aural Noise as a Function of the Cardiac Cycle. D. R. Soderquist, J. W. Lindsey, and M. R. Harter, Department of Psychology, University of North Carolina at Greensboro, Greensboro, North Carolina 27412.--Investigators have suggested several internal noises that affect absolute thresholds (e.g., heart sounds, circulatory pulsations, muscle tremors). Furthermore, it has been suggested that blood flow may 
modulate aural noise in the external canal. Since meatal blood vessels cannot expand outward due to bone encasing the canal, an inward expansion is suggested which induces an increase in aural noise by decreasing meatal volume under the earphone. In short, the meatal pulsation hypothesis (MPH) suggests a modulation of aural noise as a function of blood flow through the ear canal. This study examines the MPH under three conditions (tight and normal fitting supraaural cushions and an insert earmold) as a function of the cardiac cycle. Three dependent measures were obtained, viz., EKG, blood flow at the earlobe, and pressure changes within the ear canal (positive pressure indicating, according to the MPH, an inward expansion of the blood vessels). The EKG $R$-wave keyed a signal averaging computer which in turn averaged the data over 32 separate heartbeats. Results indicate: (1) a maximum positive pressure approximately 225-250 msec after the $\mathrm{R}$-wave with rapid decreases prior to and immediately after the maximum (tight fitting earphones); (2) no systematic pressure change with normally fitting earphones; (3) an inverse relationship between blood flow at the earlobe and pressure change within the meatus. The maximum negative pressure occurring near $225-250$ msec after the R-wave.

A6. Morphological Correlates of Asymptotic Temporary Threshold Shift in Chinchillas. David LIM AND William Melnick, Depariment of Otolaryngology, The Ohio State University, Columbus, Ohio 43210.--Using the scanning and transmission electron microscopes, an attempt was made to correlate morphological and behavioral data from conditioned chinchillas following their exposure to a presumably subtraumatic level of noise that produced asymptotic TTS. Varying degrees of morphological change in the sensory cells, which could be attributed to the noise exposures, were observed in these animals. Changes included giant cilia formation, sensory cell debris, cellular swelling, and scar formation. Changes also appeared in the stria vascularis. These morphological changes were most pronounced in the apical turn of the cochlea. Our preliminary data appear to support the idea that sensory cell degeneration occurs even after exposure to a noise level considered subtraumatic and which apparently does not produce permanent changes in behavioral threshold hearing levels.

A7. Anatomical Correlates of a Temporary Shift in the Threshold of Hearing. B. A. BoHNE, Depariment of Otolaryngology, Washington University and Central Institute for the Deaf, St. Louis, Missouri 63110.-When chinchillas are continuously exposed for more than $24 \mathrm{~h}$ to an octave band of noise centered at $500 \mathrm{~Hz}$, the behavioral TTS for $715-\mathrm{Hz}$ tone stabilizes at an asymptotic value proportional to band level [Carder and Miller, Trans. Amer. Acad. Ophthal. Otolaryng. 75, 1346 (1971)]. Recovery to normal thresholds requires from two to seven days. Chinchillas were exposed to this same noise for two days at $65,75,85$, or $95 \mathrm{~dB}$ SPL. The organ of Corti was immediately fixed and prepared as a flat, whole-mount specimen in plastic suitable for both phase contrast and electron microscopy. Other ears exposed at $95 \mathrm{~dB}$ SPL were allowed to recover 7,28 , or 70 days before fixation. The number of cisternae in the peripheral membrane system of many outer hair cells was increased from three to six to as many as 30 . Similar membranes appeared as whorls within the cells. The proportion of altered cells was greatest in upper second and lower third turns and increased with exposure levels. Recovery toward normal membrane configuration was evident at seven days and only minor variations remained after 28 and 70 days.

A8. Guided Interfacial Waves with Applications to Cochlear Mechanics. R. J. Clifton, Professor of Engineering, Brown Universily, Providence, Rhode Island.-Waves are considered which propagate along a membrane separating two compressible, inviscid fluids. Capillary forces and membrane tension are assumed to provide the stiffness for the membrane. Dispersion relations are derived for cases of (i) an unbounded region, (ii) a channel for which the particle velocity at the sides is perpendicular to the interface, and (iii) a slit for which the particle velocity at the sides is parallel to the interface. The solution for the latter case is obtained by first solving the problem of diffraction of interfacial waves by a rigid plate in the plane of the interface. Results of case (iii) are used to examine the importance of interfacial waves for wave propagation in the cochlea. Preliminary evidence indicates that such waves may be of primary importance for frequencies in the upper part of the audible range.

A9. Measurements of the Transient Response of the Basilar Membrane Using the Mössbauer Technique. L. Robles, W. S. Rhode, AND C. D. GEISLER, Laboratory of Neurophysiology and Laboratory Compuler Facility, University of ' W'isconsin 53706.-Measurements were performed on 25 squirrel monkeys, using an experimental preparation identical to the one used by Rhode to measure frequency response [J. Acoust. Soc. Amer. 49, 1218-1231 (1971)]. The stimuli used were acoustic clicks, about $150 \mu \mathrm{sec}$ in duration, presented in sequences of 100000 to 400000 . Clicks with different amplitudes were used, and the responses of the basilar membrane at a point in the basal turn and of the umbo were measured. The results indicate that the click response of the basilar membrane at the points measured is a lightly damped oscillation with a natural frequency between 6 and $8 \mathrm{kHz}$. There is an initial part of the response that has a faster decay and a later part that has an extremely slow rate of decay. This later part of the response displays a nonlinear behavior for changes in amplitude of the stimulus. Even when the general features of the vibratory pattern were stable for a period of hours, there was usually a marked decrease with time in the number of cycles which could be observed. [Supported by NiH Grants.]

A10. Eardum Displacement Following Stapedius Muscle Contraction. A. Yonovitz and J. D. Harris, U. S. Naval Submarine Medical Center, Submarine Base New London. Groton, Connecticut 06340.- Simultaneous monitoring in human subjects on the same ear of eardrum displacement by tympanomanometry, and acoustical impedance by the Madsen bridge, provided information concerning contraction of the stapedius muscle and its effect on eardrum displacement. Extensive control procedures were employed to elicit only the stapedius: lower-intensity auditory stimulation, electrocutaneous stimulation of the homolateral external ear canal, and anesthetization of nerves leading to the tensor tympani. (1) Extremely small biphasic and monophasic eardrum movements were seen in the stapedious-only ear to auditory and electrocutaneous stimulation; the form of the response was much less predictable to auditory stimulation. (2) At higher sound intensities, relatively large inward and biphasic movements of the eardrum occurred in the normal ear, unquestionably resulting from contraction of the tensor tympani. These results were further validated in a group of stapedetomized ears, without the stapedius but with normal tensor tympani. (3) Biphasic responses did not occur in the tensor 
tympani-only ear only monophasic inward responses. (4) Upon air-jet stimulation to the orbit of the eye, these subjects had an accentuated tensor response in that large inward movements of the eardrum occurred as compared to those in normal ears, suggesting that there is an alteration of the tensor response by the presence of the stapedius muscle. Estimates of the actual eardrum displacements were calculated based on a model of the external ear canal and eardrum.

\section{A11. Abstract withdrawn.}

A12. Tympanic Muscle Effects on Middle-Ear Transfer Characteristic. Alfred L. Nutrall, Kresge Hearing Research Institule, University of Michigan, Ann Arbor, Michigan 48104. - The change in the magnitude and phase of sound transmission through the guinea-pig open-bulla middle ear is measured when two levels of independent, isotonic, tympanic muscle contraction are effected by direct electric stimulation of the muscle bodies. Both middle-ear muscles attenuate the passage of low-frequency vibration giving the largest attenuation for frequencies below $300 \mathrm{~Hz}$, the tensor tympani producing $28 \mathrm{~dB}$ maximum attenuation, the stapedius producing $10 \mathrm{~dB}$. In the $1-$ to $3-\mathrm{kHz}_{2}$ frequency range, both muscles are capable of generating an apparent gain in transmission: the tensor tympani giving a gain of $5 \mathrm{~dB}$ at $2.5 \mathrm{kHz}$ for maximum contraction, the stapedius $0.5 \mathrm{kHz}$ at $2.5 \mathrm{kHz}$ for maximum contraction. The phase shifts for all contraction cases were leading phase functions. Changes in the magnitude and phase of the transmission are modeled by a second-order low-pass system where the break-frequency increases and the damping ratio and low-frequency magnitude decrease with muscle contraction. The transfer function with maximum tensor contraction had a break Irequency of $2.5 \mathrm{kHz}$ with a damping ratio of 0.31 , while the stapedius maximum contraction gave a breakfrequency of $1.57 \mathrm{kHz}$ and a damping ratio of 0.71 . These transmission changes are accounted for the most part, in the model, by a decrease in compliance of the middle ear, but there are also increases in resistance and inertance over the flaccid muscle state.

A13. Animal Exposure to Infrasound. DANIEL L. Johnson, 6570th Aerospace Medical Research Laboratory, Wright-Patterson Air Force Base, Ohio 45433.--The AMRL dynamic pres- sure chamber (a hydraulic device that produces intense infrasound) recently became operational. Preliminary to human whole body exposures, a variety of experiments with animals are being performed in order to evaluate physiological limits. A monkey, a young baboon, and six dogs of various sizes anesthetized with pentobarbital were exposed from 1 to $4 \mathrm{~h}$ at the maximum leveis the chamber is capable of producing (172.5 dB from 1 to $8 \mathrm{~Hz}$ and falling off $7.6 \mathrm{~dB} / \mathrm{oct}$ to $158 \mathrm{~dB}$ at $30 \mathrm{~Hz}$ ). EKG and respiration rate (via chest impedance measurements) were recorded. The only observed physiological effects were a decline in respiration rate and some reddening of the tympanic membrane. Except for three of the dogs, these animals were then given exposures in which they were not anesthetized. Exposure levels were gradually increased until all animals received the maximum chamber SPL for 1-14 h. Their behaviors were subjectively observed to be normally calm and occasionally restless. At no time did the animals appear in discomfort or show signs of nystagmus or dizziness. The lack of adverse results of these high exposure levels will be discussed.

\section{A 14. Real-Time Observation of Submicroscopic Vibration by} Stroboscopic Holography. Shyam M. KhanNa AND JUERGeN Tonndorf, Columbia University, New York 10032.-Timeaveraged holography has been successfully employed for obtaining high-contrast vibratory patterns of the tympanic membrane [Khanna and Tonndorf, J. Acoust. Soc. Amer. 4I, 1904 (1972)]. Nevertheless, this technique has two restrictions: (1) the time delay due to the photographic processing of the holographic plates and (2) its essential inability to give phase information. Attempts to employ real-time holography [Tonndorf et al., J. Acoust. Soc. Amer. 46, 106 (1969)] for the same purpose failed because of (a) its inherent low fringe contrast and (b) its sensitivity to slow, quasi-dc, membrane motions. Real-time holography that includes strobing [Archbold and Ennos, Nature 217, 942 (1968)] markedly improves the image contrast, as will be denonstrated on an earphone diaphragm. It also gives both amplitude and phase information. Additional phase modulation [Aleksoff, Appl. Phys. Lett. 14, $23(1969)]$, as will also be shown, permits removal of the $180^{\circ}$ phase ambiguity. This latter combination holds good promise for application to measurements on biological membranes. [Supported by several NIH grants.]

TUESDAY, 28 NOVEMBER 1972

Silver Chimes East, 9:00 A.м.

\author{
Session B. Speech Communication I : Vocal Tract Models and Physiology
}

Sandra Hamlet, Chairman

Speech Department, University of Maryland, Baltimore, Maryland

\title{
Contributed Papers (7 minutes)
}

B1. Direct Determination of Input Impedance Singularities from Speech for Obtaining the Vocal Tract Area Function. 11. Wakita, Speech Communications Research Laboralory $(S C R L)$, Santa Barbara, California 93101.-This method is based on the acoustic tube model of the vocal tract in which the tube is divided into a finite number of sections with equal lengths. The volume velocity is defined in each section. Input impedance at the front end of the tube (corresponding to the lips) can be computed as the ratio of the pressure and the volume velocity there, providing that the volume velocity and the pressure are continuous at each boundary between two adjacent sections. This input impedance is shown to be of the form $D(z) / G(z)$, where $D(z)$ and $G(z)$ are polynomials in $z^{-1}$. It is also shown that $D(z)$ and $G(z)$ are con- 
structed as a difference and a sum, respectively, of two polynomials whose coefficients can be determined from the reflection coefficients of the acoustic pube model. Since a method for obtaining the reflection coefficients from the speech wave is already established [H. Wakita, SCRL Monogr. No. 9 (July 1972)], $D(z)$ and $G(z)$ can thus be obtained from the speech wave. Input impedance singularities obtained with area functions for five Russian vowels by Fant were in very good agreement with Mermelstein's results [J. Acoust. Soc. Amer. 41, 1283-1294 (1967)]. [The Office of Naval Research supported this work.]

B2. Experimental Determination of the Area Function of a Lossy, Dynamically Varying Vocal Tract. M. M. Sondhl, Bell Laboratories, Murray Hill, New Jersey 07974.-During the last year we have made acoustic impulse-response measurements at the lips while the vocal tract is moved as in normal speech. The experiment will be described and moves will be shown of the area functions reconstructed from the measurements. A method will be presented for estimating the mechanical impedance of the walls of the vocal tract. It will be shown that the reconstructed areas are significantly improved if the walls, instead of being assumed rigid, are assumed to have this estimated impedance.

B3. Models of Articulatory Dynamics. C. H. Coker and C. P. Browman, Bell Laboralories, Murray Hill, New Jersey 07974. - We present comparisons between the x-ray data of R. A. Houde [PhD Thesis, Univ. of Michigan (1967)] and actions of a dynamic articulatory model. The data can be fitted in two ways: (1) a model with minimum number of degrees of freedom, but with time-varying "characteristic filters" (Coker and Fujimura, J. Acoust. Soc. Amer. 40, 11 (A) (1966)]; or (2) one in which different speeds of an articulator are produced by additional "characteristic variables" that would be redundant in the static case. The phenomenon that the constriction tends to move forward during /g/ was suggested by Houde to result from pressure buildup in the enclosed cavity. We treat the effect as a peculiarity in "programming" of $/ \mathrm{g} /$. Horizontal motions toward $/ \mathrm{g} /$ from front vowels are commanded 50 msec early, from back vowels, 50 msec late, thus producing a circular Lissajous pattern of motion. The dilemma of cause and effect (whether continued voicing forces tongue-body movement, or whether movement is explicitly commanded, to sustain voicing) leads to interesting questions when we attempt to generalize to other phonemes. Does circular motion occur in nasals and voiceless stops as well as $/ \mathrm{g} /$ ? Does the tendency for $/ \mathrm{g} /$ to devoice in some languages derive from differences in tongue control? These and other topics are discussed.

B4. Nonlinear Inverse Filtering. Martin RothenberG, Department of Electrical and Computer Engineering, Syracuse University, Syracuse, New York 13210.--It has been shown by Flanagan and Landgraf [IEEE Trans. Audio Electroacoust. 16, No. 1, 57-64 (1968)] that in the transformation between glottal area and glottal volume velocity during voiced vowels, the effect of the interaction between the acoustic impedance of the glottis and that of the supraglottal vocal tract cannot always be neglected. When there is such interaction, the glottal-supraglottal acoustic system must be considered a nonlinear system with time-varying parameters. Thus, any inverse-filtering process designed to extract the glottal area wavelorm from measurements of supraglottal pressure or air flow must also be nonlinear. This paper reports initial efforts to determine the conditions under which a nonlinear inverse-filtering process exists that will yield a function directly related to glottal area from recordings of oral volume velocity, and how such a nonlinear inverse filter could be implemented.
B5. Mathematical Considerations in Digital Simulations of the Vocal Tract. M. R. Portnoff and R. W. Schafer, Bell Laboratories, Murray Hill, New Jersey 07974.-The formulation of a digital simulation of speech production involves two basic steps. The first step is a mathematical representation of the physics of sound generation and propagation in the vocal tract. These processes are of a continuous nature and are generally represented mathematically by differential equations. The second step is the transformation of the mathematical description of the physical processes into a discretetime model in the form of a set of difference equations suitable for implementation on a digital computer. As a result of this second step, a number of mathematical issues arise that are unrelated to the physics of speech production. These issues often receive insufficient consideration. In fact, it is easily shown that intuitively reasonable discrete-time approximations often have undesirable characteristics. The theory of digital signal processing provides a means for understanding these issues and for formulating accurate digital simulations. This approach is illustrated by a discussion of a simulation involving a soft-walled nonuniform acoustic tube excited by a two-mass model of the vocal cords.

B6. Further Theoretical and Experimental Bases for Quantal Places of Articulation for Consonants. KenNeth N. Stevens, Research Laboratory of Electronics and Department of Electrical Engineering, Massachusetts Inslitute of Technology, Cambridge, Massachusetts 02139 .- - Previous theoretical studies of the acoustic behavior of a vocal-tract model consisting of a tube with a narrow constriction located at different points along its length have suggested that there is a series of constriction positions for which the sound output has well-defined quantal attributes. It has been hypothesized that these positions correspond to places of articulation that are used to produce consonants in a variety of languages. Recent work has refined the theoretical basis on which these places of articulation are predicted, and has shown that the shape of the constriction as well as its positions plays a role in determining the distinctive acoustic attributes of the output, particularly for consonants produced with the tongue blade. Furthermore, data on the acoustic properties of stop and fricative consonants from several different languages have been collected and have been shown to be consistent with the theoretical framework. The predicted places of articulation account for consonants in the pharyngeal, uvular, and velar regions, and several consonant classes in the dental-alveolar-palatal regions. [Work supported in part by National Institutes of Health and in part by the Office of Naval Research.]

B7. Physiological Aspects of Certain Laryngeal Features in Stop Production. Hajime Hirose," Leigh Lisker, $\dagger$ and Arthur S. Abramson, $¥$ Haskins Laboratories, New Haven, Connecticut 06510.-Multichannel EMG recordings were made of the instrinsic and extrinsic laryngeal muscles during production of intervocalic labial stops of five phonetic types: explosive voiced inaspirate, implosive voiced inaspirate, voiced aspirate, voiceless inaspirate, and voiceless aspirate. The recorded data, computer processed to obtained average EMG values, indicate that abductor and adductor muscle groups follow coordinated patterns of activity corresponding to opening and closing of the glottis. In particular, the interarytenoid and posterior cricoarytenoid muscles showed reciprocal patterns both in degree and timing of activity. In addition, the sternohyoid showed marked activity for the implosive stop, presumably correlated with an abrupt lowering of the larynx. In a second experiment using the same speaker and the same syllables, motion pictures of the glottis were taken by means of a flexible fiberscope. The overall conclusion suggested is that active adjustments of the glottis in terms of coordinated muscle activity, and the timing of this activity 
relative to supraglottal events, are the decisive factors by which the various stop consonant types are differentiated. [This research was supported by the National Institute of Dental Research through an NIH grant]

* Also University of Tokyo, Tokyo, Japan.

† Also University of Pennsylvania, Philadelphia, Pa. 19104. $\ddagger$ Also University of Connecticut, Storrs, Conn. 06268.

B8. Stop Consonant Voicing and Pharyngeal Cavity Size. Fredericka B. Berti* and HajIME Hirose, † Haskins Laboratories, New Haven, Connecticul 06510.-Aerodynamic forces require a vocal tract volume increase subsequent to oral cavity occlusion if voicing is to proceed through stop consonant closure. Electromyographic recordings of pharyngeal musculature were obtained for three subjects during the production of the six stop consonants of English in controlled phonetic environments. In addition, simultaneous electromyographic recordings and fiberscopic motion pictures were obtained for one subject producing a subset of the total utterance set. Each subject shows differences in the pattern of cavity enlargement for the voiced stops. One subject shows increased levator palatini and sternohyoid activity, and demonstrates greater velar elevation (determined from the motion pictures) for voiced stops as compared with voiceless stops. A second shows decreased activity of the pharyngeal wall musculature along with increased activity of the sternohyoid for the voiced stop cognates. The third subject exhibits a composite of the activity patterns of the first two subjects. The data support earlier suggestions that pharyngeal expansion must be due, at least in part, to positive muscle activity. They also indicate that there may be intersubject variation of actuating mechanisms while the articulations are perceived as equal. [This research was supported by the National Institute of Dental Research, through an NIH grant.]

* Also Montclair State College, Upper Montclair, N. J. 07043, and The Graduate School, The City University of New York, N. Y. 10036.

† Also University of Tokyo, Tokyo, Japan.

B9. A Dynamic Analysis of Two-Dimensional Muscle Force Contributions to Lower Lip Movement. J. H. ABBs, Speech Research Laboratories, University of Washington, Seallle, Washington 98195, AND R. NETSELl, Departments of Communicative Disorders and Rehabilitation Medicine, Universily of Wisconsin, Madison, Wisconsin 53706.-Movements of the lower lip in the anterior-posterior and inferior-superior dimensions were recorded along with EMG activity from the orbicularis oris inferior (OOI), depressor labii inferior (DLI), and mentalis (M). Observations of activity in these muscles in relation to simultaneous displacement, velocity, and acceleration of lower lip movement suggest that (1) DLI and OOI act primarily to apply forces in the inferior and superior dimensions, respectively; (2) mentalis is a primary force for protrusion of the lower lip; and (3) the lower lip can be characterized, in a preliminary way, as a simple second-order mechanical system. The implications of the technique and the specific findings will be discussed in relation to development of an analog computer model of the labial-mandibular mechanical system. [Research was supported in part by grant from NIDR.]

B10. Activity of Some Extrinsic and Intrinsic Tongue Muscles in the Articulation of American English Vowels. KATHERINE S. HARRIs" and Lawrence J. RaPHAEL, † Haskins Laboratories, New Haven, Connecticul 06510.-Most previous electromyographic studies of tongue muscles have been limited to those extrinsic muscles which are easily accessible: the genioglossus and palatoglossus. Recent experiments have provided access to the styloglossus, among the extrinsic muscles, and to the intrinsic fibers of the tongue. Thus, a fuller picture of muscular synergisms in the positioning and shaping of the tongue is beginning to appear. This study reports findings from three subjects who spoke 10 American English vowels in a labial consonant frame preceded by schwa. All electromyographic activity was recorded from hooked-wire electrodes inserted into the various muscles by means of hypodermic needles. The results reveal as many differences as similarities between the articulatory strategies of the subjects. It appears that the similar articulatory configurations that underlie the linguistically relevant acoustic structures of the vowels can be achieved by a variety of muscular patterning. [This research was supported by the National Institute of Dental Research.]

- Also at the Graduate Center of the City University of New York.

$\dagger$ Also at Herbert H. Lehman College of the City University of New York.

B11. Tongue and Lip Activity During Australian Aboriginal (Walbiri) Articulation. W. R. Proffit AND R. E. MCGLONE, University of Kentucky, Lexington, Kentucky 40506, AND M. J. Barretr, University of Adelaide, A ustralia.-Recording equipment was transported to Yuendumu, N. T., Australia, to obtain data on tongue and lip activity of aboriginals during speech and swallowing. Strain gauge pressure transducers were fitted into custom constructed plates to be placed in the speaker's mouth to record tongue and lip contact at several locations. Eighteen speakers whose native language is Walbiri acted as subjects. Eight prerecorded Walbiri words and four nonsense words, five repetitions each, were presented to the subjects. These tokens contained three of the Walbiri modifications of the phoneme / $t$ / situated intervocalically in each word. Distinct articulation patterns of both tongue and lips were found for the three phonemes regardless of whether they were located in meaningful or nonsense words. Furthermore, pressure patterns for the phoneme most like the American language $/ t /$ were very similar to patterns for American speakers. This was true even though tongue and lip movements during swallowing were quite different from Americans. [Kenneth Hale assisted in preparing the speech stimulus. Support from the Fulbright program, NIDR, and Southern Society of Orthodontists is gratefully acknowledged.]

B12. A Two-Dimensional Strain Gauge Transducer System for the Lips and Jaw. J. H. ABgs AND B. N. GILBERT, Speech Research Laboratories, University of Washington, Seattle, Washington 98195.-A strain gauge transduction system was designed and constructed for simultaneous observation of the movements of the upper lip, lower lip, and jaw in both inferior-superior and anterior-posterior dimensions. Empirical verification of the system's structural loading factor, frequency response, linearity, and phase response suggests that it provides a favorable alternative to earlier lip and jaw monitoring devices. Some examples of the two-dimensional multistructure movement patterns obtained with this device will be provided and discussed. [Research supported in part by NIDR/grant.]

B13. Intraoral Air Pressure Correlates of Lip Occlusion for Bilabial Stop Consonants. Thomas ShIPp, Speech Research Laboratory, Hospital, San Francisco, California 94121.--The purpose of this procedural study was to determine if a oneto-one correspondence existed between the intraoral pressure curve and complete lip closing and opening for bilabial stop consonants. The faces of two subjects were photographed at high speed (250 fps) in simultaneous anteroposterior and lateral views as they produced a series of words with $/ p /$ and /b/, the midvocalic consonants of interest. A timing signal synchronized the film with the physiologic-acoustic record. 
Results showed that the onset of the intraoral air pressure rise coincided with the moment of complete lip closure while initial lip opening was coincident with the steep fall in intraoral air pressure. These relationships obtained regardless of consonant voicing or stress position, or whether the intraoral catheter was partially obstructed.

B14. Some Relationships between Vocal Effort and Intraoral Air Pressure. R. H. Colton, Experimental Phonetics Laboratory, Department of Otorhinolaryngology, Slale University of New York, Upslate Medical Center, Syracuse, New York, AND W. S. Brown, JR., Communication Sciences Laboratory, University of Florida, Gainesville, Florida 32601.-Previously, Wright and Colton [J. Acoust. Soc. Amer. 51, 141 (1972)] described a power function between vocal effort and SPL that was concave downward with the knee of the function occurring apparently at the subject's most comfortable effort level. A similar function was noted for effort and fundamental frequency. In this study, a similar paradigm was utilized to explore the relationship between effort and intraoral air pressure. Vocal effort for the CVs /da/ and /ta/ was measured by magnitude estimation and production; intraoral air pressure was sensed with a tube-transducer system. Minimum, most comfortable, and maximum effort levels were also determined. The results show that vocal effort is a power function of intraoral air pressure. Furtt_-imore, the function shapes were very similar to those reported by Wright and Colton; the change of slope tended to occur at the most comfortable effort level. Males tended to show the typical function; females tended to display a more straight function. These results tend to suggest the possible existence of two mechanisms involved in varied effort levels, since air pressure rises more rapidly below comfort level than above.

B15. Palatoglossus Function in Normal Speech ProductionElectromyographic Implications. JAMEs LuBker, Dental Research Center, Chapel Hill, North Carolina 27514, JuditH Berezin Salinard, Toms River Schools, Toms River, Nezo Jersey 08753, and Kenneth MaY, School of Dentistry, UNC, Chapel Hill, North Carolina 27514.--Fine-wire-inserted electrodes were placed in the right and left palatoglossus muscles of 14 subjects. Two to four simultaneous placements were accomplished for each subject. Electromyographic signals were recorded while each subject produced a speech sample designed to consider palatal and lingual movements. The data strongly suggest that, while palatoglossus function for the nonspeech act of swallowing is highly consistent within and between speakers, its specific function in speech production is less consistent. In some subjects, the palatoglossus appears to function to actively lower the palate for nasals and at the end of utterances, while for other subjects it does not. In some subjects activity appeared related either to tongue movements or to stretch reflex. Finally, within any one subject, function was often variable. The data are discussed primarily with regard to palatoglossus function, although other aspects, i.e., its variability, which will be discussed in detail in subsequent papers, are also mentioned. [Research supported by NIDR, PHS grants.]

\title{
Session C. Engineering Acoustics I : Acoustic Holography
}

\author{
Gerhard M. Sessler, Chairman \\ Bell Laboratories, Murray Hill, New Jersey 07974
}

\section{Invited Papers (30 minutes)}

C1. A Review of Progress and Applications in Acoustic Microscopy. L. W. Kessler, Research Department, Zenith Radio Corporation, 6001 Hest Dickens Avenue, Chicago, Illinois 60639.-Acoustic microscopy is a relative newcomer to the field of acoustic imaging which will allow the discrimination of micron-size structural detail and will render mechanical microstructure directly observable. Because of the unique characteristics of acoustic wave propagation in materials, potential wide areas of application are anticipated in both the physical and biological sciences. This talk will present a discussion of recent developments in acoustic microscopy techniques and applications. The present efforts to develop experimentally useful systems employ optical methods of sound amplitude detection through various means of coupling. The desired acoustic pattern can be displayed as a hologram or as a conventional image. Further, phase contrast and dark field techniques are also possible. Thus far, resolution better than $10 \mu \mathrm{m}$ has been reported, and it is reasonable to expect that the resolution will soon be improved to the point of equivalence to optical microscopy, viz., about $1 \mu \mathrm{m}$. Comprehensive investigations into possible applications of acoustic microscopy are somewhat restricted at present because of the unavailability of a suitable instrument; however, during the course of preliminary investigations, various interesting and meaningful contrasts have been demonstrated between acoustic and optical micrographs of the same specimens. These will be discussed in the context of their being the first such comparisons to be made rather than in the context of their being definite applications of acoustic microscopy.

C2. Real-Time Ultrasonic Imaging by Bragg Diffraction. G. WADE, Deparlment of Electrical Engineering, University of California, Santa Barbara, California 93106.-Bragg diffraction is one of several principles presently being examined in research to produce optical images of objects irradiated by ultrasound. In this method, laser light is diffracted from the scattered ultrasonic wavefronts as originally predicted by Brillouin in 1921. Historically, some of the first experimental attempts to use ultrasound for imaging were made by Sokolov in his Leningrad laboratory more than 40 years ago. One of the approaches he employed was remarkably similar to the approach presently being pursued 
and suggested in 1966 by Korpel of Zenith. In our laboratory, we have carried out experiments involving sound frequencies of 15,18 , and $25 \mathrm{MHz}$ and more recently $3.6 \mathrm{MHz}$. The images obtained at the higher frequencies are at least comparable to those of competing ultrasonic methods. At the lower frequency, special care must be taken to avoid interference between the image beam and the undiffracted light. This paper will discuss the practical problems encountered and will present theorectical calculations to compare several competing approaches presently being pursued.

C3. Piezoelectric Arrays. R. K. Mueller, Bendix Research Laboratories, Southfield, Michigan 48076.The first practical piezoelectric array was demonstrated by Langevin early this century. It was a composite quartz transmit/receive unit for underwater search and surveillance. A wealth of new piezoelectric materials have since been developed. Sophisticated array processing techniques evolved and became practical with increasing speed and capacity of digital computers. These developments led to the present wide range of piezoelectric arrays with widely differing design objectives. A general survey of the state of the art will be given, and, as a specific example, some design features of a $96 \times 96$ holographic receive array presently under construction will be discussed.

C4. Condenser-Transducer Arrays for Recording Acoustic Holographic Data in Real Time. A. K. Nigam, Bell Laboratories, Murray Hill, New Jersey 07974.- Two-dimensional transducer arrays consisting of a mosaic of condenser-microphone elements appear to be ideal acoustic analogs of the photographic plate for recording holographic data in real time. Such transducer arrays, employing either external or electret biasing, are reviewed in the present paper. In particular, two relatively simple and inexpensive designs [see for example A. K. Nigam, K. J. Taylor, and G. M. Sessler, Acoustical Holography, Volume 4, G. Wade, Ed. (Plenum, New York, 1972), pp. 173-194] are described in detail. Simplicity of design is achieved by using a single solid-dielectric diaphragm, which is shared by a large number of microphone elements of the array, and by employing sequential data sampling, which eliminates expensive repetition of similar signal-processing equipment for each element of the array. Electrical and acoustical characteristics, such as interelement cross talk, element sensitivity, frequency range of operation, and the real-time capability for the two array designs are reported. These are found to compare favorably with the characteristics for the more conventional piezoelectric arrays. Acoustic images made with $16 \times 16$ element prototype arrays of the present design are also discussed.

C5. The History and Current Status of Liquid-Surface Acoustical Holography. Byron B. Brenden, Holosonics, Inc., Richland, Washington 99352.- The interaction of acoustic energy with a free liquid surface provides a means for rapid formation and readout of an acoustical hologram. Although a twostep holographic process is possible, involving recording the liquid-surface hologram on a photographic transparency, the real-time imaging capabilities are of most interest and provide the basis for all of the current use of equipment in industrial testing and biological imaging. The use of acoustic lenses to focus the object field of interest into the hologram is particularly useful for achieving good resolution. Industrial test applications and imaging of biological systems in real time are best illustrated using video tapes or motion pictures.

C6. Summary of Progress and Future Directions in Acoustical Holography. A. F. Metherell, McDonnell Douglas Corporation, Actron Industries, Inc., Monrovia, California 91016.-The author will summarize the papers presented by the preceding invited speakers and review other developments in the field. Current problems and directions for future progress will be discussed.

TUESDAy，28 NovemaER 1972

DOMINION RoOM, 9:00 A.M.

\title{
Session D. Shock and Vibration I : Structural Response and Failure Mechanisms Under Stationary and Nonstationary Random Excitation-Part 1
}

\author{
Harry Himelblau, Chairman
}

Space Division, North American Rockwell Corporation, Downey, California 90241

\section{Invited Papers (25 minutes)}

D1. Statistical Parameters under Stationary Random Excitation. A. G. Piersol, Bolt Beranek and Newman Inc., Canoga Park, California 91303.-Fundamental statistical parameters used to describe stationary random vibrations are viewed with emphasis on those parameters of special concern in structural response and failure prediction problems. Included in the review are correlation and spectral density functions, instantaneous and peak value probability density functions, arbitrary level crossing statistics, and elementary considerations in the first passage problem. The relevance of the various statistical parameters to the prediction of structural fatigue and extreme value failures, 
assuming simple failure mechanism models, is introduced in qualitative terms. Both theoretical and experimental results are displayed to illustrate important relationships. Special attention is given to the importance of underlying assumptions inherent in classical applications, in particular, the excitation normality and system linearity assumptions. Data are presented to demonstrate the impact of common nonlinearities on such response parameters as the instantaneous and peak value probability density functions. Finally, the importance of the stationarity and associated constant parameter system assumptions are discussed from a practical viewpoint. Illustrations are included to clarify the types of errors that result when nonstationary random excitations and responses are analyzed as if they were stationary.

D2. Fatigue and Crack Propagation Analyses under Stationary Random Excitation. H. A. LEYbOLD, NASA Langley Research Center, Hampton, Virginia 23365.-An accurate analytical method for predicting the fatigue life of an aircraft structure under actual operating conditions is not a reality today. Four major parameters (i.e., material behavior, environment, structural configuration, and operational loads) plus the three phases of the fatigue process (i.e., initiation, crack propagation, and fracture) are involved in the prediction process and attest to the complexity of the problem. In addition, each parameter has its own statistical variability, which further complicates the problem. Even when treating each of the variables as independent events, the prediction process is still a complex one. This paper will deal with only one of the variables involved in the overall prediction process, namely, operational or random loads. Many investigators have proposed techniques for predicting life under random loading - with varying degrees of success--based on experiments, empirical rules, and/or past experience. The most noteworthy of those techniques are reviewed, and their capabilities and limitations assessed. The prediction of crack growth in aircraft structures is also of interest from a safety and reliability standpoint, as well as for setting up inspection and maintenance schedules. Current crack-growth prediction techniques, which incorporate fracture mechanics methods, are reviewed and assessed. A bibliography of pertinent references is included.

D3. First Passage Analyses under Stationary Random Excitation. J. T. P. YAO, School of Civil Engineering, Purdue University, Lafayette, Indiana 47907.-A structure fails whenever its response to excitation reaches one or more limiting values. Structural responses include deformations and internal forces, of which the limiting values are based upon various failure criteria for the material. The failure probability of a structure subjected to random excitation can be found as the first passage probability of the response process across the most severe limiting strength values or deformation barriers. This problem can be solved in principle when the structural response is represented by a Markov process. However, exact solutions are available only for some first-order Markov process. Consequently, approximate methods have been suggested by various investigators. In this presentation, an attempt will be made to summarize and review the Poisson approximation with several independence assumptions, two-state Markov process assumption, clump-size estimation, use of an equivalent firstorder Markov process or a renewal process, simulation and numerical diffusion, upper and lower bounds, nonapproaching excursions, and the principle of maximum entropy of the total number of excursions.

D4. Reliability of Structures under Random Loading. RoBert A. Heller Virginia Polytechnic Institute and State University, Blacksburg, Virginia 24061.--Random loads acting on structures can be placed into two broad categories. Into the first category belong loads with large numbers of repetitions such as those produced by wind gusts, ocean waves, noise, boundary layer turbulence, fuel sloshing, or rough roads. Such loads are usually characterized by low-amplitude stationary or quasistationary random processes. Into the second category belong loads whose appearance is less frequent. Loads produced by sonic booms, earthquakes, ballistic impact, severe maneuver loads, hurricanes, tornadoes, and tsunamis are classic examples. These have short durations, of ten large amplitudes, and are governed by nonstationary random processes. While the two groups cannot be completely separated, the first types are usually responsible for the gradual deterioration of structural strength and those in the second class may produce ultimate load failures. Structural response to these random loads depends on basic material properties, as well as on the type of structure, connections, and support conditions. Because structural reliability implies a failure-free operating life, the definition of failure becomes an important parameter. Depending on the structure and its usage, partial failures in conjunction with alternate load paths require consideration. In order to achieve current structural failure goals of the order of $10^{-6}$ to $10^{-\mathbf{a}}$, all the above statistically variable conditions should be considered together. Because of the high complexity involved, however, only individual parts of the problem have been successfully handled in the past. Both theoretical and experimental investigations of the complete problem will hopefully be understood in the near future.

D5. Experimental Fatigue Results under Stationary and Nonstationary Random Vibration. S. R. Swanson, MTS Systems Corporation. Minneapolis, Minnesota 55424.-There is a steadily increasing supply of interesting fatigue test result for materials under the action of random processes, especially in the area of stationary random loading. In the strictest sense, nonstationary random processes in fatigue should be considered quasistationary processes since the fatigue loading is nominally stationary over the long run to failure. It is possible to imagine nonstationary loadings over a complete fatigue life (such as the action of wear or steadily increasing damping in a fatigue test), but actual tests of this nature are not yet available. This paper surveys the field of stationary and quasistationary 
random loading subsequent to the author's 1968 state of the art paper on this topic. One of the most significant findings from this survey is the widespread use of repeated random loading sequences by research groups and working professional committees in interlaboratory test programs. The necessity to analyze service loadings carefully, to separate random and nonrandom aspects, is also dealt with in detail.

Tuesday, 28 November 1972

El Dorado East, 9:00 A.M.

\title{
Session E. Underwater Acoustics I : Propagation I
}

Carey D. Smith, Chairman

Sonar Technology Division, Naval Ship Systems Command, Washington, D. C. 20360

\section{Invited Papers (25 minutes)}

\begin{abstract}
E1. Coherence of Signals, Noise, and Reverberation in the Sea. R. J. URıck, Naval Ordnance Labortory, Silver Spring, Maryland 20910.- Separated hydrophones in the sea show varying degrees of coherence, or waveform similarity, depending on separation, frequency, and kind of sound observed. Over the past 10 years, we have measured the phase coherence of different kinds of signals, noise, and reverberation between vertically separated hydrophones. Such information is basic for the optimum design of arrays. This paper summarizes these measurements. When plotted against normalized separation the coherence as measured by the clipped correlation coefficient is found to lie, as it should, between the value of unity for a plane wave and a curve for isotropic noise; it depends on the vertical angle within which the sound arrives at the two hydrophones. The most coherent sounds observed were signals in convergence zones; the least coherent, the reverberation received from great depths in the deep sea.
\end{abstract}

E2. Project KIWI ONE: Very Low Frequency Acoustic Propagation Measurements in the South Pacific Ocean. D. G. Browing, W. R. Schumacher, and W. H. Thorp. Nere London Laboratory, Naval Underwater Systems Center, New London, Connecticut 06320, AND R. W. Bansistek and R. N. Denham, Defence Scientific Establishment, H. M. N. Z. Dockyard, Auckland 9, Nerv Zealand.-A very low frequency acoustic propagation experiment was conducted along a $10000-\mathrm{km}$ great circle track between New Zealand and the Galapagos Islands. Sound signals set to detonate at the sound channel axis were dropped at $30-\mathrm{km}$ intervals along the entire route by a jet aircraft fying nonstop from Panama to New Zealand. Values of propagation and attenuation loss for the frequency range 10-1000 $\mathrm{H}_{z}$ have been computed from data obtained from listening stations located at both ends of the track.

\section{Contributed Papers (12 minutes)}

E3. Low-Frequency Sound Attenuation in the Indian Ocean. D. G. Browning, E. N. Jones, And W. H. Thorp, Nez London Laboratory, Naval Underwater Systems Center, New London, Connecticut 06320.-With the support of the French Navy, a low-frequency sound propagation experiment was conducted to obtain attenuation coefficients in the Indian Ocean for the frequency range $200-10000 \mathrm{~Hz}$. The experiment was conducted along a $500-\mathrm{km}$ track, which was characterized by a broad sound channel with an axis depth of $250 \mathrm{~m}$. The results are compared with previous measurements in the Red Sea and Atlantic Ocean.

E4. A Study of Very Low-Frequency Attenuation in the Deep Ocean Sound Channel. F. R. DiNa Pol, M. R. Powers, AND D. G. Browning, New London Laboratory, Naval Underwater Systems Center, New London, Connecticut 06320.-The fast field program (FFP) is used to study sound propagation in a deep ocean sound channel for the frequency range 1-200 $\mathrm{Hz}$. Typical sound velocity profiles from the Atlantic and Pacific Oceans are used. Both the velocity profile and bottom depth are assumed to be invariant with range. The channel cutoff frequency is determined and the bottom loss contribu- tion to the measured values of attenuation is estimated. Comparison is made with recent experiments.

E5. Long Range Acoustic Transmission Loss in the Northem Denmark Strait. D. L. Bradley and G. M. Colvin, Naval Ordnance Laboratory, Silver Spring, Maryland 20910.--Long range acoustic transmission loss measurements were made in the northern Denmark Strait area utilizing two aircraft. One aircraft flew prearranged tracks, dropping standard U.S. Navy explosive charges, while the second aircraft remained in the area of deployed calibrated sonobuoys, acting as a receiving platform. Received signals were recorded broad band aboard the monitoring aircraft. These broad-band recordings were analyzed by filtering, squaring, and integrating to obtain energy density in selected $\frac{1}{3}$-oct bands. The Weston values of source level for the explosive charges were used in the calculation of transmission loss. Transmission loss data for several octaves are shown as well as the results of calculations of a loss per unit distance factor [J. D. MacPherson, J. Brit. IRE 26, 293 (1963)]. This factor is obtained by assuming spherical spreading to $1 \mathrm{kyd}$ and cylindrical spreading thereafter and 
calculating the difference between this assumed transmission loss and the measured transmission loss.

E6. Comparison of Under-Ice and Open Water Transmission Loss Measurements in Baffin Bay. T. J. Tulko, Naval Ordnance Laboralory, Silver Spring, Maryland 20910.-An experiment was conducted under early winter conditions to obtain underwater acoustic transmission loss data in ice-free water and water with a broken cover of sea ice under similar refractive conditions. Measurements of sound transmission and ambient noise were obtained using modified calibrated sonobuoys and standard U. S. Navy explosive sound sources. The received acoustic signals were recorded broad band and later analyzed in selected 3 -oct bands. Transmission loss data are presented with the results for ice-covered water compared with the results for ice-free water. Acoustic transmission loss in Baffin Bay under refreezing conditıons is higher in ice-covered water than in open water.

\section{E7. Abstract withdrawn.}

E8. Acoustic Flux Density for Ray Propagation in Discrete Index Media and in Gradient Index Media. D. G. BurkHARD AND D. L. Shealy, Department of Physics and Astronomy, University of Georgia, Athens, Georgia 30601.-General formulas are derived for the energy per unit area per unit time (fux density) over an arbitrary receiver surface for acoustic ray propagation in discrete index media and in gradient index media. The equation of the deflected ray coupled with the equation for the deflecting surface and the equation for the receiver surface provide a transformation which maps an element of deflecting area onto the receiver surface, thereby enabling one to calculate the flux density over the receiving surface. A formula for the flux density along a ray path is obtained as a special case. An equation for the caustic surface is obtained from the latter. As an example, fux density contours are calculated over a planar surface for plane-wave acoustic radiation reflected from a sphere embedded in a gradient index medium.

E9. Statistical Averages as Estimates for Acoustic Intensity. L. Bruce Palmer, Naval Research Laboratory, Washington, $D$. C. 20390.-Basic to the study of undersea sound propagation is the prediction of acoustic intensity at some fixed receiving point. In ray acoustics, the total intensity at a point is taken to be the sum of the individual intensities of the single ray, or ray-bundle, arrivals at that point. At the heart of the ray acoustics approach is the estimation of the geometric spreading loss suffered by a single arrival. The usual formulations of spreading loss require the evaluation of a derivative of either depth or range with respect to initial ray angle. The calculation of this derivative has proven to be tronblesome in practice, especially for long ranges from the sound source. By the representation of a single ray intensity at a point as a statistical average of the intensities of "neighboring" rays, the estimation of the derivative is avoided and smoothed intensity estimates are obtained that are particularly applicable at long ranges from the source. The statistical average can be formulated to be performed over either depth or range. In addition, these formulations can be applied in such a manner as to provide án adjustment to intensities near caustics.

Tuesday, 28 November 1972

El Dorado West, 9:00 A.M.

\author{
Session F. Architectural Acoustics I : Room Acoustics
}

Harold R. Mull, Chairman

80 Danbury Road, Wilton, Connecticut 06897

Contributed Papers (12 minutes)

\title{
F1. Abstract withdrawn.
}


F2. The Design of a 360-Degree Symphonic Facility. ChristoPHER JAFFe, Christopher Jaffe, Inc., Norwalk, Conneclicul 06852. - In 1971 the first $360^{\circ}$ concert facility for a major symphony orchestra was completed at Ontario Place, Canada, as a summer home for the Toronto Symphony. This paper describes the physical and electroacoustic criteria developed to meet the program requirements, discusses the degree to which the criteria fulfills design objectives, and the response of artists and audiences to this intimate, less formal performance environment.

F3. Acoustics for Opera: Our Experience at Indiana University's New Musical Arts Center. R. LAWRENCE KIRKEGAARD, Bolt Beranek and Newman Inc., Downers Grove, Illinois 60515, and Robert B. Newman and Theodore J. Schultz, Ball Beranek and Newman Inc., Cambridge, Massachusetts 02138.The new 1460-seat Opera Hall at Indiana University has been widely acclaimed for its architecture, its advanced theater technology, and its acoustics for opera and concert. This paper describes the physical characteristics of the hall in general and its acoustical characteristics in particular. Both objective data and subjective evaluations ol its opera and concert uses are presented and compared with design criteria and functional objectives.

F4. On the Loudness of Music Rooms. Rein PIRn, Bolt Beranek and Newman Inc., Downers Grove, Illinois 60515.Free-field measurements of instrumental music suggest close correlation between the $A$-scale londness of music and the mid-frequency response of rooms in which it is played. Other measurements, utilizing a steady-state random noise source, establish a typically increasing order of loudness for spaces ranging from concert halls to practice rooms. While loudness consistently increases with decreasing absorption and cubage, often the number of musicians using the room does not correspondingly decrease. It is shown that rehearsal and ensemble rooms, in particular, are louder than preferred by most musicians and that reduced reverberance is accepted in preference to excessive loudness. Alternative solutions, with room volume and reverberation time as parameters, are discussed.

F5. Practical Determination of the Linear System Impulse Response of a Room. Norman C. Martin, Deparlment of Mechanical Engineering, University of Houston, Houston, Texas 77004.-A review is made of the possible methods to obtain the impulse response of a room. Included in this review are shock stimulus, swept frequency analysis, and correlation technique using a white noise input. The latter technique is readily adaptable to computer processing (either hard wired or soft wired). The difficulties associated with each method are put forward as are the advantages. Emphasis is placed on the correlation technique because of the advantages that can be gained from further processing of the gathered data. It is pointed out that, with the correlation technique used, although the effects of the system up to the source input (say a speaker) are totally removed, the speaker modification to the actual room impulse response is a difficult quantity to deal with. Because of the inherent rise time in any impulse response, the speaker impulse response is not minimum delay (or minimum phase) and hence an exact convolution inverse operator is not stable. Methods to deal with this are discussed and practical results from a Wiener filter approach are presented. [Supported in part by two grants from NASA.]

F6. Design Practice Relating to Acoustics in Schools. William K. Connor, TRACOR, Inc., Austin, Texas 78721, AND Roger Benasutti, Conwed Corporation, St. Paul, Minnesota 55101.A questionnaire survey of 401 architectural firms, completed in 1968, was designed to elicit information regarding usage of acoustical and other materials in schools, reasons for choice of materials, general design practice, and acoustical problems encountered. Of schools recently designed, $73 \%$ were of closed plan type and $27 \%$ of open plan type. Absorptive classroom ceilings, primarily of the acoustical board or tile type, were used in $\mathbf{9 4 \%}$ of all schools. These were chosen for acoustical reasons, but also for economy and as part of integral ceiling systems. Carpeting was more prevalent in open-plan schools and was chosen for both acoustical and aesthetic reasons. Over half the schools were reported to have acoustical problems, predominant among which was sound transmission between class areas. This problem was associated with use of the open plan and also with inadequate wall and ceiling constructions. [Work supported by the Acoustical and Insulating Materials Association.]

F7. Modeling Sinall Room Responses with a Real-Time Digital Filter. T. H. Curtis, D. A. Berkley, and T. B. Bateman, Bell Telephone Laboratories, Incorporated, Holmdel, New Jersey 07733.-A real-time digital filter system designed for speech processing [D. A. Berkley and T. B. Batemen, Int. Congr. Acoust., Budapest (1971)] has been used to model the response of several small rooms. Finite impulse-response filters corresponding to room transfer-function magnitude and phase were synthesized using (1) frequency sampling [L. R. Rabiner and R. W. Schafer, IEEE Trans. Audio Electroacoust. 19, 200 (1971)] realized with parallel second-order filter sections and (2) time domain design implemented in direct form. Both allow approximately $20 \mathrm{msec}$ of the response in a $3-\mathrm{kHz}$ bandwidth to be modeled in real time. In principle, any response can be modeled using this system by overlap processing (non-real time). Using the real-time filter, we have studied the effects of modifying the spectral and temporal properties of the room impulse response (see Berkley et al., Paper F8). Both design methods work but noise considerations rapidly become important when using frequency sampling. [Some aspects of this paper were orally presented at the 1972 International Conference on Communications, Philadelphia.]

F8. Effects on Speech Perception of Modifying the Impulse Response of a Small Room. D. A. Berkley, T. H. Curtis, ANd J. B. Allen, Bell Telephone Laboratories, Incorporated, Holmdel, New Jersey 07733.-A linear model of the ear as a parallel bank of filters and the image solution for room response are outlined. These models are used to predict the perception of speech under certain controlled variations in the impulse response of a small room. Using either a hardware digital filter or a computer simulation, room impulse responses have been measured, modified, and their effect on speech examined. The investigations included linearizing the phase of and truncating the impulse response, examining an all-pass filter with a real room phase characteristic and observing the effect of the time complement of a truncated impulse response

F9. Miniature Anechoic Room Design. W. M VIEgrock* AND M. J. Crocker, Ray W. Herrick Laboratories, School of Mechanical Engineering, Purdue University, West Lafayette, Indiana 47907.-The design of a small portable anechoic room is discussed. The room was constructed with double wall vibration-isolated panels. The wall transmission loss was predicted using London's theory. 'The room suspension system was designed using a lumped-mass model for isolation from floor vibrations. The design of the room utilized 8-in.-long polyurethane foam wedges. Using an impedance tube, mea- 
surements predicted a lower cutoff frequency of $270 \mathrm{~Hz}$ for the design of wedge chosen. Inverse square law measurements and noise reduction measurements of the completed miniature anechoic room are given. [This work was supported by Westclox Division, General Time Corporation, LaSalle, lllinois.]

* Present address: Lockheed Missile \& Space Co., Sunnyvale, Calif.

F10. Probability Density Function of the Time Average of Sound Pressure Level Measurements in a Reverberant Room Excited by Random Noise. Douglas J. Ingalls, Carlyle Compressor Company, Syracuse, New York 13201.-The probability density function of the time average of the sound pressure levels (measured in decibels) of a reverberant room excited by a finite bandwidth of random noise is (approximately) Gaussian for a stationary source and stationary receiver. The mean of the distribution for a finite integration time is less than the mean for an infinite integration time. The difference (in decibels) is given by $4.8 / B T$, where $B$ is the bandwidth in hertz and $T$ the integration time in seconds. The normalized variance of the distribution is given by $19 / B T$. The results imply that narrow-band measurements in a reverberant room need to be carefully defined if errors in the measurement due to finite integration times are to be minimized.
F11. New Reverberant Room Acoustic Test Facility. Curtis I. Holmer and Douglas W. Andersen, Bolt Beranek and Newman Inc., 50 Moulton Street, Cambridge, Massachusetts 02138. - A test facility recently constructed at $\mathrm{BBN}$ consisting of a pair of reverberant rooms for transmission loss (TL) measurements and sound power level measurements is described. Because of spacc limitations, existing facilities, and the need to measure models to high frequencies, the rooms were limited to $3100 \mathrm{ft}^{3}$ volume. The principle instrumentation used for measurements includes an electrically scanned multiple microphone array and a real-time analyzer. $A$ unique feature of the facility for TL measurements is a modular demountable steelfller wall which permits easy alteration to accommodate samples from $3 \mathrm{ft} \times 3 \mathrm{ft}$ to $9 \mathrm{ft} \times 14 \mathrm{ft}$ in size. A major concern during the evaluation of the facility was the intention to make sound power level measurements in accordance with ANSI S1.21. It was found that by using an eight microphone array and a rotating diffuser the rooms easily meet the requirements for measuring broad-band noise. For measuring tones, however, a low-frequency absorber and two suitably chosen source positions were also required in order to meet the performance requirements of the standard. Criteria for the selection of source positions and rotating diffuser operating conditions are discussed.

\title{
Session G1. Community Noise-State of the Art
}

\author{
R. R. Audette, Chairman \\ Burgess Industries, 8108 Carpenter Freeway, P.O. Box 47146, Dallas, Texas 75247
}

\section{Invited Papers (times as indicated)}

G1-1. Introduction by Chairman. R. R. Audette, Burgess Industries, Dallas, Texas 75247.-A brief introduction starting the purpose of this session, whom we are trying to reach, and how we plan to do this.

G1-2. Noise Control by Regulation. Elzzabeth Cuadra, Office of Noise Abalement and Contral, Environmental Protection Agency, Washington, D. C. 20460.-An overview is presented of the current and anticipated future roles of the Environmental Protection Agency in what is hoped will become a cooperative, nationwide noise abatement program. As background, a brief review is given of (a) the estimated current extent and severity of the "noise problem" outside the work place (based on work performed as input to EPA's "Report to the President and Congress on Noise"); (b) major existing federal statutes or regulations relating to noise abatement; and (c) the current general status of state and local government noise abatement programs. The EPA's anticipated future role (hased on legislation pending before the Congress at the date of this abstract) is outlined, and the desirability of continued and increased noise abatement activities, especially at state and local levels, and also within other federal agencies, is emphasized. Clarifying examples are presented of the methods by which state and local governments can (and should) control environmental noise, even when sources of noise are involved for which preemptive federal noise emission regulations will have been promulgated under the (pending) Noise Control Act of 1972.

$$
\text { 9:30 }
$$

G1-3. Transportation Noise-Airplane Noise Reduction. Harvey H. Hubbard, NASA, Hampton, Virginia 23365. - Current Federal noise regulations will be discussed along with their impact on community noise exposure. Brief references will be made to specific technical areas where significant advances in noise reduction have been made and to noise reduction goals for the future. 
G1-4. Community Noise-Surface Transportation. G. J. THiessen, National Research Council, Ottawa, Ontario, K1A OS1, Canada. - Noise due to surface transportation is still rising, but there is evidence that it is leveling off. The important change in the last five years has been the increase in knowledge of the relative importance of the noises from exhaust, intake, engine radiation, tires, and airflow and the extent to which they can be modified at reasonable cost. Trucks and motorcycles are still the most intrusive noise sources, with the latter showing some signs of improvement with the introduction of a water-cooled model by Suzuki. Radically new engines, such as the Wankel and gas turbine engines, are still too close to the experimental stage to show what they can do to decrease noise and abide by emission standards. Legislative control is the primary mechanism for overcoming the competition problems, which make manufacturers reluctant to incur the cost of noise reduction in the worst noise sources. More inclination to experiment has been shown by public bodies in road layout, design, and construction of barriers, but not always with full knowledge of the pitfalls and limitations of this approach.

10:00

G1-5. Noise of Homes. John R. Schreiner, Carrier Air Conditioning Company, Syracuse, New York 13201. - Our homes are filled with products that provide comfort, convenience, and entertainment for the occupants and coincidentally produce noise. Most of these products operate only for short periods of time when users turn them on, but some cycle on and off continuously in response to automatic controls. Most are normally operated inside homes but sorne of the noisier ones are used outdoors and thereby contribute to community noise. Sound outputs vary widely between different types of products and to a lesser extent among different models of the same type, and many also vary with the mode or cycle of operation. The effects they produce also vary with the relative locations of the people exposed and their occupations at the time. While these factors add complications, considerable progress has been made on sound rating standards for many of these products, notably in the air conditioner industry, and quieter products will typically result from sound rating programs.

$$
10: 30
$$

G1-6. Community Noise. C. R. Bragdon, Department of City Planning, Georgia Institute of Technology, Allanla, Georgia 30332. - The magnitude of the existing urban noise problem is presented in terms of its acoustical characteristics and environmental impact. The current status of community noise research, including acoustical as well as social analysis, is summarized. Implicated environmental health effects involving potential hazardous and nuisance responses to community noise are identified. Public as well as political recognition of community noise is compared to other environmental problems. Existing and proposed noise management programs at all governmental levels are assessed in terms of their relative effectiveness, including protecting the public's interest. Presented are examples of a few existing programs that sufficiently address the problem. The current inadequacies and requirements for more active and comprehensive noise managernent programs are apparent. Effective coordination of environmental program at all governmental levels is also needed. Present governmental policies and their ability to control community noise successfully are discussed, including considerations in establishing standards.

\section{1:00}

G1-7. Industrial Noise in the Community-Current Views. Lewis S. Goodfriend, Morristown, New Jersey 07960.- Noise from industrial plants has continued to be a problem for both the plant managements and their neighbors. Several recent studies have indicated that the majority of persons in residential areas are exposed to ambients set by transportation noise sources. This is of little comfort to the immediate neighbors of an industrial plant whose noise dominates a small region surrounding the plant. Recent acceptance of the $A$-weighted sound level to rate noise, results of several Federal studies, and an evaluation of the Normalized Community Noise Equivalent Level (NCNEL) have changed the community-industry situation little, but prepare the way to develop workable methods for rating noise situations in a manner similar to the earlier Composite Noise Rating, but without measurement of the spectrum. Furthermore, the interrelationship of a community and its industrial neighbors appears to play a major part in the acceptance of the noise levels surrounding the plant. This leads to the conclusion that there is no universal method of rating community noise that will satisfy any given percentage of neighbors in a community without first determining attitudinal factors that can be applied in the noise evaluation as weighing factors. Further research is currently under way. This effort is critical since current work to reduce transportation noise may lead to a situation where industrial noise becomes dominant in the major portion of many more communities.

\section{$11: 30$}

G1-8. The Citizen Response to Noise Since 1967. Robert Alex Baron, Executive Vice President, Citizens for a Quieter Cily, Inc., P. O. Box 7777, Ansonia Stalion, New York, New York 10023.-Since 1967, the citizen is on the offensive. He has learned how to intensify awareness of noise pollution. His educational techniques have become more sophisticated. Pressure points have been discovered and are being applied. Environmental noise is now being recognized by some acousticians and polluters. However, significant moves toward abatement have yet to be taken. While the hearing loss 


\title{
84 TH MEETING - ACOUSTICAL SOCIETY OF AMERICA
}

aspect of occupational noise is now being recognized in legislation, the nonauditory aspects are totally neglected. The citizen is, therefore, now including a concern for occupational noise while intensifying his battle against environmental noise exposure. The noise receiver is still asking the acoustician to move from measurement for the protection of vested interests to abatement for the sake of human interests.

Tuesday, 28 November 1972

El Dorado East, 2:00 P.M.

Session G2. Community and Vehicle Noise

\author{
T. E. Sindon, Chairman
}

Department of Mechanical Engineering, University of British Columbia,

Vancouver 8, B.C., Canada

\section{Contributed Papers (12 minutes)}

\begin{abstract}
G2-1. Hazardous Jet Noise Radiation at Schools near Los Angeles International Airport. W. C. Meecham and S. R. LANE, School of Engineering and Applied Science, University of California, Los Angeles, California 90024.-In the first part of a two part study, indoor and outdoor measurements of jet aircraft noise were obtained at seven schools beneath the eastern approach paths to Los Angeles International Airport. At five elementary schools, the jet noise in the schoolyards ranged from 100 to $120 \mathrm{dBC}$ and constituted a high risk of hearing damage for the young children. The frequency of jet aircraft overflights averages 1 per 2 min for a total of over 200 per school day (8:30 A.M.-2:30 P.M.). The corresponding jet noise levels in the classrooms, $80 \%$ of which are not air conditioned or acoustically treated, ranged from 80 to $96 \mathrm{~dB} A$, and caused total disruption of the communication and learning processes. An investigation of aircraft operation alternatives indicated that the noise could be reduced by $10 \mathrm{~dB}$ or more if engine power were reduced when the aircraft approached the school areas, or if the aircraft approached at higher altitudes and landed further to the west on the unused 6000 -ft-long portion of the $12000-f t-l o n g$ runways.
\end{abstract}

G2-2. Computer Generated Airport Noise Contours. J. A. Spencer and T. H. MeYer, Bolt Beranek and Newman Inc., Cambridge, Massachusetts 02138.-Existing airport noise prediction programs are incapable of dealing with complex flight paths and normal dispersion of light paths due to variations in aircraft gross weight or pilot technique. A computer program that is capable of handling these problems has been developed, using an air traffic control simulation program as its base. The original air traffic control program is capable of very accurate simulation of aircraft motion in an airspace, including random deviations from assigned parameters and automatic metering of traffic using holds, delaying turns, etc. A grid of noise measuring points, of arbitrary extent, is superimposed on the airspace and constant recording of aircraft noise at each location is simulated. At the end of a "run," a contour plot of noise levels in the grid area is produced. Variations in traffic density, controller technique, traffic patterns, and runway use can be simulated, and the results of such variations conpared directly.

G2-3. Noise Intrusion from Police Helicopters over Los Angeles Communities. W. C. Meecham and S. R. LANE, School of Engineering and Applied Science, University of California, Los Angeles, California 90024.-Measurements of police helicopter noise were made during surveillance fights over communities in several cities in the Los Angeles area. Small light observation-type helicopters (Hughes 269 and 300 and Bell 206) are used for emergency and surveillance work by the police departments of most cities in the Los Angeles area, and by the sheriff's departments in the southern California seven county area. These helicopters typically fy over the various communities several times each day and during the late evening hours. Operation of these helicopters at 500- to 1000-ft altitudes results in noise levels of 70-80 dB $A$ in the residential communities. Helicopter flights over schools and colleges caused noise levels of $65-75 \mathrm{~dB} A$ inside the classrooms. This is $10-20 \mathrm{~dB}$ above the maximum allowable limit of $55 \mathrm{dBA}$ for acceptable speech communication in offices and classrooms. In the Santa Monica area, late night flights and search patterns caused noise levels of 100-115 EPNdB over hospitals and convalescent homes. These levels are in violation of municipal noise codes and exceed the federal landing noise limits for new jet aircraft.

G2-4. Exposure of Communities to Low-Frequency Noise from Aircrafts and Rockets. D. Kiro, U. FEHR, M. BURNSTEIN, B. Iron, and J. Goldman, Mamash Applied Science Laboratories, Ness-Ziona, Israel.--Data collections, in the low-frequency noise region (below $100 \mathrm{cps}$ ), in communities located near airports were analyzed for the purpose of volumetric coverage. The following factors, such as residential areas under influence, volume of noise, time of noise, and range of influence, have indicated the importance of these studies. Isosurfaces of SPL of jet planes at altitudes of up to $7000 \mathrm{ft}$ are shells in the shape of pears, with the top directed towards the ground. The top covers a minimum area of $12000 \mathrm{~km}^{2}$. The pearshaped envelope is pulled in the direction of the flight, and this produces a time delay and altitude dependence between the community exposure to radiated noise, and the instantaneous location of the plane. Typical time exposures for altitudes of 1000 to $7000 \mathrm{ft}$ are $45-74 \mathrm{sec}$. This low frequency does not raise complaints because of the human ear's limitations; nevertheless they might be harmful. Furthermore, infrasonic frequencies ( 4 cps and below), having energies of $100 \mathrm{~dB}$ and more, cover $10000 \mathrm{~km}^{2}$ and have durations of up to $30 \mathrm{~min}$. Studies of physiological effects on animals and humans are of importance in this field. If these frequencies are harmful or bothersome, the jet engines should be designed to dissipate these energies at higher frequencies which attenuate strongly and do not propagate long distances in the atmosphere. 
G2-5. Theoretical Interpretation of Recent Experiments of Helicopter Noise Propagation in Urban Areas. Allan D. Pierce and Wayne A. Kinney, Acoustics and Vibration Laboratory, Massachusetts Institute of Technology, Cambridge, Massachusetts 02139. - In a recent series of experiments jointly conducted by the DOT/TSC, the Coast Guard, and MIT, recordings were made while a helicopter flew over (1) an array of microphones placed in an open field and (2) a similar array placed in the center of a street flanked by tall buildings. The differences between the two sets of data (initially lower, then higher levels for the urban data) is similar to that predicted previously from laboratory scale model experiments by Lyon, Pande, and Kinney [Summary Rep. DOT-TSC-93]. In the present paper, a somewhat detailed, theoretical explanation is attempted based on a geometrical acoustical model modified to include edge diffraction. A general expression is first derived for the effective gain due to building presence when sound is excited by a monochromatic point source. This is then used to find the mean and variance of octave-band filtered broadband noise for random listener location near the buildings and near the center of the street. The data is then discussed in terms of these theoretical predictions.

G2-6. The Effect of Sample Size on Freeway Noise Measurements. J. Stephen O'Neall and C. E. Wallace, School of Engineering, Arizona State University, Tempe, Arizona 85281. - Traffic noise levels were recorded along two freeways having significantly different traffic densities. These recordings were analyzed to yield the $A$-weighted noise levels $L_{50} \%, L_{10 \%}$, and $L_{1 \%}$ for a range of sample sizes. Standard deviations of these noise levels were computed and plotted against both the duration of the sample and the number of vehicles passing within the sample. The results show that the standard deviations of the measurements vary with traffic density when plotted versus sample duration but do not depend on traffic density when plotted against the number of vehicles in the sample. For the measurement of $L_{50 \%}, L_{10 \%}$, and $L_{1 \%}$, a $1-\mathrm{dB} A$ standard deviation requires 100,160 , and 300 vehicles, respectively. Similarly a $3-\mathrm{dB} A$ standard deviation requires 19,25 , and 90 vehicles, respectively.

G2-7. Investigation of the Aerodynamic Noise Generating Region of a General Electric T-58 Jet Engine by Means of the Simple Source/Fluid Dilatation Model. Patrick M. HurdLE and W. C. Meecham, University of California, Los Angeles, California 90024, And Brent Hodder, NA.SA A mes Research Center, Moffett Field, California 94035.-Experimental tests were carried out at NASA Ames Research Center. Crosscorrelation measurements were made between the static pressure fluctuations, as measured with a $\mathrm{B} \& \mathrm{~K} \frac{1}{4}$-in. microphone fitted with a nose cone, and the lar-field radiated sound. These measurements were made for two different probe positions and a large number of far-field positions at various angles. In addition, each test geometry was run for jet exit velocities at four different Mach numbers. The measured, normalized cross-correlation functions varied between 0.155 and 0.004 . These values depended upon the angular position of the far-field microphone, the jet exit velocity Mach number, and the position of the probe. In addition, the cross-correlation technique was employed to study the symmetry of the farfield radiated sound. Third-octave analysis of both the probe signal and the far-field radiated sound were made. The results of these measurements will be reported.

G2-8. Consulting Experience with the New Federal Highway Noise Standards. Lawrence G. Copley, Cavanaugh Copley Associates, Newton, Massachusetts 02158.-In April 1972, the Federal Highway Administration issued its interim noise standards [Policy and Procedure Memorandum 90-2: Interim Noise Standards and Procedures for Implementing
Section 109(i) of Title 23 U.S.C.]. These standards will apply on an interim basis to all Federal Aid highway projects submitted for location approval after 1 July 1972. This paper addresses procedural aspects of the Standards rather than policy questions. The paper draws on experience in performing noise evaluation studies in accordance with the Standards for several typical highways projects. Three key features of the Standards are discussed. First, $\mathbf{L 1 0}$ as a measure of community noise level at different times of the day is reviewed in comparison with L.50, which is easier to forecast accurately and less expensive to measure in the field. Second, the use of maximum traffic volume noise levels is reviewed, given that highway noise may be more annoying in off-peak hours such as evening or nighttime, when traffic volumes are less and the highway noise generally has a much different character. Finally, the highway noise Iorecast procedures prescribed in the Standards are discussed.

G2-9. Vehicle Noise Measurements and Community Noise Standards. Anita Lawrence, School of Architecture, University of New South Wales, Kensington, 2033, Australia.Although measurements of road vehicle noise emission made according to ISO or SAE or similar methods have the asset of consistency, their uselulness in predicting the noise annoyance caused by such vehicles is limited, particularly in the case of large commercial vehicles. For nonmotorway traffic in built-up areas, the stop-start condition is most common. Field measurements indicate that acceleration from a stationary start in the lowest forward road gear gives useful results. In addition, body noise may be measured by driving the vehicle at constant speed over a rubber "bump" strip. Vehicle noise limits should be related to community noise standards; however, the subjective equivalence between the annoyance caused by a continuous stationary noise source and that caused by a traffic stream is not easy to establish.

G2-10. A Comparison of the Predicted and Actual "Noise Pollution Level" for Vehicular Traffic on Limited Access Highways. M. J. Oslac, The Noise Control Laboratory, The Pennsylvania State University, University Park, Pennsylvania 16802.-A superhighway near a suburban community formerly connoted convenience and instant accessibility to job, recreation, shopping, etc. Today, land values are more often relating to environmental serenity. This awareness of highway noise has prompted Environmental Impact Studies prior to the final design of new highways. The surrounding environment must be protected at least to the extent of the recently adopted FHWA Policy and Procedure Memorandum 90-2, which for a 24-hour period sets the noise limits which may not be exceeded more than $10 \%$ of the time. While this may be a good starting point, the Policy lacks the requirement that a measure of the background be made. Robinson's Noise Pollution Level (NPL) provides a more realistic value of annoyance Irom vehicular traffic in that it provides for inclusion of the background. It thus offers a possibly better guideline. The recent study described in this paper utilized the $L_{10}$ and $L_{90}$ values calculated from the NCHRP Report No. 117 to predict the NPL for four eastern United States communities adjacent to limited access highways. Measurements were made at the four sites and an exact NPL was calculated using the energy mean value and the standard deviation. An attempt was then made to correlate these values with community response questionnaires. These values were also compared to the $L_{10}$ levels in PPM 90-2. The implications of using NPL in Environmental Impact Studies is discussed.

G2-11. Noise Generation Mechanisms for Passenger Car Tires. T. E. SIDDON, Department of Mechanical Engineering, University of British Columbia, Vancouver, B. C., Canada.Starting with the premise that intense aerodynamic mech- 
nisms are responsible for a significant fraction of the noise radiated by a "relatively smooth" tire rollling on a "relatively smooth" surface, experiments have been conducted to identify and quantify such mechanisms. The experiments, conducted on a passenger car vehicle equipped with rib-type tires, include flow visualization studies and fluctuating pressure measurements both in the vicinity of the tire-road interface and in the acoustic far field (i.e., with the microphone mounted at the end of a 5-ft boom). A unique technique, involving cross correlation between the near-field pressure and the far-field sound measured by the boom mounted microphone, yielded significant information on the distribution of acoustic sources in the vicinity of the tire. These measurements indicate that a strong unsteady flow field does indeed exist in the region immediately behind the tire-road interface, and that this unsteadiness makes a dominant contribution to the noise. Alternative models for the aerodynamic mechanism, based on Hayden's "air-pumping" hypothesis, and a newly advanced "vortex interaction" process, are described and compared.

G2-12. A Low Cost Approach to Area-Wide Community Noise Monitoring. T. E. Siddon and K. D. Harford, Acoustical Engineering, Suile 3, 690 Number Three Road, Richmond, British Columbia, Canada.-A new approach to noise survey work emphasizes the importance of spatial and temporal noise level variations with less stress on frequency content. It is shown that a low cost sound monitoring package, $A$ weighted at the input stage, and with a dynamic range of
$45 \mathrm{~dB}$, yields adequate information on which to base a concise, statistical model of the acoustic landscape. Because of the low cost of individual terminal units, a large number of units may be operated over an extended land use area. This ensures that important spatial information is not lost. Such a detailed picture is not possible with the more conventional sequential (point to point) techniques that are normally employed. The format for a typical area-wide noise level survey is presented, and the usefulness of the data obtained to urban planners and legislators is briefly described.

G2-13. In-Service Tire Vibration Investigation. WilliaM F. Reiter, Leon J. Harper, and Allen C. Eberhardt, Center for Acoustical Studies, P. O. Box 5801, North Carolina State University, Raleigh, North Carolina 27607.-An experimental research program has been initiated to measure and correlate the sound and vibration spectra of in-service truck tires. The objective of this work is to obtain a detailed bank of tire sound and vibration data that will aid in a better understanding of tire noise generating mechanisms and how they are influenced by important parameters such as load, tread type, tread wear, and speed. This work extends the recent work completed by the National Bureau of Standards entitled "Peak $\boldsymbol{A}$-weighted Sound Levels Due to Truck Noise." Initial results of the telemetered vibration data for typical truck tires will be presented. [The Office of Noise Abatement, U. S. Department of Transportation, is acknowledged for support of this próject.]

TUESDAY, 28 NOVEMBER 1972

El Dorado West, 2:00 P.M.

\title{
Session H. Physical Acoustics I : Ultrasonics and Liquid Crystals
}

\author{
BILl D. Cook, Chairman \\ Cullen College of Engineering, University of Houston, Houston, Texas 77004 \\ Invited Papers (30 minutes)
}

\begin{abstract}
H1. Properties of Liquid Crystals. Glenn H. Brown, Liquid Crystal Inslitute, Kent State University, Kent, Ohio 44242.- - Molecular geometry is important in interpreting the properties of liquid crystals. In addition, the molecular packing in different liquid crystalline structures will be presented and correlated with the properties of the liquid crystalline state of matter. This information on molecular packing will be focused essentially on thermotropic liquid crystals, but some information will be presented on lyotropic ones. A brief synopsis of some of the most interesting applications of liquid crystals will be presented.
\end{abstract}

H2. Visualization of Ultrasonic Waves Using Liquid Crystals. P. Greguss, Department of Ophthalmology, New York Medical College, New York, New York 10029.- The search for a device that transfers acoustic wave information into equivalent light information began its rebirth after the first ultrasonic holograms were recorded on sonosensitive plates in 1964/1965. About the same time, the heat sensitivity of liquid crystals which shows up in color was introduced to NDT to find flaws. Since when an ultrasonic image is projected onto a suitable absorbing material it is converted into a corresponding spatial temperature variation over the absorber, it is clear that by coating the absorber with a layer of liquid crystals the intensity distribution of the ultrasonic field will be visualized. Examples of this technique will be presented. In nematic liquid crystals, five electrooptic effects-dynamic scattering, voltage-controlled optical activity, fast turnoff mode, deformation of vertically aligned phases, and guest-host interactions-can be observed, all of which may be affected by ultrasonic waves. Therefore, they can be used to transfer acoustical wave information into light information. The birefringent properties of a new specially prepared liquid crystal cell (AOCC) offer a new real time acoustical-tooptical display, which can also be used to record ultrasonic holograms. The pluses and minuses of the AOCC will be discussed in connection with a short movie.

H3. Liquid Crystals and Broken Symmetry Hydrodynamics. Tom C. Lubensky, Department of Physics, Universily of Pennsylvania, Philadelphia, Pennsylvania 19104.--A general approach to the determination of the equations governing linearized hydrodynamics in systems with broken con- 
tinuous symmetries is introduced and applied in detail to nematic liquid crystals. The hydrodynamical mode structure of nematics is obtained from these equations. Application of the above techniques to the determination of the hydrodynamical equations for cholesterics and smectic liquid crystals is briefly discussed. The complete mode structure for these systems is then reviewed. [Supported in part by grants from the National Science Foundation and the U. S. Office of Naval Research.]

H4. Ultrasonic Measurements in Liquid Crystals. S. V. LeTCHER, Depariment of Physics, Universily of Rhode Island, Kingston, Rhode Island 02881.- Although ultrasonic measurements in liquid crystals were first made more than 15 years ago, only in the last few years has there been a dramatic increase in interest and in the rate of progress. Both longitudinal transmission and shear reflectance measurements have now been performed in smectic, cholesteric, and nematic liquid crystals. The following topics will be among those discussed: temperature and frequency dependence of absorption and velocity, absorption at nematic-isotropic phase transitions, and anisotropic propagation in oriented nematics.

\title{
Contributed Papers (15 minutes)
}

H5. Use of Encapsulated Liquid Crystals to Demonstrate Temperature Increases due to Large Stress Amplitudes in Piezoceramic Bodies. Paul M. KEndrg, Ordnance Research Laboratory, The Pennsylvania Slate University, University Park, Pennslyuania 16802.-When unloaded piezoceramic bodies are driven at high stress amplitudes at their resonant frequencies, considerable heat is produced, with the maximum heating occurring at the nodes (regions of maximum stress). The resulting temperature increase is easily demonstrated by placing on or cementing to the vibrating bodies, films of encapsulated liquid crystals (ELC). The ELC is protected on the viewing side with a sheet of Mylar and covered with black ink on the other. Most suitable films are those with a relatively small temperature range for the visible spectrum. When the vibrating body is driven with large stess amplitudes, a bright colored spot (red end of spectrum) appears in the region of the node. The color rapidly moves through the spectrum to blue and the area of the colored region expands. Implications of this technique are discussed.

TUESDAY, 28 NOVEMRER 1972

EMPIRE RoOM, 2:00 P.M.

\section{Session I. Engineering Acoustics II : Nonholographic Sonic Imaging}

\author{
Donald S. Moseley, Chairman
}

Vitro Laboratories, Silver Spring, Maryland 20910

\section{Invited Papers (30 minutes)}

\begin{abstract}
I1. Ultrasonic Imaging for Underwater Viewing. H. L. WARNER, Naval Coastal Systems Labaratory, Panama City, Florida 32401.-An ideal ultrasonic imaging system for undersea applications is described that would provide diffraction-limited performance at high resolutions $(500<D / \lambda>2000)$ over a field of view of at least $\pm 10^{\circ}$. The image surface of the ideal system would be an array of closely spaced sensors with the size of each sensor being determined by the image spot size. Each sensor would be connected via a discrete processing channel to an element in an electro-optical display. Each processing channel would provide either linear or exponential amplification in specified temporal frequency bands and signal integration with controllable or automatic selection of the integration time. The merits of dividing the passband into three overlapping bands to provide a color display will be explored. The need for broad spatial and temporal frequency bandwidths will be shown. Since an ideal system cannot be built at this time, an examination of the parameters and performance which must be compromised ta obtain practical ultrasonic imaging systems will be made. A number of systems that have been developed for undersea applications will be reviewed.
\end{abstract}

12. Twenty Years of Medical Ultrasonic Imaging. JoHN M. REID, Providence Hospital Seaule, Waskington 98122.-Ultrasonic imaging systems are becoming important in medicine. Our growing understanding of fundamental acoustical-tissue interactions may contribute important new abilities. Absorption and velocity of the sound vary with the structure of tissue and together determine the resolution and penetration of any diagnostic system. The reflecting and scattering properties of tissues are little known although much can inferred from clinical studies. Quantitative work in scattering is only beginning. Imaging systems can be classified on the basis of their range-versus-depth resolution and scanning time-versus-complexity tradeoffs. Early pulse-echo scanners had such "new" features as real-time imaging and gray scale response. Many medical centers now use contact and waterbath scanners. Newer pulse-echo and $C W$ systerns require complex electronics that were not feasible before integrated circuits were available. A promising new area is use of special target properties to identify reflecting objects. For example, working systems identify the Doppler-shifted backscatter from moving blood to map areas of flow. Present systems are adaptable to such imaging techniques. 
13. The Design of Lenses for Ultrasonic Imaging. P. S. GreEn and G. J. Ellers, Stanford Research Institute, Menlo Park, California 94025.-The materials available for the construction of acoustic lenses provide a far wider choice of physical constants than one has in optics. As yet, little effort has been made to design lenses that effectively combine these materials to provide improved imageforming capability. The use of a digital computer for lens design can greatly farilitate such investigations. In this paper we present the results of a study of both simple and compound acoustic lenses, demonstrating the aberrational characteristics and overall merit of lens configurations optimized for a variety of materials and combinations. Also discussed are the effects on image resolution of lens apodization, and the results of experiments with coated acoustic lenses that exhibited considerably reduced surface reflections. [This work has been supported in part by a grant from the National Institutes of Health.]

I4. Experiments in Ultrasonic Imaging for Weld Inspection. W' R. TURNER, Aulomalion Industries, Inc., Vitro Laboratories Division, Silver Spring, Maryland 20910.-As part of a program for investigation of ultrasonic visualization techniques, a laboratory imaging system was used to determine some practical factors in weld inspection applications. The system used the Vitro-designed ultrasonic image tube, and experiments were performed both with and without imaging lenses. The test material was ${ }_{7}^{2}$-in. steel plate containing a butt weld with flaws that had previously been radiographed for reference. Ultrasonic energy was introduced into the plate as a shear wave through a coupling block. Scatter from defects was detected on a monitor as a two-dimensional image against a dark background. Image distortion and phantom images were investigated in detail. System performance criteria for a high data rate inspection system were established, and system modifications were specified for meeting these criteria.

I5. High Resolution Ultrasonic Scanning System. M. J. HuRwITz, Westinghouse Research Laboralories, Pittsburgh, Pennsylvania 15235. - We have built and tested several focused-arc ultrasonic transducers for use in nondestructive characterization of haws in thick metal sections. These transducers transmit and receive ultrasonic pulses at frequencies 2.25 or $4 \mathrm{M} \mathrm{Hz}$ and produce diffraction limited beams at all ranges including the classical nearfield region since the beams are essentially in focus at all ranges. We have used both contact and immersion transducers in conjunction with a mechanical scanner and display package and have produced B-scan images of holes and flaws from 1- to 20-in. depths into metal specimens. Images of patterns of drilled holes have been made demonstrating the resolving of $\frac{1}{32}$ - in.-diam holes about $\frac{1}{16}$ in. apart through $2 \frac{1}{3}$ in. of aluminum. Several other ultrasonic images and comparisons of these with other NDT techniques will be shown. [This work has been supported by the U.S. Navy under contract.]

\title{
Contributed Paper (12 minutes)
}

I6. Experimental UItrasonic Image Converter to Permit a Diver to See in Turbid Water. A. L. RolLE, Engineering Department, Naval Coastal Systems Laboratory, Panama City, Florida 32401.--An experimental ultrasonic image converter was developed to generate a television-like image inside a swimmer's facemask of a $60^{\circ}$ viewing sector from $14 \mathrm{in}$. (arms length) to a maximum range of $10 \mathrm{ft}$. The converter consists of an array of transducers and a similar array of light emitting diodes affixed to opposite ends of a rotating cylinder containing the processing electronics. The transducer array both generates and receives high-frequency ultrasound which is focused by a miniature liquid lens to form multiple narrow beams. The processed transducer outputs are applied to the diode array to form the visual inage, which is conducted to the facemask by fiber optics. Rotating the arrays effects both the scanning of the narrow beams over the object plane and the required synchronous display scan to form the visual image. Tests using hand tools, metal plates, and cylinders demonstrated the feasibility of generating useful images of even specularly reflecting objects with techniques applicable to free-swimmer equipment.

Tuesday, 28 NOVEMBER 1972

DOMINION ROOM, 2:00 P.M.

\section{Session J. Shock and Vibration II : Structural Response and Failure Mechanisms Under Stationary and Nonstationary Random Excitation-Part 2}

\author{
Charles T. Morrow, Chairman \\ Advanced Technology Center, Dallas, Texas 75222 \\ Invited Papers (25 minutes)
}

J1. Time Dependent Root-Mean-Square Response to Nonstationary Random Ercitations. GaRY C.

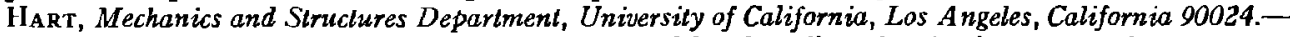
The nonstationary $\mathrm{rms}$ response of single-degree-of-freedom linearly elastic structural systems to several classes of nonstationary random excitations are summarized and compared. Particular emphasis is placed on shot noise, time-modulated white and correlated noise, and evolutionary and segmented nonstationary excitations. The influence of both structural system and nonstationary excitation parameters upon rms response is evaluated. Approximate response approaches are discussed.

J2. Spectral Representations under Nonstationary Random Excitation. W. D. MARK, Bold Beranek and Newman Inc., Cambridge, Massachusetts 02138. - The essential concepts and equations associated with the spectral analysis of stationary random processes are the power spectral density, the auto- 
correlation function, their mutual relationship known as the Wiener-Khintchine theorem, and the power spectrum input-response relationship for linear systems. These concepts and equations can be extended in a natural and unified way to nonstationary random processes. To provide these extensions, it is shown that two time-dependent power spectrum definitions are required-both of which reduce to the same definition in the case of stationary processes. One of these spectrum definitions is essentially equivalent to the class of time-dependent spectra obtained by narrow-band filtering; hence, this definition possesses a clear physical interpretation. However, it does not possess a simple inputresponse relationship for linear systems. The second spectrum definition is the Fourier transform of a time-dependent autocorrelation function. Realizations of this second spectrum exhibit spurious behavior-e.g., they may be partially negative; however, this second definition possesses a simple, exact input-response relationship for linear systems. The exact mathematical relationship between the two spectrum definitions is explained and interpreted in terms of the uncertainty principle for Fourier transforms.

J3. Fatigue and Crack Propagation under Nonstationary Random Excitation. M. ShrnozukA, Department of Civil Engineering and Engineering Mechanics, Columbia University, New York, New York 10027 - The prediction of fatigue life of a structure subject to a dynamic stress history is one of the most difficult engineering problems because of the complexity of the fatigue phenomenon, which is extremely sensitive to material property, structural configuration, stress history, environmental conditions, etc. Recently, engineers have come to a realization that for a more reliable prediction of fatigue life, it is of utmost importance to carry out fatigue tests and perform fatigue analyses under the stress history as close as possible to the reality. This has made it necessary to apply the notion of the random process theory to identify the stress history and to establish the relationship between the fatigue life and the stress history thus identified. So far, most work along this line has been concerned with the stress history that is identified as stationary random process. In this presentation, attention will be focused on those studies in which the nonstationarity of the stress history is considered in the fatigue life prediction.

J4. First Passage Problem in Nonstationary Random Vibration. J. N. YaNG, Jet Propulsion Laboratory, Pasadena, California 91103.--In nonstationary random vibration, a problem of considerable practical importance is to determine the probability, called first passage probability, that the absolute value of the structural response passes out of a threshold for the first time, within a specified time interval. A variety of analytical approximations to the first passage probability are described and compared with results of simulation. Emphasis is placed on nonstationary excitations with short duration, such as earthquake, atmospheric turbulence, spacecraft excitation, etc. First passage probabilities for nonstationary envelopes of the structural response are also discussed.

\section{Contributed Papers (10 minutes)}

J5. On Fatigue Life Under Stationary Gaussian Random Loads. R. TALREJA, Department of Solid Mechanics, Technical Universily of Denmark, Lyngby, Denmark.-Power spectra are taken to represent stationary Gaussian random loads. Location, scale, and shape parameters are defined for power spectra and proposed as a convenient set of load parameters for random loads. The center frequency of a power spectrum, defined as its weighted average frequency, is proposed as a measure of fatigue life. A servohydraulic closed loop testing machine is used to load specimens of carbon steel under six different power spectral shapes. Test results are utilized to evaluate a fatigue life function formulated in terms of the load parameters. The concept of a shape operator introduced by Weibull for probability distributions, is employed to correlate fatigue lives under different power spectral shapes. Good correlations in the test results are obtained.

J6. Panel Session. H. Himeldlau, Chairman.

Tuesday, 28 November 1972

Silver Chimes East, 2:00 P.M.

Session K1. Speech Communication IIa : Communication by Primates

Philip Liegerman, Chairman

Department of Linguistics, University of Connecticut, Storrs, Connecticut 06268

\section{Invited Papers (25 minutes)}

KI-1. Speech Producing Abilities of Primates and Human Evolution. DenNis H. KLATT, Massachusetts Institute of Technology, Cambridge O2139, Philip LiLberman, The University of Connecticut, Stavrs, Connecticut 06268, and Haskins Laboratories, New Haven, Connecticut 06510, and Edmund S. Chelin, Yale University School of Medicine, New Haven, Connecticut 06510.-Comparative anatomic and acoustic studies of the supralaryngeal vocal tracts of living and extinct primates indicate that speech production has been an important factor in human evolution. The sounds of human speech make human language a rapid medium of communication through a process of speech "encoding." The presence of the human vowels $/ a /, / i /$, and $/ u /$ facilitates this process. The supralaryngeal vocal tracts of 
newborn Horno sapiens and the living nonhuman primates are similar and resemble the reconstructed vocal tracts of fossil Australopithecine and Neanderthaloid hominids. Vocal tract area functions that

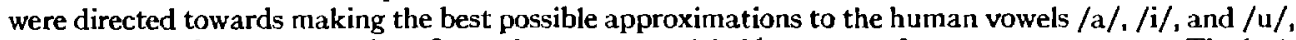
as well as certain consonantal configurations, were modeled by means of a computer program. The lack of these vowels in the phonetic repertoires of these creatures, who lack a pharyngeal region like that of adult Homo sapiens, may be concomitant with the absence of speech encoding and a consequent linguistic ability inferior to modern man.

K1-2. Coding of Species-Specific Vocalizations by the Squirrel Monkey Auditory Corter. JOHN D. Newman, National Institute of Child Health and Human Development, Bethesda, Maryland 20014.Single neurons in the auditory cortex of squirnel monkeys (Saimiri sciureus) were tested with taperecorded vocalizations representing this species' six major call groups. A small minority of these cells were highly selective, responding only to one or two call types. For these cells, a role in categorizing discrete call types appears likely. Some selective cells behaved as "tuned filters," by virtue of the correlation between the best frequency of steady tone bursts for a cell and the major acoustic energy in the effective call type. Most of the neurons responded to several vocalizations. A simple scheme of "feature extraction" does not appear to explain readily the responses of these cells : (a) calls effective for a given cell usually represented structurally different call groups and did not share the same simple acoustic features; (b) many cells responding across call group boundaries did not respond to all the calls of an effective call group; (c) while most of the neurons responded to steady tone bursts, their responses to tones were generally not satisfactory indicators of their responsiveness to vocalizations ; (d) in some cases, the nature of a cell's response was shown to depend upon interactions of nonadjacent parts of a vocalization.

K1-3. Physiological Control of Vocalizations in the Japanese Monkey : Inferences from a Field Study. Steven Green, The Rockefeller University, New York, New York 10021.-A field study of vocal communication in the Japanese monkey reveals their repertoire to be composed of patterns that intergrade acoustic features. If the repertoire is divided into classes on the basis of a few distinctive features, a regular pattern of association is evident between these classes and the social situation in which they are uttered. The association is not arbitrary. It can be largely accounted for by the internal state of the animals. Uniformly tonal sounds, for example, are given only by unaroused monkeys and are used in situations of affinitive contact. On the other hand, uniformly atonal sounds are given by very agitated animals and accompany aggressive threats. Within the broad framework of covariation of classes of sound with internal state, there are opportunities for small differences between closely related vocal patterns to be utilized socially. Evidence is presented that these opportunities are exploited in a number of ways; for example, minor variations of vocalizations within a single class mirror fine nuances in social usage. The acoustic nature of these minor signal variations can also be interpreted as graded reflections of the physiological-emotional substrate of the vocalizing animal.

K1-4. Reflections on Recent Efforts to Teach Chimpanzee Language. David McNeILl, University of Chicago, Chicago, Illinois 60637.--A review of two recent experiments in which chimpanzees have been taught forms of human language supports the conclusion that chimpanzee shares with man a number of basic linguistic and conceptual characteristics. In spite of these similarities, however, chimpanzee has difficulty with those aspects of linguistic structure that encode conceptual relationships (such as agent and action), and instead spontaneously adopts a novel form of syntax based on social relationships (such as addressee and nonaddressee). It is argued that this difference between man and chimpanzee reflects a difference in species endowment, the two species having a syntax that encodes distinctly different kinds of information, which can be traced to differences in life conditions. It is conceivable that free ranging chimpanzees are already using a linguistic system organized along the lines revealed in the experimental studies of language acquisition by chimpanzee.

Tuesday, 28 Novemier 1972

Silver Chimes East, 4:00 P.M.

\section{Session K2. Speech Communication IIb : Communication in Cetacea}

Harry Hollien, Chairman

Communication Sciences Laboratory, University of Florida, Gainesville, Florida 32601

Invited Papers (times as indicated)

4:00

K2-1. Dolphin Communication. David K. Caldwell, Communication Sciences Laboralory, University of Florida, Gainesville, Florida 32601.-A history of research and writing (the two not necessarily synonymous) regarding dolphin communication will be presented along with a summary of the present 
trends in thinking regarding this somewhat controversial topic. The primary emphasis will be placed on the "vocal" aspects of dolphin communication, but other methods of communication in this mammalian group will be outlined; i.e., such things to be discussed are non-"vocal" sounds such as the jaw clap and tail slap, body postures and positions, tactile contact, and pigmentation patterns. Signal detection systems in the dolphin will be considered as they relate to the apparent signal emission systems. Behavioral correlates will be outlined for many of these communication modes, and various experiments described that bear on the matter of whether or not presumed communication modes are indeed used for this purpose. Special attention in this regard will be given to the whistle component of the communication process.

$$
\text { 4:45 }
$$

K2-2. Auditory Processing by Dolphins of Signals Related to Signature Whistles. HARry Hollien and MelaA C. Caldweld, Communicalion Sciences Laboralory, University of Florida, Gainesville, Florida 32601. - It is now well established (Caldwell and Caldwell, 1970) that one of the major formsif not the major form-of dolphin communication is the signature whistle. In an effort to obtain information concerning the types and levels of auditory discriminations dolphins must be able to make if they are to process these calls, an extended series of related studies is heing conducted. In the first investigation, the research focus was on auditory pattern recognition within three domains: those of frequency, time, and acoustic configuration. Specifically, a "master" dolphin whistle was patterned and synthesized-as were three other whistles varying only with respect to their frequency, temporal, or spatial characteristics, respectively. Additionally, sets of synthesized whistles were obtained for several equally spaced intermediate points along each of the three experimental continua : all signals were generated through the courtesy of the Speech Transmission Laboratory, Royal Institute of Technology, Stockholm, Sweden. For the three substudies, an experimental animal (an Atlantic Bottlenose dolphin) was administered 50 trials (25 stimuli each) of the "master" whistle and one of the experimental stimuli. Following completion of this procedure, a second set of 50 trials would compare the master whistle with another set of experimental signals; these sets were always ordered from the most different comparisons to the least different within a substudy. Once all data were obtained from one substudy (frequency, say), the whole procedure was replicated for the next. With one exception, the experimental animal was able to discriminate between all stimuli-even those that were almost identical. A discussion of these results and a review of the direction of subsequent research will be provided.

\title{
5:05
}

K2-3. A Comparison of Mysticete and Odontocete Cetacean Acoustic Signals and Their Behavioral Consequences. Howard E. WinN, Graduate School of Oceanography, University of Rhode Island, Kingston, Rhode Island.-Odontocete cetaceans produce echolocation clicks, whistles, cries, chirps, and similar sounds. Mysticete cetaceans produce low-frequency moans, grunts, and thumps, and at least one species produces cries and chirps. The production of clicks by mysticetes has been controversial to date, but recent evidence suggests they emit pure frequency clicks up to $25 \mathrm{kHz}$. Mysticetes do not produce whistles. The organization of sounds implies that odontocetes use the highfrequency clicks for capture of individual prey (fish and squid). Mysticetes may or may not use their clicks for feeding, but if so they may be used only to locate masses of plankton and fish-not in locating single individuals. Again, the variety of signals produced by odontocetes in all seasons suggests a more intricate social organization. The complicated song of the humpback whale, produced only three months of the year, may have a wide variety of functions, but suggests a less complicated social organization outside of this season. Some of the low-frequency sounds of this mysticete may be needed for long-range communication, perhaps related to their extended migration.

TUESDAY, 28 NOVEMBER 1972

Burgundy East, 2:00 P.M.

\section{Session L : Psychological Acoustics I : Masking and Loudness}

\author{
Charles S. Watson, Chairman
}

Central Institute for the Deaf, St. Louis, Missouri 63110

\section{Contributed Papers (10 minutes)}

L1. Perception of Temporal Order for Tones. R. W. PEters AND T. J. WoOD, Institute of Speech and Hearing Sciences, University of North Carolina, Chupel Hill, North Carolina 27514. - Judgments of temporal order were obtained for three successive pulses, $500-, 1000-$, and $2000-\mathrm{Hz}$ tones, for various durations and intervals between pulses. The durations were $20,40,80$, and $160 \mathrm{msec}$ and the intervals were 0,40 , and $80 \mathrm{msec}$. Signal presentations were monaural with both signals and the collection of responses under computer control. The results indicated a bimodel distribution in that some 
individuals were of ten able to detect correct order even for the shorter durations and intervals. More typical, however, were responses that approached, but did not reach, complete accuracy for the longer durations and intervals. In fact, at the longest duration of $160 \mathrm{msec}$, most listeners only achieved about $70 \%$ accuracy. The results will be discussed relative to the interaction of pulse duration and interval as it relates to judgments of order [This research was supported, in part, by the University Research Council of the University of North Carolina.]

L2. Models of Attention for Vibrotactile Stimuli. JAMEs C. Craig, Richard M. Shiffrin, and Eli Cohen, Department of Psychology, Indiana University, Bloomington, Indiana 47401. - The study of summation for vibrotactile stimuli between two loci on the skin has produced conflicting results. Craig [Perception Psychophys. 4, 351-354 (1968)] found evidence for such summation while Franzen, Markowitz, and Swets [Perception Psychophys. 7, 193-196 (1970)] did not. To account for their data, Franzen $e t$ al. proposed a singlechannel model of attention for weak vibrotactile stimuli. The present experiment was designed to test models of attention for the skin. Two conditions were used to evaluate observer's ability to detect near threshold vibrotactile stimuli. In both conditions, a sigual was presented at one of three skin loci, the observer stating which locus received the stimulus. In the simultaneous condition, there was a single observation interval during which a signal occurred at one of the three loci. In the sequential condition, there were three observation intervals presented successively with a signal presented at one of the three loci during one of the three observation intervals. Each observation interval was associated with a particular locus. Theories postulating attentional control predict performance to be inferior in the simultaneous condition since that condition requires sharing of attention among the three possible sources of tactile stimulation. The data showed performance to be identical in the two conditions. The results indicate that a single-channel model is not appropriate and, in addition, argue for an unlimited-capacity nonattention model of tactile perceptual processing. [Research supported by $\mathrm{NIMH}$ and NIH.]

\section{L3. Temporal Modulation Transfer Functions for Audition.} Neal F. Viemeister, Universily of Minnesota, Minneapolis, Minnesota 55455.- Systems analysis has been extensively used to study the spatial and temporal processing of visual inputs and recently has been applied to auditory filtering. Two techniques are discussed in the present paper, which extend this approach to auditory temporal processing. In the "modulation-threshold" procedure, the threshold modulation amplitude of a sinusoidally modulated waveform (either sinusoids or noise) is determined. The function relating this threshold to modulation frequency is a temporal modulation transfer function (TMTF) and ideally is the attenuation characteristic of the temporal processing system. TMTFs obtained with this procedure show only low-pass filtering and are $3 \mathrm{~dB}$ down at approximately $10 \mathrm{~Hz}$. In the "temporal probe technique," the threshold amplitude of a brief probe (either a bandpass filtered or a wide-band pulse) is determined at several temporal locations in a noise masker whose amplitude is sinusoidally modulated. The function relating probe amplitude to its time of occurrence (the masking curve) can be well-fitted by a sinusoid with a dc component. The amplitude spectrum (TMTF) and phase spectrum are determined from masking curves obtained at several modulation frequencies. The empirical TMTF indicates a shorter time constant and steeper attenuation rates than those obtained with the modulation-threshold procedure. [This research was supported by the National Institutes of Health and by a Special Traineeship from NINDS.]
L4. Reaction Time as a Measure of Suprathreshold Masking. R. N. Lanson, T. Garrett, and V. Matsuo, Department of Psychology, Queens College, Flushing, New York 11367.Reaction time (RT) to pure tones masked by white noise was determined with two observers. At least five noise intensities, covering a 0-80 dB SL range, were selected; the tone intensity range was similarly divided. Seven tone frequencies from $250 \mathrm{~Hz}$ to $8 \mathrm{kHz}$ were investigated. Finger-withdrawal $\mathrm{RTs}$ to tone onset were taken on 30 successive light-cued trials at a given combination of frequency and tone and noise intensities, presented monaurally. Noise was present continuously; tone duration was $1 \mathrm{sec}$, and foreperiod was variable. Data were obtained for intensity pairs yielding less than 1 sec RTs. Noise intensities were run in increasing order; tone intensities varied randomly within a given noise level. At equal signal-to-noise ratios for a given frequency, functions relating RT to tone and noise intensity appeared similar. Equal RT contours showed threshold shifts as a function of noise intensity. Comparison of these results with threshold masking data illustrated the use of RT as a measure of suprathreshold masking.

L5. Predicting the Detectibility of Complex Signals. W. J. Tanski And F. A. Andrews, Acoustics Group, The Catholic University of America, Washington, D. C. 20017.-A series of investigations have been conducted to determine to what extent one may predict the aural detectibility of a complex signal in a background of masking noise. Observers were tested binaurally using the two-alternative forced-choice procedure to obtain psychometric functions for simple signals. Modeling the auditory system as an energy detector preceded by a set of bandpass filters, and knowing the statistics of the masking noise and signal plus noise distributions, one may determine the difference distribution statistic upon which the auditory system bases its decisions. The psychometric functions allow one to determine the inefficiency factor for the auditory system and, combining this knowledge with the difference distribution statistics, it is possible to develop a procedure for predicting the detectibility of various complex signals. The results of tests with signals consisting of various combinations of tones and noise will be compared with predictions. Limitations will be discussed.

L6. Implications of Peripheral Frequency Selectivity for Nonsimultaneous Masking. H. DuIfHuIs, Institute for Perception Research, Eindhoven ihe Neiherlands. ${ }^{*}-$ Frequency analysis at the level of the VIIIth nerve is so sharp that onset and offset effects in responses to short signals produce a nonnegligible extension of the signal duration. Therefore, excitation patterns produced by signals that were separated in time, can overlap. The overlap of excitation patterns can produce masking, in simultaneous as well as in nonsimultaneous conditions. It is suggested that this peripheral part of the masking process can be held responsible for the major part of backward masking and for a part of forward masking. We refer to the above masking as transient masking. The finding that backward masking decreases with increasing probe frequency favors this interpretation.

* Present address: Research Laboratory of Electronics, MIT, Cambridge, Mass. 02139

L7. Aural Harmonics: Nonlinearity and Loudness. J. ERdreich aND T. D. Clack, Department of Otorhinolaryngology, Kresge Hearing Research Insitute, University of Michigan Medical School, Ann Arbor, Michigan 48104.-An inverse relation between the loudness of a $1000-\mathrm{Hz}$ pure tone and the amplitude of the second aural harmonic it generates was reported last year. To investigate more rigorously, normal 
listeners $(N=12)$ produced individual absolute loudness scales from ratio (doubling and halving) and equisection (five points over each of three ranges) judgments. These scales have been interrelated using Garner's [J. Acoust. Soc. Amer. 26, 73-88 (1954)] method of corrected ratios. Estimates of the second and third aural harmonics of a $500-\mathrm{Hz}$ pure tone were made from tone-on-tone masking measurements. A comparison of the doubling and halving ratios will be discussed together with the shunting hypothesis: i.e., energy shunted into harmonic production is subtracted from that available lor conversion into the magnitude of the loudness sensation.

L8. Differential Reinforcement of High Responding Rate with Changes in Sound Intensity as the Reinforcer. J. A. Molino, Institute for Basic Standards, Nabional Bureau of Standards, Washington, D. C. 20234.-Subjects (19 high school students) worked on a modified DRH schedule by tapping on a telegraph key to reduce the intensity of a continuous acoustic stimulus. The stimuli were three pure tones $(125$, $1000,8000 \mathrm{~Hz}$ ) and a white noise presented diotically through earphones. Every 20 responses falling within $200 \mathrm{msec}$ of each other praduced a 1-dB decrement in stimulus intensity. Failure to respond produced an intensity increase of $1 \mathrm{~dB}$ every $4 \mathrm{sec}$. One of the stimuli was chosen at random for each 10-min session until four sessions had been completed with each stimulus. The starting intensity level was always 70-74 $\mathrm{dB}(A)$. The average intensity curve as a function of time stabilized after about $4 \mathrm{~min}$. The asymptotic levels achieved after $7 \mathrm{~min}$ were taken as a measure of equal averseness for the stimuli. Equal averseness levels were compared with other subjective weighting contours: equal loudness level, $\mathrm{dB}(A)$, and perceived noise level. The equal aversiveness levels fell between $\mathrm{dB}(A)$ and the other contours.

L9. Loudness Evaluations of the Sound from an Electric Clock. W. M. Viebrock* and M. J. Crocker, Ray W. Herrick Laboratories, School of Mechanical Engineering, Purdue University, West Lafayette, Indiana 47907.-The noise from a consumer electric clock was studied to evaluate loudness estimating procedures. Eight different clock noises were tape recorded for presentation to a panel of people for loudness judgments. An electronic switch enabled the recorded clock noise and a $1000-\mathrm{Hz}_{\mathrm{z}}$ tone to be presented alternately via earphones for $f$-sec durations with $\frac{1}{-}-\mathrm{sec}$ silences in between signals. The person adjusted the $1000-\mathrm{Hz}$ tone to match the loudness of the clock noise providing a correlation to the phon loudness scale. It was determined that Steven's method generally underestimates the loudness but is usually less than $10 \mathrm{~dB}$ in error. If ranking on a relative loudness scale is all that is desired, $\operatorname{dB}(A)$ measurements will suffice. [This work was supported by Westclox Division, General Time Corporation, LaSalle, Illinois.]

* Present address: Lockheed Missile \& Space Co., Sunnyvale, Calif.

L10. Temporal Summation for Tones at Threshold. H. N. WRIGHT, Department of Ololaryngology, State University of New York, Upstate Medical Cenler, Syracuse, New York 13210.- The threshold for tones as a [unction of their duration $(500,200,100,50,20$, and $10 \mathrm{msec})$ was investigated with the tracking method in a population of $\mathbf{5 0}$ normal-hearing listeners (25 male, 25 female; 25 right ears, 25 left ears) at nine frequencies from 125 through $8000 \mathrm{~Hz}$ under test-retest conditions. The results indicate that temporal auditory summation, at least as measured with the tracking method, conforms with theoretical expectations. There was no variation in the function with frequency, sex, or ear. When the thresholds for individual listeners are normalized to the threshold for 20 -msec tones, the variability both among and within subjects is about the same at all frequencies for all duration tones (standard deviation of $2 \mathrm{~dB}$ ). These results permit further investigation on the effect of other variables (e.g., hearing impairment) on the threshold for tones as a function of their duration. [Supported by a PHS Research Grant [rom NINDS.]

L11. Equal Loudness Pressures and the Normal Ear. JoHN A. Victoreen, Vicloreen Laboratory, Maitland, Florida 32751.-Measurements are presented to show equal-Joudness sound-pressure values for a number of ears. Observations are given as average values for a number of ears that are useful in defining how a "perfect average" ear would be expected to perform. Equal-loudness sound-pressure observations are also given for several individual ears showing how supposedly "normal" ears deviate from a "perfect average" normal ear. Measurements were made by means of damped wavetrain signals having a decrement of 0.90 . Signals were applied to the ear by means of a Sointrex HA10 circumaural earphone, which was calibrated on a flat-plate coupler. A number of conclusions are suggested which relate this type of measurement to the behavior of the average normal ear as a standard of general comparison without attempting to define specific numerical values.

L12. Measurement of Loudness Adaptation with a Monaural Procedure. T. L. WILEy, Department of Communicative Disorders, The University of Wisconsin, Madison, Wisconsin 53706, and A. M. SMall AND D. J. Lilly, Department of Speech Pathology and Audiology, The University of Iowa, Iowa City, Iowa 52240.-Monaural loudness-adaptation measurements were made at several suprathreshold levels for 500 and 4000 $\mathrm{H}_{2}$ with normal-hearing subjects. A unique procedure used the subject as a null device and adaptively varied the signal level over time until no change in loudness was indicated. Constant-loudness functions (SPL with time) were derived for each condition over a 30-sec interval. The results indicated no monaural loudness adaptation. This generalization held for all experimental conditions. These findings support similar data obtained by other investigators using delayed-balance procedures. It was concluded that the adaptation effects observed in experiments using some type of simultaneous binaural stimulation apparently are due to binaural interaction and may reflect central as well as peripheral infuences.

L13. A Timing Model for the Intensity and Frequency Discrimination Functions. R. Duncan LuCE, School of Social Sciences, Universily of California, Irvine, California 92664, AND David M. GREen, Universily of California, San Diego, La Jolla, California 92037.-For a pure-tone signal of intensity $I$ and frequency $f \leq 1000 \mathrm{~Hz}$, we attempt to arrive at the dependence of $\Delta I$ and $\Delta f$ on $I$ and $f$. Single fiber neural data for low frequency tones suggest that the pulse trains are approximately renewal process with interarrival times of the form IAT $-I / f+X$, where $I$ is a geometrically distributed random variable with parameter $p$ and $X$ is a bounded random variable with $E(\mathrm{X})=0$ and $V(\mathrm{X})$ proportional to $1 / \mathrm{f}^{2}$. Because the distribution of $I A T \mathrm{~s}$ is almost perfectly multimodal, if the sample is reasonably large, it is usually possible to determine the value of I for each $I A T$. Since $E(\mathbf{I A T})=1 / p f$ and $E(I A T / I)=1 / f, p$ and $f$ can be estimated from the sample in the obvious way. 'Using a $d^{\prime}$ argument, we derive $\Delta P$ and $\Delta f$ as functions of $p$ and $f$. Assumptions are made relating $p$ and the sample size to $I$ and $f$, and from these assumptions are derived formulas relating $\Delta I$ and $\Delta f$ to $I$ and $f$. These predictions are compared with the existing data. Various theoretical problems are pointed out. [This research was supported by a grant from National Science Foundation.] 


\title{
Session M. Psychological Acoustics II : Pitch, Protectors, Perception, and Instruments
}

\author{
Arnold M. Small, JR., Chairman \\ Department of Speech Pathology and Audiology, University of Iowa, \\ Iowa City, Iowa 52240
}

\section{Contributed Papers (10 minutes)}

\begin{abstract}
M1. Examples of Spectral Analysis Techniques for Analyzing Speech and Hearing. Charles E. Schmid, Honeywell Inc., Marine Systems Center, 5303 Shilshole Avenue N. W., Seatlle, Washington 98107.--Various stationary and nonstationary spectral analysis methods are utilized to analyze two-channel data obtained from the ear of an anesthetized guinea pig. One channel was recorded from a microphone placed in front of the ear, while the second was simultaneously taken from an electrode on the round window of the cochlea. A stationary white Gaussian acoustic source was used to obtain an estimate to the transfer function of the ear. This was accomplished through digital computer calculations of the auto and crosspower spectral density functions, along with the coherency function. Linear phase changes and resonances were observed in the frequency regions exhibiting high coherency. A spoken sentence was utilized as a nonstationary source. The balance between frequency resolution and time definition is illustrated on various displays of time-varying spectra. They include two-dimensional, three-dimensional, and ordinary spectrogram types of displays.
\end{abstract}

M2. Can Amplitude Compression Secure a Distortionless Amplification in Hearing Aids? IGOR V. NÁĚLLEK, University of Maryland, Speech and Hearing Science, College Park, Maryland 20742.- It is generally believed that compression can limit the power output of a hearing aid without adding distortion to the signal. The occurrence of distortion is the main objective against limitation of output by clipping. However, similar distortion can occur also with amplifiers with compression. If the compression reacts to the increase of the input signal with a delay, during such a delay the gain is not reduced, and two possibilities arise: either even a strong signal is fully amplified and no protection occurs, or the amplifier is overloaded and the signal distorted. In the later case, compression reduces the time interval of distortion of strong signals as compared to distortion durations without compression. The duration of distortion can be reduced below perceptable level by the use of a very short attack time. Short release time also needed for short-term dynamic range reduction can have some negative consequencies. Requirements on different parameters of compression for various conditions of use will be discussed. and several examples of responses of hearing aids with compression shown.

M3. A Modest Computer Psychoacoustic System. Richa RD A. Campbell, Audiology and Speech Palhology, Velerans Administration Hospital, Miami, Florida 33125.-A psychoacoustic system, based on a minicomputer (Data General, Nova 1200) is described in which the computer not only controls attenuators and gates but also generates the sinusoidal stimuli by waveform reconstruction. Representative harmonic distortion values for the octave frequencies 125 $8000 \mathrm{~Hz}$ (with a digital output rate of $32000 / \mathrm{sec}$, and eightbit conversion, and a $16000-\mathrm{Hz}$ low-pass filter) range from $0.08 \%$ to $0.4 \%$, respectively. The psychophysical procedure used involves the random selection of stimulus frequency, undefined random observation intervals, a yes-no response, which is classified into one of eight categories (Hit, Miss, six kinds of False Alarms) with six possible results of each on the up-down adaptive method, on each trial. Despite the rather complex procedure being utilized, use of the direct memory access and interrupt capabilities of the computer result in the processor being idle about $95 \%$ of the time. The approach to auditory stimulus generation taken in this system, which minimizes the interfacing and programming attention required for basic stimulus generation, is seen as a powerful aid to many kinds of psychoacoustic research.

M4. Beats of a Sinusoidally Amplitude-Modulated Signal with a Tone near the Modulation Frequency. T. P. KERR, Kresge Hearing Research Instilute, Universily of Michigan, Ann Arbor, Mickigan 48104, and H. H. Mikaelian, Department of Psychalogy, University of Georgia, Alhens, Georgia 30601. - The "residue pitch" produced by a train of dc pulses will not beat with a search tone of similar frequency (Schouten, 1938). These results have been interpreted to mean that combination tones do not augment the residue pitch generated by low-amplitude periodic waveforms. This assumption was reexamined for the case of a sinusoidally amplitudemodulated (AM) signal synthesized from three inharmonic components-2050,2400, and $2750 \mathrm{~Hz}$. Acoustic energy at the modulation frequency $(350 \mathrm{~Hz})$ was $60 \mathrm{~dB}$ lower than the $2400-\mathrm{Hz}$ component. The modulated signal was presented at $35,45,55$, or $65 \mathrm{~dB} \mathrm{SPL}$, together with a $352-\mathrm{Hz}$ search tone. Subjects adjusted the search tone amplitude for "best beats." Two of the five subjects could detect beating at 350 $\mathrm{Hz}$ when the AM signal was less than $35 \mathrm{~dB}$ above threshold. When the search tone was set to $35 \mathrm{~dB}$ SL and above, all subjects heard a periodic sweeping pitch, located in the same approximate spectral region as the $\mathrm{AM}$ primary tones. This phenomenon, not previously described, will be discussed in relation to residue and place theories of pitch. [Experiments performed at Submarine Medical Research Laboratories, Groton, Connecticut, under ONR Contract with Connecticut College.]

M5. Effects of Partial Hamming Function Modulation of Tone Bursts. Robert B. MaHAfFey, Institute of Speech and Hearing Sciences, University of North Carolina, Chapel Hill, North Carolina 27514. - Some perceptual effects of Hamming function modulation of pure-tone bursts of short duration were presented at an earlier ASA meeting [Spring 1967]. In the present studies, subjects' pitch percepts were measured and thresholds were obtained for tone bursts having contours of the following descriptions: (1) full Hamming function modulation; (2) full rectangular function modulation; (3) first half-Hamming function modulation, last half-rectangular function modulation; and (4) first half - rectangular function modulation, last half-Hamming function modulation. The full-cycle Hamming function bursts provided the least 
variance in pitch perception measurements and the highest thresholds. The rectangularly modulated tone bursts afforded the greatest variances in pitch measurements and the lowest thresholds. The effect to tone bursts having contours beginning as Hamming functions and ending as rectangular functions approached those of the full Hamming function modulations, whereas the effects to the tone bursts having contours beginning as rectangular functions and ending as Hamming functions approached those of the rectangular bursts. On the basis of preliminary data from hearing-impaired patients, clinical implications are cited relative to the threshold shifts obtained with Hamming function bursts as a function of burst duration and of tone frequency.

M6. The Pattern-Transformation Model of Pitch. Frederic L. WIGHTMaN, Institute for Perception-TNO, Soesterberg, Netherlands. - This report describes a new approach to pitch perception, based on a kind of auditory pattern recognition. The general approach is formalized in a mathematical model, the "pattern-transformation model." In the first stage of this model, an acoustic stimulus is assumed to be "transformed" by the sense organ into what is called a neural activity pattern. This pattern is thought to represent roughly the power spectrum of the waveform. Thus, the model is phase insensitive; the temporal fine structure of the stimulus waveform is ignored. The first-stage pattern (power spectrum) is then assumed to be Fourier transformed (neurally) into another pattern of neural activity. This second pattern roughly represents the autocorrelation function of the stimulus. Pitch is derived from the positions of maximal activity in this pattern. From preliminary tests, it appears that the model can accurately predict the pitches of many types of acoustic stimuli. In addition, the model provides numerical estimates of pitch "strength" and "ambiguity." While experimental confirmation of these predictions is lacking, the strength estimates agree at least qualitatively with available data. [Research supported by a National Science Foundation Postdoctoral Fellowship awarded to the author and the Institute for Perception-TNO, Netherlands.]

M7. Prediction of Cubic Difference Tones from a Model of Pitch Perception. A. Ravindran and Ian B. Thomas, Electrical Engineering Department, University of Massachusetts, Amherst, Massachusetts 01002.-Using a model of pitch perception already shown to give accurate predictions of residue pitches and difference tones [83rd Meeting, J. Acoust. Soc. Amer. 52, 141 (A) (1972)], we have been able to predict cubic difference tones for frequency ratios of the primary tones from 1.167 to 1.500 . The model involves the determination of axis crossing intervals of the stimulus at an appropriate basilar membrane (BM) location through Flanagan's BM transfer function, taking into account both amplitude and phase changes. The model notes and marks the time of significant differences in adjacent axis crossing intervals of the signal at the appropriate BM location. Time intervals corresponding to cubic difference tones are found between these time marks. In almost all cases investigated, the axis crossing intervals must be determined at a BM location corresponding to the lower primary frequency. Contrary to our earlier statement, the prediction of cubic difference tones from this model is achieved without any assumption of nonlinear distortion mechanisms. [Work supported by NIH.]

M8. The Reliability and Validity of In-Ear Microphone Measurements of Ear Protective Devices. Thomas L. Nichol.s and J. Bradley Swanson, Pioneering Research Laboralory, U. S. Army Natick Laboratories, Natich, Massachusetts 01760.-A miniature microphone was placed in a subject's ear after the method described by Brammer and Piercy [J. Acoust. Soc. Amer. 52, 1972180 ], and its output fed to a spectrum analyzer set to a bandwidth of $100 \mathrm{~Hz}$. The subjects were seated in a semireverberant room into which a white noise field at $95 \mathrm{~dB}$ SPL was introduced. The attenuation of three ear covering devices was measured by subtracting the spectrum obtained with the ear cover in place from the spectrum obtained with the subject's ears bare. The repeatability of measurements was examined with both small changes in microphone and ear cover placement. Microphone placement proved to be more critical, although correlation coefficients between measures across days averaged 0.88 . Differences between runs measured at selected frequencies between 250 $\mathrm{Hz}$ and $7 \mathrm{kHz}$, generally fell within a range of $\pm 3 \mathrm{~dB}$. Validity was estimated by comparing the microphone data with psychophysical data gathered earlier. The former tended to indicate more attenuation than the latter, though the correspondence between the two was encouraging.

M9. Evaluation of Two Nonlinear Ear Protectors Using a Constant Temporary Threshold Shift Technique. W. D. Ward AND A. LUPBERGER, Hearing Research Laboratory, University of Minnesota, Minneapalis, Minnesota 55455.Ordinary approved methods for assessing the performance of ear protectors are not appropriate for testing nonlinear devices whose reduction of impulse noise is achieved by peak clipping. Instead, the effective protection can be estimated by determining how much more intense a series of pulses must be with the protector in place in order to produce a given temporary threshold shift (TTS). Such tests were made on two commercially available nonlinear devices, using five test subjects. The median effective protection against a 155-dB impulse was about $13 \mathrm{~dB}$ for both devices; however, one of these (a complicated mechanism incorporating a valve that is allegedly closed by the force of the impulse) is five times as expensive as the other (an ordinary V51-R-type plug with a metal insert through which a $1 / 40$-in. hole has been drilled). The results are shown to agree with measurements of peak reduction using cadaver ears.

M10. High Noise Reduction Ear Enclosures. C. H. Allen AND C. 1. MAlme, Bolt Beranek and Newman Inc., Cambridge, Massachusetts 02138.-Personnel associated with operation and maintenance of combat vehicles and military aircraft are subject to noise environments so severe that the available ear protective devices do not provide adequate noise reduction. A study and development program has been conducted to improve available noise reduction by (1) design of an ear enclosure cushion to provide a better seal at low frequencies; (2) provision of a secondary seal inside the earcup between the communications earphone and the ear canal, and (3) utilization of electronic noise cancellation to improve further the noise reduction at low frequencies. The design evolved from this study features a low-profile ear enclosure in which the earcup and cushion form a smooth continuous shell that is curved inward to rest behind the pinna. The cushion is fabricated from material which has frequency-dependent viscoelastic properties. It is compliant at low frequencies to provide a good seal, but stiff at autio frequencies to act as an extension of the earcup wall. The desigu requirements that have been met provide for better than $20 \mathrm{~dB}$ noise reduction at low frequencies and better than $45 \mathrm{~dB}$ at high frequencies. [Supported by the U. S. Army, under contract.]

M11. Noise and Pure-Tone Attenuation of Hearing Protectors. D. F. BolkA AND P. L. Michael, Environmental Acoustics Labaratory. The Pennsylvania State University. University Park, Pennsylvania 16802.-The acoustic attenuation characteristics of five ear muffs and four ear plugs were measured. Ten experienced listeners performed threshold shift determinations using pulsed pure tones and $\frac{1}{3}$-oct bands of noise at $0^{\circ}$ and $90^{\circ}$ incidence in anechoic conditions and 
the same bands of noise in semireverberant conditions. Sound field diffuseness was determined by a directional microphone technique and subjectively. Ear muff attenuation at 1 and $4 \mathrm{kHz}_{2}$ was significantly lower $(p<0.05)$ for the noise bands in diffuse field than for frontal incidence pure tones in free field. This difference was not observed for ear plugs. The subjects agreed that threshold determinations were no more difficult with noise bands in diffuse field than with pure tones in free field; variability was somewhat lower for the noise band stimuli. Physical measurements of ear-muff attenuation on an "artificial head" test fixture exhibited statistically significant rank correlation with diffuse field subjective measurements at test frequencies of $2 \mathrm{kHz}$ and below, although absolute attenuation values showed only fair agreement. The diffuse field subjective 'method and the "artificial head" method are being recommended for ANSI standardization. [This research was sponsored by DHEW, Public Health Service.]

M12. Temporal Order Discrimination: Recognition without Identification by Untrained Subjects. RicharD M. WARREN, Department of Psychology, University of Wisconsin-Milwaukee, Milwaukee, Wisconsin 53201.-Earlier work with unpracticed subjects has indicated that identification (naming) of temporal order for repeated sequences consisting of three or four successive sounds such as hisses, tones, and buzzes could not be accomplished at item durations of $200 \mathrm{msec}$ and below [see Warren et al., Science 164, 586-587 (1969)]. In the present experiment, eight separate groups of 30 subjects were required to tell whether alternating sequences, each consisting of a reiterated presentation of the same three or four successive items, had these items in the same or different order. Identification of these orders was not required. Accuracy of same/ different judgments depended upon details of the experimental procedure employed, but in general was significantly better than chance when all items lasted 200 msec. Extending the duration of each item in one of the two sequences beyond 200 msec made the task more difficult. When one sequence had items at $200 \mathrm{msec}$ and the other at $670 \mathrm{msec}$, same/ different judgments were at chance level. It appears that if identification of orders cannot be accomplished, then recognition of the identity of or difference between patterns requires a prior "temporal template" for comparison of both duration and order. [Research supported by NSF.]

M13. Temporal Order Discrimination: Identification and Recognition by Trained Listeners. Richard M. Warren, Department of Psychology, University of $U^{\prime}$ 'sconsin-Milwaukee, Milwaukee, IVisconsin 53201.-Four trained subjects listened to successive sequence bursts, each burst consisting of three component iterns (filtered $1000-\mathrm{H}_{2}$ square wave; noise band, $2500-\mathrm{Hz}$ tone) repeated several times without pauses between items, until they decided whether alternate bursts had these items in the same or different order. Prior training consisted of listening to known same or different sample sets as requested by the subject until he indicated readiness to receive these sets as unknowns. Same/different recognition was significantly above chance for at least half of the subjects at each of three interburst intervals ( $50 \mathrm{msec}, 1.5 \mathrm{sec}, 3 \mathrm{sec}$ ) for item durations from 5 through 400 msec. With items shorter than 100 msec, the order could not be identified, and judgments were based on pattern "quality." In separate experiments with single stimulus bursts, four listeners were trained by receiving immediate feedback concerning their accuracy in identifying (naming) order. For two item sequences (square wave and noise), correct identification was obtained for item durations from 5 through 400 msec. When these stimulus pairs were preceded and followed by a tone of the same duration as the test items, accurate identification was obtained for item durations from 10 through $400 \mathrm{msec}$. [Research supported by NSF.]

WEDNESDAY, 29 NOVEMBER 1972

EMPIRE ROOM, 9:00 A.M.

Session N. Motor Vehicle Noise

Douglas Muster, Chairman

University of Houston, Houston, Texas 77004

\title{
Invited Papers (30 minutes)
}

\begin{abstract}
N1. Motor Vehicle Noise Spectra, Their Characteristics and Dependence upon Operating Parameters. Ralph K. Hillquist, General Motors Proving Ground, Milford, Michigan 48024, AND William N. Scotr, Chrysler Corporation, Detroit, Michigan 48231. - Present highway motopr vehicle noise levels are presented as a function of operating condition (idle, steady speed, normal acceleration, and coast). The variables that affect vehicle noise level, such as speed, throttle, road, location, and weather are considered. The spectral composition of motor vehicle noise is indicated, .and, where possible, the prevalence and distribution of these levels is stated. The data represent both interior and exterior noise of such highway vehicles as trucks, buses, automobiles, and motorcycles. Some noise abatement approaches are discussed, including an order of priority for vehicle noise limitation, a rationale for light-vehicle maximum noise level, highway considerations, and operator responsibility.
\end{abstract}

N2. Internal-Combustion Engine Induction and Exhaust System Noise. ERICH K. BENDER, Boll Beranek and Newman Inc., Cambridge, Massachusetts 02138, and Anthony J. Brammer, National Research Council of Canada, Ottawa, K1A, OSI Canada.-The importance of induction and exhaust systems on community and passenger noise exposure and engine performance are introduced. Discussion is given of the types of engines and induction/exhaust systems they use. They include naturally aspirated two- and four-stroke engines, supercharging, turbocharging, and a special discussion on the similarities and differences between the Wankel engine, rotary engine, and others. The effects of mufflers on engine performance and their noise reduction capabilities are discussed. 
N3. Noise Radiation from Engine and Drive Train. A. J. Brammer, National Research Council of Canada, Ottawa, K1A OS1, Canada, AND Douglas Muster, University of Houstom, Department of Mechanical Engineering, Houston, Texas 77004.-The rapid pressure rise within the cylinder accompanying spontaneous ignition of the fuel generates the characteristic "knock" produced by diesel engines. Noise is radiated primarily by nonstructural parts of engines such as the oil pan and valve covers, or by the crankcase. In contrast, the rate of change of pressure within the cylinders of sparkignition engines is relatively slow, owing to combustion being nucleated at a point, and results in less radiation, especially at high frequencies. In air-cooled engines, the absence of a water jacket often results in additional radiation from the cylinder cooling fins. Other sources of importance in some engines are those with a mechanical origin such as piston slap or gear meshing. The significance of the contribution of these and other sources to the overall noise produced by vehicles will be indicated.

N4. Aerodynamic Noise from Vehicles. Rorert C. Chanaud, University of Calorada, Department of Civil and Environmental Engineering, Boulder, Colorado 80303, AND Douglas Muster, University of Houston, Department of Mechanical Engineering, Houston, Texas 77004.-The sources of vehicle noise and their importance relative to aerodynamic noise is given. Using the theory of Lighthill, a summary is given of aerodynamic noise theory and experiment. Following a review of the literature on axial and propeller fan noise and on vehicle cooling fan noise, the present state of the art is described, including present noise reduction approaches. The role of aerodynamic noise in internal and external noise spectra is described. An assessment is made of the present state of knowledge and trends in vehicle design. Recommendations are made for future areas of research.

N5. Tire-Road Interaction Noise. IVILliam A. Leasure, Jr., National Bureau of Standards, Washington, D. C. 20234, ERICH K. Bender, Bolt Beranek and Newman Inc., Cambridge, Massachusetts 02138, AND Robert H. Bollinger, Ford Motor Company, Dearborn, Michigan 48128.- The relative importance of tire noise to overall vehicle noise is established. A general discussion is given of the considerations of the tire engineering process and design procedure followed in the manufacture of a tire. The important parameters influencing tire noise are discussed based on the physical data base as it exists today and unknown and contradictory areas are identified. The basic mechanisms of tire-noise generation, although not well defined, are investigated from a theoretical viewpoint. Areas for future research and development are identified based on gaps in the existing physical data base.

N6. Motor Vehicle Noise Bibliography. Douglas Muster, University of Houston, Department of Mechanical Engineering, Houston, Texas 77004. - The manner in which the bibliography can be used is described. Commentaries are made on some of the most important recent publications.

WEDNESDAY, 29 NOVEMBER 1972

El Dorado West, 9:00 A.M.

\title{
Session O. Physical Acoustics II : Ultrasonic Energy and Power Measurements I
}

\author{
JoHN E. MAY, Chairman \\ Bell Laboratories, North Andover, Massachusetts 01845
}

9:00

Introduction

O1. Overview of the Two Special Sessions on Ultrasonic Energy and Power Measurements. W. D. O'Brien, JR., Division of Biological Effects, Bureau of Radiological Health, Food and Drug Administration, Rockville, Maryland 20852.- The applications and uses of ultrasonic energy in the clinical practice of medicine, in industry, and in consumer products are increasing at an accelerated rate. Thus, it is essential, in many cases, that both the manufacturer and user have the knowledge and capability to measure accurately, reproducibly, and easily, both spatially and temporally the intensity $\left(W / \mathrm{cm}^{2}\right)$. power (W) and/or energy (J) of the ultrasonic field. The purpose of the two special sessions on energy and power measurements of ultrasound is to present and discuss the radiation force, optical, calorimetric, and piezoelectric metlods for measuring ultrasunic field parameters in an aqueous medium. Each method will be introduced with a tutorial talk describing the particular physical phenomenon which is being used to examine the ultrasonic field. This will be followed by a panel discussion of specific problem areas, during which time audience interaction will be encouraged. The principal ultrasonic frequency range to be discussed is $100 \mathrm{kHz}$ to $30 \mathrm{MHz}_{\mathrm{z}}(\mathrm{c} \cong 1500 \mathrm{~m} / \mathrm{sec})$. Both continuous and pulsed wave modalities will be considered. As guide-lines, the typical continuous wave intensity range 


\section{$84 \mathrm{TH}$ MEETING ACOUSTICAL SOCIETY OF AMERICA}

is several hundred $\mu \mathrm{W} / \mathrm{cm}^{2}$ to tens of $\mathrm{W} / \mathrm{cm}^{2}$. For pulsed ultrasonic waves, duty cycles as low as $0.05 \%$, pulsewidths as narrow as a couple of cycles and with peak intensity range a few $\mathrm{mW} / \mathrm{cm}^{2}$ to hundreds of $\mathrm{W} / \mathrm{cm}^{2}$ are considered.

\section{Calorimetric Method Tutorial Paper}

02. On The Calorimetric Determination of Ultrasonic Intensities. Adnan Sokollu, Case Western Reserve University, Cleveland, Ohio 44106.- The rate of energy flux in an ultrasonic beam, propagating in a huid medium, may be expressed for a single frequency and longitudinal oscillation by

$$
E_{\phi}=\frac{1}{T} \int_{0}^{T} A p u d t,
$$

where $T$ is the period of oscillations, $A$ the cross section of the beam, $p$ the instantaneous value of pressure oscillations, and $u$ represents particle velocities causing the pressure oscillations. This is the basic equation with which, at the turn of the century, Lord Rayleigh calculated acoustic intensities in gases. Almost half a century later, Langevin was first to measure ultrasonic intensities in liquids by evaluating radiation pressures created by these intensities. A simple theoretical basis to these evaluations was already established two decades prior to his experiments. Further theories were later developed to express more closely the relationship between the radiation pressure and sonic intensities. The complexity of wave propagation phenomena in real media, however, has been preventive in establishing an exact theory, especially in cases of intense or pulsed radiations. Therefore, there is a serious need for alternative methods to determine sonic intensities without resorting to debatable postulations. Only after compilation of reliable data will it be possible, through these methods, to validate or modify the existing theories. The only reliable method, which can avoid debatable assumptions, appears to be the conversion of sonic energy into heat. The heat-conversion method is one of the oldest known to physics in terms of determining a multitude of radional energies. The so-called bolometers or radiometers were the standard instruments used in evaluating heat or light radiation more than a century ago. The purpose of this paper is to review various kinds of calorimetric methods and explore their feasibility to ultrasonic power measurements. Thus we hope to revive and perfect at least one of these classical methods as a primary standard toward the evaluation of newly established methods and theories.

$$
9: 40
$$

\section{Calorimetric Method Panel Discussion}

$\begin{array}{ll}\text { K. L. Churney } & \text { National Bureau of Standards, Washington, D. C. } 20234 \\ \text { F. DunN } & \text { Bioacoustics Research Laboratory, University of Illinois, Urbana, Illinois } 61801 \\ \text { J. B. Pond } & \text { Physics Department, Kingston Polytechnic, Kingston upon Thames, United Kingdom }\end{array}$

$10: 40$

Piezoelectric Method Tutorial Paper

O3. Piezoelectric Transducers for Measurement of Ultrasonic Pressure. D. Berlincourt, Channel Products, Inc., Newbury, Ohio 44065.- The fundamental physical concepts of piezoelectricity are reviewed in some detail with emphasis on application to measurement of ultrasonic pressure. The physical constants of a variety of piezoelectric materials are given and discussed. These data are related to mechanical and electrical matching requirements and some recommendations are given. Various configurations for piezoelectric microphones are discussed with reference to frequency and the properties of the acoustical medium. Linearity of the ferroelectric (piezoelectric) ceramic materials is discussed briefly. Limitations with respect to static pressure and temperature are also considered.

\section{Piezoelectric Method Panel Discussion}
R. J. BOBBER
Underwater Sound Reference Division, Naval Research Laboratory, Orlando, Florida 32806
R. C. EgGLetoN Indiana University School of Medicine and Interscience Research, Institute of the Indianapolis Center for Advanced Research, Indianapolis, Indiana 46202
F. J. F FY
Indiana University School of Medicine and Interscience Research, Institute of the Indianapolis Center for Advanced Research, Indianapolis, Indiana 46202
J. R. REID
Providence Hospital, Seatlle, Washington 98122 


\title{
Session P. Architectural Acoustics II : Application of Correlation Techniques to Measurement in Building Acoustics
}

\author{
Ludwig W. SePmeyer, Chairman
}

\author{
1862 Comstock Avenue, Los Angeles, California 90025
}

All questions will be held until the end of the normal presentations. A freewheeling discussion is then anticipated among the panelists (authors), other experts, and novices in the audience.

\section{Invited Papers (30 minutes)}

P1. Application of Correlation and Fourier Transform Techniques in Architectural and Building Acoustics. J. TICHy, Pennsylvania State University, University Park, Pennsylvania 16802.--This paper presents a historical review, summary, and discussion of existing problems of application of autocorrelation techniques and Fourier transform to measurements in architectural and building acoustics. Existing problems of sound field enclosures will also be discussed.

P2. TM Spectra: A Measure of Diffuseness of Reverberant Sound? Dayid Lubman, D. Lubman \& Associates, 23727 Crosson Drive, Woodland Hills, California 91364.-Diffuseness of reverberant sound is a sine qua non for accurate reverberation room measurement of sound absorption, power, and transmission loss. Most authorities regard it as important for the concert hall as well. And yet, technique for the measurement of diffuseness is not well developed. In this connection, the recently proposed technique of TM (traversing microphone) spectroscopy shows promise. The technique exploits information on diffuseness contained in the spatial correlation function of instantaneous sound pressure. Mathematically, the TM spectrum is the Fourier transform of the spatial correlation function. Physically, it can be interpreted as the power spectrum obtained at the terminals of a small microphone traversing the room at constant speed. For an ideally diffuse field of narrow bandwidth, the TM spectrum is, conveniently, a perfect rectangle. For a less diffuse field, deviations from the rectangular shape will occur. Supporting experimental and analytical results are shown. The limitations and promise of this new technique are discussed. [This work supported by the Office of Naval Research, Code 468.]

P3. A Linear System Approach to Room Acoustics. Norman C. Martin and Bill D. Cook, Cullen College of Engineering, University of Houston, Houston, Texas 77004. - Taking the variables that effect the propagation of sound in a room, such as properties of the room confines, objects in the room, air absorption, etc., and assuming they are linear operators, we can formulate a linear system approach to room acoustics. Given the impulse response of a linear system, it is possible to find by analysis all the properties of the system, whether in the time domain or frequency domain. In relation to any two fixed points in a room, one for source input and the other for received signal pickup, by use of the principles of correlation, a method is available to derive the impulse response of the room. (This is one of several methods, but it is straightforward and successful in practical applications.) It follows that by experimentally determining the impulse response function of the system considered one can obtain in situ values of transmission and reflection coefficients, energy decay rates, and parameters required for prediction of sound levels from multiple coherent sound sources. Use is made of the correlation function a second time to generate standard acoustic quantities from the available impulse response function. [Supported in part by two grants from NASA.]

P4. Combining Fourier and Correlation Techniques to Measure Acoustical Parameters. P. D. ScHomer, Environmental Engineering Branch. Construction Engineering Research Laboratory of the Corps of Engineers, Champaign, Illinois 61820. - The basic means of measuring an impulse response by employing correlation techniques and of extending this response into the frequency domain by utilizing Fourier techniques is reviewed. Particular attention is given to the problems inherent in this form of measurement. These problems include proper utilization and interpretation of the discrete Fourier transform, proper attention to flanking paths, and proper attention to what may be termed the acoustic version of the uncertainty principal. The ramifications of this uncertainty principal to other measurement methods are also considered. Sample results of recent laboratory and in situ transmission loss measurements are presented.

P5. Time Delay Spectrometry. R. C. Hevser, Bioscience and Planetology Section, Jet Propulsion Laboratory, Pasadena, California 01103.- Human experience in the interpretation of sound strongly supports the concept of a time-dependent frequency spectrum. Acoustical testing of architectural structures must therefore be brought into alignment with joint time-frequency considerations to bridge the gap between subjective and objective performance assessment. Time delay spectrometry (TDS) is a method of measurement which directly yields the time-dependent frequency spectrum. This is done by transforming normal acoustic variables to a joint time-frequency coordinate system. 
By this means, acoustical properties that overlap in a pure-time or frequency domain measurement, as for example frequency-dependent multiple reflections, may be separated for analysis. Following separation of the signal of interest, the result is then transformed back to either the time or frequency representation for conventional evaluation, but with the difference that unwanted signals have been suppressed. Time delay spectrometry is a continuous coherent process that operates on a real-time basis allowing evaluation of system parameters in situ. The data obtained includes the complete response, both amplitude and phase, for either time or frequency. Measurements possible with TDS include direct and early sound anechoic spectra in a normally reverberant environment, reverberant energy as a continuous function of frequency and time, and joint time and frequency-dependent sound propagation through partitions.

WEDNESDAy, 29 NOVEMBER 1972

Silver Chimes East, 9:00 A.M.

Session Q. Speech Communication III : Speech Processing and Transmission

Douglas L. Hogan, Chairman

National Security Agency, Fort George G. Meade, Maryland 20755

Contributed Papers (7 minutes)

Q1. Techniques for Processing EMG Signals. D. K. PORT, Haskins Laboratories, 270 Crown Street, New Haven, Connecticut 06510 .-Analysis of electromygraphic activity is potentially a fruitful technique for investigating speech production. However, the EMG signal picked up by bipolar wire electrodes is weak and inherently variable and must be carefully handled. At Haskins Laboratories a computer processing system was developed to analyze simultaneously up to eight EMG channels of data. This system allows for identifying, sampling, and digitally averaging EMG signals for a set of utterances of the same type. Integration of the EMG signals, both in the hardware and software, has been recently revised and its importance to the interpretation of EMG signals will be discussed. The relationship between an averaged EMG signal and the individual utterances in the sample will be discussed in the context of editing and display capabilities. Further, an experiment involving multiple placement of electrodes in both muscles of a bilateral pair has been conducted. Computation of correlation coefficients for utterances across the multiple electrode placements shows that one electrode can provide $E M G$ activity representative of activity in the entire muscle. This is true for both individual utterances and for the average of a set of utterances. [This research was partially supported by the National Institute of Dental Research.]

Q2. Computer-Assisted Measurement System for X-ray Films of the Vocal Tract. P. Mermelstein AND D. $J$. MACLEAN, Bell Laboratories, Murray Hill, New Jersey 07974.Measurements on sagittal $x$ rays of the vocal tract may be made directly in terms of the variables of an articulatory model by superimposing a computer-generated vocal-tract outline onto the $\mathrm{x}$-ray image. Previous methods require an operator to trace a pen or pointer coupled to a recording device along the outlines of the projected image. The new system uses two video signals, one derived from a television camera scanning the projected $x$-ray image, the second from data in a computer display buffer. The computer display is modified through adjustment of control potentiometers assigned to the model variables. Convergence to a match between the two images is found to be substantially quicker and easier task than manually tracing the image outline.
Q3. Intermodulation Distortion Produced by Center Clipping. O. M. M. Mitchell and G. H. Yates, Bell Telephone Laboratories, Incorporated, Holmdel, New Jersey 07733.-Recently, center clipping has been used in a number of speech processing systems [O. M. M. Mitchell and D. A. Berkley, J. Acoust. Soc. Amer. 47, 84 (1970); O. M. M. Mitchell and D. A. Berkley, Bell System Tech. J. 50, 1619-1630 (1971)]. In this paper, we apply Bennett's double Fourier series technique [H. E. Rowe, Signals and Noise in Communication Systems (Van Nostrand, Princeton, N. J., 1965)] to obtain integral expressions for the distortion products of two center clipping transfer functions for the case of two simultaneous sinusoidal inputs at arbitrary frequencies. Results of numerical solutions are presented for six clipping levels and six values of relative amplitude of the two sinusoidal inputs.

Q4. Low Bit-Rate Coding from the Autocorrelation Method of Linear Prediction. J. D. MARKel AND A. H. GRAY, JR., Speech Communications Research Laboratory, Inc. (SCRL), Santa Barbara, California 93101.- The term "autocorrelation method" encompasses the inverse filter, maximum likelihood, and PARCOR method. This method has a significant computational advantage over Atal's covariance method since the analysis does not require polynominal root testing programs and associated modifications, and is thus much easier to implement. Informal listening tests indicate that for low bitrate coding, the resulting synthetic speech is very similar for both methods with fixed frame analysis. Directly from the autocorrelation equation solution, the filter parameters, $k$ parameters (also referred to as reflection coefficients and PARCOR parameters) are obtained, along with the synthesizer gain parameter. Low bit-rate coding based upon the $k$ parameters has been obtained by performing the following steps: (1) prefilter sharply at the half-sampling frequency; (2) prewhiten and Hamm before analysis; (3) transform the parameter values to $[-0.7,0.7] ;(4)$ quantize with rounding; and (5) interpolate synthesis parameters pitch synchronously. These operations are all very fast and easily implemented. Good quality synthesis over a $3200-\mathrm{Hz}$ input bandwidth is obtainable with $\left\{k_{i}\right.$ BITS, $\left.i=0,1, \cdots, 7\right\}=\{6,6,3, \cdots, 3\}$. Examples coded at 2100 and 1400 bits/sec $(50$-and $33-\mathrm{Hz}$ frame rates, respectively) are presented. 
Q5. The Application of Dither to the Quantization of Speech Signals. L. R. RABiner, N. S. Jayant, and J. A. Johnson, Bell Telephone Laboratories, Murray Hill, New Jersey 07974.By adding a pseudorandom "dither" noise to a signal $X$ that is to be quantized, and by subtracting an identical noise sequence Irom the quantizer output, it is possible to break up undesirable signal-dependent patterns in the quantization error sequence, without increasing the variance of the error $E$. The idea has been widely discussed in the context of picture coding, and it is the purpose of this talk to demonstrate application of the technique to the quantization of speech signals. Computer simulations have shown how the use of dither whitens the quantization error sequence in PCM encoding, and renders it more acceptable than signal-correlated errors of equal variance. We demonstrate, for conditions of dither and no dither, typical speech recordings, illustrative error waveforms, and data on signal-to-error correlation $C$; and indicate how the advantage of dithering increases monotonically with crudeness of signal quantization and becomes significant when the number of bits per sample is less than about six. An experimental evaluation of the perceptual effects of dither on speech has shown (a) strong preferences for dithered speech over straight PCM encoding at identical bit rates; (b) an increase in word intelligibility for dithered speech over PCM speech when 4-6 bits/sample were used; and (c) a decrease in word intelligibility for dithered speech over PCM speech when 2-3 bits/sample were used. The above results imply that dithering is most advantageous for speech quantized in the 4-6 bits/sample range.

Q6. Adaptive Quantization in Differential PCM Coders. P. Cummiskey and J. L. FlanaGan, Bell Laboratories, Murray Hill, New Jersey 07974.-A differential PCM coder is described in which the quantizer is automatically adapted to the error variance. The transmitted bit stream is examined by digital logic which in turn adjusts the quantizer step size to reduce slope overload and granular distortion. For a given bit rate, increased quality over PC.M and DPCM coding results. The receiver incorporates adaptation logic identical to that of the transmitter, and reconstructs a signal estimate similar to that made by the transmitter. Digital hardware implementation of the coder is described, and transmission performance at rates of 18,24 , and 32 kilobits/sec is demonstrated.

Q7. Some Baseband Speech Processing Experiments. D. C. Coulter, Communications Sciences Division, Naval Research Laboratory, Washington, D. C. 20390.-Work with a voiceexcited formant vocoder has led to experiments on several other methods of processing speech. These methods make use of the baseband and some of the same types of information which are normally derived by a formant vocoder, such as front-back vowel detection and voice-voiceless discrimination, but applied in a manner different from a normal formant vocoder. The systems so far tested are characterized by transmission of the baseband in unprocessed form and the regeneration of higher frequency information by deriving it from the baseband or simple parameters. While these systems do not yield high quality speech, the intelligibility may be high enough for certain limited applications. Tape recordings of these systems will be presented.

Q8. Signal Processing Techniques Based upon a Wave Packet Representation. D. L. RET2, Computer Systems Laboratory, University of California, Santa Barbara, California.-The method of performing a time-domain approximation of the speech wave in terms of a linear combination of Gaussian wave packets is evaluated as a signal processing tool. This set of functions is used because of their ease of extraction from the original signal and their versatility. The parameters defining the set of functions are determined from the times and magnitudes of extrema in the band-limited signal. Because the wave packets used to represent the signal are analytically transformable to the frequency domain, various characteristics, such as the Fourier spectrum and the autocorrelation function, may be evaluated directly from the parameters describing the wave packets. The economics and accuracy of this technique are discussed.

Q9. Helium Speech Unscramblers: A Critical Review. H. B. Rothman, H. Hollien, and P. Hollien, Communication Sciences Laboratory, University of Florida, Gainsville, Florida 32601.-Man's attempt to probe and utilize "inner space" have, to a large degree, been hampered by inadequate voice communication. One of the specific sources of speech degradation is the use of helium as an inert breathing gas in the deep and/or saturation, diving situation. In turn, electronic devices have been developed which are designed to improve speech intelligibility distorted by the effects of high ambient pressures and $\mathrm{HeO}_{2}$ breathing mixtures. In order to evaluate the effectiveness of these $\mathrm{HeO}_{2}$ "unscramblers," the Communication Sciences Laboratory at the University of Florida has undertaken a four-part project: (1) to evaluate available unscramblers on line; (2) to test them off line; (3) to develop a standardized test for evaluating all types of unscramblers; and (4) to establish guidelines for the development of optimum equipment of this type. The present paper will discuss the following: (1) techniques for helium speech unscrambling; (2) protocols necessary to conduct system evaluation properly; (3) development of the standardized off-line test for the evaluation of all types of unscramblers; and (4) results of several on-line and off-line evaluations. Finally, a discussion of the strengths and weaknesses of current approaches to $\mathrm{HeO}_{2}$ speech processing, and recommendations for the improvement of these devices will be presented.

Q10. Evaluation of Speech Processing Innovations to Improve Voice Quality and Intelligibility in Narrow-Band Voice Communication. Caldwell P. Smith, Chicopee Row, Groton, Massachusetts 01450.-Using an experimental hardware channel vocoder as a test bed, a systematic series of tests including formal and informal listener evaluations were conducted to evaluate the merits of several speech processing innovations towards maximizing speech quality and intelligibility. A superior combination was found in a technique that enhances spectral peaks prior to digitization [a refinement of an old concept: C. P. Smith, J. Acoust. Soc. Amer. 23, 446-451 (1951)] together with a synthesis excitation arrangement using oscillators synchronously phase reset with each pitch excitation pulse [C. P. Smith, J. Acoust. Soc. Amer. 37. 170-171 (1965)], with added refinements for enhancing phase coherence in onsets of pitch epochs in voiced sounds. Although pitch extraction and coding were relatively crude (300 bits/ sec pitch data rate, and occasional pitch errors), this combination yielded superior results such that quality and intelligibility at 2400 bits/sec, depending on the speaker, either exceeded or was fully comparable to that of a good 9600 bits/sec voice-excited vocoder. These findings suggest a potential for refining $2400 \mathrm{bits} / \mathrm{sec}$ hardware channel vocoders to derive superior performance/cost tradeoffs relative to any other existing technology. [This work was performed in the now-terminated Digital Speech Processing Branch of Air Force Cambridge Research Laboratories.]

Q11. Zero Quefrency in Speech Analysis. John MakhouL, Boll Beranek and Newman Inc., Cambridge, Massachusetts 02138. - The zero component of the cepstrum (transform of the $\log$ spectrum) is also known as the zero quefrency $c_{0} . c_{0}$ contains information about $R_{0}$ (the total energy in the signal), as well as the shape of the signal power spectrum. 
The energy information can be removed by subtracting $\log R_{0}$ from $c_{0}$. If that difference is exponentiated, one obtains a normalized parameter $V=e^{c_{0}} / R_{0}$. $V$ can be interpreted as the ratio of the geometric mean to the arithmetic mean of the spectrum. For any spectrum, it can be shown that $0 \leq V \leq 1$. The value of $V$ depends only on the spectral shape. By taking advantaye of the fact that most voiced sounds have the energy concentrated at low frequencies, while most unvoiced sounds have the energy relatively spread out across the spectrum, the parameter $V$ can be used as a voicing detector. $A$ detailed analysis of the behavior of $V$ as a lunction of different spectral shapes can be found in Makhoul and IFolf [BBN Rep. No. 2304 (1972)]. Conditions under which $V$ can be used as a voicing detector will be discussed.

Q12. Speech Quality of Computer-Simulated Voice-Switched amplifiers. Herman R. Silbiger, Richard E. Cullingford, AND Linda Pierce, Bell Laboratories, Holmdel, New Jersey 07733. - Voice-switched amplification can be used in telephone circuits to provide additional amplification in the direction of transmission only when speech is present and thus increase the apparent $\mathrm{S} / \mathrm{N}$ ratio. The effect on speech quality is a function of operating characteristics such as attack time, release time, switching thresholds, and amount of switched amplification. To evaluate the detectability and speech quality with such amplifiers, subjective tests were conducted in which computer simulation was used to provide convenient and accurate control of the operating characteristics. In the detection tests, the subjects determined whether they could hear the operation of the voice-switched amplifier, using a modified double staircase method with variable step size. The results were used to map out a region in which the operation of the switched-gain device was not detectable. A category judgment procedure using a 5-point scale was used for the transmission quality listening tests in the previously determined detectability region. Decay times of 25, 100, and $300 \mathrm{msec}$ were presented with switched gains of $0,10,15$, and $20 \mathrm{~dB}$, over circuits with $\mathrm{S} / \mathrm{N}$ ratios of 10 and $30 \mathrm{~dB}$. Results indicated that for a $10-\mathrm{dB} \mathrm{S} / \mathrm{N}$ ratio, the effects of amount of gain switched and decay times were both significant and had a strong interaction. For a $30-d B S / N$ ratio, only the effect of decay times was significant. The 10-, 15-, or 20-dB switched gain conditions were always preferred over the $0-d B$ condition.

Q13. Speech Dereverberation. J. B. Allen, Bell Telephone Laboratories, Holmdel, New Jersey 07733.-A method for dereverberating speech based on linear prediction is developed in detail. By properly analyzing and resynthesizing speech that has been reverberated by a real room, it is possible to remove most of the reverberation. The algorithm will be described and tapes will be played.

WEDNESDAy, 29 NOVEMBER 1972

El Dorado East, 9:00. A.M.

Session R. Underwater Acoustics II : Propagation II

Peter Tatro, Chairman

U. S. Naval Research Laboratory, Washington, D. C.

\section{Contributed Papers (12 minutes)}

R1. Observation of Frequency-Dispersive High-Order Modes in Deep Sound Channels. Rorert P. Porter, Woods Hole Oceanographic Institution, Woods Hole, Massachusetts 02543.Large time spread of signal arrivals is characteristic of deep, sound-channel propagation of wide-band signals and is normally explained by ray acoustics; high-order mode theory predicts time spreading of the arrival and, in addition, predicts dispersion of the spectrum. For a given sound-velocity profile and transmitter-receiver geometry, only a few high-order modes are transmitted and received; it is possible to detect individual dispersion curves if certain time bandwidth requirements on the receiver are satisfied. Dispersed high-order modes have been observed at frequencies as high as $300 \mathrm{~Hz}$ and at ranges of $600 \mathrm{~km}$ for shots detonated and received on the axis of a SOFAR channel located in the western Mediterranean Sea. Spectrum analysis of shot records has revealed up to five identifiable group-velocity profiles that are accurately predicted by applying WKB high-order mode theory to sound velocity data. Group-velocity profiles for North Atlantic sound-velocity data typical of Bermuda water, MidAtlantic Ridge water, and Mlediterranean overflow water are presented. These dispersion curves show the effects of multiple sound-velocity minima and near parabolic sound-velocity profiles on pulse structure. [This work was supported by the U, S. Navy Office of Naval Research.]
R2. Behavior of Modified Ray Theory. J. A. DAvis, Woods Hole Oceanographic Institution, Woods Hole, Massachusetts 02543.-Both modified ray theory [E. L. Murphy, J. Acoust. Soc. Amer. 47, 899-908 (1970) and J. A. Davis, J. Acoust. Soc. Amer. 52, $137(1972)]$ and ordinary ray theory can be obtained from the method of stationary phase. Use of a WKB approximation leads to two points of stationary phase and the result is ray theory. Use of a generalized WKB approximation leads to an infinite number of stationary phase points and the result of using the first two is modified ray theory. The degree to which modified ray theory accurately includes frequency dependent diffraction effects depends on the contribution to the field by the remaining points of stationary phase. In sound propagation problems where there is a shadow region, the ray theory field may or may not contain a caustic, whereas the modified ray theory field always contains a caustic and in some cases a cusped or higher-order caustic. For ranges much less than the shadow boundary range the modified ray theory field approaches the ray theory field. For ranges much greater than the shadow range, the modified ray theory field (suitably extended to take the caustic into account) approaches either (1) the ray theory field (also extended), or (2) the field due to the first mode, depending on whether or not the problem parameters are such that a ray theory caustic is present. For ranges in the vicinity of the shadow range, the behavior can be very complicated. 
R3. Calculating the Wave Field Diffracted at the Boundary of a Shadow Zone. A. A. Hunimac, $S R A$, Inc., Rockville, Maryland 20854.-The conventional ray theory of ten generates shadow zones. Previously the author has given the density of simple sources and dipoles whose radiation accounts for the diffraction at shadow boundary. For a stratified fluid, the boundary is a contiguum of parallel rings starting with one tangent to the shielding plane. Three classes of rings are treated. Those nearly the same distance from the obstructing plane as is the field point are treated by an approximate wave theory. Their contribution is insignificant except for points near the boundary. Those beyond contribute insignifcantly. Those nearer are treated by an approximate ray theory. Most of this diffraction comes from the rings near the tangent ring. For a field point inside the boundary, ray rings all the way to the tangent ring contribute, and the diffraction drops off slowly with distance from the boundary. As the point moves into the shadow zone, more rings near the tangent plane fail to contribute and the field drops off rapidly. The degree of accuracy of other types of calculations (e.g., Rytov, phase integral) shall be discussed.

R4. Ray and Wave Solutions for Bottom Reflection for Linear Sediment Layers. HALCYoN E. MorRIs, Naval Undersea Center, San Diego, California 92132.-Agreement between experimental and calculated values of bottom loss have been shown in earlier work [H. E. Morris, J. Acoust. Soc. Amer. 48, 1198-1202 (1970)]. The mathematical treatment involves solution of the wave equation for a plane wave incident on a set of absorbing sediment layers with velocity gradients. In order to clarify the physical mechanisms that produce the total reflected sound beam, a ray theory solution is presented in which the sound that reflects for the water-sediment interface can be separated from sound that is refracted in the sediment layers. Curves showing the contributions of reflected arrival and various orders of refracted arrivals are presented for several cases of interest.

R5. Generating Parameterless Examples of Ray Acoustics. D. H. Woon, New London Laboratory, Naval Underwater Systems Center, New London, Connecticut 06320.-It is well known that in an unbounded medium having uniform speed, travel time is equal to the distance from a sound source and intensity is equal to its reciprocal. No ray parameters appear in the statement of these results. In general, ray parameters are a necessary but undesirable by-product of the standard technique for finding intensities. A new technique generates many parameterless examples by applying three principles to known parameterless examples such as uniform media. In one principle, limited to two-dimensional media, conformal mapping generates a new example having different waveIronts. For example, $c=\sin x$ is generated from $c=x$ by the conformal mapping defined by $f(x+i y)=\tan (x / 2+i y / 2)$. A second principle generates new examples having the same wavelronts. For example, $c=x$ and $c=1+x^{2}+y^{2}$ have the same wavefronts when a point source is located at $(1,0)$. A third principle relates symmetric examples in two dimensions to cylindrically symmetric examples in three dimensions.

R6. Eramples of Parameterless Intensity Contours. D. $\mathrm{H}$. Wood AND ANdrew A. Lesick, New London Laboratory, Naval Underwater Systems Center, New London, Connecticut 06320.-Intensity contours for several unbounded inhomogeneous media are found and graphed. Both layered and nonlayered examples are included. Intensity contours are taken from parameterless examples of ray acoustics. That is, they are found without using rays, ray equations, or ray parameters. These examples are natural test cases for numerical procedures that also produce intensity contours. Examples of intensity contours generated by parameterless and numerical methods are compared.

R7. Sound Propagation along a Sloping Bottom in Shallow Water. Louis A. KING and D. G. Browning, Nere London Laboratory, Naval Underwater Systems Center, New London, Connecticut 06320.- One of a series of sound propagation experiments recently conducted by the Naval Underwater Systems Center in the Gulf of Aaen took place across a basin with a sloping bottom. Three receiving hydrophones were located near one edge in $60 \mathrm{~m}$ of water. Results of this experiment in which bomb shots were used were compared with both a propagation model based on ray theory by $J$. P. Jones and P. W. Smith, Jr. (Bolt Beranek and Newman TM 84 of 7 July 1971) and a model based on normal mode theory. Since the received signal appeared as an impulse followed by a reverberant portion, each with different spatial properties, the two segments were treated separately.

R8. Sound Pressure in Water from Source in Air and Vice Versa. Rogert W. Young, Naval Undersea Center, San Diego, California 92132.- Sound pressure level $L_{p}$ at a depth $d$, in water, due to a point source in air at a distance $h$ above the water surface, may be calculated from $L_{p}=\left[L_{a}-7+20 \log \right.$ $(\cos \theta)]-20 \log \left(r / r_{s}\right)$, where $L_{a}$, the source level, is the SPL in air at distance $r_{a}$ from the source; $r$ is the straight-line distance to the receiving hydrophone from a virtual sound source situated under the actual source at height $h^{\prime}=\left(c_{1} / c_{2}\right)$ $X h$ above the surface; $c_{1}$ and $c_{2}$ are the respective speeds of sound in air and water; $\theta$ is the angle between the vertical and the line of length $r$. Comparisons with various published results obtained by more sophisticated ray-theory show agreement within $1 \mathrm{~dB}$, except at shallow depths and far sidewise from the sound source; agreement within $2 \mathrm{~dB}$ is found for new experimental data here presented for sound bursts of frequency 500,1000 , and $2000 \mathrm{~Hz}$, and $h=3.5 \mathrm{~m}$. For sound originating in water at depth $d$ below the surface, the SPL received in air is to be calculated from $L_{p}=L_{q}-52$ $+40 \log (\cos \theta)-20 \log \left(r / r_{a}\right)$, where $L_{\text {, }}$ is the source level, at $r_{b}$, in the water. Experimental data obtained with a sound source in the water at depth $d=5.6 \mathrm{~m}$, and frequency 500,1000 , or $2000 \mathrm{~Hz}$, are in agreement with this equation, mostly within $2 \mathrm{~dB}$, for receiving positions in air 1 or $2 \mathrm{~m}$ above the water and offsets as great as $3 \mathrm{~m}$.

R9. Utilization of a Transmitting Array Matched to Individual Modes. W. G. Kanabis, New London Laboratory, Naval Underwater Systems Centers, New London, Connecticut 06320.A vertical transmitting array was implanted in Block Island Sound in November 1970. A theoretical study was undertaken to determine the optimum utilization of such an array to excite individual normal modes. The results of this study are described. Amplitude and phase matching, the two basic methods to emphasize individual modes, are outlined. In matching to amplitude, either a course or more exact fit to the mode amplitude may be utilized. These three approaches to emphasize individual modes were considered. Simple cases of these approaches were evaluated for the physical characteristics of the implanted array using a computer program. BIFI velocity profiles typical for summer and winter were used. Propagation loss was calculated for individual modes when the array was matched in turn to modes 1-4 for each profile and mode. In this manner, the relative signal levels corresponding to each approach were obtained and their effectiveness evaluated.

R10. Estimates for the Reflection of Acoustic Waves Propagating through a Layered Thermocline. Roy C. ELswick New London Laboratory, Naval Underwater Systems Center New London, Connecticut 06320.-The oceanic thermocline contains a weak small-scale steplike structure (relative to 
scales of the overall thermocline) capable of producing at any given layer a weak reflection of an acoustic wave. By means of a two-variable asymptotic expansion technique, the cumulative effect of a series of such weak reflections is accounted for, indicating (similar to the Bremmer series for electromagnetic waves in a slowly varying and thus single-scaled stratified medium) that each higher-order contribution corresponds to a specific number of internal reflections. Simple first-order estimates can be derived for the loss due to an acoustic wave propagating through a stepped thermocline structure. Such order of magnitude estimates indicate that the cumulative effect of the weak reflections is indeed a plausible explanation for the apparent loss of acoustic energy across the thermocline region.

R11. The Nature of Sound Fluctuation in the Upper Ocean. I. J. A. Neubert, Naval Undersea Center, Pasadena Laboratory, Pasadena, California 91107.-In this critique of sound propagation studies in turbulent fluids, the interpretations and limitations of some well known scattering theories and experiments are brought into question. First, some earlier theories of sound propagation in the turbulent ocean are reviewed. Second, some of the more recent developments in the analysis of sound propagation in turbulent fluids are presented. Finally, the earlier results and the contemporary accomplishments are compared in the single-scattering and one-dimen- sional cases. The more important revelations and confirmations, based on Part I, are reserved for Part II where it is shown, on the basis of turbulent scaling, that the scattering theories of Bergmann, of Mintzer, and of Potter and Murphy deserve closer scrutiny and that the Stone and Mintzer experiments were misinterpreted.

R12. The Nature of Sound Fluctuation in the Upper Ocean. II. J. A. Neubert, Naval Undersea Center, Pasadena Laboratory, Pasadena, California 91107.-- Having reviewed and critiqued the nature of sound fluctuation in turbulent media and the upper ocean in Part $\mathrm{I}$, it is now shown, on the basis of turbulent scaling, that the scattering theories of Bergmann, of Mintzer, and of Potter and Murphy are discrete-scattering, and not continuous-scattering, theories when Gaussian refractive-fluctuation correlations are imposed. Furthermore, turbulent scaling and the experimentally observed turbulent microstructure strongly suggest that the scattering mechanism in the Stone and Mintzer experiments is not the turbulence, but is more likely impurities (such as the ubiquitous, minute air bubbles which arise due to thermal degassing) that are shuffled about in a random manner by the turbulent fluid. It is strongly suggested that this is of ten the nature of sound fluctuation in the upper ocean. Finally, more effective methods of analyzing sound fluctuation in the real ocean are presented.

WEDNESDAY, 29 November 1972

Burgundy EAst, 2:00 P.M.

\title{
Session S. Physiological Acoustics II : Models and Cochlear Electrophysiology
}

\author{
E. A. Peterson, Chairman
}

Division of Auditory Research, University of Miami School of Medicine, Miami, Florida 33152

\section{Contributed Papers (12 minutes)}

S1. A Nonlinear Model for Basilar Membrane Motion. Duck ON Kim, Charles E. Molnar, and Russell R. PfeIffer, Washington University, St. Louis, Missouri 63130.A phenomenological model for basilar membrane motion is presented. The model consists of 10 cascaded elements defined by the nonlinear differential equations

$$
x_{i}(t)+2 D_{i}\left[1+\eta \dot{x}_{1}^{2}(t)\right] \dot{x}_{i}(t)+\omega_{0}^{2} x_{i}(t)=C x_{i-1}(t)
$$

for $i=1,2, \cdots, 10$, where $x_{0}(t)$ is the input (stapes displacement) and $x_{10}(t)$ is the output (displacement of a point on the basilar membrane). The model demonstrates a close relationship among seemingly disjoint. nonlinear phenomena of the peripheral auditory system by showing the following response characteristics: (1) saturation of the output amplitude, decrease of $Q$, and decrease of the maximally responsive frequency with increase of input amplitude; (2) "amplitude" and "temporal" nonlinearities in response to combination clicks; (3) generation of combination tone $2 f_{1}-f_{2}$ in response to inputs of two equal-amplitude tones $\left(f_{1}<f_{2}\right)$; (4) two-tone suppression for frequencies of the suppressing tone above the characteristic frequency; (5) change of the phase angle of the output with increasing amplitude of sinusoidal input; and (6) change of click response pattern with increasing click amplitude. The characteristic (1) has already been observed directly in basilar membrane motion. We suggest that the characteristics (2)-(6), which are similar to nonlinear phenomena observed in spike-discharge patterns of single cochlear nerve fibers, may actually be properties of basilar membrane motion.

S2. Simulation of a Nonlinear Model of the Basilar Membrane on a Digital Computer. J. L. HALL, Bell Laboratories, Murray Hill. New Jersey 07974.- The generation and propagation of distortion products in a nonlinear model of the basilar membrane was studied. The basilar membrane was modeled as a 175-section transmission line, with hydromechanical coupling between sections. A version of this transmission line developed by Schroeder provides good agreement with Mössbauer effect and capacitive probe measurements of motion of the basilar membrane. The model was simulated on a Honeywell DDP516 computer as a sampled-data system represented in terms of the bilinear $z$ transform. Nonlinearities were introduced by making the compliance of the basilar membrane depend on its displacement. Both odd- and even-order distortion products have been investigated. Distortion products $f_{2}-f_{1}$ and $2 f_{1}-f_{2} \quad\left(f_{2}>f_{1}\right)$ are generated predominantly at the $f_{2}$ place and propagate to their own characteristic place, and so can be equated to tones of frequency $f_{2}-f_{1}$ and $2 f_{1}-f_{2}$ applied at the input. This is not the case for distortion product $2 f_{2}-f_{1}$. 
S3. On a Dynamical Mechanical Model of a Bat's Middle Ear. Gerald Fleischer, Department of Otolaryngology, University of Miami, School of Medicine; Miami, Florida 33152. - An enlarged model of a bat's middle ear (diameter of the ear drum is $20 \mathrm{~cm}$ ) was made out of steel, lead, and aluminum. The model allows the variation of the tension of the tympanic membrane, the fixation of the malleus, the stiff ness of both articulations of the ossicular chain, and the rigidity of annular ligament. Furthermore insertion and force of both middle-ear muscles can be altered, as well as size and location of the orbicular apophyse. (The latter is an additional bulky mass of bone at the malleus, present in bats, in some insectivores, and in some rodents.) Frequency characteristic and the mode of vibration of the ossicular chain will be shown after stimulation with sound as well as with a punctually applied mechanical movement. Measuring transducers are piezoelectric elements. The influence of some of the above mentioned parameters on the transmission of vibration will be demonstrated. Finally the model will be stimulated with music and speech by means of an acoustical and a punctually applied mechanical analog signal. The recording from the base of the stirrup will be presented to the auditorium as playback of a tape recording.

S4. The Temporal Relationship Between Motion of the Basilar Membrane and Initiation of Nerve Impulses in the Auditory Nerve Fibers. T. Konishi* and D. W. Nielsen, Communication Sciences Laboratory, Department of Speech, University of Florida, Gainesville, Florida 32601.-It has been suggested that the initiation of nerve impulses in the auditory nerve fibers is associated with the deformation of the basilar membrane toward the scala vestibuli. In order to verify this possibility, the temporal patterns of nerve impulses of the auditory nerve fibers were examined in the guinea pig, when the basilar membrane was statistically displaced. This displacement was produced by driving the round window membrane with an electromechanical transducer, after the helicotrema had been plugged with bone wax. The electrical signal to the transducer was a trapezoid wave. The hair-cell responses to this trapezoid motion, recorded from the scala media, demonstrated a unidirectional displacement of the basilar membrane. Our results indicate that the displacement of the basilar membrane toward the scala tympani causes the tonic excitation in about $45 \%$ of the primary neurons and the tonic inhibition in 3\%. Displacement in opposite direction shows the reverse effects. About $20 \%$ of our total data showed responses during rising and/or falling period of the trapezoid stimuli. These responses were observed in about $50 \%$ of the fibers with middle or low best frequencies. [Supported by NINDS Grant.]

* Also Division of Otolaryngology and Department of Physiology, University of Florida.

S5. Local Potentials Recorded from the Tunnel of Corti. Merle Lawrence, Alfred L. Nuttall, and Maxine ClaPPER, Kresge Hearing Research Institule, University of Michigan Medical School, Ann Arbor, Michigan 48104.-Microelectrodes are inserted into the cells and fluid spaces of the organ of Corti under direct visual control monitored by closed-circuit television. Local dc resting potentials, tissueelectrode impedance, and ac potentials resulting from fluid vibrations applied by two methods are simultaneously recorded. Vibrations are applied either directly to the stapes or to the basilar membrane in the area of electrode insertion. The ac response recorded from the polarized cells and spaces of the organ of Corti are different depending upon whether the ac is locally or remotely generated. The magnitude and distribution of dc resting potentials are greatly dependent upon the local physiological condition of the exposed organ of Corti. Of most importance are an intact capillary system in the local area and good general physiological condition of the animal. Location of electrode tip is determined visually followed by microscopic examination of surface preparations.

S6. The Origin of $\mathrm{N}_{2}$ of the Cochlear Whole-Nerve Action Potential. J. E. Pugh, Jr., D. J. Anderson, P. A. Burcio, AND M. R. Honwitz, Kresge Hearing Research Institule, University of Michigan Medical School, Ann Arbor, Michigan 48104.-Several studies exist concerning the nature of the cochlear whole-nerve responses to acoustic transients. All focus on the properties of the first negative deflection $N_{1}$, but none offers a satisfactory explanation for $N_{2}$. A number of lines of evidence are here presented to support the concept of separate loci of generation of $N_{1}$ and $N_{2}$ : (1) Changes in relative position of recording electrodes produce differential changes in amplitude of $N_{1}$ and $N_{2} ;$ (2) paired-click studies permit electrical summation of an $N_{2}$ response with a succeeding $N_{1}$ response; (3) high-frequency noise masking attenuates $N_{1}$ to a greater degree than it does $N_{2} ;(4)$ certain intensities of low-frequency masking attenuate $N_{2}$ to a greater degree than they do $N_{1}$; (5) a narrow-band noise-induced temporary threshold shift between 6 and $14 \mathrm{kHz}$, in behaviorally trained monkeys with chronic electrical implants, causes a marked reduction in $N_{1}$ amplitude but no significant change in $N_{2}$; (6) selective cooling of the apex of the guinea pig cochlea results in a relatively greater reduction in $N_{2}$ than in $N_{1}$. These and other lines of evidence suggest that the $N_{2}$ potential may be arising from a more apical region of the cochlea than $N_{1}$. [This work was supported by the John A. Hartford Foundation and by NIH.]

S7. Comparison of Effects of Biasing the Cochlear Partition with Electrical Current and Sound Stimulation on the DIF SP Responses. John D. Durrant and Peter Dallos, Auditory Research Laboratory, Northwestern University, Evanston, Illinois 60201.-Summating potential responses were measured utilizing the differential recording technique. Two experimental designs were incorporated, one in which the cochlear partition was electrically biased with direct current and the other in which the movement of the partition was "biased" with a low-frequency sound stimulus. The DIF" and $\mathrm{DIF}^{+}$components were of prime interest. It was found that dc polarization and the mechanical bias have strikingly similar effects on the SP under certain conditions. The SP can be reversibly augmented or depressed according to the direction of current flow or direction of the biasing displacement, and, furthermore, the two DIF components exhibit change in the same absolute direction. A decrease in the DIFand an increase in the $\mathrm{DIF}^{+}$, and vice versa, is produced by a given biasing stimulus. It is surmised that the net biasing effect is such that when the scala media potential is caused to increase (directly by current or indirectly by deformation of the cochlear partition), the DIF SP responses are made more negative, and more positive when the scala media potential decreases. The similarity of the changes in these SP responses observed under the two forms of biasing suggests that they act on essentially the same distortion process, which appears to be primarily of electromechanical origin. [Supported by grants from the National Institute of Neurological Diseases and Stroke.]

S8. Ethacrynic Acid Ototoxicity, Potentiation by Kananycin. Jiri Prazma, J. P. Browder, and N. D. Fischer, Department of Surgery, University of North Carolina School of Medicine, Chapel Hill, North Carolina 27514.-Endocohlear potentials (EP), cochlear microphonics (CM), and action potentials 


\section{TH MEETING A ACOSTICAL SOCIETY OF AMERICA}

(AP) have been recorded from guinea pig cochleas in animals anesthetized by Nembutal. The experimental animals were divided into four groups for two acute experiments and two chronic experiments. In the acute experiments, groups 1 and 2 received 50 or $100 \mathrm{mg} / \mathrm{kg}$ of Kanamycin and $10 \mathrm{mg} / \mathrm{kg}$ Ethacrynic acid, both drugs twice at $1 \mathrm{~h}$ intervals. In the chronic experiments, groups 3 and 4 received 50 or $100 \mathrm{mg} / \mathrm{kg} /$ day of Kanamycin for 14 days, and on the last day, after evaluation of base line potentials, received $10 \mathrm{mg} / \mathrm{kg}$ of Ethacrynic acid only. Each drug administered alone was not ototoxic or was only mildly ototoxic; however, combination treatment by both drugs potentiate the ototoxic effect. Detailed findings on cochlear potentials will be described. [This research was supported by $\mathrm{NIH}$ grants.]

S9. Nature of the Cochlear Changes Induced by Ethacrynic acid. S. K. Bosher, C. Smith, and R. L. Warren, Ferens Institute, Middlesex Hospital Medical School, London WIP $5 F D$.- A comprehensive study of the effects of ethacrynic acid upon the cochlea in a single species is being undertaken to determine more exactly the nature of its toxic action. In the rat, the drug produces rapid replacement of the endocochlear potential by a negative potential and, while recovery is initially rapid, its rate decreases considerably at $30 \mathrm{~min}$. The endolymphatic chemical composition remains unchanged, however, until $35 \mathrm{~min}$, when increase in the sodium and decrease in the potassium concentrations occurs. The drug is consequently considered to cause transient inhibition of the strial enzymes, followed by alterations in membrane permeability. The ultrastructural strial anomalies are associated with and thought to arise from the permeability changes. The microphonic responses and morphological examination provide evidence of independent hair cell involvement, but its nature and relationship to the endolymphatic changes is uncertain. In addition, the findings strongly support the concept that the endocochlear potential has two components, a positive secretion potential and a negative potassium diffusion potential.

S10. Whole-Nerve Action Potentials as an Index of Severity of Cochlear Injury in Chinchilla. D. H. Eldoredge, Central Institute for the Deaf, St. Louis, Missouri 63110.-The $N_{1}$ peak voltage of the AP response to a wide-band click is proving to be a sensitive index to injuries following exposures to noise. Over a wide range of injury, changes in magnitude and sensitivity of $N_{1}$ responses correctly rank ears with respect to amount of loss of hair cells. With loss of 100-300 hair cells, the voltages are one to two standard deviations below normal means at all levels of the input-output function. The voltages are down two to three standard deviations with 300-800 missing cells. With greater losses the input-output functions are also shifted to a lower sensitivity. A small response can still be elicited $70 \mathrm{~dB}$ above normal visual detection levels when an estimated 6800 out of $9100(75 \%)$ hair cells are missing. Differential masking of the $N_{1}$ responses with selected bands of noise provides additional information concerning the locations of the injuries.

S11. A Thin-Film Intracochlear Multimicroelectrode. MARTIN SonN, Raytheon Company, Portsmouth, Rhode Island 02871, and Department of Otolaryngology, Boston University Medical Center, Boston, Massachusetts 02118.-A Hexible, intracochlear multimicroelectrode array is being developed for differential electrical stimulation of the cochlear nerve in human sensory deafness [M. Sonn, Proc. 25th Annual Conf. Eng. Med. Biol. 14, $159(1972)]$. The conductor elements, $0.3 \mu \mathrm{m}$ thick, are formed on both sides of a thin-film plastic substrate using vacuum deposition and photolithographic etching techniques. In the present configuration, the array consists of 33 platinum conductors, $12.7 \mu \mathrm{m}$ wide, each terminating at an electrode surface, $250 \mu \mathrm{m}$ wide. The electrodes are spaced on $700 \mu \mathrm{m}$ centers. Substrates investigated include Mylar, Parylene-C, and FEP and TFE Teflon films, 50 to $130 \mu \mathrm{m}$ thick. Of these, Mylar was found best for electrode fabrication. Insulation of the array is accomplished by dip coating in Silastic. The electrode tips are exposed by mechanical scribing techniques. Short-term body tolerance studies in guinea pigs show good compatibility with the electrode materials. Bipolar sinusoidal stimulation of the electrodes in normal saline shows a decrease in complex impedance proportional to the -0.99 power of the frequency: $23.8 \mathrm{M} \Omega$ at $100 \mathrm{~Hz}$ to $250 \mathrm{k} \Omega$ at $10 \mathrm{kHz}$. Stimulation studies in vitro and in vivo (scalae tympani of cats) are planned to determine behavioral responses to electrical stimuli and long-term tissue reaction and electrolytic dissolution effects. [This work was supported in part by Raytheon Company.]

S12. Placement of Electrodes for Activation of VIIIth Nerve. David Cowden aNd Richard Walloch, Department of Otorhinolaryngology, University of Oklahoma Health Sciences Center, Oklahoma City, Oklahoma 73190.-The goal of this study is to supplement recent attempts to use electrical stimulation as a hearing prosthesis in cases of sensorineural hearing loss by examining various placements for the stimulating electrodes. In the basal turn of an anesthetized guinea pig, two electrodes were placed in scala tympani and two were placed in scala vestibuli. A fifth electrode was placed in a neck muscle. These electrodes were used five times in each possible pairing to deliver bipolar pulses with a $0.5-\mathrm{msec}$ duration. The order of use was random. The threshold in microamperes for an evoked potential recorded from the contralateral auditory cortex and the threshold for a twitch in the facial muscles were obtained in a double blind manner. When two stimulating electrodes in the cochlea were employed, the threshold for an evoked potential was $156 \mu \mathrm{A}$ and $3610 \mu \mathrm{A}$ were needed before a muscle twitch could be detected. Different placements of the two electrodes within the cochlea did not significantly affect their performance. When a single intracochlear electrode and a muscle electrode were used for stimulation, $92 \mu \mathrm{A}$ were needed to evoke a cortical potential and $557 \mu \mathrm{A}$ caused a muscle of the face to contract. Because of the larger range, bipolar is preferable to monopolar stimulation of the auditory system. [Supported in part by the Deafness Research Foundation and the National Institute of Neurological Diseases and Stroke.]

S13. Changes in the Auditory Nerve Evoked Potential after Intense Sound Stimulation. CurT Mitchell, RoBerT Brummett, AND Jack Vernon, Department of Otolaryngology, Kresge Hearing Research Laboratory, Universily of Oregon Medical School, Portland, Oregon 97201.-Intense sounds were lound to produce a decrement in the nerve evoked potential (NEP) at a lower sound pressure level than that which produced a decrement in the ac cochlear potential. Both types of potentials were recorded from the round window of guinea pigs. Guinea pigs were exposed to pure tones of 3, 4, 6, and 12 $\mathrm{kHz}$, at 95-115 dB SPL (5 dB below the maximum cochlear potential output), for 15-30 min. These intensities produced no noticeable effect on the cochlear potential but produced a marked decrement (up to $30 \mathrm{~dB}$ ) in the NEP. The greatest loss in the NEP wa at $\frac{1}{1}$ oct above the stimulating frequency. These findings correspond to the reported shifts in hearing thresholds. 


\author{
Session T. Speech Communication IV : Speech Intelligibility and Perception
}

D.ANiel Beasley, Chairman

\author{
Michigan State University, Lansing, Michigan
}

\title{
Contributed Papers (7 minutes)
}

\begin{abstract}
T1. The Channel Capacity of the Ear as a Function of Frequency. E. L. R. Corliss, Institule for Basic Slandards, National Bureau of Standards, Washington, D. C. 20234.-The channel capacity of the ear as a function of frequency can be computed by a technique similar to that in an earlier paper [E. L. R. Corliss, J. Acoust. Soc. Amer. 50, 671-677 (1971)]. The legitimacy of this computation rests upon the justification that sufficient variables are involved to permit replacement of actual statistical distributions by normal distributions. 'This legitimacy is tested by comparing the predicted growth of intelligibility of individual phonemes as a function of level above threshold with experimental results. The channel capacity of the ear shows a broad maximum in the frequency range of $3-6 \mathrm{kHz}$. Taking into account the duration of speech sounds as a function of frequency, one finds that each phoneme tends to convey the same number of bits over the range from $300 \mathrm{~Hz}$ to $4 \mathrm{kHz}$. However, many more phonemes are uttered per unit time at the higher frequencies, so that the rate of information transfer is higher. This explains to some extent the empirical observation that the high-frequency limit necessary for accurate communication depends upon redundancy of the material. One ought to reconsider the convention that the spectrum above $3 \mathrm{kHz}$ is relatively irrelevant for speech communication when unfamiliar speech material must be recognized.
\end{abstract}

T2. Further Investigations of Reaction Time to Clicks in Sentences: Linguistic Structures Emerge with Extended Processing. K. H. Abrams, Program in Psycholinguistics, University of Michigan, Ann Arbor, Michigan 48104.-This study reports an experimental and theoretical resolution to two seemingly contradictory findings in the literature of attentional strategies of sentence processing. Abrams and Bever (1969) reported that reaction time (RT) to brief tones located at the linguistic boundary between major sentence constituents was slower than reaction time to tones near the major break within major constituents. Holmes and Forster (1970), using different sentences and different tone locations within constituents found that RT to tones between constituents was relatively fast. The present study used practiced subjects and a frequency balancing technique that equalized the conditional probability of a tone occurring in each of four locations given that a tone had not occurred previously in the sentence. In Condition I, the instructions emphasized fast reaction time and the results looked like the findings of Abrams and Bever. In Condition II, the instructions stressed written recall accuracy and the results replicated the Holmes and Forster findings.

T3. Discrimination of Spectrally Weighted Speech by HearingImpaired Subjects. InN B. Thomas and G. Barry PfanneBECKER, Electrical Engineering Deparlment, University of Massachusetts, Amherst, Massachusetls 01002.-Recent work by Martin, Osberger, and Pickett [J. Acoust. Soc. Amer. 52, 184 (A) (1972)] shows that for both normal and hearingimpaired subjects, $F 1$ causes considerable masking of $F 2$ transitions at the higher intensity levels typically necessary for the hearing impaired. Accordingly, we have processed normal speech to alter the relative intensities of $F 1$ and $F 2$ over a considerable range and presented this processed speech to hearing-impaired listeners. We have also presented normal (unweighted) speech to the same listeners at the same true rms level as a comparison. Spectral weighting was achieved by altering the attenuation slope of a high-pass filter having a cutoff frequency of $1600 \mathrm{~Hz}$. Slopes of $6,12,18$, and 24 $\mathrm{dB} /$ oct were considered. Intensities of weighted and unweighted speech were $20,30,40 \mathrm{~dB}$ above the speech reception threshold for unweighted speech. Results show that weighting usually increases discrimination for a given intensity level, but the most desirable attenuation slope changes as a function of intensity level. Discrimination gains are typically small $(<20 \%)$ and best in the marginal hearing conditions (20 and $30 \mathrm{~dB}$ SL). [Work supported by NIH.]

T4. Amplitude Compression. I. Speech Intelligibility in Quiet with Normal and Pathological Hearing Groups. S. VARgo and R. Carhart, Northwestern University, Evanston, Illinois 60201. - The theoretical contribution of amplitude compression to the enhancement of speech intelligibility for pathological hearing groups remains unresolved. Previous investigations by Caraway and Carhart [J. Acoust. Soc. Amer. 41, 1424 (1967)] and Burchfield et al. (1971) have shown contradictory findings on this topic. Although this study essentially replicates the two previous investigations, it employs a more advanced compressor system with an instantaneous attack time (100 nsec); moreover, word triplets are delivered to listeners at three sensation levels (SLs), 10, 20, and $30 \mathrm{~dB}$. The findings are discussed in terms of previous studies as well as in the usability of amplitude-compressed phonemic information at low SLs. [Supported by Special Research Fellowship Grant of NINDS.]

T5. Efficient Determination of Articulation Functions. D. L. BODE, Speech and Hearing Research Laboralory, Universily of Illinois, Champaign, Illinois 61820.-Previous experiments using adaptive methodology to measure monosyllabic articulation functions were described at the 80th and 81st Meetings of the Society [Bode and Carhart, J. Acoust. Soc. Amer. 49, 95(A) (1971); Bode, J. Acoust. Soc. Amer. 50, 146(A) (1971)]. These data indicated that (1) the use of a large test vocabulary (100 CNC words) results in highly stable and accurate measurement of a specific point on a function, and (2) adaptive tests theoretically can be used to calculate a standard error for an individual listener based on a single test administration. Subsequent experiments reveal that the use of a small test vocabulary ( 50 words) also allows highly reliable and valid point estimation. Administration of two tests aiming for $29.3 \%$ and $70.7 \%$ points identifies both the shape and location of the rising portion of articulation functions. Tests were administered to 24 normal-hearing subjects in quiet and in 60 and $80 \mathrm{~dB}$ SPL white noise. Practice effects were minimal, in most conditions averaging less than $1 \mathrm{~dB}$. Further, constant-level tests employed as validating criteria revealed that the adaptive measures were highly accurate (within a few percentage points) in identifying the preselected 


\section{TH MEETING ACOUSTICAL SOCIETY OF AMERICA}

measurement objectives on normal functions. [Research supported by National Institute of Neurological Diseases and Stroke.]

T6. Evaluating Communication Systems with Respect to Both Speech Intelligibility and Speaker Identification. C. E. Williams, Pensacola, Florida 32512, AND J. D. Mosko, U. S. Army Medical Research Laboratory, Ft. Knox, Kentucky 40121.-Communication systems which involve extensive speech processing may yield high intelligibility and yet fail to transmit characteristics associated with the talker (identity, vocal effort, expressive state, etc.). Some exploratory studies have been conducted to determine if the modified rhyme test (MRT) can be utilized to evaluate both the capability of communication systems to transmit intelligible speech and the features of talkers' voices that enable listeners to identify them. Tape recordings were made of each of five selected speakers reading different randomizations of MRT word lists. Single-speaker lists ( 50 words by a given speaker) and multiplespeaker lists (10 words by each of the five speakers, in random order) were constructed for presentation to listeners. Training tapes were also constructed to familiarize listeners with the speakers' voices. The training tapes and test tapes were processed through two vocoders and the output tapes, along with the unprocessed tapes, were presented to groups of listeners. Intelligibility scores obtained for the singlespeaker and multiple-speaker lists and speaker identification scores will be presented and discussed.

T7. Optimal and Preferred Listening Levels for Speech in Aircraft Acoustical Environments. R. M. RoBERTSON AND C. E. Williams, Naval A erospace Medical Research Laboratary, Pensacola, Florida 32512.--There is some question as to whether pilots and aircrew personnel routinely adjust the audio level of their radio receivers for maximum speech intelligibility and minimal damage risk to hearing. In an effort to answer this question, data have been obtained on the preferred listening levels (PPLs) of student naval flight officers in aircraft acoustical environments. Thirty subjects were given the task of tracking continuous speech in quiet and in the presence of three levels of background noise (70, 85 , and $100 \mathrm{~dB}$ SPL). They were asked to adjust the level of the speech for maximum intelligiblilty. Two different tracking procedures were employed: one utilizing a Békésy-type switch and the other an adjustable hold-type switch, similar to a normal radio gain control. PLLs obtained for the three noise conditions revealed that as the noise level decreased, the subjects preferred a more favorable signal-to-noise ratio. Normative PLL data and intelligibility test data for selected signal-to-noise ratios will be presented and discussed.

T8. Acoustic Alterations of Speech When Processed through Air Force Aircraft Communication Systems. D. C. Gasaway, $U$. S. Air Force School of Aerospace Medicine, Brooks Air Force Base, Texas 78235.- The acoustic composition of voiced speech signals received at a distance of $1 \mathrm{~m}$ (normal speech) is compared with those picked up by standard Air Force lip microphones that are transmitted via standard electroacoustic communication systems. The acoustic composition of speech used in Air Force ground and airborne operations is significantly different from that associated with normal live voice communication. Many voiced and voiceless speech components that constitute minimal acoustic amplitude in normal speaking situations undergo significant amplitude alterations when picked up by lip microphones. The author noted that pilots and other rated personnel frequently report that several transition flights are required before the speech communication messages are fully intelligible. This phenomenon has been studied and analyses of actual airborne speech communications are used to identify and define acoustic alterations that necessitate learning a new (or altered) type of speech. Voice prints of English vowels, diphthongs, and consonants are used to illustrate acoustic changes that exist between normal conversational speech and speech picked up by lip microphones. The results of this research suggest that the acoustic alterations created by picking up speech at the lips may increase intelligiblilty after the listener has learned how to process the signals accurately.

T9. Word Intelligibility of U. S. Navy Aircraft Radios. J. C. Wegster, Human Faclors Technology Division, Naval Electronics Laboralory Center, San Diego, California 92152.-Two types of in-flight recordings were made: (1) actual radio/ intercom voice messages on operational flights and (2) standardized word lists read by flying observers on routine test flights. An experienced civilian listener who lacked any experience or knowledge of air operations transcribed $34 \%$ of the operational recording, an experienced U. S. Navy Chief Petty Officer Air Controller $68 \%$, and an experienced U. S. Navy aviator $90 \%$. The standardized word lists were listened to by typical U. S. Navy enlisted men. The lists included the 1000 phonetically balanced (PB) words, the Fairbanks rhyme test (FRT), the modified rhyme test (MRT), the Voiers diagnostic rhyme test (DRT), Clarke's medial vowel rhyme test (VRT), and a specially developed brevity codephonetic alphabet word test (BREV). Scores on the FRT, $M R T$, and BREV words were equivalent and therefore the easier-to-administer standardized tests (FRT or MRT) can be considered valid alternatives to words actually used operationally. Scores on the PB tests were low, and scores on the VRT and DRT were high. Analysis of the diagnostic words showed the most frequent consonant pair confusion on aircraft radios to be $\mathrm{V} / \mathrm{B}$; the vowel $\mathrm{EE}$ (beat) was the worst "carrier" vowel, while EH (bet) was the best. Nasality was an intelligible attribute at good speech-to-noise $(\mathrm{S} / \mathrm{N})$ ratios but very bad under bad listening conditions. Voicing and sibilation were the more intelligible attributes when listening at low $\mathrm{S} / \mathrm{N}$ ratios.

T10. Intelligibility of Initial versus Final Consonants in Monosyllabic Words. V. W. ByErs, Audiology Clinic, LSU Medical Center, New Orleans, Louisiana 70119.-The intelligibility of 10 consonants $[/ \mathrm{p} /, / \mathrm{t} /, / \mathrm{k} /, / \mathrm{b} /, / \mathrm{d} /, / \mathrm{g} /, / \mathrm{m} /$, $/ \mathrm{n} /, / \mathrm{l} /$, and $/ \mathrm{s} /]$ in two-vowel environments, $/ \mathrm{l} /$ and $/ \mathrm{ae} /$, were studied in the initial and final positions of monosyllabic words. A closed set of 88 words was constructed such that the consonants were transposed within the same vowel environment, e.g., pat-tap. Three randomized lists were presented at $60 \mathrm{~dB} \mathrm{HL}$ to subjects in a counterbalanced order of three signal-to-noise ratios, $+4 \mathrm{~dB}, 0 \mathrm{~dB}$, and $-4 \mathrm{~dB}$. Results indicated that the initial consonant is more intelligible than the final consonant. Additionally, significant differences were found among the subjects, vowel environment, and individual phonemes. The identification of a monosyllabic word is a complex process in which the distinctive features that serve as perceptual cues for the initial and final consonant may not be processed in a similar manner.

T11. Intelligibility of Alternated Speech. T. T. TroOIEN AND C. SpeAks, Department of Communication Disorders, University of Minnesota, Minneapolis, Minnesota 55455.-The intelligibility of interaurally alternated speech was measured by two methods: shadowing and subjective rating. The first of two experiments used speech spoken at a normal rate; the second experiment used fast-rate speech. With normalrate speech, shadowing scores and estimates of intelligibility by eight subjects were nearly $100 \%$ for eight different rates of alternation between 1 and 10 aps. Estimates of intelligibility for fast-rate speech were depressed slightly from $100 \%$ and shadowing scores were near $70 \%$ for most rates of alternation. 
No particular alternation rate or region of alternation rates produced significantly depressed shadowing scores. The results convince us that interaural alternation of speech does not affect intelligibility unless the speech is spoken at a very rapid rate. Even with fast-rate speech, however, intelligibility is not dependent in any meaningful sense on the rate of alternation.

T12. Interference Test Evaluations of Telephone Speech, Quality. L. H. Nakatani AND K. D. Dukes, Bell Laboratories, Murray Hill, New Jersey 07974.-The interference test for speech quality evaluation was investigated for sensitivity and validity with various types of telephone speech. Three components of the telephone system (microphone, transmission channel, and earphone), each with two alternatives (high fidelity or telephone), were used in all possible combinations to obtain eight types of telephone speech. The interference test was sensitive enough to measure the amount by which each telephone component degraded speech, even though all the speech types were highly intelligible according to articulation tests. Consistent and systematic relationships were observed between the interference test results, articulation test scores, and subjective evaluations of the same speech types, indicating that the interference test is a valid procedure for measuring speech quality. Moreover, by simply adding up the interference test measures associated with the telephone components, the measure for a speech type with any combination of the components could be accurately predicted. The results confirm that the interference test yields sensitive, valid, and additive measures of speech quality.

T13. Speech Reconstituted from Spectra of Reduced Dimensionality: A Study of Intelligibility. K.-P. L1 AND G. W. Hughes, Purdue University, West Lafayelle, Indiana 47907, AND A. S. House, Institute for Defense Analyses, Princeton, New Jersey 08450 .- In this preliminary study, we attempt to relate the space defined by a statistical [principal component] analysis of speech spectra to perceptual space represented by intelligibility scores. Speech spectra were represented in terms of a varying number of dimensions $[3,5,7,10,15$, and 35], each corresponding to a different degree of statistical accuracy, and the waveform was reconstituted from the reduced spectral data by means of inverse FFT computation [K.-P. Li, G. W. Hughes, and A. S. House, J. Acoust. Soc. Amer. 49, 134(A) (1971)]. Consonantal intelligibility scores for isolated monosyllabic utterances by one talker were determined from the responses of 12 listeners to a total of 553 test items. The average score increases monotonically from $47 \%$ to $75 \%$ as the spectral dimensionality varies from 3 to 35 . Along with the number of dimensions, manner and place of consonant articulation and the effects of the details of the reconstitution process enter into the relationship between statistical accuracy and intelligibility.

T14. Relative Effects of White and Speech-Correlated Noise on Speech Quality. T. K. SEn and T. M. DenNis, Bell Laboralories, Holmdel, Nezw Jersey 07733.-An experiment was designed to study the effects of speech and noise levels on speech quality using white and speech-correlated noise (SCN). Two types of speech signals were used: (1) flat with respect to frequency (flat speech), and (2) flat below $1 \mathrm{kHz}$, and with frequency emphasis of $9 \mathrm{~dB}$ /oct above $1 \mathrm{kHz}$ (shaped speech). Both speech and noise were bandlimited between 150 and $3600 \mathrm{~Hz}$. The speech level ranged between 54 and $100 \mathrm{dBt}$ SPL and the noise spectrum level between 2 and $33 \mathrm{dBt} /$ Hz. A total of 45 noise and speech level combinations were used as test stimuli. Each test condition consisted of a pair of phonetically balanced sentences in the presence of one of the 45 test stimuli. The subjects were required to rate the speech quality on a 5 -point scale. Averaged over all conditions, the shaped speech was preferred over flat speech. For shaped speech, SCN was preferred over white noise. For flat speech the same was true for speech levels of about $80 \mathrm{dBt}$ and higher, and the reverse was true for speech levels lower than $80 \mathrm{dBt}$,

WEDNESDAY, 29 NOVEMBer 1972

EL DoRado West, 2:00 P.M.

\title{
Session U. Physical Acoustics III : Ultrasonic Energy and Power Measurements II
}

\author{
Martin Greenspan, Chairman
}

National Bureau of Standards, Washington, D. C. 20234

\section{Invited Papers}

2:00

\section{Radiation Force Method Tutorial Paper}

U1. Acoustic Radiation Pressure and its Use in Power and Intensity Determinations. J. A. RooNey, Physics Department, University of Maine, Orono, Maine. - The radiation force upon an object placed in a sound field can be used to determine the acoustic power or intensity. Two types of test objects have been extensively used as targets in radiation pressure determinations. The first is either an absorbing or reflecting target which intercepts the entire sound beam. For the case of a traveling plane wave in an open vessel impinging on an absorbing target, the radiation force $F$ equals the acoustic power divided by the velocity of sound $c$ even when nonlinearities of the propagation medium are taken into account. The second type of test object used for calibration is a sphere. For an elastic sphere of radius $a$ surrounded by a nonviscous fluid in a traveling plane wave $F=Y \pi a^{2} I / c$. Here $I$ is the local intensity and $Y$ is the acoustic radiation force function. Experimentally the forces on targets have been determined by observations of deflections for pendulum arrangements, measurement of change in target weight using analytical balances, measurement of change in displacement of targets attached to floats, and determination of the force necessary to maintain the target in equilibrium. For measurements of low acoustic powers, it is necessary to minimize fluctuations associated with building vibration, convection currents, and interfacial effects. [Work supported by HAS project at the University of Vermont and by the National Institutes of Health.] 
Radiation Force Method Panel Discussion

F. DuNN Bioacoustics Research Laboratory, University of Illinois, Urbana, Illinois 61801

F. J. FRY Indiana University Schaol of Medicine and Interscience Research, Institute of the Indianapolis Center for Advanced Research, Indianapolis, Indiana 46202

J. E. PIERcy Physics Division, National Research Council, Ottawa, Ontario, Canada

J. M. ReID Providence Hospilal, Seaule, Washington 98122

\title{
Optical Method Tutorial Paper
}

U2. Ultrasonic Energy Measurement by Optical Diffraction Technique. BILL D. CоOK, Cullen College of Engineering, University of Houston, Houston, Texas 77004.-Optical methods are often proposed for the precise determination of the energy density of an ultrasonic area. Several measurement techniques have been developed to yield certain optical parameters. However, the difficulty remains in interpreting the results in terms of the local parameters of the sound field. This paper reviews the basic concepts of ultrasonic light diffraction, the parameters used to describe these concepts, methods of measurement, and limitations on interpretation.

\section{4:00}

Optical Method Panel Discussion

L. E. Hargrove Crawford Hill Laboratory, Bell Laboratories, Holmdel, New Jersey 07733

A. KoRPEL Research Department, Zenith Radio Corporation, Chicago, Illniois 60639

W. G. MAyer Physics Department, Georgetown Universily, Washington, D. C. 20007

WEDNESDAY, 29 November 1972

EMPIRE ROOM, $2: 00$ P.M.

\section{Session V. STOL Noise}

\author{
R. C. Chanaud, Chairman \\ Department of Civil and Environmental Engineering, \\ University of Colorado, Boulder, Colorado 80302
}

\section{Invited Papers (30 minutes)}

\begin{abstract}
V1. Noise Considerations for STOL Vehicles. David ChestnutT, NASA, Langley Research Center,
\end{abstract} Hampton, Virginia 23365.-[No abstract available.]

\begin{abstract}
V2. Fundamentals Regarding Reduction of Noise Arising from Jet Interaction with Deployed LiftAugmenting Flaps. Richard E. HAYDEN, Boll Beranek and Newman Inc., 50 Moulton Streel, Cambridge, Massachusetts 02138. - Proposed lift-augmented STOL aircraft are expected to require noise reductions on the order of $20 \mathrm{~dB}$ to meet community noise goals, according to a number of recent model studies. A predominant source of noise associated with the concepts and configurations currently under consideration is the interaction of the exhaust stream with deployed flaps. This flow-surface interaction produces both large-scale aerodynamic force fluctuations, which radiate like point dipoles at low frequencies, and smaller-scale surface pressure fluctuations, which radiate only from the leading and trailing edges of these surfaces. The fundamentals of the relevant noise-producing processes are reviewed and basic noise reduction concepts developed using parametric source models. Experimentally observed noise reductions on a 1/15 scale externally blown flap (EBF) system are presented for two of the noise reduction concepts-modification of the acoustic transduction process using variable impedance surfaces, and modification of the incident flow field through introduction of bigh porosity mesh grids upstream of the flaps.
\end{abstract}

\section{Contributed Papers (12 minutes)}

V3. Acoustic Results Obtained with an Upper-Surface-Blowing Lift-Augmentation System. U. von GLAHN, M. REshotKo, AND R. Dorsch, Jet Acoustics Branch, NASA Lewis Research Center, Cleveland, Ohio 44135. - The noise caused by the interaction of the exhaust jet and the wing was measured under static conditions for several versions of a small-scale
STOL engine-over-the-wing configuration. Three basic nozzles were tested: a circular nozzle with deflector, a $5: 1$ slot nozzle with and without deflector, and a 10:1 slot nozzle. The wing included a flap lift-augmentation system with flap deflection angles of $20^{\circ}$ for a takeoff condition and $60^{\circ}$ for an approach condition. Far-field noise data are presented for nominal jet 
pressure ratios of 1.25 to 1.7 for the flyover mode. The data are discussed in terms of SPLs and normalized sound pressure spectra: comparisons with nozzle-only data are also made. The model OASPL near $90^{\circ}$-measured from an engine inlet - follow a 6 th-7th power relationship with jet velocity compared to an 8th-9th power law for the jet alone. Only minor acoustic differences were obtained between the two flap deflection positions. The most significant noise reduction was achieved with the 10:1 slot nozzle. Implications of extending the small-scale model acoustic data to a full-scale aircraft are discussed and indicate a sizable noise attenuation may be achieved owing to wing shielding.

V4. Wind-Tunnel Studies of Helicopter Rotational Noise in Forward Flight. Shelia Widnall, Department of Aeronautics, MIT, Cambridge, Massachusetts 02138.- Some results of wind-tunnel studies of helicopter noise carried out on a model rotor operating in a large open-jet wind tunnel are described. The tunnel test section is surrounded by an anechoic chamber. Data processing techniques were used to obtain the averagerepeated-transient signal per revolution and thus to extract the rotational noise from the total noise, which includes random components. Blade-vortex interaction is clearly visible in many of the repeated-transient signals and is felt to be an important contributor to rotational noise even outside of the blade-slap condition. Vortex noise can be identified in the spectra of the total signal. In addition to the vortex noise, a broad-band noise of lower frequency is evident. It is postulated that this lower-frequency noise is due either to vortexwave unsteadiness or to local stall on the blades at certain azimuth locations.

V5. Reduction of Noise Generated by Flow of Fluid over Plate. B. Pinkel and T. D. Scharton, Bolt Beranek and Newman Inc., Canoga Park, California 91303.-This paper describes a method of reducing the noise generated by a fluid stream flowing subsonically over the edge of a plate and also presents some substantiating test data. This concept may prove useful for reducing the noise of STOL aircraft employing the externally blown flap or the augmenter wing, which configurations are now under exploratory development.

V6. Near-Field Environments of VTOL Jets. L. C. SUThERLAND, Wyle Laboralories, 128 Maryland Sireet, El Segundo, California 90245.-For jet-powered VTOL aircraft, the principal sources of nearfield noise are the direct and groundreflected noise of the jet exhaust, the aerodynamic noise from wall jet formed by the deflected exhaust flow and the noise of the impinging jet. These principal sources are considered and several of their basic characteristics for VTOL jet noise are established. Emphasis is placed on the change in acoustic power and near-field levels due to their combined effect. A prediction method for near-field noise of VTOL jets is then outlined in the form of additive corrections to apply to nearfield levels for the undeflected jet. These corrections have a maximum value of about $15-20 \mathrm{~dB}$ in the range of typical resonance frequencies for flight vehicle skin panels. However, these additive corrections vary substantially with frequency and elevation of the vertically directed nozzle. The approximate trend in these variations is illustrated based on the results of model studies. [This work was supported by the Air Force Flight Dynamics Laboratory, Wright--Patterson Air Force Base, Ohio.]

V7. A Comparative Study of Augmentor Wing, Ejector Nozzle, and Power Jet Flap Low-Noise STOL Concepts. J. V. O'Keefe, T. B. Nickson, and H. C. True, New Products Technology, Conmercial Airplane Group, The Boeing Company, Seattle, H'ashington.-A noise and performance comparison of these STOL airplane configurations is summarized. Aircraft were sized for a 2000 -ft takeoff field length, 150 passengers, and a STOL range of 500 miles. A discussion of thrust augmentation ratios, airplane "thumb-print" sizing, thrust requirements, thrust lapse rates, static test configurations, $500-\mathrm{ft}$ SL noise comparisons, and noise "footprints" is presented for each concept. Each concept originally showed approximately equal noise levels on a static test stand but more inteusive test work shows that the augmentor holds superior potential for low noise.

V8. Far Noise Field of a Subsonic Air Jet. J. C. Yu, Civil, Mechanical and Environmental Engineering Department, The George Washington University, Washington, D. C. 20006.-Far noise field survey was made for a 2 -in. circular subsonic cold air jet. The exit velocity range investigated varied from 350 to $800 \mathrm{ft} / \mathrm{sec}$. The experiment was conducted in the open field. The contoured converging nozzle used had a contraction ratio of 50. In order to eliminate possible upstream acoustic contamination of the jet noise, blown-down technique was used to generate jet flow with a large acoustically treated settling chamber. Experimental results obtained are presented in the form of overall and spectral directivity, power spectrum, total power radiated, and their dependence on mean flow parameters. The experimental findings were correlated with the existing theories of subsonic jet noise. [This study has been sponsored, by the NASA-Langley Research Center.]

\title{
Session W. Underwater Acoustics III : Environmental Relationships in Ocean Sound Propagation
}

\author{
Alan O. Sykes, Chairman
}

304 Mashie Drive, S.E., Vienna, Virginia 22180

\section{Invited Papers}

W1. Long-Term Sound Propagation Studies in the Bristol Channel. D. E. Weston, Admiralty Research Laboratory, Teddington, Middlesex, England.- Sound propagation has been studied for several years in the shallow waters of the Bristol Channel. The measurements central to this account have involved repeated transmissions of a sequence of tone pulses, with frequency varying from 0.3 to $4.5 \mathrm{kHz}$, the arrivals building up into a simple display. The signals were transmitted and monitored by fixed bottom-laid transducers, with path lengths up to $137 \mathrm{~km}$. The investigations have been sup- 
ported by other time-series measurements : in long-range echo ranging on fish and other reverberators, in ambient noise, and in oceanography. As a result of prolonged detective work, 11 different mechanisms causing signal fluctuation have been identified, eight of which would not have been revealed in the absence of long time-series results. Four reasons for the importance of long-term fixed-transducer multiple-frequency studies are given, as follows, each illustrated by one of the fluctuation types : (a) Many effects need relatively long studies if they are to be seen at all, e.g., the diurnally changing attenuation due to fish. (b) Some effects may only be understood by their behavior over long periods, e.g., fuctuations due to internal waves and their tidal dependence. (c) Some effects occur at random times, to be observed only by patient waiting, e.g., storms. (d) Understanding of the absolute transmission levels involves a knowledge of the variations in level, especially the variations with season.

W2. Fluctuations of Sound Transmission in the Straits of Florida. John C. STEINBErg, Institute for Acoustical Research, Miami Division of Palisades Geophysical Institute, Miami, Florida 33130.Sound waves transmitted across the Florida Current between fixed transducers are used to investigate relationships between acoustic and environmental variations. Year-long measurements of the amplitude and phase of $420-\mathrm{Hz}_{2}$ sound waves reveal fluctuations with periods from seconds to weeks and longer-term trends. The variations of phase correspond to changes in the medium and increase in magnitude with increase in period. The amplitude fluctuations are not readily identifiable. The slowly changing phases of the multipath arrivals result in varying patterns of amplitude interference and fading which have noiselike spectra. The Florida Current is characterized by a cross-stream temperature-depth structure related to the transport of water. Variations of transport from tidal and wind forces induce fluctuations of temperature, sound speed, and phase. Temperature and phase are also modulated by internal waves and components of current parallel to the sound path. The observed characteristics of the amplitude and phase variations appear in computations with ray-propagation models in which plane parallel boundaries and measured time variations of sound speed versus depth are used.

W3. Sound Transmission in Moving Media with Application to the BIFI Range. L. A. Stallworth, New London Laboratory, Naval Underwater Systems Center, New London, Connecticut 06320, AND M. J. Jaconson, Rensselaer Polytecknic Institute, Troy, New York 12181.- The effect of currents on sound transmission is examined for three ocean channel models: constant sound speed and spatially uniform current, linear depth-dependent sound speed and spatially uniform current, and constant sound speed and current of constant direction and linear depth-dependent magnitude. Ray theory is used to analyze the effect of current on ray path geometry, spreading loss, boundary loss and phase shift, and travel time of individual arrivals at a fixed receiver. The arrivals are summed to determine the effect of current on the acoustic field for each model. The results are applied to the BIFI range, a 33-km $\times 40-\mathrm{m}$ channel. The theoretical calculations are compared with continuous observations of the received phase of an acoustic signal over a 48-h period when the first model is applicable and with intermittent measurements of propagation loss when the second model is applicable. In the third model, a tidal current is shown to produce the phase offset-amplitude fade phenomenon, as well as other acoustic fluctuations having a much shorter period than the tidal current.

W4. Surface-Duct Propagation-Loss Measurements. James Cummins, Naval Undersea Center, San Diego, Califormia 92132. - The surface duct is the mixed-layer underwater sound channel generally bounded by the ocean surface and the thermocline. In a recent experiment, abundant measurements of surface-duct propagation loss were made concurrently with detailed environmental sampling. The experiment requiring three ships was designed to fill a gap in the knowledge of surface-duct propagation at frequencies below $8 \mathrm{kHz}$ that has not been addressed. Propagation loss was measured at frequencies from 0.4 to $20.0 \mathrm{kHz}$, at depths from 20 to $600 \mathrm{ft}$, over ranges of $0-18$ miles. The experiment was novel because for the first time adequate environmental measurements were made over the entire acoustic field. Three different instruments measured sea surface roughness. Sound speed profiles were continuously sampled both in space and time: every $50 \mathrm{ft}$ along the track by a towed thermistor chain, every $10 \mathrm{sec}$ by a string of thermistor buoys spaced along the propagation path. The accuracy of these systems was $\pm 0.02^{\circ} \mathrm{C}$ or $\pm 0.06 \mathrm{~m} / \mathrm{sec}$ in terms of sound speed. The purpose of the experiment was to provide data required for evaluating several theories of surface duct propagation loss.

W5. Sound Propagation in a Surface Duct with a Rough Surface. H. P. Bucker, Naval Undersea Center, San Diego, California 92132, AND J. L. Thompson, Royal A ustralian Navy Research Labaratory, Sydney, A ustralia.-Sound propagating in a surface duct is partially scattered by surface reflections. This can be accounted for in propagation models by first using a surface reflection coefficient with modulus less than one and then by adding a ray theory correction representing sound scattered directly from the surface to the receiver. An outline of the theory is presented, and calculated propagation loss curves are compared to experimental values taken during recent sea observations.

\section{Contributed Papers (10 minutes)}

W6. Amplitude Fluctuations in Shallow Water Acoustics. P. A. Barakos, Naval Undersea Research Development Center, San Diego, California 92132.--Large amplitude and phase fluctuations in underwater acoustic wave fields occur and are primarily due to the temporal and spatial variability of the water and its boundaries. The amplitude part of this phenomenon is investigated by obtaining, simultaneously, time and space series of environmental and acoustic data from sensor arrays fixed in space, in shallow coastal water off southern Califormia. The signal output from a nuclear powered ultra- 
sonic source, mounted on a tripod $3 \mathrm{~m}$ above the bottom is monitored continuously by a hydrophone $150 \mathrm{~m}$ away. The source radiates three $\mathrm{CW}$ pulses every $3 \mathrm{sec}$, sequentially at 30,45 , and $60 \mathrm{kHz}$. A sampling interval of $3 \mathrm{sec}$ was used. Time series of temperature from a vertical thermistor array, surface waveheight, and the two horizontal components of water motion from an EM current meter are obtained. Two dominant frequencies, one associated with the surface swell and the other with the internal waves in this area, are found in both the acoustic and the environmental data. Statistical and deterministic techniques are employed to clarify the interaction between acoustic and environmental wave fields.

W7. Ray Propagation in Slowly Time Varying Deep Ocean Channels. I. N. L. Weingerg, R. P. Flanagan, and J. G. Clark, Institute for Acoustical Research, Miami Division of Palisades Geophysical Institute, Miami, Florida 33130.-Ray acoustics in deep ocean channels is being studied with the specific objective of describing the influence of low-frequency internal waves and surface tides upon multipath propagation. The work is a joint study in which the ray model is coordinated with a fluid dynamical investigation of the effect of internal tides upon deep ocean sound speed stratification. The geometry of the propagation channel consists of parallel boundaries and a composite sound speed profile with straight line segments in the main thermocline and deep water regions, and two hyperbolic cosines in the vicinity of the SOFAR axis with the slopes continuous at all junctions. For arbitrary source and receiver locations, the initial angles of all the ray arrivals were determined. Using closed form expressions for each segment, travel time and spreading loss were calculated from which amplitude and phase can be found. Complete descriptions of the propagation multipaths are given for selected examples which combine the several types of rays encountered in deep ocean transmission. In a subsequent presentation (Part II), the time variations in these multipaths due to tidal fluctuations in the sound structure are discussed.

W8. Sound Speed Perturbations Due to Internal Tides, Christopher N. K. Mooers, Division of Physical Oceanography, Rosenstiel School of Marine and Almosphere Science, Universily of Miami, 10 Rickenbacker Causeway, Miami, Florida 33149.- Internal tides are perhaps the most prevalent, and largest amplitude, internal gravity waves in the ocean. They are commonly generated by the scattering of surface tidal energy into internal modes when the surface tides propagate on to continental shelves. A portion of the internal tidal energy propagates into the deep ocean. One of the effects of internal tides on underwater acoustic propagation is to perturb the temperature and salinity versus depth profiles, and, thus, the sound speed versus depth profile. Starting with temperature and salinity versus depth profiles from near Eleuthera Island, the internal tide eigenfunctions, as well as the sound speed profile, have been computed for a water depth of $4500 \mathrm{~m}$. Assuming a value for the vertical displacement amplitude of a first vertical mode internal tide, the perturbed temperature and salinity profiles have been computed. From these perturbed profiles, the consequent perturbed sound speed profiles have been computed for cases of maximum up and down internal tide displacement. The first mode internal tide has no vertical displacement at the sea surface and bottom and a maximum near the base of the main thermocline (depth of about $1200 \mathrm{~m}$ ). Since the SOFAR axis is also near the base of the main thermocline, and since the pressure effects on sound speed are predominant below the SOFAR axis, the sound speed perturbation due to the first mode internal tide is greatest at a depth (about $550 \mathrm{~m}$ ) above the SOFAR axis. An internal tide with a maximum vertical displacement of $10 \mathrm{~m}$ produces a maximum sound speed perturbation of $\pm 0.7 \mathrm{~m} / \mathrm{sec}$.

W9. Ray Propagation in Slowly Time-Varying Deep Ocean Channels. II. J. G. Clakk and N. L. Weinberg, Institute for Acoustical Research, Miami Division of Palisades Geophysical Institute, $615 \mathrm{~S}$. W. 2nd Avenue, Miami, Florida 33130. - Results from the studies described in the two preceding abstracts have been combined in models that describe the effects of internal tides and surface tides upon multipath acoustic propagation. Deep ocean experimental data that motivated this analysis will be discussed briefly. Several examples of ray multipath propagation in deep ocean channels time-varying under the influence of internal and surface tides will be examined. Comparisons will be drawn with analogous results from shallow water studies.

W10. Scattering Function Measurements for a 7-NM Propagation Range in the Florida Straits. H. A. DEFErRarl AND Lan Nghiem-Phu, University of Miami, Rosenstiel School of Marine and Atmospheric Science, 10 Rickenbacker Causeway, Miami, Florida 33149. - A pulse received after transmission through the ocean will be spread in time owing to multipath and shifted in frequency owing to scatter from the ocean wave surface. If the time spread is small compared with the period of the surface waves, a Doppler spectra may be measured for each successive part of the received signal. The resulting scattering function is a three-dimensional description of the transmission (intensity, frequency, and delayed time of arrival). Scattering functions for the 7-NM range show that energy arriving via SRBR paths has Doppler spectra with sideband components at surface wave frequencies. A large fraction of the total received energy is transmitted by RBR paths and is not influenced by surface waves. Measurements are presented for some environmental conditions typical of the Florida Straits. Results are compared with predictions from combined propagation and scattering models that include the effects of multiple scattering along the propagation paths.

SOCIAL HOUR 6:30 P.M.

Banguet 7:30 P.m. (Carillon Room)

Presentation of the

Distinguished Service Citation to

LAURENCE BATCHELDER 


\title{
Session X. Psychological Acoustics III : Binaural Interactions and Localization
}

\author{
Constantine Trahiotis, Chairman
}

\author{
Psychology Department, University of Illinois, Urbana, Illinois 61801
}

Contributed Papers (10 minutes)

\begin{abstract}
X1. Loudness Summation between Tones from Two Loudspeakers. B. SchARF, Northeastern Universily, Boston, Massachusetts 02115.-Loudness summation between two equally loud, $500-$ msec tones centered on $2000 \mathrm{~Hz}$ was measured in an anechoic room as a function of the frequency separation, $\Delta F$, and onset time difference, $\Delta T$, between the tones. One tone was presented via a loudspeaker $3 \mathrm{~m}$ from the subject and $45^{\circ}$ to the left of his median plane, the other tone via a loudspeaker $45^{\circ}$ to the right. A comparison $2000-\mathrm{Hz}$ tone was presented via a third speaker in the median plane. Overall loudness increased with $\Delta F$ once the critical band was exceeded. When $\Delta T$ was $0 \mathrm{msec}$, loudness summation at 45 , 65 , and 85 phons was very similar to that between two tones presented through a single earphone. As $\Delta T$ increased from 0 to about $100 \mathrm{msec}$, loudness summation declined gradually; beyond $100 \mathrm{msec}$, it declined more rapidly. The Haas or precedence effect was evident at $\Delta T$ 's less than 40 to 60 msec and $\Delta F^{\prime}$ 's less than about $1000 \mathrm{~Hz}$, where the six subjects usually heard a single sound originating toward the side of the leading loudspeaker.
\end{abstract}

X2. Binaural Interaction with Stimuli that Produce Periodicity Pitch. E. R. Hafter and G. L. Ricard, Department of Psychology, University of California, Berkeley, California 94720.-Periodicity pitch was produced by $100 \%$ modulation of bandlimited white noise. The periodicity waveform was rectangular with a $500-\mathrm{Hz}$ repetition rate and a $10 \%$ duty cycle. Two gates were used to present the modulated noises separately to the two ears. The gates carried the same noise, but the modulation waveforms were delayed by $\Delta t \mu \mathrm{sec}$ so that the noise came on first in the leading ear and went off last in the lagging ear. Perception of these stimuli and the consequent detection of $\Delta \ell$ was shown to be dependent on the frequencies of the carrier: for low-frequency noise, the lateralized percept was on the side of the leading ear, while for the high-frequency noise it was on the side of the lagging ear. A model that describes these results interprets the lateralization obtained with the low-frequency carrier as responses to the onset of the dichotic bursts and the lateralizations obtained with the high frequency carrier as responses to the offsets of the bursts.

X3. Binaural Detection Models: Varying Signal Parameters, Noise Identical at the Two Ears. E. Osman, Department of Psychology, Brooklyn College, Brooklyn, New York 11210.-A previously published statistical decision model of binaural detection called the correlation model [J. Acoust. Soc. Amer. 50, 1494-1511 (1971)] is evaluated further for data obtained with tonal signals in noise, where noise is identical at the two ears and of constant amplitude while signal parameters are varied. Equations are based on the model's expressions for the conditional density functions. Predictions of BMLDs (binaural masking level differences) for variation of interaural amplitude ratio and phase of signal are evaluated with regard to data of Egan [J. Acoust. Soc. Amer, 38, 1043-1049 (1965)] and Colburn and Durlach [J. Acoust. Soc. Amer. 38, 93-103 (1965)]. Comparisons of the correlation model, the equalization-cancellation model [Durlach, J. Acoust. Soc. Amer. 35, 1206-1213 (1963)], and a lateralization model [IJafter, J.
Acoust. Soc. Amer. 50, 1116-1122 (1971)] are discussed. Predictions for sets of psychometric functions resulting from binaural detection under several interaural listening conditions are compared to data and psychometric functions presented in Egan [Perception Psychophys. 6, 209-215 (1969)]. [Supported by NIH and CUNY.]

X4. Binaural Interaction between Real Tones and the Cubic Distortion Product, $2 f_{1}-f_{2}$. E. R. HAfTer, Deparlment of Psychology, University of California, Berkeley, 94720, AND B. S. Leshowirz and G. S. Jevinins, Department of Psychology, Arizona State Universily, Tempe, Arizona 85281.- Several investigators have shown that the amplitude of the cubic difference tone, $2 f_{1}-f_{2}$, does not follow the rules of nonlinear distortion observed for stimuli at high levels. They have concluded that the site of the distortion is certainly higher than the middle ear and perhaps central to the mechanical filtering of the basilar membrane. In this work, we further examine the place of generation by asking whether the cubic difference tone is subject to binaural interaction. To this end, two primary tones at frequencies $f_{1}$ and $f_{2}$ were presented to one ear while a third tone at frequency $f_{2}\left(f_{3}=2 f_{1}-f_{2}\right)$ was presented to the other ear. In a two-alternative forced choice, subjects were able to discriminate differences in the phase of $f_{\mathrm{a}}$. This demonstration shows that the distortion tone is produced distal to the neural mechanisms of binaural interaction. One advantage of our technique is that it does not confound the distortion stimuli with the addition of probe tones. Consequently, binaural interaction provides a potentially sensitive technique for measuring the level and phase of distortion products.

X5. Detection of Time Differences and Level Differences from a Position away from the Median Plane. Llovd A. Jeffress and Alan D. Sharpley, Department of Psychology and Applied Research Laboratories, The University of Texas at Austin, Austin, Texas 78712.--By the use of an interaural phase difference of $90^{\circ}$, a $50-\mathrm{Hz}$ band of noise, centered at $500 \mathrm{H}_{2}$ was delayed to the right ear by $0.5 \mathrm{msec}$. The resultant sound was perceived as lying well to the left of the median plane. To this continuous noise was added a signal from the same noise source so arranged in phase that the signal plus masker appeared to lie either inward or outward from the continuous masker. The signal plus masker could also be moved inward or outward by introducing a difference of level into the two earphone channels. Detection of these movements was compared with detection of movements away from the median plane by employing the $N_{0} S_{*}$ condition with $\alpha=90^{\circ}$ to obtain a time difference, or $\alpha=0^{\circ}$ to obtain a level difference relative to the median plane. The results show that detection of a time difference from a position away from the median plane is inferior to detection of a time difference around the median plane. However, the results for a difference of level showed no such advantage for the median plane; detection was the same from both reference positions. The findings are taken as further indication of the relative independence of the time mechanism and the level mechanism of the auditory nervous system. [Supported in part by a 
grant from the Public Health Service, National Institute of Neurological Diseases and Stroke.]

X6. Detection of Interaural Time Differences in ShortDuration Low-Frequency Tones. G. L. RICARD AND E. R. HAFTER, Department of Psychology, Universily of California, Berkeley, California 94720.--The detectability of interaural time differences $(\Delta l)$ was investigated as a function of stimulus duration, level, and frequency. Temporally dichotic tone bursts of 250,500 , and $1000 \mathrm{~Hz}$ were presented in a two-interval forced choice, in such a way that the signal to one ear led by $\Delta t \mu$ sec during either the first or second interval. The tone bursts were $10,25,50,100$, and 205 msec in duration and were gated with a rise-decay time of $2.5 \mathrm{msec}$. The levels used were 50 and $70 \mathrm{~dB}$ SPL. Threshold values of $\Delta t$ were maximal for the shortest durations, then fell rapidly to asymptote in the region from 50 to 100 msec. In addition, thresholds decreased at higher frequencies. Finally, all conditions showed lower thresholds for $70 \mathrm{~dB}$ than for $50 \mathrm{~dB}$. The data are fit by a model that treats increased duration as an increase in the number of samples of the interaural difference and describes the improvements found with higher frequencies and levels as a result of the steeper slopes of the displacement waveforms.

X7. Binaural Processing in the Cat. I. Lateralization. O. S. WAKEFORD, Instilute for Basic Standards, National Bureau of Standards, Washington, D. C. 20234, Aivd D. E. RoBinson, Department of Psychology, Indiana University, Bloomington, Indiana 47401.- The ability of the cat to "lateralize" tonal signals having interaural intensive or interaural temporal disparities was measured. Interaural intensive differences were studied at $0.5,1.0$, and $3.0 \mathrm{kHz}$. Interaural temporal differences were studied at $0.5,1.0,2.0$, and $3.0 \mathrm{kHz}$. Miniature audio transducers were employed to present the stimuli to the animals. The transducers were held in a fixed spatial relation to the auditory canal by means of "pinna inserts" and leather helmets. An avoidance response in a shuttle box was used as the dependent variable. The animals' task involved the detection of a right-left reversal in a gated sequence of tone bursts. The cat appears to be about as sensitive as the human to both interaural intensive and temporal disparities at each of the frequencies studied. [This research was conducted at Indiana University, Bloomington, Indiana.]

X8. Binaural Processing in the Cat. II. Detection. Donald E. Robinson and Onslow S. Wakeford, Department of Psychology, Indiana Universily, Bloomington, Indiana 47401.Detection of diotic and dichotic tones partially masked by noise was studied in the cat. The continuous noise masker was always diotic. The single gated tone bursts had frequencies of $0.5,1.0$, and $1.5 \mathrm{kHz}$ and were presented in phase (NOSO), $180^{\circ}$ out of phase (NOS $\pi$ ), and monaurally (NOSM). The transducers were held in a fixed spatial relation to the auditory canal by means of "pinna inserts" and leather helmets. An avoidance response in a shuttle box was used as the dependent variable. In contrast to human observers the cat displayed as large a release from masking at 1.0 and $1.5 \mathrm{kHz}$, as at 0.5 $\mathrm{kHz}$. At $1.5 \mathrm{kHz}$, the cat shows a significantly greater release from masking than the human.

X9. Loudness Enhancement with Monaural, Binaural, and Dichotic Stimulation. R. Elmasian aNd R. Galambos, Department of Psychology, University of California at San Diego, La Jolla, California 92037.- In this study of the loudness enhancement phenomenon [e.g., Galambos et al., J. Acoust. Soc. Amer. 51, 141(A) 1972; Sokolich and Zwislocki, J. Acoust. Soc. Amer. 52, 141-142(A) 1972〕, a tone burst, $T_{c}$, was followed 100 msec later by a $70-\mathrm{dB}$ tone burst, $S_{1}$. Subjects matched $S_{1}$ in loudness by varying the intensity of a tone burst, $S_{2}$, presented 1.5 sec. after $S_{1} . T_{c}, S_{1}$, and $S_{2}$ were all 20 -msec 5-kHz signals. Increasing $T_{c}$ in $10-\mathrm{dB}$ steps from 60 to $100 \mathrm{~dB}$ SPL yielded a progressively increased subjective loudness of $S_{1}$. The monaural and binaural paradigms produced greater and more reliable loudness enhancement than the dichotic. With $T_{\epsilon}$ at $100 \mathrm{~dB}$, loudness enhancements averaged over $15 \mathrm{~dB}$ for eight naive subjects tested monaurally and eight binaurally. With $T_{c}$ less intense than $S_{1}$, a small loudness decrement resulted. With $T_{c}$ set at $90 \mathrm{~dB}$ and $S_{1}$ at $40 \mathrm{~dB}$, enhancements of over $30 \mathrm{~dB}$, the largest so far observed, were regularly reported in the monaural paradigm.

X10. Monaural and Binaural Free-Field Speech Reception under Noise and Reverberation. AnNa K. NÁBĚLEK AND J. M. PicketT, Sensory Communication Research Laboratory, Gallaudel College, Washington, D. C. 20002.-Speech reception was studied in a single sound-treated room at reverberation times $T=0.3$ and $T=0.6 \mathrm{sec}$, to compare binaural and monaural reception in the presence of an impulsive noise (16 $\mathrm{imp} / \mathrm{sec}$ ) and a quasisteady noise (a babble of eight voices). The speech and noise sources were fixed at locations, relative to the listener, to give the largest advantage for binaural reception. Binaural gain is taken as the difference in speechto-noise ratios for $60 \%$ correct speech reception comparing binaural and monaural results. At $T=0.6 \mathrm{sec}$, binaural gain was $3 \mathrm{~dB}$ for both types of noise. At $T=0.3 \mathrm{sec}$ the gain was $5 \mathrm{~dB}$ for the babble and $4 \mathrm{~dB}$ for the impulsive noise. The longer reverberation caused a reduction in reception in both binaural and monaural conditions. These effects were larger for the impulsive noise and for low $\mathrm{S} / \mathrm{N}$ ratios. The largest reverberation effect reduced reception from $77 \%$ to $53 \%$ words correct. [Supported by Gallaudet College.]

X11. The influence of Timbre on Apparent Location of Sound in the Median Sagittal Plane. RoBert A. ButLer, University of Chicago, Chicago, Illinois 60637.--High-pitched tones in the median sagittal plane (MSP) appear to originate above lower-pitched tones irrespective of their actual location. But, is it pitch that governs these judgments? Data from the present experiment suggest that it is not. Pulses were presented at the same repetition rate, $200 / \mathrm{sec}$, but were filtered differently. Twelve Os were asked to identify which of five loudspeakers, $15^{\circ}$ apart, generated the stimuli. Each loudspeaker presented the sounds an equal number of times. Their positions ranged from $-30^{\circ}$ to $+30^{\circ}$ in the MSP. Those pulses whose spectral content centered about 630, 1600, and 2500 $\mathrm{Hz}$ were judged to originate low, median, and high, respectively, in the MSP, despite the sameness of their pitch and despite their actual locations. When the spectral content of the pulses centered about a still higher frequency, $6300 \mathrm{~Hz}$, Os now located their source correctly. Hence, in the MSP, timbre, not pitch, governs location judgments. Whether the judgments are correct depends on the spectral content of the sound.

X12. The Precision of a Human Underwater Sound Localization Response. S. H. Feinstein, Communication Sciences Laboralory, University of Florida, Gainesville, Florida 32601.The precision of a human underwater sound localization response was defined as the correspondence between the objective azimuth of the sound source and the azimuth indicated by a pointer controller by the subject. It was determined that the pointing response had an average error of 5.98 when the subjects pointed to a visual target located in the frontal plane. When the subjects were required to locate an acoustic target located at some point in $360^{\circ}$, the average error was $19.2^{\circ}$. Front to back (or back to front) reversals occurred at $0^{\circ}$ and $180^{\circ} \pm 45^{\circ}$ and amounted to $15.1 \%$ of the total responses. A replication of the visual target localization in $360^{\circ}$ yielded 
an average error of $6.98^{\circ}$. After 240 training trials in which the subject was allowed to visually correct his auditory localization, the average error in $360^{\circ}$ was $13.1^{\circ}$. Reversals were reduced to $7.1 \%$ of the total responses.

X13. Subliminal Underwater Sound Localization. H. Hollien, J. Kinney, S. Feinstern, and P. Hollien, Communication Sciences Laboratory, University of Florida, Gainesville, Florida 32601.-Recently Hollien ["Underwater Sound Localization in Humans," J. Acoust. Soc. Amer. 52 (1972)] suggested that individuals attempting to localize sound underwater may utilize kinesthetic cues for this purpose. In an effort to obtain data on this issue, two experiments were conducted; in both cases the divers were dressed only in bathing suits and life support equipment. In the first experiment, signals were provided at a pressure level that was approximately $10 \mathrm{~dB}$ below the divers' threshold at each of the respective frequencies tested. In the second study, signal strength was at $105 \mathrm{~dB}$ SPL ( $r e 0.0002 \mathrm{dyn} / \mathrm{cm}^{2}$ ), but it was masked out by a sawtooth signal delivered to the divers' auditory mechanism via a set of underwater earphones held against their heads by a neoprene hood. Data from both investigations suggest that underwater sound localization is an auditory function, as there was no evidence that any information was obtained by tactile sensation.

* Also with Naval Submarine Medical Center, New London, Conn.

Thursday, 30 November 1972

Royal Room, 9:00 A.M.

\title{
Session Y. Underwater Acoustics IV : Signal Processing
}

\author{
A. J. VAN WOERrom, Chairman \\ New London Laboralory, Naval Underwater Systems Center, \\ New London, Connecticut 06320
}

\section{Contributed Papers (12 minutes)}

\begin{abstract}
Y1. Small Signal Suppression in a Class of Optimal Adaptive Beamformers. R. S. VARGA, Bell Laboratories, W'hippany, New Jersey 07981. - Optimal adaptive beamformers are of ten designed to minimize the beam noise response while maintaining a fixed response to an incoming signal. In the real world, improvements in detection performance that could result from noise rejection may not be realized because of a signal suppression effect. In this paper, analysis of a particular adaptive beamformer (ABF) shows the signal suppression effect to be related to the reception of signals whose wavefront departs from an assumed nominal wavefront (usually planar). Experimental results demonstrate that slight distortions in the wavefront of weak signals can cause significant degradation. Moreover, the degradation in signal response for the $A B F$ is uniformly larger than that of the delay and sum beamformer (DSBF), for a given amount of wavefront distortion. In addition, ABF signal degradation is found to be not only dependent upon the amount of wavefront distortion, but upon the background noise field as well. This result is specific to the ABF and not the DSBF. In particular, ABF signal suppression is shown to increase as the noise field anisotropy increases. Thus when the conditions for ABF noise improvement are best, the potential for ABF signal degradation is greatest.
\end{abstract}

Y2. Influence of Input Quantizer Step Sizes on the Processing Gain of Digital Beamformers. H. S. C. WANG, Eda Corporation, College Point, New York 11356.-Utilizing a formulation of output power of a digital beamformer with input quantizers of unequal input and output step sizes reported elsewhere [H. S. C. Wang, Quantizer Functions and Their Use in the Analyses of Digital Beamformer Performance (to be published); also see J. Acoust. Soc. Amer. 51, 135(A) (1972)], the optimum quantizer step sizes that yield maximum processing gain are searched by the method of "steepest ascent" with a digital computer. For the case of 2 bits/channel quantization with small input $S / N(<-10 \mathrm{~dB})$, the array gain surface as a function of two independent step variables exhibits a maximum that is somewhat greater than the maxi- mum array gain achievable with uniform input quantizers. For larger input $S / N(>-10 \mathrm{~dB})$, no maximum can be found, and, as the input $S / N$ exceeds $-4 \mathrm{~dB}$, the array gain increases monotonically with respect to both input and output step variables. These phenomena were clarified by a numerical study of a "3-level quantizer" as a limiting case. The large theoretical processing gain at large step sizes is attributed to a threshold effect of the input quantizers.

Y3. Curvature Effects on Side and Aliasing Lobes of Transparent Arrays. P. W. Smith, Jr., and E. F. Berkman, Bolt Beranek and Newman Inc., Cambridge, Massachusetts 02138.A transparent array, either continuous or discrete, is located on a spherical surface. Its sensitivity is spatially constant in magnitude and phase, compensated for maximum signal in the direction of the main lobe. A stationary-phase analysis shows that a sufficiently large continuous array has large sidelobes in a direction dependent on array size. A discrete array has aliasing lobes (grating plateaux) at a lower frequency than does a similar flat array.

Y4. Information Derivable from the Doppler Polar Profile Spectrum. Ronald L. Spooner and Jude R. Nitsche, Bolt Beranek and Newman Inc., Arlinglon, Virginia 22209.There has been considerable interest recently in the use of Doppler shifted signal information as a technique for passive acoustic localization. An investigation of this phenomenon using conventional techniques, however, rapidly becomes complicated by the large number of parameters and geometrical configurations that must be considered. In particular, it is especially difficult to determine the sensitivity of the technique to measurement errors. As an alternative, this paper treats the Doppler profile in terms of its inherent information content and indicates how such information may be utilized to obtain both qualitative and quantitative results. By invoking Fourier transform and sampling techniques, the limitations of Doppler localization are viewed in terms of conventional filter bandwidth and aliasing problems. Furthermore, measurement sensitivity is treated as a generalized $\mathrm{S} / \mathrm{N}$ ratio enhancement problem. Utilizing the methods of 
this paper, a more generalized and complete understanding of the Doppler phenomena is achieved when compared to the specialized results of conventional methods.

Y5. A Technique to Verify Statistical Performance Requirements for Sonar Detection Systems. R. J. BuRatTi AND G. E. Trezona, Federal Systems Division, IBM Corporation, Onwego, New York 13827.-An automated forced-choice method of evaluating detection performance is employed instead of the familiar fixed-threshold technique. The latter method utilizes a number of preselected, constant thresholds to estimate both detection and false alarm probabilities, whereas, the former incorporates a variable threshold with a precise false alarm probability to estimate only the detection probability. The estimate is obtained by selecting the "greatest of" a number of alternatives-one containing signal plus noise and the remainder containing noise only-to determine the number of correct detections (selection of signal plus noise alternative). The advantages of the forced-choice technique are predetermined false alarm rate, a single parameter test, less required test time, and real time monitoring of test results by minimizing the amount of data reductions. A comparison of both techniques is presented for two sets of typical statistics encountered under test conditions. Because of the uncertainty associated with the variable threshold, the forced-choice results are slightly pessimistic, but are within $0.3 \mathrm{~dB}$ of the fixed-threshold results. However, the forced-choice technique can easily be made equivalent to the fixed-threshold procedure by incorporating a correction factor into the test procedure. [Work supported by Naval Ship Systems Command.]

Y6. The Principles Involved in Choosing an Explosive Source and Processing Filter for the Measurement of Transmission Loss. J. S. Hanna ANd B. E. PARK1Ns, Bell Telephone Laboratories, Whippany, New Jersey 07981.-Explosives are of ten used as a source of acoustic energy in transmission measurements in the ocean because of the broad-band spectra of underwater explosions and the wide range of levels that can be generated. At the same time, this broad-band spectrum can complicate a transmission loss measurement because the spectral character of the shock wave and subsequent bubble pulses of the shot can be very similar to the spectral character of the loss that is due to the multipath structure of the propagation. This complication can be avoided by the proper choice of the detonation depth and the charge weight. When these choices have been made, the properties of the analyzing filter of the data processing system must then be selected; this must be done with regard for the nature of that portion of the multipath structure to be resolved as well as the nature of the charge spectrum. This paper discusses the principles involved in making these choices and gives illustrations of both short- and long-range measurements.

Y7. Certain Digital Quadrature Techniques for Bandlimited Signals. O. D. Grace, Naval Undersea Center, San Diego, California 92132.-Practical methods of signal processing of bandpass signals by digital quadrature methods are discussed. These methods assume a sufficiently dense uniform sampling of a bandpass signal and include extractions of quadrature samplings from uniform samplings; polynomial interpolations, in which the sampling frequencies $f .>\pi W$ ensure cxact interpolation; and error corrections for interpolations over a finite data base. Also included are applications to the problems of measuring the instantaneous envelope, phase, and frequency of bandpass signals.

\section{Y8. Abstract withdrawn.}

Y9. Effects of Ideal Clipping on Signal to Noise Ratios-A Comparison with Linear Systems. P. R. HaRIHARAN AND R. J. Scotr, The Magnavox Company, Fort Wayne, Indiana 46804.-Numerous articles have been published that show there is a $\mathrm{S} / \mathrm{N}$ ratio loss of $1.17 \mathrm{~dB}$ when sinusoidal signal and narrow-band noise, with low input $S / N$ ratio, is passed through a hard limiter. In this paper, we analyze the effects of wideband noise on signals due to clipping. It is shown that the resulting loss in $S / N$ ratio depends not only upon the shape of the input noise spectrum but also on the location of the signal. Results are presented for real world spectra as measured in the ocean, and it is shown that for certain kinds of spectra the loss could be as high as $10 \mathrm{~dB}$.

Y10. Covariance Structure of Surface Reverberation. RoBERT L. Swarts, Honeywell Inc., Marine Systems Center, 5303 Shilshole Avenue N.W., Seattle, Washington 98107.-An analytical expression for the covariance function of underwater sea surface reverberation is obtained from the FOM model. Certain powerful results based on the properties of Fourier transforms are demonstrated. The expression is then evaluated by computer for several hypothetical Doppler density functions. Plots of the resulting covariance functions are compared with functions obtained empirically from experiments at $28 \mathrm{kHz}$ in Puget Sound, Washington. Ensemble averaging techniques are used. Effects of data nonstationarity are also illustrated.

Y11. Characterizing Volume Reverberation Signals Using Level Crossing Information. R. C. SPINDEL AND P. T. MCElroY, Department of Ocean Engineering, Woods Hole Oceanographic Institution, Woods Hole, Massachusetts 02543.-A point scatterer model is used to formulate expressions for the expected number of level and zero crossings of backscattered volume reverberation signals. It is shown that zero crossing measurements on narrow-band transmitted signals can yield quantitative estimates of scatterer population density in oceanic deep scattering layers. Level crossing data can be used to estimate scattering strengths. For wide-band transmitted signals, level crossing information can provide a relationship between scatterer $Q$ and resonant frequency. Some features of using crossing data are (a) level and zero crossing systems are relatively simple to implement; (b) scattering strength measurements are independent of transmitted signal waveshape; and (c) since zero crossings are unaffected by the general time dependent decrease in reverberation level, zero crossing measurement systems do not require time-varying gain compensation. An experiment east of Bermuda compares scattering strength determination through level crossings to more conventional measurements. Also, the experiment gives scatterer density as a function of depth through a scattering layer. Peak densities of between $10^{-3}$ and $2 \times 10^{-2}$ scatterers $/ \mathrm{m}^{3}$ are found. 


\title{
Session Z. Shock and Vibration III : A Review of Optical Techniques for Studying Mechanical Vibrations
}

\author{
Frank A. Andrews, Chairman \\ School of Engineering and Architecture, Catholic University of America, \\ Washington, D. C. 20017 \\ Invited Papers (25 minutes)
}

\begin{abstract}
Z1. Vibration Measurement by Laser Interferometry: Homodyne and Heterodyne Techniques. F. J. Egerhardt, Electrical Engineering Depariment, U. S. Naval Academy, Annapolis, Maryland 21402.-The theory of typical homodyne and heterodyne laser interferometers for vibration amplitude measurements is reviewed. For the former, methods of absolute measurement by analysis of the harmonic series with Bessel coefficient amplitudes is presented. These methods are applicable for measurements from less than $1 \AA$ up. Limitations and noise problems are discussed. For the typical heterodyne system the detection technique and calibration required are discussed in detail. A description is given of the noise and bandwidth limitations on the range of amplitude and types of waveforms to be measured. Advantages and limitations of the two techniques are discussed in relation to each other and to other optical approaches to vibration measurement. Applications that have already been found or proposed, both for air and underwater measurement, are pointed out.
\end{abstract}

Z2. Focused Optical Imaging Systems for Vibration Measurements. J. A. CLARK, Acoustics Group, The Catholic University of America, IYashinglon, D.C. 20017.- Images of a vibrating surface can be formed by focused (lens type) scanning, and holographic (lensless) optical systems. In this paper, methods for adapting focused optical systems to obtain vibration measurements are reviewed. Included in the review are methods for determining the state of vibration from displacement, slope, and velocity measurements. The use of optical, spatial, and temporal filters, as well as reference beam interferometers is described. In particular, recent advances in the design and application of confocal mirror interferometers to vibration measurements are described. Real-time and time-averaging variations of each method are noted. The types of data obtained with each method and procedures for interpreting the data are discussed. Characteristics of the various methods such as sensitivity, cost, and convenience for in-air and in-water measurements are summarized.

Z3. Applications of Optical Holographic Interferometry in Acoustics Measurements. D. CAIN, C. Johnson, ANd G. MAYER, Naval Underwater Systems Center, New London, Connecticut 06320.- Holographic techniques are presently in wide use as an instrumentation tool in the area of vibration analysis. This paper outlines the basic concepts of holography and reviews several state-of-the-art holographic techniques that are being used or could be used for research tools and data aquisition in acoustics. The advantages and disadvantages of time-average, real-time, double-pulsed, stroboscopic, and temporarily modulated holographic interferometry and speckle interferometry are discussed. The various holographic techniques are compared with more conventional vibration measurement methods with regard to cost, accuracy, completeness of data, and range of applicability. In conclusion, several potential applications in acoustics research are presented for consideration.

\section{Panel Session (25 minutes)}

\section{Contributed Papers (12 minutes)}

\section{Z4. Abstract withdrawn.}

Z5. Electromagnetically Generated Elastic Stress Waves in Rods and Pipes. Basil P. Leftheris, Research Department, Grumman Aerospace Corporation, Bethpage, New York 11714, and P. C. Y. Lee and A. S. Cakmak, Departmenl of Civil Engineering, Princelon University, Princelon, New Jersey 08540. - A convenient laboratory method for producing highamplitude elastic stress waves in rods and pipes is described and compared with existing impact methods [C. S. Yang and R. J. Hassett, "Transient Stresses in Axisymmetric 
Bodies of Varying Area," Exp. Mech. 12, No. 7 (July 1972)]. The method employs electromagnetic repulsion of good electrical conductors to convert electromagnetic energy to mechanical energy stored in the elastic stress wave. A description of the apparatus and its design features is given as well as equations that predict the amplitude and duration of the elastic waves. The apparatus was used to study the stress wave characteristics of pipes. In particular, a stress wave with speed of propagation higher than the longitudinal phase velocity in elastic isotropic solids predicted by Chang, Cakmak, and Lee [K. P. Chang, P. C. Y. Lee, and A. S. Cakmak, "Propagation of Axially Symmetric Waves in Hollow Elastic Circular Cylinders Subjected to a Step-Function Loading," J. Acoust. Soc. Amer. 49 (1971)] was found experimentally using the new method.

\title{
26. Abstract withdrawn.
}

Session AA. Noise Propagation

\author{
RICHARD H. LYON, Chairman \\ Department of Mechanical Engineering, Massachusetts Institute of Technology, \\ Cambridge, Massachusetts 02139 \\ Invited Papers (30 minutes)
}

\begin{abstract}
AA1. Noise Propagation in the Open Atmosphere. J. E. PIEkCy, Physics Division, National Research Council, Ottawa, Canada, KIA OS1.- This contribution to the review of our present understanding of outdoor noise propagation, and the way in which it may be brought to bear on current problems, will include all effects other than those due to the presence of the ground or other surfaces. Included will be geometric spreading caused by source configuration, as well as absorption and refraction by the atmosphere. The fluctuations of sound level due to atmospheric conditions will also be discussed. The importance of bringing into engineering use the knowledge obtained in recent years in related fields such as meteorology, propagation of light in the atmosphere, and vibrational relaxations in gases will be emphasized.
\end{abstract}

AA2. Noise Reduction by Barriers. U. J. KURze, Battelle Institute, Germany.-A literature review is prepared to summarize the present state of knowledge concerning the field performance of barriers, with particular reference to screen-type barriers on finite impedance ground, to barriers of finite thickness, to right-angle wedges, to multiple barriers, and to elfects of wind. The attention is focused on practicabilities and limitations of prediction schemes applied for the design of barriers, which shall reduce the noise outdoors from vehicles on the ground and from stationary installations.

AA3. Sound Propagation over Open Terrain from a Source near the Ground-A Brief Review, Louis C. Sutherland, Wyle Laboratories, 128 Maryland Street, El Segundo, California 90245.-The noise field generated by a source near the ground can be significantly influenced by reflection and/or absorption by this ground surface. The measurement and prediction of outdoor noise sources must include consideration of this influence. Practical situations where this influence can be significant are categorized. The range of acoustic characteristics of ground surfaces are considered and the corresponding range of ground-to-ground propagation effects reviewed for steady-state noise sources near flat ground surfaces with and without various types of common ground cover. Both theoretical and empirical models for prediction of ground-to-ground propagation effects are considered. Shortcomings in the current state-of-the art are reviewed in order to suggest areas requiring further research.

AA4. Reverberation for Multipath Propagation in Urban Areas. Richard H. L.yon, Massachusetts Institute of Technology, Cambridge, Massachusetts 02139.-The propagation of urban noise combines the transmission phenomena of reflection, scattering, and shielding to produce sound levels that are substantially different from those that would exist in an open terrain situation. When the source is 
elevated, the number of important contributing paths is generally less than four or five, and the reverberation phenomenon is simply a combination of these. For street level sources, corridor propagation is dominant, complicated by the scattering that occurs on the building faces. Sound level patterns in streets, therefore, have significantly different forms than the propagation in a smooth corridor as several field experiments have shown. This paper plays a series of results from both experimental and territorial studies that demonstrate the phenomenon involved in urban propagation in addition to some of the unsolved problems that are apparent from the results.

\title{
Contributed Papers (10 minutes)
}

AA5. Effect of the Ground on near Horizontal Sound Propagation. J. E. Piercy, T. F. W. Embleton, and N. Olson, Physics Division, National Research Council, Outawa, Canada, KIA OS1.-The propagation of sound from a point source over short grass on flat ground has been measured for the relatively small grazing angles $\left(<20^{\circ}\right)$ normally encountered in practice. The distances ranged from 1 to $1000 \mathrm{ft}$ at several locations. Shadow zones caused by the ground impedance have been found in situations of importance to community noise problems. With the microphone $4 \mathrm{ft}$ above the ground and $50 \mathrm{ft}$ away from a source $1 \mathrm{ft}$ above the ground, a configuration relevant to snowmobile legislation and also exhaust noise from vehicles in suburban settings, attenuation was $\approx 15 \mathrm{~dB}$ in excess of inverse square law for frequencies near $1 \mathrm{kHz}$. For longer distances, up to $30 \mathrm{~dB}$ of excess attenuation was observed at frequencies above $500 \mathrm{~Hz}$. Two methods have been developed for determining the ground impedance at glancing angles. From these values, the attenuation in the shadow zones may be predicted using the theory developed by Rudnick (1947) and Ingard (1951). It is found that the concepts of ground and sky waves used for electromagnetic propagation are more helpful in predicting and understanding these phenomena than the inverse square law currently used for acoustic problems.

AA6. Atmospheric Ray Tracing. B. G. Roserts, JR., AND E. H. BebBs, Acoustical Division, Naval Research Laboratory, Washington, D. C. 20390.-A study that has received added consideration in the past few years is the propagation of sound in the atmosphere. This was occasioned by the introduction of jet aircraft and the resulting noise which required locating airports in such a position so as to minimize the undersirable effects. A study of the acoustical ray paths in the atmosphere would provide criteria for selecting an appropriate location.
A computer program has been written for the CDC-3800 computer to predict the acoustical ray paths in the atmosphere, as well to determine the transmission loss in air. The program accepts data in the form of a temperature or sound speed versus height profile. $A$ wind velocity profile may also be entered. The program will produce a printplot of the ray trajectories, as well as a printplot of the transmis. sion loss. A CALCOMP plot of the ray trajectories may also be obtained. By observing the locations where the rays strike the surrounding terrain and the resulting transmission losses, a suitable location can be obtained to produce the least annoyance.

AA7. A Climatology of Distant Airblast Propagations. JACK W. Reen, Sandia Laboratories, Albuquerque, New Mexico 87115.-Microbarograph data from Nevada atmospheric nuclear tests of 1951-1962 and some recent large chemical explosives tests are presented to show climatological patterns for long range propagation to about $225 \mathrm{~km}$. Amplitudes are normalized to 1-kiloton yield, free airburst, and corrected for height-of-burst effects. Propagations under early morning temperature inversions of ten showed double the amplitudes expected for standard hemispherical explosion wave expansion at $30-\mathrm{km}$ ranges. With sound velocity-height gradients, propagations may be reduced to $0.2 \times$ of standard amplitude at $10-$ to $30-\mathrm{km}$ ranges. Strong upper winds, at 6 - to $10-\mathrm{km}$ altitudes, occasionally gave as much as $5 \times$ magnification at $50-150 \mathrm{~km}$. Ducting by the high stratosphere, near $50-\mathrm{km}$ altitude, is seasonally directed eastward in winter, westward in summer. Amplitudes in the sound ring, near $225-\mathrm{km}$ range show as much as $3 \times$ magnification downwind and $0.006 \times$ reduction upwind. [This work was supported by the U. S. Atomic Energy Commission.]

Thursday, 30 November 1972

El Dorado W'est, 9:00 A.M.

\section{Session BB. Physical Acoustics IV : Ultrasonic Energy and Power Measurements III}

\author{
William D. O'Brien, JR., Chairman \\ Bureau of Radiological Health, Rockville, Maryland 20852
}

\section{Contributed Papers (12 minutes)}

BB1. Mapping of Ultrasonic Fields with Conventional Light Diffraction. BILL D. CoOK, Cullen College of Engineering, University of Houston. Houston, Texas 77004.-A theory is proposed from which a cross-sectional mapping of a progressive beam can be obtained by conventional light diffraction techniques, i.e., by passing a parallel beam of light through the sound field. To obtain sufficient information, the sound field must be rotated around its major axis for a series of optical measurements. These measurements, along a line perpendicular to the sound beam axis, are to yield the strength and phase of the effective phase grating. As an extensive amount of data is needed to compute one map ultiliz- ing two-dimensional Fourier transforms, a data acquisition system and digital computer are required. However, in the constraint of linear acoustics, one set of measurements will permit the calculation of the sound field in all space.

BB2. Absolute Determination of Acoustic Intensity by the Method of Radiation Force on a Solid Elastic Sphere. A. J. Averbuch, F. J. Fry, and F. DUnN, Bioacoustics Research Laboratory, University of Illinois, Urbana, Illinois 61820.Hasegawa and Yosioka [J. Acoust. Soc. Amer. 46, 1139 (1969)] have modified King's theory [Proc. Roy. Soc. A137, 212 (1935)] of acoustic radiation force on a solid sphere sus- 
pended freely in an inviscid fluid in a plane progressive sound field to include the elastic properties of the solid. Evaluation of the acoustic radiation force function $\gamma_{p}\left(=F / \pi a^{2} E\right)$, where $F$ is the radiation force on the sphere of radius $a$ and $E$ is the mean energy density in the plane progressive sound field, has been extended to cover the range of values of $k a(k$, the wavenumber) from 0 to 50 for type 440 stainless-steel ball bearings and water, and compared with experimental studies in which wavelength and radius of the sphere have been varied.

BB3. Scattering-Matrix Description and Nearfield Measurements of Electroacoustic Transducers. D. M. KERNS, Electromagnetics Division, National Bureau of Standards, Boulder, Colorado 80302.--Recently developed analytical techniques for the measurement of microwave antennas at reduced distances are "translated" into corresponding techniques for the measurement of electroacoustic transducers in fluids. The basic theory is formulated in scattering-matrix form and emphasizes the use of plane-wave spectra for the representation of sound fields. This theory, in contrast to those based on asymptotic description of transducer characteristics, is suitable for the formulation and solution of problems involving interactions at arbitrary distances. Two new techniques (in particular) are described: One, utilizing deconvolution of transverse scanning data, which may be taken at distances $d$ much less than the Rayleigh distance $d_{R}\left(=a^{1} / 2 \lambda\right)$, provides a means of obtaining complete effective directivity functions, corrected for the effects of the measuring transducer. Applicability of a (two-dimensional, spatial) sampling theorem and the "fast Fourier transform" algorithm, which greatly facilitate the necessary computations, is shown. The second technique provides a means of extrapolating received signal as a function of distance (observed with $d \sim d_{R}$ or $d \gg a$ instead of the conventional $d \sim d_{k}$ ) to obtain on-axis values of effective directivity. Other possible applications are indicated. In these techniques, pressure data are rigorously sufficient; normal velocity data are not required.

BB4. Generalized or Adjoint Reciprocity Relations for Electroacoustic Transducers. A. D. YAGHJIAN, NRC Postdoctoral Research Assaciate, National Bureau of Standards, Boulder, Colorado 80302.--The "equations of motion" of a linear electroacoustic transducer are written in the alternative forms $L^{+} X=0, L^{-} X=0$, where the matrices $L^{ \pm}$are linear differential expressions, $X=(E, H, u)$ represents electroacoustic fields, and the superscripts distinguish selected normalizations of the equations of motion. To each operator $L^{ \pm}$corresponds a mathematically defined adjoint operator $\left(L^{ \pm}\right)^{a}$ and an associated adjoint transducer, whose material tensor parameters are given by certain transpositions and interchanges of the parameters of the given transducer. Dissipative characteristics (lossiness, losslessness, or "gaininess") of the material of the given transducer are preserved pointwise in the adjoint transducers. A generalized reciprocity lemma leads to relations of reciprocal type between external properties of the given and the adjoint transducers. In the self-adjoint cases, the conventional electroacoustic reciprocity and antireciprocity relations are obtained and the derivation of those relations is critically confirmed. The generalized or adjoint reciprocity relations have been applied in the plane wave scatteringmatrix formalism developed for electroacoustic transducers by Kerns (Abstract BB3). Corollaries of the adjoint reciprocity relations, analogous to conventional reciprocity theorems, but involving properties of adjoint pairs of transducers, are readily derivable.

BB5. An Ultrasonic Radiation Calorimeter. Bruce Herman AND HAROLd F. STEWART, Bureau of Radiological Heallh, Rockville, Maryland 20852.-This paper describes a constant temperature environment calorimeter for the absolute measurement of ultrasonic power. This design uses a variation of the classical calorimetric procedure of power measurement as applied specifically to ultrasonic transducers. This measurement technique has advantages over other methods in that it is the least affected by beam shape and pulse duration. The calorimeter consists of a conical shaped calorimeter cup mounted in an outer brass cylindrical container. The calorimeter is placed in a water bath, which provides both a constant temperature environment and an effective medium for the propagation of ultrasound. The calorimeter cup is filled with Dow Corning 710 silicon fluid, which is an effective absorption medium. It has a $1 \mathrm{f}$-in. entrance window of gold coated nylon separating the silicon fluid from the water bath. The gold film is used as a resistance heater to maintain quasiadiabatic conditions between the silicon absorbing fluid and the water environment at the entrance membrane. The temperature difference between the silicon absorbing fluid and the outer brass cylinder is measured with a bank of chromelconstantan thermocouples. This measured temperature difference is a measure of the ultrasonic power. Calibration of the system is accomplished with a constant resistance heating coil immersed in the Dow Corning 710 absorbing fluid.

BB6. A Scanning System for the Measurement of Beam Profiles of Ultrasonic Therapeutic Transducers. JERR y HarRIS and HaRold F. StEwart, Bureau of Radiological Health, Rockville, Maryland 20852.-A system for measurement of the intensity distribution across the beams of ultrasonic therapeutic transducers is described. The system consists of a mechanical positioner, a small receiving hydrophone, electronic signal processing circuitry, and an $X-Y$ plotter. The positioner gives manual control over the location of the hydrophone within the beam. The signal from the hydrophone is fed to the electronics, which gives an output proportional to both the spatial intensity and the integrated intensity as the hydrophone is scanned across the beam. These outputs are fed to the $Y$ axis of the plotter. The $X$ axis is driven by a voltage proportional to the position of the hydrophone. The resulting plots are used to determine how the energy radiated from the therapeutic transducer is distributed within the beam.

BB7. A Portable Ultrasonic Radiometer. HAROLd F. STEwART AND Ronald RoBinson, Bureau of Radiological Health, Rockville, Maryland 20852. - The prototype of a portable ultrasonic radiometer for use as a field survey intrument of therapeutic ultrasonic equipment has been developed by the Bureau of Radiological Health. This instrument employs a solenoid balance arm principle. One end of the balance arm has a movable helical coil, which moves inside a fixed helical coil, and the other end has an airbacked target $6 \mathrm{~cm}$ in diameter, which intercepts the entire ultrasonic field. Near frictionless rotation of the balance arm is achieved using a jeweled fulcrum. A reference position of the target is predetermined as the null point, and upon application of the ultrasonic power, deflection of the target occurs. Current through the solenoid is used to reposition the target at the null point, and the current required is an indicator of the total ultrasonic power. A digital readout system directly displays this power output utilizing a manual zeroing capability. The instrument provides a wide dynamic range of $100 \mathrm{~mW}$ $100 \mathrm{~W}$ with a reproducibility error of less than $\pm 10 \%$. The advantages of the resulting system over other measuring systerns such as the chemical microbalance, radiation pressure float system, hydrophone, calorimeter, etc., are portability, ease of measurement, digital readout, manual zeroing capability, direct linear measurement method, closed system, ease in setup, and comparable accuracy and reproducibility.

BB8. Technique for Measuring Ultrasonic Power. J. G. ZIEDONIS, Hoffmann-La Rache Inc., Rache Medical Electronics Division, Cranbury, New Jersey 08512 . - - It is vitally important 
to know accurately the ultrasonic energy levels of electronic equipment that generates and sends ultrasonic energy into the human body. Therefore, a radiation force measuring technique was developed to measure the ultrasonic energy generated by such equipment. Ultrasonic energy levels of less than $1 \mathrm{~mW} / \mathrm{cm}^{2}$ have been measured, with good repeatability. The equipment used is a Mettler Balance, model $\mathrm{H}-20-\mathrm{T}$, with a readable sensitivity of $0.01 \mathrm{mg}$ and a target that detects the incident radiation force. The target is attached to the balance weighing pan and suspended in water under the balance by a nylon line. The balance has a built-in tare, used to counteract the ultrasonic energy force that illuminates the target. In addition the tare is adjusted to counteract the surface tension that normally occurs at the nylon support line, which would interfere with the accuracy of power measurements. The target consists of an air-backed reflector that has a half angle of $45^{\circ}$. This angle and the air space behind the thin metal surface makes the target a nearly perfect reflector. To make certain that no ultrasonic energy is reflected from the walls of the water tank, the ends of the tank are tilted about $20^{\circ}$, and all the inside surfaces are lined with ultrasonic energy absorbing material. This equipment has been in use now for over a year. Ultrasonic energy power measurements have been made on many different sizes, configurations, and frequencies of ultrasonic transducers. Details of this technique of measuring ultrasonic power will be reviewed in the paper.

BB9. Ultrasonic Field Measurements for Flaw Analysis. Joseph L. Rose and Paul A. MEyer, Drexel Universily, Philadelphia, Pennsylvania 19104.-The purpose of this paper is to introduce experimental sonic field measurement procedures that can be used to analyze ultrasonic transducer wave generation characteristics and wave reflection patterns from specially shaped flaws in solid materials. This work is essential for understanding principles of ultrasonic nondestructive evaluation in homogeneous isotropic materials as well as advanced composites and multilayered bonded structures. Very little work to date has been done on this subject except for the work related to the classical flat bottom hole equivalent that is so widely used in ultrasonic inspection test standards today. It is shown in the paper that sonic field pressure profiles obtained in both pulse-echo and throughtransmission modes can be used to characterize flaw shapes, sizes, and locations. The following details are included: (1) Experimentally obtained pressure profiles in solid blocks are compared with results obtained from a theoretical solution for wave propagation in a solid material. The effects of transducer frequency, diameter, and type on the sonic field pressure profile are discussed. (2) Experimental ultrasonic field pressure profile measurements are compared with results obtained from a finite difference two-dimensional computer code for elastic wave propagation across a rectangular flaw in a solid block. Applications of the computer code to other problems in flaw detection are presented. (3) Ultrasonic pressure profiles are presented for wave reflection patterns across such flaws as cracks and elliptically and circularly shaped holes. Guidelines for establishing inspection standards based on this work are presented.

BB10. A Differential Microcalorimeter for the Measurement of Ultrasonic Powers at the Milliwatt Levels. Adnan Sokollu and Serpil Guvenc, Case Western Reserve Universily, Cleveland, Ohio 44106.-A microcalorimeter system has been developed to evaluate ultrasonic power outputs, especially of pulsed diagnostic transducers. The system is composed of two basically identical calorimeters, interwoven by an extensive network of thermocouples serving as an averaging temperature comparator. Borh calorimeters are mounted into a common housing with heavy aluminum walls so geometrically as to ensure a well-balanced thermal equilibrium. Either calorimeter may be used as an energy recipient, while the other serves as a temperature reference. Their filling consists of a silicon oil that matches the acoustical impedance of water. Each calorimeter contains a heating wire for calibration and a cooling system to achieve a fast temperature equilibrium following the measurement. The thermocouple battery is formed by 30 pairs of junctions, made of 20 - $\mu$-diam copper and constantan wire. It permits temperature evaluations at the millicentigrade level. The overall sensitivity of the system is approximately $0.08^{\circ} \mathrm{C}$ per calorie. Transducers to be measured are inserted into one of the calorimeters and are energized for about $30 \mathrm{sec}$ and then pulled out to prevent the heat created in the transducer from reaching the area of measurement. Both pulsed or CW diagnostic transducers have been measured to ultrasonic power levels as small as $1 \mathrm{~mW}$ and with an accuracy of about $\pm 4 \%$.

BB11. Self-Reciprocity Calibration of Echo Ranging Transducers. John M. ReId, Providence Hospilal, Sealtle, Washington 98122. - The technique of reciprocity calibration, which allows the absolute determination of sound quantitaties, has been effectively used for many years at low frequencies. The self-reciprocity modification of this technique requires fewer measurements and has higher accuracy. Present day pulse echo systems have been used to perform this calibration on their own transducer. These transducers are available with wide bandwidths and can be used to measure both peak and average fields. The technique is discussed from the standpoint of energy density, from which the self-reciprocity conditions and derived in a convenient form. The choice of auxiliary measurements of impedance or open-circuit voltage is also discussed. No acoustical standards ane required. The technique appears to be suitable for average power measurements on pulsed and CW systems. Corrections for short-pulse operation of typical medical systems require further investigation.

Thursday, 30 November 1972

El Dorado East, 9:00 A.m.

Session CC. Engineering Acoustics III : Transducers and Ingenious Devices

HARRY B. Miller, Chairman

Naval Underwaler Systems Center, New London, Connecticut 06320

Invited Paper (30 minutes)

CC1. High-Performance Magnetostrictive Transducers. A. P. Edson and E. L. Huston, International

Nickel Co., Inc., Paul D. Merica Laboratory, Sterling Forest, New York 10901.--This paper is directed 
to the use of magnetostrictive materials for the generation of high-intensity mechanical vibration, sound, and ultrasound. It will present a mathematical analysis of a novel method of electromagnetic polarization and excitation that affords previously unattainable combinations of peak-to-peak magnetostrictive strain energy, electromechanical efficiency, and linearity. The analysis is based on a nonlinear model valid for isotropic, square-law magnetostrictive materials. To achieve these results, electromagnetic excitation is applied at a large angle relative to the axes of static polarization and mechanical output. Because this is usually most conveniently achieved by application of magnetic vectors at right angles to one another, the method has been termed "orthogonal excitation." A specific system for orthogonal excitation, which permits electromagnetic self-polarization, will also be discussed.

\section{Contributed Papers (10 minutes)}

CC2. Use of Orthogonal Fields in Magnetostrictive Seroll Transducers. C. L. LeBlanc and C. H. Sherman, Nezu Landon Laboratory, Naval Underwater Systems Center, New London, Connecticut 06320.-A linear magnetostrictive theory is used to investigate the alternating small signal characteristics of a thin-wall scroll transducer polarized and driven with orthogonal magnetic fields. The concept of orthogonal excitation, advocated by researchers in the International Nickel Co., is studied from this linear viewpoint, and values for the effective electromechanical coupling coefficient are presented in terms of the material coupling coefficient $\boldsymbol{k}_{33}$ derived by applying collinear polarization and excitation magnetic fields along the axis of principal strain. Although the effective coupling was found to depend quite heavily on the magnitude and orientation of the resultant magnetic fields, no case was encountered where the effective coupling exceeded $k_{\mathrm{a} 3}$. However, it is expected that orthogonal excitation will have genuine advantages under large signal driving conditions, and two special cases of high-amplitude drive (linearized and unpolarized drive) will be discussed.

CC3. Further Experiments with Impediography: A New Ultrasonic Technique with Biomedical Implications. JoIE Pierce Jones, Bolt Beranek and Newman Inc., 50 Moulton Street, Cambridge, $\boldsymbol{A}$ assachusetts 02138.-A new technique suitable for medical diagnoses and nondestructive testing was recently introduced [Papers NN7 and NN8, 83rd Meeting, Acoust. Soc. Amer.]. Termed impediography, this method employs time-domain deconvolution of appropriately shaped acoustic impulses and their echoes to produce a temporal waveform that can be related to physical parameters such as impedance. Thus, impediography allows us to measure accurately the specific acoustic impedance at an arbitrary position within a test object. In preliminary experiments using a spark source to illuminate the test object, analysis of the reflected signal gave the impedance as a function of the acoustic travel time. This acoustic "picture" is termed an impedogram. In this paper, two particular experiments will be discussed in detail. In the first, impedograms of a soft rubber test object were obtained under various degrees of tension. Changes in impedance were clearly observed as the sample was stretched. In a second experiment, a two-dimensional impedogram was obtained of a rabbit's hind limb. This impedance profile showed excellent correlation with the actual biological structure: bone, bone marrow, skin, and even fascia were clearly distinguished.

CC4. Piezoelectric Ring and Spherical Transducers Designed to Vibrate in a Dipole Mode. John Chervenak, Acoustics Division, Naval Research Laboratory, Washington, D. C. 20390.-Free-flooding piezoelectric ring transducers have good efficiencies, are capable of radiating bigh acoustic power, are of simple design, and have the mechanical strength required for deep underwater submergence operation. However, the omnidirectional acoustic radiation in the plane of a ring transducer is undesirable for applications where directional beams are required. Directional acoustic beams may be obtained from nondirectional transducers by the use of reflectors that are bulky, costly, and difficult to maintain for large lowfrequency arrays or by using volume arrays in which the transducer elements are spaced and phased in such a manner that acoustic radiation from all transducers adds in the direction along the acoustic axis of the array and cancels in the opposite direction. Since the technique used to get directionality from biplanar or volume arrays controls acoustic radiation only along the $0^{\circ}$ and $180^{\circ}$ but not the $90^{\circ}$ and $270^{\circ}$ directions associated with beam pattern charts, the system works best when transducers with dipole (figure-eight type) beam patterns are used. The design of a dipole ring and spherical transducer will be discussed and measurement data presented.

CC5. Suppression of Transducer Sidelobes by the Use of Acoustic Baffles. B. G. WatTers, Bolt Beranek and Newman Inc., Cambridge, Massachusetts, AND G. C. GodDaRD AND V. G. WELSBY, University of Birmingham, Deparlment of Electronic and Electrical Engineering, Birmingham B15 $2 T T$, England.-The out-of-beam response of underwater acoustic transducers may be reduced by the use of baffle systems. This paper presents the results of experimental work carried out recently at the Unjversity of Birmingham (U.K.) on the effect of high-transmission-loss pressure-release planar baffles placed to the side of the main axis of a transducer. The height of the baffle (i.e., its projection beyond the transducer face) and the distance in wavelengths from the transducer axis have been varied over a wide range. Our results are compared to values calculated on the basis of Fresnel diffraction theory.

CC6. Attenuation of a Resonant Dissipative Silencer in a Rigid Duct. Thomas J. Trella, Sound Control Department, Koppers Company, Inc., Ballimore, Maryland 21230 AND Peter K. KASPER, NFK Engineering Associales Inc., Silver Spring, Maryland.-Sound attenuation characteristics of a resonant-type dissipative silencer consisting of a reactive chamber with a porous facing have been considered. Such a silencer provides a high degree of attenuation within a narrow frequency range. Predicted attenuation values are compared with experiment for plane waves propagating in a rigid duct containing the dissipative silencer. The sound field is described by one-dimensional acoustical expressions taking into account the effect of boundary conditions and the presence of the silencer. 'The theoretical model incorporates the acoustical properties of porous materials and inertance of the sound field in the duct adjacent to the silencer. Good agreement was achieved between theoretical predictions and actual measurements. Results presented indicate the dependence of the attenuation spectrum upon flow resistivity and thickness of the porous material.

CC7. Noise and Vibration Reduction in Piping Systems by Flexible Couplings. P. H. W'HITE and P. E. RENTZ, Bolt Beranek and Newman Inc., Los Angeles, California.-Unwanted noise and vibration in piping systems, generated by 
pumps, valves, or orifices can be attenuated by flexible couplings. The efficiency of the resulting noise reduction is governed by the physical characteristics of the devices and the distribution of energy between the pipe wall and the contained fluid. A good attenuator must reduce both fluid-borne and structure-borne noise. Experiments confirm the analytical prediction of energy distribution for acoustic and mechanical excitation of the system. Further experiments on various 4-in.-diam flexible couplings demonstrate their relative noise reduction characteristics for fluid-and structure-borne noise. A two-element right-angle configuration is shown to be even more than twice as efficient as one isolator, particularly at low frequencies.

CC8. Gradient Loudspeakers. Harry F. Olson, RCA Laboratories, Princeton, New Jersey 08540.-The directivity of gradient-type loudspeakers depends upon the difference in phase of the generated sound pressures, or powers of the difference in phase of the generated sound pressures, between two or more points in space. The directivity of the conventional wave-type loudspeakers depends in some manner upon wave interference of the sound emanating from the elements of the radiating surface. To obtain any semblance of directivity from the wave-type loudspeaker, the radiating surface must be comparable to the wavelength. Therefore, the dimensions of the wave-type loudspeakers become relatively large in the low audiofrequency range. In contrast, gradient-type loudspeakers are small compared to the wavelength. For sound reproduction requiring directional loudspeakers, the gradienttype loudspeaker provides the features of relatively small size and uniform directivity with respect to frequency.

CC9. The STL-Ionophone Transducer. F. J. FransSON AND E. V. Jansson, Department of Speech Communication, Royal Institule of Technology, $(K T H) S-10044$ Stockholm 70, Sweden.The STL-ionophone consists of a de discharge in air. The discharge emits sound when an ac current is superimposed on the dc current. It represents a small sound emitter with high internal impedance and constant source strength from low to ultrasound frequencies. It can also register air motions as a superimposed voltage and works in this function from zero to ultrasound frequencies. It has been employed as a sound source for measurements on woodwind instruments and models of the human vocal tract. As a receiver, it has been employed to study the sound field at the embouchure of flutes. Fundamental properties registered and applications will be discussed.

CC10. Frequency Response of Condenser Microphones in Gases other than Air. W. R. Semrau, Detroit Diesel Allison, Division of General Motors Corporation, Indianapolis, Indiana 40206.-Performance of scale model fan noise experiments in a unique free-field heavy gas test facility required that a method be developed for determining microphone response in the high molecular weight gas. Using an electrostatic actuator, calibration experiments in air, argon, helium, and freon-12 were conducted at several gas pressures. Results indicated that microphone pressure response is essentially independent of the test gas composition. At diaphragm resonance frequency, where the greatest sensitivity variation was observed, changes due to gas composition were only about $1 \mathrm{~dB}$. Apparently diaphragm damping is governed by pressure rather than gas density. This result obviates the need for microphone calibration in gases other than air.

CC11. Analysis of the Operation of Electret-Key Transducers. G. M. SEssler, Bell Laboratories, Murray Hill, New Jersey 07974.-The operation of electret-key transducers [see G. M. Sessler and J. E. West, Proc. Int. Congr. Acoust., 7th, Budapest (1971), Paper $23 \mathrm{E} 1]$ is studied analytically. In particular, the relations between generated voltage and change in transducer capacitance due to large-amplitude diaphragm displacements are derived for transducers terminated by a combination of resistive and capacitive components. Numerical results for the voltage generated by diaphragm displacements with trapezoidal time dependence are also given. Finally, a comparison of calculated and measured responses is made. With certain modifications, the present analysis applies also to other electret transducers, such as graphic electret tablets, electret line transducers, and electret vibration transmitters.

CC12. Measurement of Acoustic Impedance of Several Artificial Ears. H. D. Record, TRACOR, Inc., A ustin, Texas, AND E. L. HIxson, Department of Electrical Engineering, The University of Texas at Austin, Austin, Texas 78712.- The driving point acoustic impedance of five artificial ears was measured over the frequency range $100-7000 \mathrm{~Hz}$. An acoustic impedance measuring device was constructed, and consisted of a small pistonphone, equipped with an accelerometer within the piston and a 1 -in. insert microphone. Operational amplifier integration and summing, together with an $X Y$ recorder, allowed the continuous plotting of measured acoustic impedance as the frequency range was swept. Since acceleration and pressure were not measured at precisely the same point in the system, a computer program was used to perform the required phase correction and thus obtain true driving point impedance values. The impedance measuring device and phase correction procedure are described, and the results of the measurements are presented. Artificial ears included are the NBS-9A coupler, the ANSI type 1 coupler, the B\&K type 4153 artificial ear, a TRACOR experimental coupler, and an artificial ear constructed for this study. Measured values of acoustic impedance are compared with design values.

Thursday, 30 November 1972

Silver Chimes East, 9:00 A.M.

Session DD. Speech Communication V : Voice Frequency and Physiology

Victoria Fromkin, Chairman

Department of Linguistics, Universily of California, Los Angeles, California 90024

This is a special session; preprints provided; discussion only.

\section{Contributed Papers}

DD1. Computer Modeling of Vocal Cord Pathologies and Voice Registers. Ingo R. Titze and Will.tam J. Strong,
Department of Physics, Brigham Young University, Provo, Utah 84601.-A detailed study of normal vibrational patterns 
of the vocal cords and a preliminary investigation of simple vocal cord pathologies have been conducted. A 16-mass model described in earlier abstracts [Titze and Strong, J. Acoust. Soc. Amer. 51, 138(A) (1972); 52, 123 (A) (1972)] represents mucous membrane, vocal ligament, and vocalis muscle. An 18-section vocal tract and a 12-section nasal tract are coupled to this system. Fundamental frequency, vocal dynamics, register, and vibrato are linked quantitatively with the physiological correlates, i.e., subglottal pressure, vocalic contraction, vocal cord length, and elastic properties of vocal ligament and mucous membranc. Interactions between the vocal tract and the glottal source are demonstrated for the vowels /a/ and /i/. An attempt is made to simulate localized mass increase, i.e., a vocal node, and localized stiffening to approximate the effect of a mild cancer. The perturbed vibrational patterns and the corresponding synthesized vowels are discussed. Samples of normal utterance's and slightly pathological utterances are presented for several pitches in modal and falsetto register.

DD2. Preliminary EMG Investigation of Certain Intrinsic and Extrinsic Laryngeal Muscles in Patients with Spasmodic Dysphonia. G. N. McCall, R. H. Colton, and D. D. RABUzZ1, Department of Otorhinolaryngology, $S L^{\prime} V Y$ Upstale Medical Center, Syracuse, New York 13210.-ENG studies were conducted on eight patients with nontremor related spasmodic dysphonia. Bipolar hook-wirc electrodes were inserted into the cricothyroid, sternothyroid, and throhyoid muscles of each patient. The electromyographic activity from each muscle was recorded along with the voice of each patient on a multichannel FM data recorder for subsequent writeout and analysis. Data were obtained during quiet respiration, as well as during each patient's utterance of a standard sequence of isolated vowels, CV syllables, words, and contextual speech. Data analyses revealed that the characteristic intermittent voice stoppage episodes exhibited by patients with spasmodic dysphonia occur in association with sharp, burstlike increases in EMG activity in the cricothyroid and in some instances the sternothyroid and thyrohyoid muscles. Patients with spasmodic dysphonia tend to show prolonged contraction of the muscles under study prior to and following phonation. The postphonatory time data provide evidence suggestive of a problem in muscle relaxation. Implications of these preliminary observations will be discussed and directions for further investigation suggested.

DD3. A Study of Larynx Height in Speech Using the ThyroUmbrometer. William G. Ewan and Robert Krones, Phonology Laboratory, Department of Linguistics, University of California, Berkeley, California 94720 .-The thyro-umbrometer consists of an array of seven rectangular photocells $\frac{1}{2}$ in. $\times 1 \frac{1}{4}$ in., placed on one side of the subject's neck (arranged so the long axis of the photocells is normal to the surface of the throat) with a point light source on the other side. The shadow of the subject's thyroid prominence is cast on the photocells. The output of each photocell is fed to a small computer, which locates the laryngeal prominence at the level of that photocell producing the least voltage. Preliminary results in 1racking the larynx height and $F_{0}$ reveal that (1) as has been widely reported in the literature, larynx height and $F_{0}$ are positively correlated, but (2) larynx height is greatly affected by vowel and consonant articulation; specifically, (3) voiceless stops have consistently higher laryngeal position than phonetically similar utterances containing voiced stops. [Supported by the National Science Foundation.]

DD4. Predictors of Age in the Male Voice. W. J. Ryaiv, Department of Communicative Disorders, University of New Mexico, Albuquerque, New Mexico, AND K. I1: BURk, Insiitute of Logopedics, Wichita, Kansas.-Direct age estimates of 80 adult male speakers, chronologically 40-80 years of agc, were made by 20 untrained listeners from recorded speech samples. Forty recordings were selected for further perceptual and acoustic analy'se's based upon high listener agreement of porceived age. Judgments by trained listeners of the presence or absence of selected voice characteristics, in addition to five acoustic measurements were intercorrelated. The significance of these variables in predicting perceived age was assessed through a multiple regression analysis. Results indicated that five voice characteristics: voice tremor, laryngeal tension, air low, imprecist consonants, and slow rate of articulation, wire strong predictors of perceived age, and suggest sperific areas of future study involving alge related physiologic, acoustic, and perceptual changes in speech production.

DD5. Fundamental Frequency Characteristics of Young Deaf Adults During Oral Reading. Yosuryuki HoRII AND Milo E. Bishop, Purdue L'niversity, Lafayette, Indiana 47907.--A total of 18 young deaf adults read a 181-word passage, and distributional characteristics of fundamental frequency $\left(f_{0}\right)$ were investigated using a period-by-period $f_{0}$ analysis program. The results of analysis showed significantly higher $f_{0}$ means and inaller standard deviations and mid-90\% range than those for normal-hearing subjects. I heal specch was characterized, furthermore, by significantly less skewness of fundamental frequency distribution. In addition, bimodality or multimodality of $f_{0}$ distributions commonly observed in speech of normal-hearing subjects were also less clear in the deaf speech samples. The results indicate limited, monotonous pitch variability and almost constantly strained type of phonation in deaf speech. [This research was supported in part by Air Force Cambridge Research Laboratories, OAR, and by National Institute of Dental Research.]

DD6. Speed of Pitch Change. John J. Ohala And William G. Ewav, Phonology Laboratory, Department of Linguistics, University of California, Berkeley, California 94720 .- In an attempt to determine the response characteristics of the larynx in voluntary pitch change, five adult male subjects were instructed to execute a variety of continuous pitch changes, as rapidly as possible, within the range $90-220 \mathrm{~Hz}$. For a given pitch interval, there was a marked tendency for an upward pitch change to take longer than a downward pitch change. Also, unexpectedly, there was no marked tendency for a change involving a wide pitch interval to take longer than a change involving a smaller interval. Speculations on the physiological reasons for these relations, as well as their possible relevance to the phonology of tone and intonation, will be offered. [Supported by the National Science Foundation and a University of California Faculty Research Grant.]

DD7. Observations on a Multiple Harmonic $F_{\sigma}$-Tracking Program. T. B. Sriow and Allen Montgomeky, Purdue l'nizersity, IVest Lafayetle, Indiana 47007.-This paper presents some special features of an $F_{0}$ tracking algorithm that proceeds logically to examine the harmonic structure of speech signals. The results of the procedure are $F_{0}$ contours suitable for such purposes as intonation studies, synthesizer inputs, statistical studies of vocal frequency, etc. Characteristics of the sy'stem include a very low error rate and a conservative treatment of segments whose spectra are not clear. 'This treaument is based on continuity and whole-number ratio constraints on harmonics detected from short-time spectra. Error rates as low as one discontinuity in a 10-sec sample have been achieved, with errors generally being simple multiples of the true fundamental, and therefore easily detectable. Comments on the relationship of this approach to human litch letection will be made, and the potential application of aspects of this methor to the output of other $F_{0}$ extraction systems as a refinement procedure will be discussed. 
DD8. Intrinsic $F_{0}$ in Vowels: Physiological Correlates. J. E. AtKinson, Naval Underwater Systems Center, New London, Connecticut 06320, and Department of Linguistics, University of Connecticut, Storrs, Connecticut 06268.- - t has been known for some time that different vowels characteristically have different average fundamental frequencies $\left(F_{0}\right)$ The most influential explanations offered to account for these "intrinsic" differences in $F_{0}$ are (1) dynamogenetic radiation of muscle potentials (e.g., Taylor, 1933); (2) mechanical interaction with the supralaryngeal musculature (e.g., Lehiste, 1971); (3) aerodynamic coupling between the largynx and the oral cavities (e.g., Flanagan, 1968). In the present study, a simplified explanation of source-system interactions is given in terms of an electrical equivalent circuit. From this model several physiological parameters are predicted for different vowels. Measured physiological (air flow rate and subglottal pressure) and acoustical data are compared with these predictions. The measured data in all cases support the results predicted by the model. This study supports the Flanagan model for sourcesystem interaction and extends its scope in terms of physiological correlates.

DD9. Influences of Phonetic Sequences and Stress on Fundamental Frequency Contours of Isolated Words. WAyNE A. LEa, Univac DSD, P.O. Box 3525, St. Paul, Minnesola 55165.-At vowel onset following unvoiced consonants in /haCVC/ utterances spoken by two talkers, $F_{0}$ began high $(30 \%$ higher than in $/ \% /)$, and fell about $7 \%$ in the first 5 csec. At closure of voiced oral obstruents, $F_{0}$ suddenly dipped about $10 \%$, remained flat, suddenly rose about $25 \%$ at opening of closure, and, after vowel onset, gradually rose (an average of $8 \%$ in the first $10 \mathrm{csec}$ ). The high/low feature of the vowel and the manner and place of prevocalic consonant articulation had progressively less effect on vowel $F_{0}$ values. The final consonant had no apparent effect on $F_{0}$ contours in the vowel. As previous synthesis work has suggested, the fall or rise of $F_{0}$ in the initial portion of a vowel appears to be a cue to the state of voicing of previous consonants. Initial and peak $F_{0}$ values in the vowel also can indicate state of consonant voicing. However, $F_{0}$ contours in bisyllabic words with contrasting stress patterns and similar phonemic sequences (e.g., permit, permit) showed that (1) an initially falling $F_{0}$ in a vowel may indicate either previous unvoiced consonant or an unstressed vowel, and (2) a rising $F_{0}$ contour may indicate either a word-initial vowel, a preceding voiced consonant, or a stressed vowel.

DD10. Perception of Rhythm and Accent in English Words and Phrases. Sandra Pruzansky and Myron Wish, Bell Laboratories, Murray Hill, New Jersey 07974.-Subjects were presented pairs of three-syllable words and phrases and were asked to indicate how much the elements of a pair differed in rhythm and accent. In one session, subjects read the words aloud; dashes were placed between syllables to make the words and phrases orthographically similar. In another session subjects listened to the pairs spoken by a single speaker; there was no written presentation of the stimuli. Separate multidimensional scaling analyses of the two sets of data revealed that three of the dimensions underlying each set were basically similar, but there were some interesting differences between tasks. The three similar dimensions were related to the location of the syllable with primary stress and the number of stressed syllables. In an earlier study using the same stimulus set and judgment task, Wish had found three di- mensions closely corresponding to the present ones. Two dimensions found in the earlier study, where stimulus words had been printed without intersyllabic dashes, now appear to be based on orthographic rather than acoustical differences between stimuli.

DD11. "Tone" and Pitch Perception. D. Van Lancker and V. A. Fromkin, Department of Linguistics, UCLA, Los Angeles, California 90024.-Research has demonstrated that while many aspects of language processing are lateralized to the left cerebral hemisphere in man, perception of pitch differences is not. We have conducted experiments in the dichotic listening paradigm to determine whether speakers of tone languages show a right ear advantage in processing contrasting "tones." Three sets of stimuli were used : randomly paired combinations of five Thai words differing only in tone; paired lists of five Thai words contrasting only in initial segment; the five Thai tones hummed to contrast only in pitch level and direction. Thai speakers were used as subjects. The preliminary results show that for Thai speakers, there is a right ear preference for pitch stimuli only when the contrasting pitches represent linguistic "tones." Consonantal discrimination and "hums" were processed as expected : a right ear preference for consonants, a left ear preference for nonlinguistic pitch. [This research was supported in part by a U. S. Public Health Services (NIH) Grant, and in part by an Office of Naval Research Contract.]

DD12. Electromyographic Study of the Tones of Thai. Donna Erickson and Arthur S. Abramson, University of Connecticut, Storrs, Connecticut 06268 and Haskins Laboratories, New Haven, Connecticut 06510.- In our desire to study the effects of laryngeal muscle activity on the rather complex use of fundamental frequency changes in a tone language, we have done an electromyographic analysis of the production of the tones of Thai. We chose the Thai language because its five phonemic tones differentiate words primarily by means of characteristic $f_{0}$ contours. In the experiment, we asked four native speakers to say 45 short sentences. Included in this list were the five tones in nine contexts: the three long vowels /aa ii uu/ and the three labial consonants/b p ph/. Our findings indicate that for all four speakers, the activity of the cricothyroid muscle increases with the raising of $f_{0}$, and the activity of the strap muscles - the sternohyoid, sternothyroid, and thyrohyoid muscles-increases with the lowering of $f_{0}$. [This research was supported by the National Institute of Dental Research, NIH Grant.]

DD13. Dichotic Perception of Lerical Tone by Speakers of Cantonese and English. Peter J. Benson, Timothy S. Smith, and Linda ARreaga, Depariment of Linguistics, University of California, San Diego, La Jolla, California 92037. -Current models of dichotic listening do not predict whether linguistic word tones would be perceived more accurately by the right or the left ear. In this experiment five Cantonese words, all having the segmental structure $/ \mathrm{ma} /$ but differing in tone, were presented dichotically to two groups of Ss. One group consisted of right-handed native speakers of Cantonese, the other of right-handed native speakers of English. Ss in both groups were first trained to identify the tones using iconic tone letter symbols, and were then tested dichotically. Overall, there was a slight but nonsignificant left ear effect for both groups. In the Cantonese group, four Ss showed a left ear effect, and three showed a right ear advantage. In the English group, three $S$ s showed a slight right ear effect, three $S_{s}$ a left ear effect, and one $S$ no ear differences at all. These results suggest that individual subjects are probably using somewhat different strategies in identifying lexical tones, and would not support claims that tonal stimuli are perceived in either a linguistic (speech) or a musical mode of processing. 


\title{
Session EE. Psychological Acoustics IV : Deafness, Audiometry, and Fatigue
}

\author{
W. DIXoN WARD, Chairman
}

\author{
University of Minnesota, Minneapolis, Minnesota 55455
}

\section{Contributed Papers (10 mimutes)}

\begin{abstract}
EE1. SKAT: An Automated, Self-Administered Preschool Auditory Screening Test. R. C. BERRy, Boston University, Boston, Massachusetts 02215 , S. J. KATZ AND T. SchaEfER, Searle Medidata, Inc., Waltham, Massachusetts.-SKAT is part ol an automated, self-administered preschool screening battery for vision, IQ, and readiness. SKAT was designed to detect educationally significant hearing and discrimination problems. Sixteen CVC words were selected for familiarity to preschoolers. The words were grouped into sets of four. All words in a given set had the same vowel; consonants differed. The four pictures for a set were displayed together on a rearprojection screen. A response button was located beside each picture. Words were presented through TDH -49 earphones. To reduce testing time, conditioning was limited to naming each picture once. When testing began, the $S$ was instructed to press the button next to, e.g., "pot." A new display and new word were presented when $S$ responded, or after 6 sec. Twelve words per ear were presented at varying intensities: four each at 55, 50, and $45 \mathrm{~dB}$ SPL, randomly. Standard puretone (Maico Audiometer) screening was administered to 61 Ss, $K-2$. Each S self-administered SKAT in $3.5 \mathrm{~min}$, average time. Six Ss $(10 \%)$ lailed both procedures. Three $(5 \%)$ failed SKAT, but passed pure tone. In similar studies, more false negatives and false positives have been reported for the VASC and Fading Numbers Test.
\end{abstract}

EE2. Conventional and High-Frequency Hearing of Aviators as a Function of Flight Time and Type Aircraft. JoHN L. FletChER, Department of Psychology, Memphis State University, Remphis, Tennessee 38111.-Conventional (500-6000 $\mathrm{Hz}$ ) and high-frequency audiograms (4000-18000 Hz) were obtained from aviators flying jet (prop or pure) fixed wing and from others piloting rotary wing (helicopter) craft. These pilots varied widely within the three categories of aircraft, i.e., jet, prop, or rotary, in the number of hours logged in the aircraft. Data were therefore analyzed in terms of type aircraft flown and exposure category (based on number of hours flown). Results indicate a progressive decline of hearing level with number of hours flown, with the decline first detected at the frequencies above the conventional range, gradually progressing with continued exposure into the conventional (and therefore speech) range. Results are discussed in terms of the relative hazard of various types of aircraft, and in terms of the importance of tests of high-frequency hearing to hearing conservation. [Supported by the Office of Naval Research, Engineering Psychology Programs, Work Unit NR 197-002.]

EE3. Human Auditory Thresholds During Deep, Saturation Helium-Oxygen Dives. W. G. Tномаs, Department of Surgery, University of North Carolina School of Medicine, Chapel Hill, North Carolina 27514.-Pure-tone air conduction thresholds and Sensory Acuity Levels were measured on 33 different divers during eight saturation dives ranging from 300 to $1000 \mathrm{ft}$ in a hyperbaric helium-oxygen environment. A total of 366 audiograms were performed at 26 different depths between surface and $1000 \mathrm{ft}$, as well as pre- and postdive audiograms at surface. All divers showed a progressive conductive hearing loss related to depth, reaching a maximum of around
$30 \mathrm{~dB}$ at $1000 \mathrm{ft}$. The greatest hearing loss occurred in the low frequencies during the first $100 \mathrm{ft}$ of the dives, with smaller increases with additional depth. During decompression, hearing levels were slower to return, with significant amounts of hearing loss remaining at 2 ft from surface. Hearing for the higher frequencies, especially $6000 \mathrm{~Hz}$, became better than surface values as surface was approached. All thresholds returned to predive levels upon reaching surface. No change was noted in the SAL for any depth.

EE4. A Discrimination Test for Nonspeech Signals. Gordon R. Bienvenue, Environmental Acoustics Laboratory, The Pennsylvania State University, University Park, Pennsylvania 16802.-Coal miners, while they are exposed to excessively high levels of noise, must be able to listen for roof talk or sounds created by stress release in the mine roof, which is indicative of an imminent cave-in. Any device that interferes with the ability of the miner to hear these roof-talk signals would constitute a safety hazard to the miner. It has been suggested that the use of ear protectors might constitute such a hazard. A set of roof-talk tapes has been assembled to assess this possible hazard. These consist of one listening tape for training subjects in listening to roof talk and a set of test tapes for testing subjects' abilities to identify the presence of roof-talk warning signals in the presence of varying amounts of noise. Six subjects were tested using these roof-talk test tapes and speech discrimination materials. Aspects of test development and test results will be discussed in detail. [This research was supported by contract with the U. S. Bureau of Mines.]

EE5. Replication of Frequency Response Aspects of Hearing Loss for Use in Communication Studies. JAMEs H. Prout AND Gordon Bienvenue, Environmental Acoustics Laboratory, The Pennsylvania State University, University Park, Pennsylvania 16802.- - In studying the problems of communication among coal miners, it was desirable to replicate electronically certain aspects of their hearing abilities so that one group of normal-hearing subjects could be used for the entire study. Tests were designed to determine the ability of miners to hear speech and nonspeech signals in noise and quiet, with and without ear protectors. Since miners cover a wide range of ages with varying amounts of hearing loss, the literature dealing with hearing of noise-exposed miners was surveyed and collated to obtain audiograms of three representative subgroups. From this threshold data, a linear transformation was used to obtain equal loudness contours for bands of noise. Three electrical filters were then developed from this data to approximate the frequency response aspects of the miners' hearing loss. The derivation of the filters and some results of tests involving their use will be described. [This research was supported by a contract with the U. S. Bureau of Mines.]

EE6. A Computerized Audiometric System for the Fail-Detect Monitoring and Self-Calibration of Frequency and Hearing Level. T. J. Wood, Institute of Speech and Hearing Sciences, University of North Carolina, Chapel Hill, North Carolina 27514. -Past research has indicated the need of frequent monitoring and calibration of audiometric stimuli. This study investigated 
the applicability of an automatic monitor and self-calibration system for frequency and hearing level employing relevant computer peripheral equipment and a mini-digital computer. Unacceptable drifts in frequency and hearing level were intentionally introduced into the system by respectively turning the frequency selector dial of a function generator and an attenuator. After monitoring and, if indicated, computerized calibration, the signals were subsequently sampled via traditional calibration equipment to determine the frequency and hearing level parameters of the computer-revised signals. The obtained data were analyzed in terms of means, standard deviations, average deviations, and maximum errors encountered at each of six audiometric frequencies $(250 \mathrm{~Hz}-8 \mathrm{kHz})$. The results of this study indicate the feasibility of providing frequent stimulus monitoring and self-calibration within a system of computerized audiometrics. [This research was supported, in part, by the University Research Council of the University of North Carolina.]

EE7. Acoustical Signature of Some Hand Guns Commonly Employed in Law Enforcement. M. T. Kobal and P. G. WEISSLER, Institute for Basic Standards, National Bureau of Standards, Washington, D. C. 20234.-Small condenser microphones and a storage oscilloscope were used to record the $N$-wave signatures from a 9 -mm Ruger, 0.357 magnum, 0.41 magnum, 0.22 long rifle, 0.44 magnum, 12-gauge shotgun, and 0.22 rifle. Measurements were made at the ear of the person shooting and at the approximate position of a neighbor at a firing range. Data on peak SPL and durations $(A$ and $B$ durations) are given, as well as data obtained at a practice firing range. The results are discussed from the point of view of using ear defenders to protect against temporary and permanent threshold shift incurred during practice. [Research supported by the National Institute of Law Enforcement and Criminal Justice.]

EE8. Pure-Tone and Speech-Evoked Responses in Normal and Noise-Exposed Squirrel Monkeys. Richard P. MaIoriello and Chester R. Wilpizeski, Department of Otolaryngology, Thomas Jefferson University, Philadelphia, Pennsylvania 19107.-Monkeys were implanted with permanent recording electrodes having tips placed in the auditory nerve-cochlear nucleus region. AP input-output relationships and VDLs were obtained from computer-averaged evoked responses to pure-tone pulses and selected monosyllabic words before and after five consecutive daily $15-\mathrm{min}$ exposures to $115 \mathrm{~dB}$ SPL of steady broad-band noise. Threshold shifts and sustained reduction in AP amplitudes occurred at higher frequencies. Response patterns evoked by speech appeared coded in the form of amplitude modulation, and words could be identified by the summed waveform associated with each stimulus. Response patterns for some words were altered by noise exposure. Results suggest that noise intensity-duration combinations currently accepted as nonhazardous to hearing (on the basis of TTS recovery) may produce persistent changes in hearing function for suprathreshold sounds, and that speech reception may be altered by noise stimulation followed by only minımal changes in pure-tone VDLs.

EE9. Asymptotic TTS in Chinchillas. William Melnick, Mariann Migliore, and David Lim, Department of Otolaryngology, Ohio State University, Columbus, Ohio 43210.Six chinchillas were monauralized and were trained to produce behavioral audiograms using a shock avoidance procedure. The animals were then exposed to a 300 - to $600-\mathrm{Hz}$ octave band of noise at $85 \mathrm{~dB}$ octave band level for a period of $96 \mathrm{~h}$. Thresholds were measured at 10 test frequencies ranging from 250 to $8000 \mathrm{~Hz}$ at varying intervals during the exposure. Results from the present investigation were remarkably similar to those reported by Carder and Miller (1960). The growth of TTS followed a triphasic pattern. Initially there was a period of slow growth which lasted approximately 6-12 $h$; then there was a period of rapid growth, and finally an asymptote was reached after a period of $24-48 \mathrm{~h}$. The asymptote obtained in the present investigation was predicted fairly well by the equation TTS $=1155$ (octave band level -65 ). All 10 of the test frequencies showed TTS. Recovery from asymptotic TTS was complete for nost animals by 12 days postexposure, slightly longer than that reported by Carder and Miller. [Partially supported by Air Force Contract.]

EE10. Behavioral and Anatomic Manifestations of Acoustical Overstimulation. C. Trahiotis, H. W. Ades, A. KokkoCunningham, and A. Averbuch, Department of Psychology and Department of Electrical Engineering, University of Illinois, Urbana, Illinois 61801 . - The data to be discussed represent preliminary attempts to investigate further the relations between acoustic overstimulation, cochlear pathology, and behavioral indices of auditory discriminability. Monaural chinchillas served as subjects and were tested by traditional avoidance techniques to detect pure tones in "quiet" and in various levels of broad-band noise. Stimuli producing acoustic overstimulation included various levels of $4-\mathrm{kHz}$ pure tones and bandpass noise whose greatest spectral levels occurred between 1 and $2 \mathrm{kHz}$. Both "quiet" and masked postexposure thresholds will be compared to the cochleograms obtained six months following stimulation. [Supported by grant from NASA.]

EE11. Audiometric and Histological Effects of Exposure to 40- $\mu$ sec High Level Impulses. Donald Henderson, Roger P. Hamernik, and Ronald W. Sitler, Department of Otorhinolaryngology, State University of New York, Upstate Medical Center, Syracuse, New York 13210.-Monaural chinchillas (15) were exposed to 50 impulses of either 158,615 , or $175 \mathrm{~dB}$ peak SPL. The impulses were generated by a spark-gap source, which produced an impulse with a 40- $\mu$ sec $A$ duration. Quiet thresholds were measured at $0.25,0.5,1,2,4$, and $8 \mathrm{kHz}$, using the averaged evoked response technique. Recovery was monitored for 31 days and then the animals were sacrificed and their cochleas were analyzed using the surface preparation technique. The three exposures produced virtually no permanent threshold shifts (PTS) with the exception of 10-30 dB PTS in one animal in each of the 165- and 175-dB groups. However, hair cell losses were seen in all three groups. The cochleagrams varied from low level scattered losses to lesions of the OHC that spanned a 4-mm stretch in the base of the cochlea. The IHC losses were considerably smaller and more restricted. The audiograms do no necessarily reflect the state of the cochlea.

EE12. The Interaction of Continuous and Impulse Noise: Audiometric Effects. James J. Crosslev, Richard J. Salvi, Roger P. Hamernick, and Donald Henderson, Department of Ororhinolaryngology, State University of New York, Upstate Medical Center, Syracuse, New York 13210.-Continuous and impulse noises, considered "safe" by existing damage risk criteria (DRC), were combined to model more realistic noise environments. Monaural chinchillas (23) were exposed to one of the following conditions: (a) $95 \mathrm{~dB}$ SPL continuous noise at $2-4 \mathrm{kHz}$ for $1 \mathrm{~h}$; (b) 50 impulses with a $40-\mu \mathrm{sec} A$ duration at $158 \mathrm{~dB}$ SPL peak pressure; (c) interrupted combination of the continuous and impulse noise with the continuous noise off for 2 sec during the impulse delivery; (d) superimposed combination of the continuous and impulse noise. Quiet thresholds were measured before and after exposure at $\mathbf{0 . 2 5}$, $0.5,1,2,4$, and $8 \mathrm{kHz}$ using the averaged evoked response technique. Recovery was monitored for 31 days. The median recovery from the $95 \mathrm{~dB}$ SPL continuous and $158 \mathrm{~dB}$ impulse was complete by $30 \mathrm{~h}$. The interrupted combination produced 
temporary threshold shifts (TTS) of 50-60 dB at 2, 4, and 8 $\mathrm{kHz}$ that required four days to recover. The superimposed combination produced, $30 \mathrm{~dB}$ TTS at $0.25,0.5$, and $1 \mathrm{kHz}$, 70-90 dB TTS at 2, 4, and $8 \mathrm{kHz}$, and a final median audiogram typical of noise induced sensorineural hearing loss with $30-40 \mathrm{~dB}$ PTS at 2,4 , and $8 \mathrm{kHz}$.

EE13. The Interaction of Continuous and Impulse Noise: Histological Effects. Roger P. Hamernik, James J. Crossley, Donald Henderson, and Richard J. Salvi, Department of Otorhinolaryngology, Slate Universily of New York, Upstate Medical Center, Syracuse, New York 13210.-The cochleas of the chinchillas exposed to the four noise conditions described in the previous abstract were examined using the surface preparation technique. The animals (six) exposed to the continuous noise alone, had essentially normal hair cell populations. The animals (five) exposed to the 158- $\mathrm{dB}$ peak impulse had hair cell losses that ranged from a scattered low level loss toapproximately a $4 \mathrm{~mm}$ loss of OHC in the base of the cochlea. IHC losses were considerably smaller. The animals (six) exposed to the interrupted combination had nearly the same range of hair cell losses as seen in the 158-dB group, with the exception of one animal that had a large $(8 \mathrm{~mm})$ discrete loss of $\mathrm{OHC}$ and IHC. The superimposed combination produced the most severe hair cell lesions with five of the six animals showing nearly $100 \%$ lossses of $\mathrm{OHC}$ and IHC extending throughout the basal one-third to one-half of the cochlea. The audiometric and histological findings agree in showing that the superimposed combination of the two "safe" noises produces traumatic effects that more than exceed the additive effect of either component. The existing DRC do not provide guidlines for such combinations.

EE14. An Evaluation and Description of the Electroacoustic Units Associated with the Verbo-Tonal System. C. W. Asp, J. E. Keller, AND R. P. Williams, Department of Audiology and Speech Palhology, Univesity of Tennessee, Knoxville, Tennessee 37916. -The verbo-tonal method advocates working through the impaired auditory modality as the primary channel to habilitate or rehabilitate hearing-impaired people. To implement this philosophy, six electroacoustic units have been designed. The characteristics of these units are as follows: (1) Suvag I has an electrical response that extends from $\frac{1}{2}$ to $20000 \mathrm{~Hz}$; it can also be utilized in low-pass filter conditions of $0.6,1,2$, and $3 \mathrm{kHz}$. (2) Suvag II is a multichannel unit with the following modes of operation: (a) direct channel (20-
$20000 \mathrm{~Hz}$ ); (b) low-pass and also low-peaking filters of 0.07 , $0.15,0.3,0.6$, and $2 \mathrm{kHz}$; (c) high-pass and also high-peaking filters of $3,4,6$, and $8 \mathrm{kHz}$; and (d) base and treble control of + and $-16 \mathrm{~dB}$. (3) Suvag Lingua has discrete bandpass filters that correspond to the "optimal octaves" of normal-hearing people; it is utilized for teaching foreign languages. (4) The Mini Suvag hearing aid is a body-type aid with a wide frequency response. If utilized with a separate power amplifier, the unit can simulta neously drive both bone- and air-conduction transducers. (5) The audiometer is utilized to present prerecorded filtered speech signals. (6) The Suvag Vibar is a bone vibrator that is placed at different points of the body during the therapy procedure. Electrical and acoustic frequency responses will be displayed and compared to those of other units. [Work supported by the U. S. Office of Education.]

EE15. Evaluation of Low-Frequency Amplification, Time in Therapy, and Testing Conditions for Preschool Deaf Children. C. W. Asp, E. H. French, J. S. Berry, AND J. E. Keller, Department of Audiology and Speech Pathology, University of Tennessee, Knoxville, Tennessee 39716.-Fifty-five deaf children were tested at four-month intervals with 27 test words. The factors under test included: (1) type of amplification (wide band versus narrow band); (2) testing times; (3) auditory versus visual and auditory clues; and (4) testing with amplification versus testing without amplification. Records were kept on the familiarity of the test words and the number of responses. Length of enrollment in the program was based on the availability of each child over the five-year period of this study. The verbo-tonal method was utilized to habilitate all the children. For the third, fourth, and fifth years, an experimental design [C. W. Asp, J. Acoust. Soc. Amer. 48, 87 (A) (1970)] permitted the teacher to speak simultaneously through two different amplifying systems, a wide-band auditory training unit (Suvag I) and a narrow-band unit (Warren T-2). For one criterion measure, 20656 speech samples were recorded, randomized, and judged on a 9-point scale. Preliminary statistical analyses suggested the following: (1) in general, children assigned to the wide-band unit improved at a faster rate than those assigned to narrow-band units; (2) significant improvements were detected over the testing times, (3) the scores were better when both visual and auditory clues were available and also when amplification was available. Data for additional criterion measures are being processed for future reports. [Work supported by U. S. Office of Education. Acknowledgment is offered for the many staff members associated with this project.]

Thursday, 30 November 1972

Dominion RoOM, 2:00 P.M.

\author{
Session FF. Shock and Vibration IV : Analytical and Experimental Studies \\ of Vibrating Systems
}

Charles T. Molloy, Chairman

Systems Group of TRW Inc., Washington Operations, McClean, Virginia 22101

Contributed Papers (10 minutes)

FF1. Abstract withdrawn.

The Journal of the Acoustical Society of America 
FF2. Resonant Stresses in Structures Based upon Experimental Response Data. L. D. Mitchell and M. C. Tod, Engineering Materials Laboratory, E. I. du Pont de Nemours \& Co., Wilmington, Delaware 19898.-A combined experimental and analytical approach to the problem of resonant stresses is presented. The approach is formulated so that the mechanical dynamic eigenvalue-eigenvector analysis can be used with a single-point mechanical mobility measurement on the structure to predict the resonant stresses at any point in the structure.

FF3. Transient Response of Multi-Degree-of-Freedom Linear Systems to Forcing Functions with Inequality Constraints. C. D. Michalopoulos, Department of Mechanical Engineering, University of Houston, Houston, Texas 77004.-Optimal control theory is employed to analyze the transient response of discrete linear systems to forcing functions whose dependence on time is not known, but which lie within specified bounds. Attention is focused on the complete determination of the forcing function that will induce (a) maximum displacement of any specified mass element; (b) maximum relative displacement of any two adjacent masses; and (c) maximum acceleration of a given mass. Undanıed mechanical systems with an arbitrary number of degrees of freedon are considered wherein only a single forcing function acts. In general, the desired forcing function is found to be a bang-bang type function, that is, a function which switches from the upper to the lower bound and vice versa at certain instants of time. The general procedure for finding these switching times is presented.

FF4. Nonlinear Transverse Vibrations of Inhomogeneous Beams. Ali H. NAYFEH, Engineering Mechanics Department, Virginia Polytechnic Institute and State University, Blacksburg, Virginia 24061.- The method of multiple scales is used to analyze the nonlinear transverse vibrations of inhomogeneous beams including the effects of transverse shear and rotary inertia. First-order uniform perturbation expansions are derived for the displacements, shear force, and frequency in terms of the ratios of maximum transverse displacement to cross-sectional radius of gyration and cross-sectional radius of gyration to wavelength.

FF5. Approximate Fundamental Frequency of Noncircular Ring Membranes. F. E. EASTEP, Department of Mechanics, Air Force Institute of Technology, Wright-Patterson Air Force Base, Ohio 45433.-An exact solution for the frequency of a ring membrane can be obtained when the inner and outer boundaries are concentric circles or confocal ellipses. If either boundary has some other shape, approximate methods must be used. This paper presents a simplified approximate method for determining the fundamental frequency of ring membranes whose boundaries deviate only slightly from circular. This method was initially used by Rayleigh in his investigation of simply connected membranes. The radii of both bounding curves are approximated by a truncated Fourier series. The membrane transverse motion, which is written as a superposition of the modes of a circular ring membrane, is forced to vanish on the approximated boundaries. A characteristic equation is obtained from which the approximate frequencies may be found. This technique is used to determine the fundamental frequency of the following: (1) Elliptical ring membrane with the inner and outer ellipses being confocal; (2) square membrane with a centered circular hole; (3) square ring membrane; and (4) an eccentric annulus. Comparisons with exact solutions, when known, are given.

FF6. Free Oscillation of Thin-Walled Open-Section Circular Rings: A Conventional Approach. N. R. MADdox, Esso Production Research Center, Houston, Texas 77001, AND G. M. Rentzep1s, School of Engineering Science and Mechanics, Georgia Institute of Technology, Atlanta, Georgia 30332.-A theoretical study of the eigenfrequencies for a complete thinwalled open-section circular ring with one plane of symmetry in the plane of the ring is conducted. The analysis consists of a conventional theory incorporating St. Venant torsion, Timoskenko warping, torsional inertia, and, for the first time, the shear center eccentricity from the center of gravity for out-ofplane free oscillations and extensionality for in-plane free oscillations. This approach, including all the effects mentioned above, yield four coupled equations of motion for the free oscillations. With the plane of symmetry lying in the plane of the ring, this system of equations splits into two coupled sets; one for the in-plane oscillations, the other for the out-of-plane oscillations. The complete ring eigenfrequencies correspond to eigenmodes of four or more nodes resulting from the values available in the literature even for slender rings; this is attributed to the incorporation of the shear center eccentricity-an effect that has been previously ignored. The out-of-plane ring equations have been found to reduce to ring equations of motion available in the literature by elimination of various parameters. Studies of these various systems of equations of motion have shown that the effect of the shear center eccentricity on the natural frequencies of rings with radii ratios as low as $1: 50$ is not negligible. Hence, a vibration study involving monosymmetric rings should include the eccentricity of the shear center if a reasonable prediction of natural frequencies and/or the response of a system to a given forcing function is being sought. If, in addition, one neglects the warping, the results are completely erroneous for thin-walled rings.

FF7. Response of a Helical Coil to a Step Impact Load. D. W. Haines and N. Chang, College of Engineering, University of South Corolina, Columbia, South Carolina 29208. -A theoretical solution is presented of a one-turn elastic helical coil of small pitch with one end fixed and subjected to a step impact load applied tangentially to the other end. Previously derived onedimensional equations [Paper H8, 83rd Meeting of the Acoust. Soc. Amer.] are taken as the governing equations of motion. 
The Mindlin-Goodman method is applied to solve these equations with the boundary conditions as indicated. The solution demonstrates the effertiveness of the coil as an impact absorber. [This work was supported by the National Science Foundation.]

FF8. Vibration and Sound Radiation of a Plate. R. J. HANNon, Physics Depariment, The Pennsylvania State University, University Park, Pennsylvania 16802.-Because of resonances, plates are acoustically soft. The experimental evaluation of the acoustic properties of plates is difficult since they are usually supported at points which act to clamp the plate. To eliminate this, a plate was placed on a cushion of open-cell plastic foam, and the velocity amplitude due to a point force was determined with a record player cartridge and a microphone in the near sound field. The results show that the many modes that are excited interact to generate "hot spots" on the plate. The attachment of ribs and supports disturbs the vibration field and leads to localized area of sound radiation.

FF9. Abstract withdrawn.

FF 10. Exact Solution for Transmission Matrix of LiquidFilled Pipe Using Sylvester's Theorem. D. R. SAMSURY, Naval Ship Research and Development Center, Annapolis, Maryland 21402. - The differential equations describing the vibrational behavior of a liquid-filled curved pipe are presented. The corresponding eigenvalue problem is formulated in terms of the coefficient matrix of these equations in the usual $(\lambda I-B)$ form. With the aid of a special computer program, general polynominal expressions for the elements of the adjoint of $(\lambda I-B)$ and its characteristic equation are obtained in terms of $\lambda$ and elements of $(B)$. A simple matrix operation is used to validate their form. Solution of the characteristic equation yields the eigenvalues and thus values for the adjoint matrix. Using this information and Sylvester's theorem, the desired transmission matrix $(U)$ is obtained. $(U)$ reflects the effects of shear deformation, rotary inertia, damping, and cross-sectional deformation. Difficulty in obtaining the $\mathbf{1 4}$ distinct eigenvalues is discussed as is the case of repeated eigenvalues. The final transmission matrix is compared to one obtained by a demonstrably accurate numerical approximation method.

FF11. Prediction and Measurement of the Proportionality Constant in Statistical Energy Analysis of Structures. ROBERT Lotz, U. S. Department of Transporlation, Transportation Systems Center, Cambridge, Massachusetts 02142, AND STEPHEN H. Crandall, Massachusetts Institute of Technology, Cam- bridge, Massachusetts 02139.- The fundamental equation of sta tistical energy analysis (SEA) states that the average power flow between two coupled vibrating systems is proportional to the difference in their average modal energies. Under certain circumstances, one can estimate the proportionality constant by modifying system boundary conditions on the separated systems and calculating or measuring changes in the systems. Newland's estimate, based upon blocking part of the system, is reexamined and limitations are discussed. Three alternative methods which circumvent blocking are presented. They are based, respectively, upon (1) natural frequency shift due to addition of mass; (2) mechanical input impedance; and (3) mean square velocity response at the excitation point due to random excitation. The new methods were applied to predict power flow in experiments on coupled beams and on coupled plates. One or both parts of the coupled structures were then excited by external random sources, and power flow through the coupling was measured directly as a product of force with velocity. The fundamental SEA relation was supported by the measurements, which included the null power point where the difference in average modal energies was zero. Measured and predicted proportionality constants are compared. [This work was supported in part by the National Aeronautics and Space Administration.]

FF 12. On Modeling the Vibration Characteristics of a Chain Saw. D. D. Reynolds, Department of Architectural Engineering, University of Texas, A ustin, Texas 78712, AND W. SOEDEL, Department of Mechanical Engineering, Purdue University. Lafayette, Indiana 47906.-The vibration response characteristics of a chain saw were modeled, treating the saw as a rigid body supported by a system of springs and dampers at the two handle locations of the saw. The arrangement and values of the coefficients for the springs and dampers were experimentally selected to result in the same dynamic response as if human hands were clasping the chain saw handles. The saw was modeled as a $7 \mathrm{df}$ (degree of freedom) system-three translational directions, three rotational directions, and one to describe the rotational motion of the slider-crank mechanism of the I.C. engine of the saw. The kinematic and dynamic properties of the slider-crank mechanism were exactly modeled. The analytical results of the model for the fundamental forcing frequency agreed very well with the experimentally measured results, of ten within $10 \%$ of each other. Because of structural resonances of the saw body, which were not incorporated into the model, the agreement between the analytical and experimental results at the harmonic frequencies of the fundamental forcing frequencies was not as good.

FF13. Development of a Finite Element Model for a Guided Spherical Shell. G. Georgopoulos and L. H. Royster, Center for Acoustical Studies, Department of Mechanical Engineering, North Carolina Slate Lniversity, Raleigh, North Carolina 27606. -A doubly curved anisymmetric spherical-shell finite element is utilized in order to predict the dynamic response of spherical shells for the Class V flextensional underwater acoustic transducer. The spherical shell is represented as a finite number of spherical frustra, joined at nodal circles. Norozhilov's "Thin Shell Theory" is applied and the element stiffness and mass matrices are derived and numerically integrated by utilizing Gauss-Legendre polynomials. The frequency coefficients of a spherical shell are defined. An IBM 360/75 computer is used for obtaining numerical results for varying thickness and opening angle, with guided clamped, guided pinned, clamped, and pinned boundary conditions. Numerical results for the Class $V$ flextensional underwater transducer shell are included. Finally, a convergent test is carried out for a different number of finite elements for the Class $\mathrm{V}$ flextensional underwater transducer shell having pinned boundary conditions. The results agreed almost perfectly with earlier results obtained experimentally. 


\title{
Session GG. Underwater Acoustics V : Scattering; Noise
}

\author{
BLRTL: (;. IILRLE, Chairman \\ U. S. Nazal Research :aboratory, Washington, D. C. 20390
}

\section{Contributed Papers (10 minutes)}

GG1. Abstract withdrawn.

GG2. Underwater Modulation Effects. H. S. HAYre, Professor and Director of II'ave Propagation Labs, Electrical Enginier'ig Department, University of Houston, Hmiston, Texas 77001.The use of Kirchholf-Huygens' integral is made to show that both amplitude an! angle modulation are produced by the relative motion of tie signal source and the randomly scattering ucein surface or the target with its , isociated random modium elfects. Furthermore it is shown that these two modulation effects are statistically dependent. The case of a Doprier system where the ant $: 1$ itude modulation efferts are essenticl $1 ! y$. eliminated by hard liniting is studied. Tlit refore the dependence of the Doppler signal spectrum and is bandwidth on the - ifare roughness, as well as the angle of incidence, are derivr.d tor an operational frequency of $50 \mathrm{kHz}$. The rates of one-dimensional isotropic surfaces, as well as surfaces witi along- and cross-wind, statistically independent, spatial height correlation functions are considered. The results are expressed in empirical form $:$ to show their 1 -efulness to syster. designers and operational personnel.

GG3. Doppler Spreading of Reverberation in Shallow Water. J. Ml. Ross AND C. BRor:HU, Defence Research Establishment. Allantic, Dartmouth, Nova Scotia, Canada.-Reverberation data have been gathered with a narrow-beam transducir, which 1 r.1nsmitted a $1 . \therefore$ sec $\mathrm{CW}^{\prime}$ pulse at $9.5 \mathrm{kHz}$. The tra.,-ducer was towed thro.. : l'ne waters of the Scotian Shelf and Gulf of St. Lawrence at various depths. Conditions exist in these relatively shallow water areas where surface, volume, and boilom are all, at different times, the dominant h.ickscatteri:rg mechanism. In winter, because of the tylik aily strong positive thermal gradients, surface reverberation dominates. In summer, two common situations exist: $i_{i}$. strong negative gradient, where botton is the domiluant backscatterer, and (2) a pronounced sound channel whertvolume may be the dominant backscatterer if leakage is - mall. If sufficient leakage occurs, contributions from bottom and volume reverberation can both be significant. When surface reverberation dominates, spectral bandwidtlis are a function of the sea surface conditions. When bottom reverberation is dominant, the spectral spreading is greater than that associated with the system resolution, probably due to variations in shear currents. The spreading associated with volume reverberation lies between that of bottom and surface backscattering, but the scattering strength is significantly lower.

GG4. An Acoustic System for Measuring the Waveheight of Sea Surface Waves. WILliam I. Roderick,* New Landon Laboratory, Vaval Underwater Systems Center, New London, Connecticut 06320.-An upward-looking echo-sounder system has been designed to measure the time varying waveheight of sea-surface waves. A narrow beam of pulsed acoustic energy was focused on the sea surface by utilizing the transitional region from the near to farfield characteristics of a circular piston transducer. The transducer, operating at $360 \mathrm{kHz}$, was pulsed at $10-$ msec intervals to sample the waveheight. $B_{j}$ transmitting short pulse lengths, the wide-band characteristics of the transducer were used to discriminate against nearsurface bubble reverberation. The system is linear and can presently measure surface wave frequencies below $4.5 \mathrm{kHz}$. Waveheights as small as 0.05 in. were measured in a model tank by comparing the echo-sounder results with results obtained from a micrometer. An experiment, conducted in the Thames River, compared the performance of the echo sounder to a $15-\mathrm{ft}$ resistive wave staff. Comparisons of ensembleaveraged power spectral densities obtained from the wave staff and the transducer, positioned $10 \mathrm{ft}$ below the surface, were excellent for wave frequencies below $4.0 \mathrm{~Hz}$.

* Currently a nonresident research student with the University of Birmingham, Birmingham 15 , England.

GG5. Deep-Scattering-Layer Observations off New Zealand. W. E. Batzler, Naval Undersea Center, San Diego, California 92132.-Acoustic volume scattering measurements made during the 1971 winter season at 18 deep-water stations north and east of Auckland. New Zealand, are presented in terms of volume scattering strength and column strength. (Columns extend from near surface to a depth of $1000 \mathrm{yds}$.) Both pulsetype data, at 5.0 and $12 \mathrm{kHz}$, and explosive-source data were obtained. At a given frequency, there is little station-tostation change in column strength over the $\frac{1}{3}$-oct bands from 1 to $20 \mathrm{kHz}$. Typically, column strength rises sharply from 1 $\mathrm{kHz}$ to a maximum at 5 or $6 \mathrm{kHz}$ followed by a slight droop and another maximum at $16 \mathrm{kHz}$. Values fall in the range -40 to $-70 \mathrm{~dB}$. With one exccption, night column strengtlis are higher than day values; this day-to-night difference has a maximum in the neighborhood of $3 \mathrm{kHz}$. The pulse results, expressed as volume scattering strength versus deptli, also show station-to-station similarities; the principal scattering layers occur at about the same depths, and column strengths do not differ greatly. The distribution of volume scattering strengths with depth reveals distinguishing station-to-stalion differences not provided by the explosive data. Column strengths obtained from pulse data are in satisfactory agreement with those obtained from explosive measurements. Comparisons show that scattering levels off New Zealand are as high or higher than those observed in other North and South Pacific areas. 
GG6. Measured Effects of Ice Roughness on undar-Ice Transmission Loss. O. I. Diachok and T. L. Kozo, $C$. $S$ Naval Oceanographic Office, IVashington, D. C. 2039\%.-Coincident measurements of under-ice transmission loss over a frequency range of $100-1000 \mathrm{~Hz}_{2}$ and top ice roughness were made from aircraft. The acoustic measurements were made with calibrated sonobuoys and explosive charges, while top ice roughness measurements were made using a laser profiler along several $100-\mathrm{km}$ tracks. The resulting ice roughness data is expressed in terms of ridge density. Is expected, lowfrequency transmission loss increases with top ice ridge density. According to a preliminary analysis of top and bottom ice roughness data, top ice ridge density is a quantitative indic at or of bottonside ridge density and rms roughness. The lata :.te compared with theoretical predictions and previous mcasurements. Some possible acoustic scattering mechanism at the water-ice interface are suggested.

GG7. Under-Ice Reverberation Modeling by Means of Multiple Regression. W. B. BIRCH, Newporl Laburatory, Naval Undemwaler Systems Center, Newport, Rhode Island 02840.-Multiple regression techniques are being used to produce a model of under-ice reverberation when minimal information exists concerning the nature of the under side of the ice. Field recorded acoustic data have been used to generate a series of equations that estimate the reverberation signal at the transducer face for each of several times following transmission of an acoustic pulse. The highest percentages of explained deviation have been obtained using a linear regression equation of the form

$$
Y^{\prime}=b_{0}+b_{1} X_{1}+b_{2} X_{2}+b_{3} X_{3}+b_{4} Y_{4}
$$

where $Y^{\prime}$ is the signal amplitude; $X_{1}, X_{2}, X_{3}, X_{4}$ represent, respectively, the independent variables of transducer elevation angle, pulselength, depth, and beamwidth; and $b_{0}, b_{1}, b_{2}, b_{3}, b_{4}$ are the regression coefficients. Confidence limits for the coefficients and the relative contribution of eacl variable to the regression are provided by an analysis of variance. The final model is intended to permit prediction of the reverberation sound pressure level at a transducer face for known transducer acoustic and physical parameters.

GG8. A Simplified Description of Wind-Generated Noise for Sonar Response Applications. G. G. Hugrolns and C. B. Burroughs, Bolt Beranek and Newman Inc., Arlington, Virgina 22209.-Many current ambient tusise measurement and modeling programs are aimed at imporved estimates of the spatial properties of ambient noise fields. One application of these studies is the development of models for predicting sonar array response to anisotropic ambient noise. The purpose of this paper is to present a simple model developed in closed form for sonar applications, and yet which reflects the dependence on important environmental parameters. The model describes vertical directionality of wind-generated noise above and below the horizontal for a near-surface receiver. The sensitivity of the model to such factors as the strength and sign of the velocity gradient, bottom loss, and water depth are explored. Comparisons to more accur : te and comprehensive models and measurements show that tin model satisfactorily describes the substance $"$ ', these environnental factors. For example, noise directionality above the horizontal for a shallow receiver is only weakly dependent upon the velocity gradient. Below the horizontal, results are strongly a function of bottom reflectivity and nearly independent of water depth. [Study supported by NAVSHIPS, PIiS 302-4.]

GG9. Sonar Array Response to Ambient Noise Directional Fields, Comparing Numerical Results to Closed-Form Estimates. G. G. Huggins and C. B. Burroughs, Boll Beranek and Newman Inc., Arlington, Virginia 22209.Several studies have been directed towards the development of both numeric

and 1 ical expressions of ambient

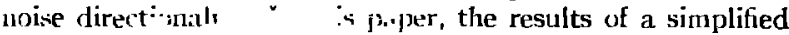
ambient noise model are implo rer to examine the response of

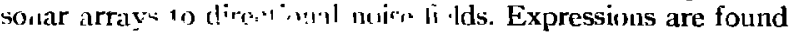
for the array gain ag. $117 \mathrm{t}$ wind noise for near-surface arrays, employing vertical and horizontal line arrays is examples.

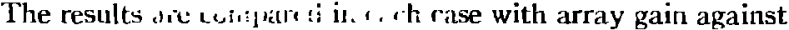
isotropic noise (i $e, y, \ldots: y$ iadex). Array response is found as a function of . rr-1: rientation, freque cy (or size), rnd steering angle. For tl.. loroadside steered ine array, a simplified beam responst pattern is used to find array gain for these simple allaly. - is models. Results compare very well with a1: $:$ mputed using more precise models of il. it. $\quad \therefore$ and exact a. ray response patterns. The $1.1_{1}$.ucati.. . - onar design ani jerformance studies are discmicied, : - : , similar estima. for the response of more complex sriay configurations. LStudy sup-

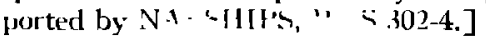

GG10. Ambient Iinas ...utration at the Arctic Ice-Water

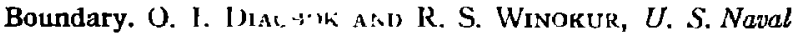
Oceanographic Office, !iashinglom, D. C. 20390.-Quasisynoptic measurements were made to determitte the spatial variability of ambient noise at the Arctic ice-water boundary over a frequency range from 1110 to $1000 \mathrm{~Hz}$. The results of this investigation show that the ice-water boundary acts as a spatially well-delined sourcr af ambient noise with measured

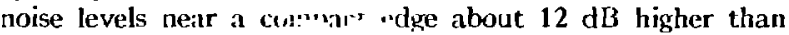
open water levels and ,imul, (1) $\mathrm{dB}$ higher than levels far in the ice field. Measured noise levels near a diffuse ice edge were about $4 \mathrm{~dB}$ higher than open water levels, and about 10 $\mathrm{dB}$ higher than levels far in the ice field. The relatively high noise levels are probably generatid by wind-wave interactions with ice floes. Specific mechinlisms of noise grneration are suggested, and the measured data are compared to ambient noise data in the rentral Arctir.

GG11. Long-Term Ambient :1،:ise Measurements in the N.E. Pacific Ocean. J. A. Scrin. “', Defence Research Establishment Pacific, F.M.O., Vict. i, B.C., Canada.-Long-term (one-year) open ocean mea surements of ambient acoustic noise were made in the N.E. l'acific Ocean using instrument packages placed on seamounts. The seamounts $\mathrm{Cobb}$ [summit 15 fathoms (f)], Union ( $: 11 \%$ ' $\mathrm{ii} 160 \mathrm{f}$ ), and $\mathrm{B}$ ' wie (summit $16 \mathrm{f}$ ), extended over $6 \frac{1}{2}^{\circ}$, iatitude in a near $\$-\$$ direction. Packages were placed oi salmunnts Cobb and Bowie on terraces at about the 110-f level and on Union near the summit at about the 170-f level. Averaged 4-min samples of noise level in the $150-$ to $317^{\circ}-\mathrm{Hz}_{2}$ frequency band were obtained once per hour and recorded as a bar graph on strip paper chart. Usable data $y^{\left.\mathrm{i}_{\mathrm{l}}\right]^{3}}$ was about $50 \%$. The data are presented in the form of histugrams of frequency of occurrence of noise level in 5- $\mathrm{dB}$ intervals by months. There appears to be about a 4-dB increase in noise level in the band i. the winter months. A hrief description of the instrument parkage and the technique used for placement and recovery will be given.

GG12. The Effect of Ice Cover on Shallow Water Ambient Noise. A. J. Collick, Deferce Research Establishment Atlantic, Dartmouth, Nova Scotia.--Ambient sea noise in the frequency range 12.5-6100 $\mathrm{H}_{z}$ was measured in the Cabot Strait, near Cape North. Nova Scotia, during the period 23 February to 16 April 19;1 . b bicom- tounted hydrophone at a $350 \mathrm{ft}$ depth. During this period, the loe ice moved continuously,

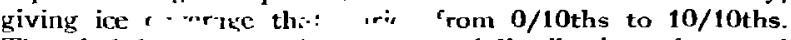
The wind-deph...dent noise : : sclial and distributions of spectral levels are presented for various degrees of ice coverage. It was found that the spectral levels are wind dependent for all degrees of ice cover, $i \cdot i$ the under-ice spect $\cdot$ ll levels are 


\title{
84TH MEETING - ACOUSTICAL SOCIETY OF AMERICA
}

significantly lower than the open water levels for comparable wind velocities.

GG13. Acoustic Assessment of North Pacific Salmon Stocks. W. C. ACKer, Applied Physics Laboralory, University of Washington, Seattle, Washington 98195.-Acoustic sampling of various fish stocks and the acoustic characteristics of fish targets are being investigated as part of the Marine Acoustics Program at the University of Washington. An important outgrowth of this research is the development of a free-floating acoustic system which is being used to help index the North Pacific salmon population. This is an uplooking system which operates at $250 \mathrm{kHz}$. Average target size of the migrating salmon is about - $30 \mathrm{~dB}$ within the aspect angles encountered in the sample volume. Acoustic source level and TVG receiver sensitivity were adjusted for that average size, but a fairly wide range of target-size fluctuation can be accommodated $( \pm 10-15 \mathrm{~dB})$. Studies of relative abundance indicate that the population density in particular areas is related to the spawning migration to Bristol Bay, Alaska, the following year thus providing an index of population abundance [D. E. Rogers, Fish Rec. Inst. Circ. \# 70-7 (1970)]. A second acoustic system is now being developed for use in Bristol Bay to generate population data upon which to base the day to day management of the fishery so that catch and escapement are optimized.

Thursday, 30 November 1972

Silver Chimes East, 2:00 P.M.

\section{Session HH. Speech Communication VI : Speaker Identification, Speech Recognition, and Synthesis}

\author{
A. Michael Noll, Chairman
}

EOP/OST, Washington, D. C.

This is a special session; preprints provided; discussion only.

\section{Contributed Papers}

HH1. Effects of Context on Voiceprint Identification. BARRY HAzEN, University of North Carolina, Chapel Hill, North Caralina 27514.- This study investigated how well the file card system of Voiceprint Identification reported by Kersta (1962) fulfils its purpose of minimizing the effects of contextually caused spectral variation, and how well it serves as either a population reduction or identification tool. Subjects received training equivalent to that received by the experimenter in the Voiceprint Identification Training Course, and achieved error rates approximating those achieved by Kersta's subjects for similar training tasks. Experimental tasks required subjects to identify unknown speakers from a population of $\mathbf{5 0}$ known speakers by first eliminating all known speakers they were certain were not the unknown speaker, and then attempting identification. Ten attempts were made where the unknown and known speech samples of the same speaker were excerpted from the same phonetic contexts, and 10 attempts where they were excerpted from different contexts. Statistically significant differences in subject performance for same and different context tasks indicated that the file card system does not substantially minimize the effects of contextually caused spectral variation. The magnitude of error rates suggests that it cannot be used for either population reduction or identification.

HH2. Speaker Recognition and Verification using Linear Prediction Analysis. M. R. SAmbur, Bell Telephone Laboratories, Whippany, New Jersey 07981.-The speaker recognition and verification effectiveness of a set of 92 measurements was examined. These measurements included the formant structure of vowels, the duration of certain speech events, the dynamic behavior of the formant contours, various aspects of the pitch contour throughout an utterance, formant bandwidths, glottal source "poles," and pole and zero locations during the production of nasals and strident consonants.
Linear prediction methods were employed in the analysis, and a probability of error criterion was devised to evaluate the speaker-characterizing potential of the measurements. The experimental speech data were collected during five different recording sessions, the last session being $3 \frac{1}{3}$ years after the original recordings. The measurements that were found most useful were related to the nasals, certain vowel resonances, certain temporal attributes, and average fundamental frequency. A speaker identification experiment using only the five "best" measurements resulted in only one error in the identification of 11 speakers for 320 test "utterances." [This work was part of a doctoral thesis at the Massachusetts Institute of Technology.]

HH3. Techniques for Objective Speaker Identification. Geroge D. Hair and Thomas W. Rekieta, Texas Instruments, Incarporated, P.O. Box 5621, Mail Station 939, Dallas, Texas 75222.-A technique has been developed and tested for identifying from voice recordings which, if any, of a number of possible speakers corresponds to a particular object speaker recording. Segments of the same set of selected phonemes by the object speaker and all suspect speakers are Fourier transformed. These phoneme spectra, and features derived therefrom, are utilized to make measures of voice similarity. Experiments with voice recordings by 140 men have yielded estimates of the probability of correct identification as a function of the number of suspects for both forced-choice and open-choice tests. Identification reliability has also been related to number of phonemes, number of utterances, and number and type of features. Open-choice decision reliability of $99 \%$ or better for small numbers of suspects was demonstrated. Another decision procedure, whereby decisions are made in only $95 \%$ to $99 \%$ of cases, can reduce error probabilities essentially to zero. 
HH4. Talker Differences as They Appear in Correlation Matrices Derived from Spectra of Continuous Speech. K.-P. LI AND G. W. Hughes, School of Electrical Engineering, Purdue University, West Lafayetle, Indiana 47907.-Previously, we have shown that stable estimates of correlation matrices derived from the outputs of bandpass filters can be obtained from about $30 \mathrm{sec}$. of continuous speech [K.-P. Li, G. W. Hughes, and A. S. House, J. Acoust. Soc. Amer. 46, 1019-1025 (1969)]. The present study describes the measurement and properties of intertalker and intratalker variability among these matrices. Three measures of the difference between two matrices were investigated, average absolute difference, Euclidian distance, and correlation between matrices. All measures of difference are consistently less for intratalker data than for intertalker comparisons. For our population of 30 talkers, the distribution of these differences shows only about $1 \%$ overlap to confound absolute identification of a given talker. The effects of processing and sample size are discussed, and applications to talker identification and normalization schemes suggested.

HH5. Analysis-by-Synthesis Recognition of Spoken Integers Using Prosodic Information Alone. J. Allen and Y. D. Willems, Research Laboratory of Electronics and Department of Electrical Engineering, Massachusetts Institute of Technology, Cambridge Massachusetts 02139.-A formal syntax for the spoken integers between 0 and 999 can be written using syllables as terminal elements, and which also utilizes word and syllable juncture as well as spoken variations (e.g., three versions of the lexical item "and") of some of these terminals. A match routine is then used to generate all possible syntactic strings from left to right. As each terminal is generated, a comparison is made of the syntactic structure with the syllabic and junctural correlates determined from the waveform envelope and pitch contour. In this way, a running likelihood score is generated for each possible terminal string as it builds up from left to right. This technique returns a small number of possible utterance candidates with a high degree of correctness. These results are obtained without use of any segmental information.

HH6. The Use of Prosodic Features to Derive Word and Syllable Boundaries. Y. D. WILleMs and J. ALLEN, Research Laboratory of Electronics and Department of Electrical Engineering, Massachusetts Instituse of Technology, Cambridge, Massachusetts 02139.-Spoken integers between 0 and 999 (e.g. "three hundred and seventy two") were recorded by four speakers. For each utterance, a measure of the waveform amplitude (called the envelope) and pitch period estimates were recorded and plotted. It is shown that these two correlates can be used to predict word and syllable boundaries within each spoken integer. These junctural correlates also reflect the semantic structure of the integers, so that both multiplication and addition operations are represented in the spoken number. Each boundary is also assigned a likelihood factor. These junctural determinations are determined without any use of segmental information (such as spectral estimates), and are directly related to the structure of the utterance.

HH7. An Experimental Technique for Establishing Lexical Variants by Rule in Automatic Recognition of Continuous Speech. C. C. TAPPERT AND N. R. Dixon, IBM Corporation, Thomas J. Watson Research Center, Yorktown Heights, New York 10598.-The primary task in phonetic-to-lexical translation is to develop procedures which, from a pragmatic point of view, provide the necessary paths for changing an acousticcontinuum reality into a series of discrete standard-orthographical forms, which, at the present time, exist only as theoretical abstractions. Implicit in this problem is the assumption that words exist acoustically, even though complexly, in a many-to-one mapping. For automatic recognition, a technique is required that will provide the variety that is to be accounted for in this many-to-one mapping. Since words are known to be highly variable with regard to their phonetic constituents, it is necessary to tie a representation system to some unit which is smaller than the word itself, but larger than the individual phonetic unit. In the present system, it was tentatively decided to attempt variant generation based on units generally corresponding to the syllable. While present results are not conclusive, it appears viable to continue the development of this approach. Whether or not a within-word unit can be used to characterize all interword phenomena has yet to be determined.

HH8. Some Problems in the Derivation of a Phonetic Referent for Evaluation and Lexical Representation in Automatic Recognition of Continuous Speech. N. R. Dixon and C. C. TAPPERT, IBM Corporation, Thomas J. Watson Research Center, Yorktown Heights, New York 10598.-One of the basic problems in Automatic Recognition of Continuous Speech exists relative to the representation of talker performance. Phonetically, the problem results in a requirement at the lexical level for either "narrow" transcriptions or a variety of "broad" transcriptions for representing any word. If pattern classification procedures are utilized in acoustic analysis, the former presents many problems involving inventory size. More importantly, a question arises concerning the appropriateness of the referent employed in performance evaluation. For speech, the referent-a description of what the talker actually produced rather than intended-is, even with a highly trained phonetician, many times difficult to obtain. It is clear that a single "ideal" transcription for each lexical item is inappropriate. Traditionally, in speech recognition work involving phonetic classification, a single transcription is provided for each of the words to be recognized. Systems have been proposed in which partial representation of "phonemes" may provide sufficient information for more sophisticated, i.e., context dependent, processing at a later time. However, it is not clear that these systems will be able to account for some of the dissimilatory, assimilatory, and/or ideolectical variants that a phonetician would certainly use in describing talker performance.

HH9. An Automatic Method for Computer Recognition of Continuous Speech. R. Byron Purves and William J. Strong, Department of Physics and Language Research Center, Brigham Young Universily, Provo, Utah 84601 .- An automatic speech recognition system for syntactically acceptable units of continuous speech has been implemented on a digital computer. The input speech to the system is carefully spoken by a single cooperative male speaker. A primary segmentation is attempted by delineating the boundaries of fricatives, stops, and nasals. The remaining vocalic sections are subsegmented on the basis of short-time phoneme identification. The principle identification procedure uses cepstrum matching; however, auxilliary use is made of zero crossing and slope change rates for fricatives, and apparent place of articulation for stops. Three phoneme choices arc assigned to each segment. In order to recognize the utterance, successive phoneme strings are generated from these choices until one is found which satisfies the lexical and syntactic constraints. The lexical constraint requires that the phoneme string consist only of "phonemicon" (phonetic dictionary) entries and the syntactic constraint requires that the word string satisfy a simplified English syntax. A relatively simple syntax checking algorithm has been devised for this purpose, which, although it admits some nonsense structures, imposes a severe constraint on allowed word strings. 
HH10. Use of Syntactic Segmentation and Stressed Syllable Location in Phonemic Recognition. WAyne A. LEA, Mark F. Medriss, and Toby E. Skinner, Univac DSD, P.O. Box 3525, St. Paul, Minnesota 55165.-Automatic speech recognition is expected to be more successful when syntactically related information is incorporated into early stages of recognition. Phonemic decisions, in particular, are expected to be more accurate and less ambiguous when contextual information is considered. A computer program has been developed for detecting boundaries between syntactic constituents from fall-rise fundamental frequency $\left(F_{0}\right)$ contours in connected speech. Another program detects some stressed syllables from $F_{0}$ contours, intensity contours, and timing information. Resulting boundary detections and stressed syllable locations are used by a preliminary method for estimating distinctive features of some phonemes in connected speech. Vowel and consonant recognition is attempted first in th" stressed syllables. Other readily detected segments, such as coronal unvoiced strident fricatives, are also found. Detected constituent boundary positions are compared with stored information about boundary positions, and are used both to choose candidate sentences independently and to help clarify phonemic results. Results for a limited sentencerecognition task are reported, and extensions to more versatile sentence recognition from syntactic segmentation, stressedsyllable location, and distinctive features estimation are suggested.

HH11. An Analysis of Wavefunction-Derived Speech Recognition Features. LARry L. Pfeifer, Compuler Systems Lab, University of California, Santa Barbara, California 93106.Based upon a wavefunction representation of wide-band filtered speech (five fixed-frequency bands), recognition features can be computed pitch synchronously and then later combined at the phoneme level. The pitch synchronous wavefunction frequency data at times acts as a formant tracker and at other times does not. The phoneme features from wavefunctions cluster much the same as equivalent formant features but not necessarily at the same locations in feature space. For certain types of sounds (e.g., /ao/), the detailed description provided by the wavefunction parameters can be useful in distinguishing one from the other. These and other relationships between formant and wavefunction based recognition features will be discussed.

HH12. A Model for Word-Level Conversion of Arbitrary Text to Speech. J. Allev, Research Laboratory of Electronics and Department of Electrical Engineering, Massachusetts Institute of Technology, Cambridge, Massachusetts 02139.While the acoustic correlates of linguistic structures above the word level are of great importance in speech synthesis, it has not been possible to develop a comprehensive theory for these effects. At the single word level, however, the present model shows how any English word can be converted to IPA symbols with stress marks sufficient to drive a phonemic synthesis-byrule algorithm. Words are either partitioned into constituent morphemes, or processed by a large set of letter-to-sound rules. The latter rely on the lexicon to contain high-frequency exceptions, and assume that all polymorphemic words have been split up into their constituent morphs. Both monomorphemic and polymorphemic lexical stress rules are applied, and morphophonemic rules provide low-level adjustments. The complete process provides a partial model for native speakers reading aloud, as well as the means to test current linguistic phonological theories.
HH13. A Theory on the Characteristics of Pitch and Formant Frequency Transitions in Human Speech. R. M. Doublier, Synthesis Research Group, Acoustic Phonetics Laboratory, University of Southern California, Los Angeles, California 90007.-Perceptual experiments have shown that the characteristics of fundamental frequency (pitch) and formant frequency variations usually represent a more important acoustic cue than those of the formant amplitudes. Lnfortunately, however, the exact nature of these frequency transitions is far more subtle. If the speech production process is viewed as a closed-loop feedback control system, application of modern control theory techniques yields a rather interesting model for the vocal-tract control function. This system may be viewed in terms of the familiar phase-locked loop. The loop filter model, which appears to fit both theoretical considerations and the empirical (sound spectrograph) results, is the so-called "imperfect integrator," with transfer function $F(s)=(s+a) /(s+b)$. A discussion of this proposed model, including samples of synthetic speech, is presented.

HH14. Preliminary Model of Tone, Intonation, and Segmental Duration in Serbo-Croatian. Edward T. Purcell, Linguistics Program, Universily of Southern California, Los Angeles, California 90007.-The paper will describe a 600 statement FORTRAN IV subprogram for the synthesis-by-rule of simple statements in Serbo-Croatian, a Slavic tone language. The model receives an input phoneme string, calculates the duration of each segment in the string, computes a set of fundamental frequency values corresponding to the sentence intonation, and then modifies these fundamental frequency values to reflect the influence of word tone. Several factors affect the calculation of segmental duration, e.g., number of segments in the string, number of syllables in the word, accented versus unaccented syllable, type of accent, voicing characteristics of adjacent consonants, and others. Word tone is calculated as a deviation from the previously defined sentence intonation. The paper will be accompanied by slides and tape-recorded samples.

HH15. Computer-Assisted Phonetic Analysis Techniques for Large Recorded Corpuses of Natural Speech. ANDRE MALtcot, Phonetics Research Facility, University of California, Santa Barbara, California 93106.-A system of computer programs has been developed for creating, from surreptitiously recorded conversations, data files containing phonetic transcription, intonation and stress patterns, allophonic variations, grammatical functions, demographic information such as the age and occupation of each speaker, psychological factors such as degree of formality, emotional state and intellectual level of subject matter, and paralinguistic factors such as syllabic rate, loudness, etc. The system also includes programs for locating phonetic patterns, expressed in a language readily understood by phoneticians with no computer training, and for analyzing them statistically, either for the corpus as a whole or as functions of any of the nonlinguistic or paralinguistic variables mentioned above. These techniques have initially been applied to 50 half-hour recordings of conversations with members of the Paris "establishment," revealing a number of phonetic laws hitherto unsuspected. The method is now ready and available for the study of the phonology of other languages, and other applications have appeared. One particularly promising possibility is the establishment of patterns of phonetic habits for a given number of individuals for voice verification purposes. 


\title{
Session II. Engineering Acoustics IV : Underwater Transducers; Measurements, Arrays
}

\author{
RoBert J. BobBer, Chairman \\ USRD/NRL, Orlando, Florida 32806
}

\section{Contributed Papers (10 minutes)}

\begin{abstract}
II1. Plane-Wave Simulator in a Mechanically Driven Standing Wave Hydrophone Calibrator. L. A. Abragnaro and B. B. BAUER, CBS Laboralories, Stamford, Connecticul 06905. A method has been developed to provide plane-wave operation in the Mark III pressure gradient hydrophone calibrator [Wide Range Calibration System for Pressure Gradient Hydrophones, B. B. Bauer, L. A. Abbagnaro, and J. Schumann, J. Acoust. Soc. Amer. 51, 1717 (1972)]. Since this calibrator uses a mechanical drive rather than a transducer drive to establish the standing wave field, conventional time delay techniques could not be employed. Rather, two loudspeakers were used in conjunction with the calibrator drive to achieve a plane wave at the center of the tank. A simple operating procedure is described for using the plane-wave mode, and some experimental results are presented.
\end{abstract}

II2. Equivalent Sound Pressure Levels of Different PressureGradient Hydrophones at Low Frequencies due to Turbulent Flow. S. P. LEE, The Magnavox Company, Fort Wayne, Indiana 46804.-Assuming turbulent pressure fluctuations near a hydrophone to be a substantial contributor to the acoustic low frequencies, the low-frequency response of a pressure-gradient hydrophone with different shapes and sizes is studied. It is demonstrated that the predicted equivalent sould pressure level at low frequencies can be reduced by changing the shape and/or size of the hydrophone. The result of the study shows that a pressure-gradient hydrophone with spherical shape exhibits $4-10 \mathrm{~dB}$ reduction in the equivalent sound pressure level as compared with a cylindrical shape at a frequency of $10 \mathrm{~Hz}$.

II3. A Reverberant Chamber Facility for Sound Power Measurements in Air or Water. W. K. BLAKE, Naval Ship Research and Development Center, Bethesda, Maryland 20034.A reverberant chamber for use with both air and water media is described. The chamber is used to investigate the acoustic coupling of simple vibrating structures under conditions of light or of heavy fluid loading. Calibration was effected by measuring $60-\mathrm{dB}$ reverberation times in $\frac{1}{3}$-oct bands; decay times of $2 \mathrm{sec}$ in air and $0.2 \mathrm{sec}$ in water were observed. These broad-band results were cross checked in water by measuring the spectrum of acoustic pressure in the chamber generated by a calibrated hydrophone source. Sound pressure levels measured in narrow $(50-\mathrm{Hz})$ bands varied $\pm 3 \mathrm{~dB}$ spatially in the air-filled chamber and $\pm 5 \mathrm{~dB}$ spatially in the waterfilled chamber.

II4. A Reverberant Water Tank for Underwater Measurements. B. G. WATTERs, Bolt Beranek and Newman Inc., Cambridge, Massachuselts 02138.-This paper describes the water-filled tank used at BBN for measuring the radiatednoise power of underwater sources. The tank has centerline dimensions of $32 \times 23 \times 14$ ft and contains $11000 \mathrm{ft}^{2}$. Its lowest resonance frequency is $220 \mathrm{~Hz}$; the lowest usable $\mathrm{f}$-oct band is centered at $320 \mathrm{~Hz}$ - low enough for direct measurements on full-size torpedoes. The tank can accommodate a large torpedo, full-scale sections of sonar domes and similar constructions, and suitably scaled models of larger structures. The ambient noise is low, near Knudsen SSO. The wall absorption coefficient is small (less than $5 \%$ at $1 \mathrm{kHz}$ ), producing very long reverberation times (up to $5 \mathrm{sec}$ ). A soundabsorbing treatment was developed to adjust the reverberation time to about $\frac{3}{4} \mathrm{sec}$ at all measurement frequencies. A multiple-hydrophone incoherent array is used to reduce the sampling error. The residual error due to the finite number of samples and to finite tank volune has been evaluated and compared to theoretical estimates. The facility is also used for ordinary pulsed "free-time" measurements.

II5. A Low-Frequency Technique for the Underwater Calibration of the Individual Elements of a Line Hydrophone Array. J. F. Zalesak and W. J. TrotT, Acoustics Division, Naval Research Laboratory, Washington, D. C. 20390.-The calibrator consists of three coaxial internally radiating piezoceramic cylinders with their ends and outside surfaces shiclded. These piezoceramic cylinders terminated with coaxial metal tubes form a feed-through calibrator when suspended in an open, water-filled trough. The two outer piezoccramic cylinders produce the sound which is monitored by the central piezoceramic cylinder. The calibration procedure consists of comparing the output from a line hydrophone element located inside the central piezoceramic cylinder with the output of the piezoceramic cylinder. The purpose of the water-filled metal tubes is to provide sufficient mass reactance in order to retain a usable sound pressure level at low frequencies. The upper frequency at which this procedure is valid is determined by the lowest resonant frequency of the piezoceramic cylınders. Pertinent design considerations of the calibrator are presented, and the technique for measurement is evaluated using a calibrator as described above.

II6. Calibration Accuracy for Small Standard Hydrophones. J. E. BluE, Underwaler Sound Reference Division, Naval Research Laboratory, Orlando, Florida 32806.--When a standardizing laboratory issues a certificate for a standard, it attributes to that standard a numerical value together with a statement about the uncertainty of this value. In the measurement of the sensitivity of an unknown hydrophone performed mac, most activities will claim an accuracy of $\pm 1 \mathrm{~dB}$. Usually no confidence limits are specified, and the accuracy is an educated guess based on experience. This paper discusses the careful "free-field" reciprocity calibration of two small standard piezoelectric hydrophones in a stable environment and the use of these hydrophones in evaluating the calibration performance of six of the established calibration facilities at the Underwater Sound Reference Division of the Naval Research Laboratory. The results of these measurements show that, for the most part, accuracy of the facilities is well within 
the $\pm 1 \mathrm{~dB}$ commonly quoted and is probably within $\pm 0.3 \mathrm{~dB}$ with $95 \%$ certainty at some of the facilities when calibrating small standard piezoelectric hydrophones.

II7. A New Underwater Microphone for Swimmer's Masks. L. A. Abbagnaro, J. Schumann, and B. B. Bauer, CBS Laboratories, Stamford, Connecticut 06905.-A new underwater microphone for use with swimmer's masks was developed. This unit features an equalized fluid-filled internal structure, which renders it pressure insensitive, permitting use at all diving depths. The transducer response is controlled mechanically rather than acoustically and exhibits no change with pressure variations. The new microphone is small enough to fit into most diving mask cavities and makes use of its pressure gradient design to minimize unwanted mask speech coloration. Performance data in free-field and in a typical diving mask were obtained for this device. [Work was supported by the U. S. Navy Explosive Ordance Development Facility.]

II8. An Electrical Load for Simulating a Transducer in an Array. W. C. Nowlin and J. J. Truchard, A pplied Research Laboratories, The University of Texas at Austin, Auslin, Texas 78712.--Individual transducer elements of large sonar arrays are subject to electrical impedance changes as a result of acoustical interactions between neighboring driven elements. In order to transmit acoustic power, the elements are driven individually or in groups by power amplifiers. When a prototype amplifier is constructed, an entire array of transducers and amplifiers may not be available for testing the amplifier. A passive electrical dummy load was constructed to have impedance characteristics equivalent to the particular type of transducer that a prototype current-source switching amplifier was to drive. An active port was added to the electrical dummy load to change the amplifier's load impedance at all frequencies of interest, thereby simulating different positions in a transducer array in which more than one element is driven. A digital computer program was written to calculate the drive voltage to be applied to the active port to obtain the desired impedance at a given frequency. The dummy load design technique is described. The dummy load impedance characteristics are compared with those of the transducer loads to be simulated; agreement is shown to be very good. [This work was sponsored by Naval Ship Systems Command.]

II9. Designing a Reflecting Surface Array of Compliant Tubes. G. A. Brigham, New London Laboratory, Naval Underwater Systems Center, New London, Connecticut 06320.A rather detailed model that considers multiple radiation coupling of compliant tube arrays has been developed and utilized for the purpose of designing several arrays. The model was generated by an iterative extension of the static solution to a uniformly loaded oval shell, and includes multiple crosssectional bending modes as well as multiple radiation modes, although the latter restricts the model to tubes with an elliptical cross-section. No account is taken of bending in the direction of the tube's cylinder axis, since the maximum correction this introduces to the cross-sectional flexural wavenumber is a term on the order of $\left(3 H C_{1} / S C\right)^{2}$ compared to unity, with $H$ the wall thickness, $C_{1}$ the shell material's longitudinal velocity, $C$ the acoustic velocity in water, and $S$ the cross-sectional midsurface circumference. As this factor is typically of order $5 \%$ or less, it is ignored. Numerical results for a 10-tube array are compared with experimental data for verification of the computer model. In addition several computer-evaluated array reflectivities are shown for different tube configurations. Finally, both center frequency and band- width are displayed as functions of array parameters. [Work performed under contract with NUSC.]

II10. Transducer Sound Dome Interaction. H. L. Pond, New London Laboralory, Naval Underwater Systems Center, New London, Connecticut 06320, and R. W. HoY, Newport Laboratory, Naval Underwater Systems Center, Newport, Rhode Island 02840. - The acoustic interaction of a transducer and a surrounding sound dome is analyzed by a modified separation of variables method. The theory is described for transducer and dome of general shape. If they are concentric spheres, the method reduces to classical separation of variables. Perhaps the simplest example that uses the general theory in a significant way is obtained if transducer and dome are concentric spheres but the thickness of the dome varies with position. Numerical results are given for this case. For the calculations the velocity at the transducer is phased to a plane wave and its magnitude multiplied by a constant to adjust for a fixed transducer power output, which is taken as that for the no-dome case. The results show that, with a dome that has a thickness increasing uniformly from a minimum value $A$ in the direction of the main radiated beam to a maximum value in the opposite direction, the directivity index and main beam magnitude are increased several decibels in comparison with results for a dome of constant thickness equal to the average of $A$ and $B$.

II1. Self and Mutual Acoustic Radiation Impedances for Two Coplanar Unbaffled Disks. A. L. VAN BuREN AND B. J. KING, Naval Research Laboratory, Washington, D.C. 20390.The self and mutual acoustic radiation impcdances for two coplanar unbaffled disks are calculated using an eigenfunction expansion in terms of oblate spheroidal wave functions. Terms representing outgoing waves from both disks are included. One disk is assumed to be vibrating with a rotationally symmetrical normal velocity distribution, and the other disk is assumed to be stationary. The determination of the expansion coefficients from these boundary conditions is facilitated by the use of an addition theorem which expresses spheroidal wave functions with reference to one coordinate frame in terms of spheroidal wave functions with reference to a second coordinate frame. Numerical results for the special cases where the vibrating disk is either oscillating uniformly or vibrating uniformly on only one side are presented and discussed.

II12. Calculations and Measurements of the Arially Symmetric Extensional Vibrations of a Circular Disk with a Concentric Hole (A Free-Flooded Ring). J. A. Sinsky, Naval Research Laboratory, Washington, D.C. 20930.-A complete mathematical model of a free-flooded ring transducer must predict the ring's surface velocity distribution when the forces acting on the ring are known. The acoustic radiation from a radially oscillating cylindrical ring is a function of its surface velocity distribution. In particular, the farfield radiation on the axis of the ring is a sensitive function of the difference between the inside and outside radial velocities and the axially directed edge velocity. Accelerometer measurements in air were made on a number of aluminum rings of varying thickness oscillating in axially symmetric extensional vibrations, and the experimental results are compared with the twodimensional theory of Gustafsson and Kane for high-frequency extensional vibrations of elastic isotropic plates. The results indicate that the theoretical analysis is adequate to predict the surface velocity distribution of a "thick ring." Comparisons are also made between the theoretical analysis and "back of the envelope" approximations commonly used by transducer engineers for an elastic ring's surface velocity distribution. 


\title{
Session JJ. Noise Control
}

\author{
M. J. CROCKer, Chairman
}

\author{
School of Mechanical Engineering, Purdue University, West Lafayette, Indiana 47907
}

\section{Contributed Papers (12 minutes)}

\begin{abstract}
JJ1. Noise Reduction of a Consumer Electric Clock. W. M. Viebrock* aNd M. J. Crocker, Ray W. Herrick Laboratories, School of Mechanical Engineering, Purdue University, West Lafayette, Indiana 47907. - The noise from a consumer electric clock was studied. The clock was placed in an anechoic room for sound pressure level analysis to determine the noise sources, which were found to be impactsat the electric motor rotor bearing and impacts between gear teeth of the rotor pinion and the first reduction gear. Study of the clock case revealed that case resonances were being excited by the impacts within the mechanism with resultant noise radiation. Modifications to the case to increase damping were successful in reducing the noise associated with these resonances, although a different damper must be devised for production feasibility. Noise reductions were also obtained by modifications to the clock motor itself including reduction in magnetization of the rotor; use of softer rotors and first reduction gears; reduction in the inertia of the first reduction gear; and increase in load applied by a friction drag to the gear train. [This work was supported by Westclox Division, General Time Corporation, LaSalle, Illinois.]
\end{abstract}

* Present address: Lockheed Missile \& Space Co., Sunnyvale, Calif.

JJ2. The Effect of a Biphase Lubricant on Induction Motor Bearing Noise and Stability. Douglas Mustek, University of Houston. Department of Mechanical Engineering, Houston, Texas 77004, and Jefrery O. Beauchamp, Mallay Tool Service, Houston, Texas 77021 . - A specially constructed singlephase induction motor was used to study the effect of a biphase (liquid-solid) lubricant on the stability of and noise from its bearings. It is shown that the stability of a full-film bearing was improved when a biphase lubricant was used. The biphase lubricant was an SAE 20 oil with from $5 \%$ to $20 \%$ (by volume) to $1-\mu$ fluorinated ethylene propylene (FEP) particles in suspension. A small reduction in bearing noise was observed. This was associated with the corresponding stabilizing effect of the FEP-loaded lubricant.

JJ3. An Investigation of Internal Combustion Engine Silencing Using Reflection and Absorption Phenomena. A. MuKhERJEE AND W. MitcheLl, Department of Mechanical Engineering, Tennessee Technological University, Cookeville, Tennessee 38501 . -An investigation has been made to determine the most influential parameters for efficient silencer design. These parameters lead to new insights into silencer operation containing both reflection and absorption phenomena, where damping is found to be effective at lower frequencies. Hence, objectionable deep exhaust tones of engines at frequencies of about 80-100 $\mathrm{Hz}$ can be significantly reduced. Efficient and economic silencing of engine exhaust noise can be applied to a large number of internal combustion engine installations including 2- and 4-cycle engines-e.g., cars, buses, trucks, locomotives, lawn mowers, chain saws, and motorcycles. Two important factors were considered simultaneously, i.e., the aerodynamic criterion (which specifies the maximum permissible average pressure drop through the silencer at a given temperature and mass flow) and the geometrical criterion, which specifies max- imum allowable volume and restrictions on shape. Results of this study are: (1) Identification of parameters influential to silencer design. (2) A better understanding of silencer operation incorporating reflection and absorption phenomena. (3) A more efficient overall silencer system. (4) Lower noise levels associated with internal combustion engine operation.

JJ4. Economics of New Plant Sites Based on Acoustical Considerations. Allan M. TEPLITZKY, Office of Environmental Affairs, Consolidaled Edison Co. of New York, Inc., Nezw York, New York 10003.-Environmental noise at the property line due to an industrial operation is one element that determines the minimum site area requirements for a new plant. Residents in communities surrounding a plant hear the noise created within the plant that is attenuated by the building and the size of the buffer zone between the plant and the community. In suburban and rural areas where the ambient background noise may be low, sites for heavy industry plants become exceedingly large if insulated metal type siding is used for the building walls to achieve a satisfactory sound level at the property line. When large sites are not available, or if the price of land is high, the site area can be considerably reduced by the use of masonry or multilayered insulated metal types of siding. For a plant with an interior noise level of $90 \mathrm{~dB} A$, the site area for a plant with masonry siding may be less than $10 \%$ of the site area for the same plant with insulated metal siding. The use of high sound transmission loss walls for plant buildings necessitates that noise from other sources such as fans, ventilating system, steam vents, etc., be reduced to meet the property line sound criterion. This paper presents a graphical method for estimating plant site area and setback requirements based upon maximum allowable property line noise levels, plant noise levels, and type of building siding selected for the construction. In addition, a method is proposed for determining the economic break-even point for purchasing additional property as an acoustical buffer zone versus the cost of improving the sound transmission loss of the building siding.

JJ5. The Radiation Resistance of a Simply Supported Beam. J. Y. Chung and M. J. Crocker, Ray W. Herrick Laboratories, School of Mechanical Engineering, Purdue University, West Lafayetle, Indiana 47907.-Convenient approximate solutions for the modal radiation resistance are presented for a baffled simply supported beam that is acoustically narrow. The results compare very well with those obtained by numerical integration, which are considered to be exact. The results calculated from the equations presented have been compared with the existing approximate solutions [R. H. Lyon and G. Maidanik, "Power Flow Between Linearly Coupled Oscillators," J. Acoust. Soc. Amer. 34, 623-639 (1962)]. It is seen that in most Irequency ranges the results presented offer better accuracy than those referenced. The approximate solutions presented are derived from an exact series solution of the modal radiation resistance. Three equations are derived for three different regions, namely, above-coincidence, belowcoincidence, and about-coincidence regions. For mode numbers higher than six, the equations for above and below coincidence practically cover the entire frequency range. 
JJ6. Evaluation of Noise Control for the World Trade Center PATH Terminal. F. M. KessLl" ". Lewis S. Goodfriend $\&$ Associales, Morristown, New Jersc'y "17960.-Track vibration isolation in the form of "tie-in-po-".:et" construction, a highly acoustical absorbent metal pan ceiling construction, and other noise control features were used at the newly built World Trade Center PATH Terminal Noise measurements were made at numerous positions at the Church Street PATH Terminal prior to its discontinuence as a terminal and at the World Trade Center PATH Termiı il just after its completion. The results of these surveys iulicate an improvement in passenger comfort due to the reduction in plitform and concourse noise levels. These data were tulylyed and compared as to noise criteria (NC), statistical $A$-weighted noise level, and statistical octave-band spectra. A noise level criterion of $L_{10}=75 \mathrm{~dB} A$ for passengers waiting at the platform was developed and compared with noise levels measured at the two terminals and at a typical mir!iown street location. Testing of the track lubrication systen, vas also accomplished.

JJ7. First Steps toward Noise Reduction of a Fly Shuttle Loom. Paul D. Emerson anIl George Georgopoulos, School of Textiles, North Caro. Siate University, Raleigh, North Carolina 27607.-Several : :ple and inexpensive loom modifications were evaluated is ans of reducing noise at the source in fly shuttle looms. Noise from a Draper model $X$ loom 40 in. wide and running at 160 picks per minute was analyzed before and after rodification. Spectra were determined both with and without a shuttle. Reductions of $2 \mathrm{~dB}$ in all weighted scales were achieved.

\begin{abstract}
JJ8. A Tape Recorder for Use With Sound-Level Meters. Eugene R. Martenex, General Radio Company, Concord, Massachusetts 01742.-A tapc recorder designed specifically for use with sound-level meters is described. Important considerations of recording from sound-level meters are disc' = - ed, including calibration, dynamic range, crest factor, frequency response, and futter. Particular attention is given to the recovery of recorded sound-level range One solution is to use a second record channel and tape track to record tones representing the sound-level meter range. Four bridge " $T$ " oscillators, multiplexed in a binary format, generate the encoding signal. The oscillators convert to filters during playback, selecting tones, which when detected, actuate a light emitting diode digital display. Encoding of tones is accomplished manually or automatically from sound-level meters that have an encoding switch as part of the attenuator selector. Convenience of a cassette recording format, and the si, ile setup and operate procedure make the recorder ideally suited for field use.
\end{abstract}

JJ9. Spatial and Temporal Distribution of Sound Pressure Levels in Data Processing Installations. $W$. W. LANG, G. C Maling, JR., And R. E. Wise. IBM Acoustics Laboratory, Poughkeepsie, New York 12602.--The sound field in a data processing installation depends upon the locations of the sound sources, the sound power radiated by the sources, the acoustical properties of the room (includine its total absorption area, volume, and shane). and the I ickground noise levels in the room due to ex.ertial sources. Spatial and temporal variations in the sound field are observed in typical installations. The relative inportance of the individual ' yctors affecting the sound field is assessed. The results of thi -tudy show that a scale factor may be used to predict the SI's iI an installation from SPL data ohtainerl on individual morhines in a semi-anechoic room. Hence. seuti-anechoic rorn:' data are useful for predicting installati, n nolx t. 1 l: anc: is suiding the design of a particular data xce-i! .. - anlit $t$, as well as serving as a measure of soui. e'miss!. is i: rink the design and development of a new data processing 11 ıchine.
JJ10. Vibration Suppression in Wood Planer Noise Control. F. D. H ART and D. A. Ferioli, Center for Acoustical Studies, P. O. Box 5801, North Carolina State L'niversity, Raleigh, Narth Caralina 27607, and J. S. StEwart, Newman Machine Company, Greensboro, North Carolina 27403.-Board vibration has been identified as the dominant noise generating mechanism for a majority of wood planers. The effectiveness of compact enclosures is greatly diminished owing to board vibration, since a portion of boards of normal stock length is always outside of the enclosure. A vibration suppression system has been designed to operate inside of an enclosure to reduce the amount of vibratory energy transmitted outside through the boards being machined. The system developed and its effectiveness in reducing wood planer noise when used in conjunction with a compact enclosure is described in detail. [Newman Machine Company and NASA are acknowledged for support of this work.]

JJ11. Low-Frequency Sound Radiation Characteristics of Simply Supported Plates. James W'. Moore, General Molors Proving Grounds, Milford, Michigan, and M. J. Crocker, Ray IV. Herrick Laboratories, Purdue Liniversty, II est Lafayetle, Indiana.-Sound radiation from vibrating plates is important in many engineering problems. Theories for the sound power radiated by simply supported plates have been developed by Maidanik [J. Acoust. Soc. Amer. 34, 809-826, (1962)], Smith and Lyon [NASA CR-160 (1965)], and others. In this paper, lormulas have been derived which predict the sound power and farfield directivity pattern for a plate vibrating well below coincidence. It can be clearly shown that the sound power radiated is the same as for four monopoles located at the corners of the plate having equal strengths related to the plate vibration amplitude and a phase relationship depending on the type of mode excited. Similar formulas were derived for acoustic edge modes. The sound pattern for edge modes is that of two acoustic line sources. The sound power radiated by both corner and edge modes is found to depend strongly on the product of the wavenumber and the plate dimensions and on the type of mode. [Work supported by American Standard Research and Development Center, New Brunswick, New Jersey, and conducted when both authors were at the Herrick Laboratories.]

JJ12. Sound Radiation Characteristics of Simply Supported Vibrating Beams. James W. MOORE, General Motors Proving Grounds, Milford, Michigan, and Malcoly J. Crocker, Ray IV. Herrick Laboratories, Purdue University, Lafayette, Indiana. --Theories for the sound power radiated from a simply supported beam have been developed by Lyon and Maidanik [J. Acoust. Soc. Amer. 34, 623-639 (1962)], and others. In this paper, simple formulas have been derived which predict the sound power radiated and the farfield directivity pattern for an acoustically narrow beam. The results clearly show that a vibrating beam can be modeled by two interfering monopole sound sources located at the ends of the beam having equal strenyths related to the vibration amplitude of the beam and a phase relationship depending on the type of mode excited. To test this theory, directivity patterns were measured for a 1-ftlong simply supported beam vibrating at its resonance freyuencies. The directivity patterns for the first five modes were found to agree well with those predicted by the theory. [Work supported by American Standard Research and Development Center, New Brunswick, New Jersey, and conducted when both authors were at the Herrick Laboratories.]

JJ13. Noise Exposure Meter Performance-Results of an Evaluation Program. W. A. Leasure, JR., and Ronalo L. Fisher, Nalional Bureau of Slandards, ITashington, D. C. 20234. - As a result of the promulgation of occupational noise exposure regulations by the Federal government, there are a 
number of noise exposure meters on the market today that provide a measure of noise exposure integrated (with appropriate weighting) over a time interval. The National Bureau of Standards (under the sponsorship of the Environmental Protection Agency) has completed total system and component evaluations on a number of different commercial models of noise exposure meters. Overall system performance tests were used to evaluate the dynamic response of the system and the nature and accuracy of the time integration for increasing and decreasing, as well as stationary, signals. The following tests were conducted to evaluate subsystem performance: microphone response to sounds of random incidence, $A$-weighting network response, detector response and averaging time, and accuracy of the readout system. The rationale of the test procedure utilized, details of the measurement techniques, and results obtained are discussed.

Thursdar, 30 Nov'ember 1972

Burgundy East, 8:00 P.M.

\title{
Session KK. Graduate Workshop: Education in Acoustics
}

For the first time in the history of the Acoustical Society, a workshop, run by graduate students in in acoustic programs from five different schools, will be held. This workshop will devote itself to a discussion of the major areas of acoustics for the next decade. It will also discuss, from a prospective graduate's point of view, how these areas can best be incorporated into an academic curriculum. The workshop will attempt to present the pros and cons of specialization versus diversification within an acoustic program.

Friday, 1 DEcember 1972

Burgundy EAst, 9:00 A.M.

Session LL. Physiological Acoustics III : Pathways and Cortex

\author{
J. Donald Harris, Chairman \\ Medical Research Laboratory, Submarine Base, New London, Connecticut 06340
}

\section{Contributed Papers (10 minutes)}

LL1. Organization of the Posterior Colliculus. JOE C. Adams AND D. C. TEas, Center for Neurobiological Sciences and Communication Sciences Laboratory, University of Flarida, Gainesville, Florida 32601.- Internal organization of the posterior colliculus cut in the coronal plane is demonstrated in silverstained material in a variety of species. Within the central nucleus, predominant fiber paths cross dorsolateral to ventromedial with another group crossing at right angles directed in the dorsomedial to ventrolateral plane. Electrophysiological investigations in the chinchilla indicate that penetrations made with a microelectrode in the dorsolateral to ventromedial direction successively encounter neurons whose temporal patterns are quite similar. Tonotopic organization corresponds to that previously described in the cat. Penetrations made in the dorsomedial-to-ventrolateral plane reveal little consistent progression as the electode moves through the central nucleus. Results are described in view of previously described input to the colliculus.

LL2. The Effect of Interaural Phase at Different Frequencies on the Discharges of Cells in the Posterior Colliculus of the Chinchilla. D. C. Teas and J. C. Adays, Communication Sciences Laboratory, Department of Speech and Center for Neurobialogical Sciences, University of Florida, Gainesville, Florida 32601.- Some cells in the posterior colliculus with discharge latencies between 7 and $10 \mathrm{msec}$ (presumably in the central nucleus of the colliculus) show variation in their discharge rates with changes in interaural phase of the acoustic stimuli. These cells may show a characteristic interaural delay since their response rates can reach a maximum (or minimum) at the same interaural time difference for different acoustic frequencies. However, for frequencies about 2 act below all optimum characteristic delay frequency, optimal delays may produce opposite effects. That is, delays that were inhibitory at the higher frequencies may produce excitation at the lower frequencies, and delays that produced excitation at the higher frequencies may produce inhibition at the lower frequencies. Although our data support the idea of a temporal regularity in changes of discharge rate with interaural phase changes, the label, characteristic delay, may be too limited a descriptor for cells that respond to interaural time or phase differences.

LL3. The Effect of Stimulus Intensity at Different Interaural Phases on the Discharges of Cells in the Posterior Colliculus of the Chinchilla. D. C. TEAS AND JoE C. AdAMs, Communication Sciences Laboratory and the Center for Neurobiological Sciences, Universily of Florida, Gainesville, Florida 32601.-Binaural intensity functions for cells in the posterior colliculus can vary in slope and sensitivity depending upon the phase relations of the stimuli at the two ears. Interaural phase differences that produced low and high discharge rates were chosen as locations on a cell's phase function at which to examine its response to binaural intensity. The inhibitory or excitatory effect of phase angle is maintained across a wide range of intensity.

LL4. Patterns of Activity in Single Neurons of the Auditory Cortex in Unanesthetized Macaque Monkeys. J. F. BrugGE AND M. M. MERzENICH, Laboratory of Neurophysiology, University of Wisconsin, Madison, Wisconsin 53706, and Departments of Physiology and Otolaryngology, University of California at San Francisco, San Francisco, California 94122 Action potentials were recorded from single neurons in auditory cortex on the supratemporal plane in $\mathbf{1 0}$ hemispheres in nine monkeys (macaca mulatta). Each monkey was trained to sit quietly in a chair with its head restrained. Pure tones were delivered monaurally or binaurally through rubber ear molds that fitted tightly into each erternal auditory canal. When one ear is stimulated, the number of spikes and the firing pattern depend upon the position of the stimulus within the response area of the cell. For most neurons, spike count is a nonmonotonic function of SPL. Commonly, neurons discharge a train 
of spikes throughout a tone of several hundred milliseconds duration at suprathreshold levels at best frequency. A few cells adapt quickly and discharge only a few spikes at onset of the tone. Others are inhibited by the tone or are excited only at the termination of the sound. When tones are delivered binaurally, the number of spikes and their temporal order are very sensitive to small interaural differences in SPL. At low frequencies, spike count is a periodic function of differences in time of arrival of the stimulus at the two ears. Neurons can have a characteristic delay. Within a given penetration, phase-sensitive neurons with very similar best frequencies and spike-count functions can have very different firing patterns.

LL5. Variation of Excitability of Neurons in Primary Auditory Cortex in the Unanesthetized Macaque Monkey; Effects of Sleep and Body Movement. M. M. Merzenich and J. F. BrugGe, Departments of Physiology and Otolaryngology, University of California al San Francisco, San Francisco, California 94122, AND Laboralory of Neurophysiology, University of Wisconsin, Madison, Wisconsin 53706.-Microelectrode mapping experiments have been conducted to determine the position, size, and internal organization of the primary auditory field in the macaque. These experiments have revealed: (1) The primary field is strictly tonotopically organized, and the entire cochlear partition is represented within it. (2) The boundaries of the primary field can be defined on cytoarchitectonic as well as physiological bases. (3) The primary field is bounded by four other representational areas, and there is evidence for fields beyond these. Several of these secondary fields can also be delimited on cytoarchitectonic bases, and several appear to be tonotopically organized. Studies have been made of the responsiveness of single neurons in several of these regions in unanesthetized monkeys during stages of wakefulness and sleep. The state of the animal was monitored by the EEG and by close inspection over closed-circuit television. When the animal was awake and sitting quietly, neurons could be very responsive to pure tones though the number of spikes evoked could vary from one stimulus to the next. The responsiveness of many neurons was dramatically reduced during corpuscular body movement. Similarly, a neuron's responsiveness was often very much reduced during both slow-wave and REM sleep.

LL6. The Control of Complex Auditory Cortex Units in the Unanesthetized Monkey. J. MILleR, R. BEATON, AND T. O'CONNOR, Regional Primale Research Center, University of Washington, Seattle, Washinglon 98195.-Cortical cells exhibiting "complex" response patterns to acoustic stimuli have been studied in the unanesthetized and behaving nonhuman primate. The response of these cells consists of more than one temporally restricted component. For example, cells have been observed with on and off components that are either excitatory or suppressive, or some combination of these. In one sample of approximately 100 cells, $30 \%$ exhibited a complex response pattern. In at least $20 \%$ of these, the individual components of the response pattern have been found to vary independently with changes in frequency of tonal stimulation and/or intensity of the sound stimulus. Representative examples included cells with an on and off component in which each component had a different best frequency or cells in which the relationship of frequency of firing to intensity of stimulation differed for each response component. Such observations lead to questions concerning the coding of signal information in terms of temporal characteristics of response patterns, and the manner in which response characteristics of higher auditory cells may be structured and controlled. From our observations, it is suggested that independent cellular inputs contributed at points along the central auditory pathway control the firing characteristics of some cortical cells. Analogies may be drawn to the structuring of receptive field characteristics of cells in the visual system. [Research supported by NIH Grants.]
LL7. A Comparison of Averaged Evoked Potentials Recorded from Auditory Nerve Region and Brain Cortex in Waking Monkeys. Chester R. Wilpizeski and Richard P. MaioriELLO, Department of Otolaryngology, Thomas Jefferson University, Philadelphia, Pennsylvania 19107.--Squirrel monkeys were fitted permanently with rigid depth electrodes having tips localized in the auditory nerve region and with screw-type electrodes fixed on the surface of the parietal brain area ("vertex"). Summed records of pure-tone evoked potentials were recorded from both electrode sites using either a digital or an analog signal averager. Compared with cortical AERs, nerve evoked potentials showed greater amplitudes, greater resistance to habituation, less test-retest variability, and were characterized by less ambiguous waveforms at near-threshold levels. Acuity functions based on nerve AERs generally show greater sensitivity (lower detection levels) than those based on brain cortex responses.

LL8. Human Brainstem Electrical Responses to Acoustic Signals. R. Galambos, K. Hecox, S. Hillyard, T. Picton, G. FuRMan, AND M. LeIGH, Department of Neurasciences, University of California at San Diego, La Jolla, California 92037.Jewett and Williston [Brain 94, 681 (1971)], using conventional EEG scalp electrodes, recently demonstrated clickevoked electrical responses that appear during the first $10 \mathrm{msec}$ post-stimulus and evidently reflect activity in the auditory nerve and progressively higher brain stem structures. We will describe experiments aimed toward developing a clinical audiometric test based upon these electrophysiological responses. Using clicks delivered at rates of $20-75 / \mathrm{sec}$, and computer averaging of 2000 to 6000 responses, we have shown in 10 normal subjects that the wave labeled $V$ by its discoverers increases in latency from $6+0.5$ msec at a click intensity of approximately $60 \mathrm{~dB}$ SL to 8 or 9 msec at levels around $10 \mathrm{~dB}$ SL. The amplitude of wave $V$ also declines as a function of decreasing intensity. Brief tones generate similar functions. Evidence that these responses are generated by the brain, not by muscle contractions or other antifactual sources, will be presented.

LL9. Intensity Function for Cortical Potential Evoked by Electrical Stimulation of the VIIIth Nerve. RichaRD A. WALLOCH, Department of Otorhinolaryngology, University of Oklahoma Health Sciences Center, P. O. Box 26901, Oklahoma City, Oklahoma 73190.-The effect of intensity upon the amplitude of the cortical potential evoked by electrical stimulation of the contralateral VIIIth nerve in an anesthetized guinea pig was studied. Square pulses, $0.5 \mathrm{msec}$ in duration, were delivered through stainless-steel electrodes implanted in the VIIIth nerve. The stimulus intensity was randomly varied and the amplitude of the cortical potential was measured from a storage oscilloscope in a double blind manner. The amplitude at first rose sharply and then plateaued as the current intensity was further increased. The intensity function for electrical stimulation was different from that usually observed for acoustical stimulation in two respects. (1) For similar increases in stimulus intensity, a greater increase in the amplitude of the evoked potential was observed with electrical stimulation than with acoustical stimulation. This suggests the use of loud sound in behavioral transfer tests between acoustical and electrical stimulation of auditory centers. (2) Increases of stimulus intensity beyond that needed to produce plateau levels caused a reduction in the evoked potential amplitude with acoustical stimulation, but no reduction was observed with electrical stimulation. These data suggest that the reduction observed at high intensities of acoustical stimulation is due to a cochlear process, most probably the cochlear microphonic. [Supported in part by the National Institute of Neurological Diseases and Stroke and the Deafness Research Foundation.] 
LL10. Effects of Frequency and Phase Reversal on Early Components of the Evoked Cortical Response. S. ZERLIN AND R. F. NaUNTON, Otolaryngology, University of Chicago, Chicago, Illinois 60637. - The latency of the $\mathrm{Na}$ peak, a landmark of the early evoked response that occurs about 20 msec after stimulus onset, was studied with respect to (a) stimulus frequency and (b) phase reversal. Third-octave clicks (center frequencies $500,1000,2000$, and $4000 \mathrm{~Hz}$ ) were presented to normal-hearing listeners at sensation levels (SL.s) of $5,20,35$, and $50 \mathrm{~dB}$; systematic decrease in latency was seen between 500 and 4000 $\mathrm{H}_{z}$, approximately $2 \mathrm{msec}$ at $50 \mathrm{SI}$ and $5 \mathrm{msec}$ at $5 \mathrm{SL}$. The effect of phase change was studied with a $250-\mathrm{Hz}$ tone burst gated on at the axis crossing and off 3 cycles later; the first half-cycle of the tone burst began as either a condensation or a rarefaction ( $180^{\circ}$ phase change). Results showed that the $\mathrm{Na}$ response to the stimulus beginning as a rarefaction occurred about 2 msec earlier than the response to the stimulus beginning as a condensation. These results may be interpreted as illustrating the close relation between cochlear events and the early evoked cortical response.

LL11. Cerebral Asymmetry in Infants, Children, and Adults: Auditory Evoked Responses to Speech and Music Stimuli. Dennis L. Molfese, Department of Psychology, Southern Illinois University, Carbondale, Illinois 62901.-Adults process language and music sounds in different hemispheres of their brain (Kimura, 1967). This cerebral asymmetry is thought to occur during language development after 2 years of age (Lenneberg, 1967). However, recent research (Eimas, Siqueland, Jusczyk, and Vigorito, 1971) indicates that infants can categorically perceive speech sounds. It is likely that some cortical area has already specialized to process these sound. To determine if cerebral asymmetry is present, auditory evoked responses (AER) to speech syllables, words, music and speech noise were recorded from the temporoparietal language processing area Cohn, 1971) in the left hemisphere and from the corresponding area in the right hemisphere for three groups of 10 subjects-infants, children, and adults. AER waveforms were averaged and measured for amplitude changes between $N_{2}$ and $P_{1}$ wave components for each hemisphere. Verbal stimuli elicited greatest AER activity from the left hemisphere while the mechanical stimuli elicited the largest responses from the right hemisphere in all subjects. Furthermore, the degree of lateralization decreased with age. The results were interpreted as support for the notion that the brain is specialized to process language and music differentially from birth.
LL12. Effects of Long Onset Ramp on Auditory Evoked Potentials (AEP). K. N. Williams, L. F. Elfner, and G. G. BotHe, Department of Psychology. Florida Stale University, Tallahassee, Florida 32306.-Human event related potentials (ERP) were taken using averaging techniques to tonal pulses with systematically varied onset ramp. The rate at which the tonal pulse grows to full amplitude (ramp) was linear on both signal onset and offset. Auditory evoked potentials (AEP), were taken on seven naive college sophomores, with demonstrated normal hearing (ISO, 1964), for onset ramps of 50 , $150,500,1500$, and 5000 msec and a no-signal condition. Offset was 50 msec for all signal conditions. Equipment, calibration, between-subject variation, and noise considerations required that 64 trials be averaged to achieve useful (approx. 8:1) SIN ratios. Trials consisted of 5.05-sec signal condition to $45-\mathrm{dB}$ sensation level full amplitude and 9.95-sec intertrial interval. Visual analyses indicate that onset ramp affects offset AEP progressively less for all signal conditions as ramp duration increases. Onset ramp associated AEP is not apparent beyond the 150 -msec condition. Stepwise correlational analyses are being performed to clarify the apparent dichotomous onset/ offset results.

\section{LL13. Abstract withdrawn.}

Friday, 1 DeCEMber 1972

El DORAdo WEst, 9:00 A.M.

Session MM. Physical Acoustics V: Ultrasonic Spectroscopy

Edwin L. Carstensen, Chairman

Department of Electrical Engineering, University of Rochester, Rochester, New York 14627

\section{Contributed Papers (10 minutes)}

MM1. Concentration Dependence of the Visoelastic Parameters of Aqueous Glycerol. C. E. BELL, Naval Ondnance Laboratory, While Oak, Maryland, AND C. M. DAvis, Naval Research Laboratory, Washinglon, D. C. 20390.-The shear and longitudinal elastic properties of glycerol-water mixtures have been investigated by means of ultrasonics [Slie et al., J. Chem. Phys. 44, 3712 (1966)]. These data were used to estimate the relaxational parameters of pure water by means of extrapolations from low temperature. The resulting $0^{\circ} \mathrm{C}$ data were again extrapolated as a function of concentration to pure 
water. In the present investigation, Brillouin scattering has been used to minimize both the temperature and concentration extrapolations. The Brillouin and ultrasonic results are found to be in significant disagreement. The effect of this disagreement on the viscoelastic parameters of pure water is discussed.

MM2. Sound Absorption in Dimethyl Sulfoxide-Water Mixtures. W. M. MAdIGosky, Naval Ordnance Laboratory, Silver Spring, Maryland 20910.-Ultrasonic attenuation was measured in dimethyl sulfoxide mixtures over the frequency and temperature range $5-200 \mathrm{MHz}$ and $25^{\circ} \mathrm{C}$ to $-80^{\circ} \mathrm{C}$. At the higher temperatures the quantity $\alpha / f^{2}$ was found to be independent of frequency and in excess of the value calculated from the classical Stokes equation for shear viscosity. The excess attenuation can be attributed to a volume viscosity, which is two-thirds of the shear viscosity. At the lower temperatures $\alpha / f^{2}$ was found to be highly frequency dependent and less than that predicted by the Stokes equation. Furthermore, the data indicate that both shear and volume viscosities are relaxing with a single relaxation time. The low value of the excess attenuation observed at high tempeatures suggests that there are no specific hydrogen bonded complexes being formed. The dramatic decrease in $\alpha / f^{2}$ with increasing frequency at low temperatures seems to indicate the onset of a glassy state.

MM3. Ultrasonic Velocity Measurements in Liquids at High Temperatures and Pressures. JACEk JARZYNSKI, Naval Research Laboratory, Washington, D. C. 20390.-An apparatus is described for speed of sound measurements in molten metals and fused salts as a function of temperature and pressure. Problems encountered in extending the temperature and pressure range are discussed. The ultrasonic velocity data are used to determine the equation of state of the liquid investigated [L. Davis and R. Gordon, J. Chem. Phys. 46, 2650 (1967)]. Results are presented for the temperature range up to $500^{\circ} \mathrm{C}$ and pressure range up to $1000 \mathrm{~atm}$. However, using the apparatus described, such measurements may be obtained over a considerably larger pressure and temperature range than covered in this investigation.

MM4. Acoustic Velocity of Fluorocarbon Liquids. H. R. Feldman, Applied Physics Laboratory, University of I'ashington, Seattle, Washinglon 98195.-The semiempirical theories of Rao and Schaaffs, which relate the acoustic velocity in a liquid to its chemical structure, have been extended to include liquids containing fluorine atoms in the molecule. The measured acoustic velocities of nine fluorocarbon liquids were compared with the velocities predicted by the theories. The value of Rao's bond addendum [D. Sette, Ricerca Sci. 20, 102 (1950)] for the C-F bond, which minimizes the rms deviation from the measured values of acoustic velocity, is $114 \mathrm{~cm}^{2}$ $(\mathrm{m} / \mathrm{sec})^{1}$. Use of this value results in an rms deviation of $\mathbf{5 5 . 6}$ $\mathrm{m} / \mathrm{sec}$ for the rine compounds. If one eliminates the two acids (perfluoroacetic and $n$-perfluorobutyric), which one would expect to be associative, the rms deviation is $38.8 \mathrm{~m} / \mathrm{sec}$ for the seven remaining compounds. A single parameter fit for Schaaffs' raumerfuillung $C-F$ bond addenda [W. Schaaffs, Ergeb. exakt. Naturwiss. 25, 109 (1951)] results in an rms deviation of $46.0 \mathrm{~m} / \mathrm{sec}$, and a two-parameter fit in an rms deviation of $37.3 \mathrm{~m} / \mathrm{sec}$ for the nine liquids. Further measurements of acoustic velocity have been or will be made on other fluorocarbons to obtain better values for these constants. An understanding of the acoustic properites of fluorocarbon liquids is important because their unusually low acoustic velocities make them useful as refractive media in acoustics.

MM5. Propagation of Sound in Critical Binary Liquid Mixtures near the Critical Point. J. HunTer, L. MoLe, J. Dill, AND S. Vimilvanich, John Carroll University, Cleveland, Ohio 44118.--It is well known that very large attenuation of sound is produced in critical binary liquid mixtures as the critical temperature is approached, but there is disagreement whether this large attenuation is due to scattering or to true absorption. We have scanned the radiation emanating from a scattering cell containing a binary liquid mixture at several closely spaced temperatures near the critical temperature. Whereas there is a very large drop in the strength of signal at zero bearing angle, there is no consequent rise at any other angle, as there is in the case of true scattering (e.g., as has been experimentally found in scattering from air bubbles.). We conclude that the attenuation measured by previous observers should be assigned primarily to true absorption, and that the effect due to scattering is negligible. [Work supported by the Office of Naval Research, Acoustics Branch.]

MM6. Ultrasonic Wave Propagation in the Critical Temperature Region of $\beta, \beta^{\prime}$-Dichloroethyl Ether +Hexane. ElizAbeth C. Bains and M. A. Breazeale, Department of Physics, The University of Tennessee, Knoxville, Tennessee 37916.Ultrasonic attenuation and velocity measurements in the critical temperature region of the binary system $\beta, \beta^{\prime}$-dichloroethyl ether thexane are presented. This system has an upper critical solution temperature at $12.7^{\circ} \mathrm{C}$. Measurements were taken at frequencies between 1 and $9 \mathrm{MHz}$. The dependence of both attenuation and velocity on temperature and on frequency was investigated. The temperature dependence of the attenuation compares favorably with the theory of Fixman (J. Chem. Phys. 36, 1961 (1961); however, the frequency dependence does not. The frequency dependence of the attenuation cannot be fit by a function that is a single power of frequency. A velocity dispersion is observed in the critical region. [Research supported by the U. S. Office of Naval Research.]

MM7. Low-Frequency Ultrasonic Absorption in Triethylamine-Water Solution near its Critical Solution Temperature. S. S. Yun, Department of Physics, Ohio University, Athens, Ohio 45701.-The ultrasonic absorption at low frequencies $(0.3-4.5 \mathrm{MHz})$ in a triethylamine-water binary liquid solution has been measured through the critical solution temperature by means of the pulse echo method. For each frequency investigated, the absorption coefficient exhibits a marked maximum at the critical solution temperature similar to the results shown in the earlier work at $600 \mathrm{kHz}$ by $\mathrm{A}$. G. Chynoweth and W. G. Schneider [J. Chem. Phys. 19, 1566 (1952)]. The temperature dependence of the attenuation $\alpha / w^{2}$ in the single phase of the solution is examined in terms of Fixman's theory and compared with the earlier results obtained for higher frequencies [S. S. Yun, J. Chem. Phys. 52, 10, 5200 (1970)]. The frequency dependence of the absorption coefficient is discussed. [This work, performed at John Carroll University, was supported by a NONR contract, NSF grant, and an Ohio University research grant.]

MM8. Origin of the Relaxational Sound Absorption in Sea Water below $1 \mathrm{kHz}$. F. H. Fisher, Marine Physical Laboratory, Scripps Institute of Oceanography, University of California, La Jolla, California 92037, AND ERNEST YEAGER, Robert Bressel, and John Miceli, Case Hestern Reserve Universily, Cleveland, Ohio 44106.-From long-range sound transmission data, Thorp [J. Acoust. Soc. Amer. 42, 270 (1967)] concluded that sea water has a relaxation at $1 \mathrm{kHz}$ $\left(r_{r} \simeq 1.6 \times 10^{-4} \mathrm{sec}\right)$ in addition to the magnesium sulfate relaxation at $\sim 100 \mathrm{kHz}$. Laboratory measurements with the temperature-jump relaxation method confirm the existence of a relaxation of the proper time $\left(\tau_{r}=1.5 \times 10^{-4}\right.$ at $\left.9.7^{\circ} \mathrm{C}\right)$ and identify this relaxation with the boric-acid-borate system in sea water. No relaxation has been observed in the range $10^{-5}$ $10^{-1} \mathrm{sec}$ in synthetic sea water, made up without borates or boric acid. Pressure shock measurements in boric-acid-borate solutions also reveal a relaxation in the proper time domain. The slow relaxing process is believed to involve the $\mathrm{B}(\mathrm{OH})_{3}$ 
$-\mathrm{B}(\mathrm{OH})_{4}^{-}$reaction coupled to fast reactions involving the other buffer systems (e.g., $\mathrm{CO}_{3}{ }^{2-}-\mathrm{HCO}_{3}{ }^{-}$) in the sea water. The complete analysis of this relaxation in sea water is complicated by these faster chemical processes. [Supported by Office of Naval Research.]

MM9. Vibrational Relarational in $\mathrm{CO}_{2} / \mathrm{O}_{2}$ Mixtures. H. E. Bass and F. Douglas Shields, Department of Physics and Astronomy, The University of Mississippi, University, Mississippi 38677. - The acoustic absorption in mixtures of $\mathrm{CO}_{2}$ and $\mathrm{O}_{2}$ has been measured for $90 \%, 80 \%$, and $70 \%$ concentration of $\mathrm{O}_{2}$ at $600^{\circ} \mathrm{K}$ and $450^{\circ} \mathrm{K}$ and $80 \%$ and $10 \%$ concentration of $\mathrm{O}_{2}$ at $300^{\circ} \mathrm{K}$. The absorption has been analyzed to extract the rate of energy transfer between the vibration of the bending mode of $\mathrm{CO}_{2}$ and the $\mathrm{O}_{2}$ virbation and the rate at which $\mathrm{O}_{2}$ deexcites the bending mode of $\mathrm{CO}_{2}$ in a v-t process. [Work supported by the Office of Naval Research.]

MM10. Ultrasonic Absorption Measurements in Single Organic Crystals. A. E. Victor, H. E. Altman, Jr., AND R. T. Beyer, Department of Physics, Brown University, Providence, Rhode Island 02912.- Two sets of measurements are reported of the ultrasonic absorption coefficient in single organic crystals. In benzene, the measurements were made with longitudinal waves in the frequency range $6-38 \mathrm{MHz}$ and over the temperature range $170^{\circ}-250^{\circ} \mathrm{K}$. Similar measurements over the same temperature range are reported for crystalline nitrobenzene for frequencies $6-62 \mathrm{MHz}$. A newly designed seedholding crystal growth tip greatly aided the growing of the single crystals of nitrobenzene. Present absorption theories predict absorption proportional to the square of the frequency, over the ranges covered in these experiments. In the case of benzene, however, there appears to be a relaxation peak in the range 15-20 $\mathrm{MHz}$ for propagation along the $a, b$ axes. The propagation along the $c$ axis is more complex. The experimental results for both crystals are compared with pertinent theory. [This work was supported in part by the Advanced Research Projects Agency, Department of Delense, and the National Science Foundation.]
MM11. Static and Dynamic PVT Measurements on $\mathrm{B}_{2} \mathrm{O}_{3}$ Glass Using an Acoustic Densitometer. Ronert D. Corsaro, Naval Research Laboratoly, Washington, D. C. 20390.-An acoustic densitometer has been used to determine the volume of a $\mathrm{B}_{2} \mathrm{O}_{3}$ glass sample over a wide range of temperatures and pressures, and the corresponding thermodynamic volume derivatives have been evaluated. At temperatures near the glass transition $\left(280^{\circ} \mathrm{C}\right)$, the dynamic volume relaxation corresponding to a pressure perturbation ("pressure jump") was also observed. Under such conditions, both the dynamic and static values of the compressibility may be evaluated, along with the apparent spectrum of relaxation times. These properties are then compared with predictions based on models of the glassy state.

MM12. The Equation of State of Water and Seawater as Determined from Sound Velocity Data. Rana A. Fine, Dong Pisg Wang, and Frank J. Millero, Rosenstiel School of Marine and Atmospheric Science, University of Miami, Miami, Florida 33149.-Using the high-pressure (0 to 1000 bars) sound-velocity measurements of Wilson (1960), an equation of state for PVT properties of water and seawater has been developed based on a modified Tait equation of the form

$$
\frac{V^{\circ}-V^{P}}{V^{\circ} P}=\frac{1}{B+A_{1} P+A_{2} P^{2}},
$$

in which $V^{\circ}$ is the specific volume at atmospheric pressure, $V^{P}$ the specific volume at pressure $P$, and $B, A_{1}$, and $A_{2}$ are functions of temperature and salinity. The equation has been fit with a maximum deviation in compressibility of $.0 .01 \times 10^{-0}$ $\mathrm{bar}^{-1}$ for seawater and $0.02 \times 10^{-1} \mathrm{bar}^{-1}$ for pure water. The data from this equation will yield specific volumes that show the excellent consistency of Ekman's (1908) measurements with the sound data. The results for pure water show a definite shift in Kell and Whalley's (1965) measurements and the superiority of the PVT data generated from the sound data to direct experimental measurements.

Friday, 1 December 1972

EMPIRE ROOM, 9:00 A.M.

Session NN. Effects of Noise

D. C. GaSAwAy, Chairman

U. S. Air Force School of Aerospace Medicine, Brooks Air Force Base, Texas 78235

\section{Contributed Papers (10 minutes)}

NN1. Review of Noise Impact Aspects of Environmental Impact Statements. Joel M. GaRrelick, Cambridge Acoustical Associates, Inc., Cambridge, Massachusetts 02138.-The noise impact aspect of a number of Environmental Impact Statements has been reviewed for projects within the New England Region. The projects include transportation facilities, e.g., highway and airport constructions, as well as standard building construction. Different levels of effort were apparent. These ranged from complete surveys of ambient levels plus detailed predictive schemes to a few cursory remarks. Various acceptability criteria and analyses were employed. However, a number of generalizations can be made. Finally, means for improving efficiency of preparation and presentation of these statements are suggested. [This work was partially performed under contract to the Environmental Protection Agency, Categorical Programs Division, Boston.]

NN2. Subjective Rating of Broad-Band Noises Containing Pure Tones. Pierre Goulet and T. D. Northwood, Division of Building Research, National Research Council of Canada, Otlawa, KIA ORO, Canada.-For rating the noise of broad-band sources the custom is to utilize $A$-weighted levels, but there is no consensus on the subjective importance of pure-tone components that may be present. This study was to provide some direct evidence on this point. Nine subjects participated, individually, and the procedure was to have the subject match pairs of noises by adjusting the level of one of them. Two experiments used a modified random noise to 
which a pure tone in the range $250-2000 \mathrm{~Hz}$ could be added. The first experiment involved a simple comparison in a quiet environment. The second involved similar noises but with the addition of a local "wanted" sound in the form of music, and the subject matched noises from the viewpoint of "intrusiveness." A third experiment was similar to the first but utilized actual machine noises as the basic spectra. All three experiments indicated that the $A$-weighted level remains an adequate (slightly conservative) rating number even when pure tones are present.

NN3. Improved Perceived Noise Computational Algorithm. W. J. Gruner, Teledyne Brown Engineering Company, Huntsville, Alabama 35807.-An improved algorithm for the computation of the perceived noisiness of an acoustic pressure stimulus has been developed. The algorithm is presented along with the development logic and fit criterion. Coefficient determination is achieved using a constrained least-squares method. A statistical analysis of fit is presented for the derived algorithm as well as previously available methodology. The new algorithm is shown to improve accuracy from $26 \%$ to $36 \%$ depending upon amplitude and reduces computational storage requirements by $15 \%$. Improved accuracy is achieved at every applicable $\frac{1}{3}$-oct band. This algorithm was derived during psychophysiological criteria development for space shuttle system launch and static firing sites. [The development was funded under contract under the cognizance of the George C. Marshall Space Flight Center.]

NN4. Effect of Noise on Sleep. G. J. Thiessen, National Research Council, Ottarea, Canada, K1A OS1.-When appropriate account is taken of duration of the stimulus the present data on interference of sleep by noise seem to present a consistent picture. This picture indicates a "threshold" of interference which varies inversely with the duration of the stimulus, while for a given stimulus length the probability of a shift in sleep level is proportional to the stimulus level. The slope of this line seems approximately independent of stimulus duration. Thus, when all the available data are combined, they appear to contradict previous statements that for sonic booms and other acoustic stimuli of short duration there is practically no dependence on intensity once the threshold of sleep disturbance is exceeded. There is still no evidence that any appreciable habituation exists.

NN5. In-Ear Monitoring of Noise Exposure. A. J. BRAMmER AND J. E. PIERCY, National Research Council of Canada, Physics Division, Otlawa, Canada, K1A OS1.-The device described at the last meeting has been used to obtain a continuous record of the sound field at the base of the concha. When the data are transformed into an equivalent diffuse field that would exist at the center of the head, an individual's exposure to noise can be directly compared with existing criteria for hearing conservation that include effects resulting from intermittent exposure (CHABA). A record of sound level as a function of time, obtained during a representative work cycle, coupled with a knowledge of the noise levels generated by similar machines enables a decision to be made on economic grounds as to whether to modify the work cycle or the machine. The method has been applied to operators of skidders who were found, in general, to experience an exposure considered unacceptable by either CHABA or OSHA. The use of quieter than average skidders and minor restrictions in the work cycle produces a noise exposure that would be acceptable to CHABA.

NN6. The Effectiveness of Motorcycle Helmets as Hearing Protectors. R. T. Harrison, U. S. Forest Service, Equipment Development Center, San Dimas, California 91773.-Measurements of the at-ear noise attenuation provided by several popular motorcycle helmets were made. To do this, a probe microphone was placed in a subject's ear. He then operated a motorcycle through a test cycle, which included the motorcycle's entire performance range. He then donned a motorcycle crash helmet and repeated the test cycle. In each case the at-ear noise was recorded and analyzed. A comparison of the analyses revealed the attenuation characteristics of each helmet tested. In addition to helmet manufacturer, helmet size and type of eye protection fitted were varied. Three different subjects were used to establish differences from person to person.

NN7. $A$-weighted Sound Levels Measured within the Cockpits of 339 Fixed- and Rotary-Wing Aircraft. Donald C. Gasaway, U. S. Air Force School of Aerospace Medicine, Brooks $A$ ir Force Base, Texas 78235.-Acoustic noise levels measured within 339 aircraft are depicted for seven groups of fixed-wing aircraft and four groups of rotary-wing aircraft. These measurements are described using octave-band noise envelopes and flat and $A$-weighted sound levels. Also, differences between flat and $A$-weighted levels are identified for different types of aircraft. The noise spectra and weighted levels are reported from noise measurements obtained within the cockpits of various aircraft during conditions of normal cruise. Noise data are reported for 271 fixed-wing aircraft: 142 powered by reciprocating engines, 45 powered by turboprop engines, and 84 powered by turbojet or turbofan power plants; and for 68 rotary-wing aircraft: 28 powered by reciprocating engines and 40 vehicles mated to turboshaft engines. The purpose of this report is to provide insight concerning the acoustic character of noise exposures commonly encountered within aircraft.

NN8. Effects of Continuous and Intermittent Noise on Work Behavior. J. D. Repko and M. LoeB, Performance Research Laboratory, University of Louisville, Louisville, Kentucky 40208.-A synthetic-work approach was employed in an investigation of the effects of $90-\mathrm{dB}$ continuous and periodic 96-dB intermittent broad-band noise on human performance. Ten Navy and Air Force ROTC cadets worked the tasks of a multiple-task performance battery (MTPB) $12 \mathrm{~h}$ a week (in three, 4-h sessions) during a 4-week training period. Subsequently, they were required to work 4 days, 8 h per day on a split-shift schedule during the presence of noise, and finally, work 2 additional days on the same split-shif $t$ schedule under quiet conditions. Mean percentage of baseline performance (a composite index of performance) was enhanced by a periodic 96- $\mathrm{dB}$ intermittent noise, and the extent of the effect varied as a function of the number of tasks concurrently performed in the work situation. On the other hand, 90-dB continuous noise neither enhanced nor degraded performance. [Research supported by the U. S. Army Behavior and Systems Research Laborntorv, Army THEMIS Contract; and by U. S. Army Medical Kesearch and Development Command.]

NN9. Survey of the Noise Environments of 300 Small Industries in North Carolina. L. H. Royster, Center for A coustical Studies, North Carolina State University, Raleigh, North Carolina 27607, W. C. Creel, Safety Coordinator, North Caralina Department of Labor, Raleigh. North Caralina 27602, W. G. TномаS, Department of Surgery, University of North Caralina School of Medicine, Chapel Hill, North Caralina 27514, AND C. E. ScotT III, R. J. Reynolds Tobacca Company, Winston-Salem, North Carolina 27101.-A total of 300 industries were surveyed with respect to their respective noise 
environments. These industries generally employed less than 100 employees. The surveys were conducted by the North Carolina Department of Labor Staff. Pertinent information concerning number of employees exposed, percent of employees exposed in different company size ranges, etc., was also obtained during the survey. These results are presented.

NN10. Practical Considerations in the Establishment of Effective Hearing Conservation Programs. C. E. Scort III, R. J. Reynolds Tobacco Company, Winsion-Salem, North Carolina 27101, W. G. ThомAS, Department of Surgery, University of North Caralina School of Medicine, Chapel Hill, North Carolina 27514, L. H. Royster, Center for Acoustical Siudies, North Carolina State University, Raleigh, North Carolina 27607.-Procedures of developing, implementing, and maintaining an effective hearing conservation program are presented. Special emphasis is placed on the employee's part and the need to develop special techniques in order to ensure the program's success.

NN11. Techniques and Results of Audiometric Testing in Hearing Conservation Programs. W. G. Tromas, Deparlment of Surgery, University of North Caralina School of Medicine, Chapel Hill, North Caralina 27514, L. H. Royster, Center for Acoustical Studies, North Carolina Slate University, Raleigh, North Carolina 27607, AND C. E. ScotT III, R. J. Reynolds Tobacco Company, Winston-Salem, North Carolina 27101 -Audiometric testing is an integral part of a hearing conservation program. Newer techniques, which more closely approach the state-of-the-art, including computerized testing, and telephone line operation, are available for testing large numbers of people with results that are in good agreement with standard testing techniques. These techniques, as they apply to preemployment audiograms, initial testing, and annual retests, are discussed. A large number of audiograms from several major industries have been reviewed. The results of this review indicates that approximately $50 \%$ of the employees on the initial test show significant high-frequency hearing loss, approximately $10 \%$ of the employees show hearing loss severe enough to be seen by Clinics or physicians for further testing, and approximately $5 \%$ of the employees have to be retested because of inaccurate results. On annual retests, approximately $15 \%$ of the employees show additional hearing loss with hearing conservation programs in effect. Criteria and techniques for evaluating hearing conservation programs are suggested, along with criteria for evaluating additional hearing loss on annual retests.
NN12. Comparison of Selected Damage Risk Criteria in Judging Industrial Noise Environments. C. E. ScotT III, $R$. J. Reynolds Tobacco Company, Winston-Salem, North Carolina 27101, L. H. Royster, Center for A coustical Studies, North Carolina State University, Raleigh, North Caralina 27607, And W. G. Tномаs, Department of Surgery, University of North Caralina School of Medicine, Chapel Hill, North Carolina 27514. - A study was made of over 500 data samples for the purpose of comparing allowed exposure times using $85 \mathrm{~dB} A, 90 \mathrm{~dB} A$, and octave band spectra with the CНABA Working Group damage risk contours. The results of the study indicate that the use of octave band data spectra and the $\mathrm{CHAB}$ A Working Group damage risk contours should be allowed in lieu of overall $\mathrm{dB} A$.

NN13. Acoustical Testing of Potentially Hazardous NoiseProducing Toys. Marilyn A. Cadoff and Donald S. BlomQUIST, National Bureau of Standards, Washington, D.C. 20234. - In recent years, a great concern has been expressed for consumer protection and safety, especially for children. As an outgrowth of this concern, acoustical testing of potentially hazardous noise-producing toys has been carried out at the National Bureau of Standards for the Bureau of Product Safety (FDA). This talk will discuss the work that has been done on cap pistols and carbide cannons. The discussion will focus particularly on the development of a standard firing apparatus for testing paper caps, and on the problems encountered and the solutions arrived at for lesting carbide cannons.

NN14. Measurement and Analysis of Noise from Detonating Cord Applications. A. Viksne AND T. H. Logan, Engineering and Research Center, Bureau of Reclamation, Denver, Colorada, AND J. EAston, Martin Marietta Cement Western Division, Lyons, Colorado 80540.-A series of tests were conducted to measure the acoustical characteristics of 10 different types of commercially available detonating cord. Test measurements were made using impulse sound level meters, recorded on tape, and analyzed for amplitude and frequency content. A number of curves are presented showing the variation in the sound pressure level with explosive core load in grains per foot, and direction of the blast wave. Covered and uncovered cord were detonated and the SPLs compared. Values obtained from these tests are compared with noise criteria for hearing damage and community response to highintensity explosive noises. Results from sonic boom tests are used to correlate conmunity reaction to the noise from blasting.

\title{
Session OO. Speech Communication VII : Dichotic Encoding and Speech Perception
}

\author{
George Allen, Chairman
}

University of North Carolina, Chapel Hill, North Caralina

\section{Contributed Papers (7 minutes)}

001. Language Lateralization in a Case of Extreme Psychosocial Deprivation. Stephen Krashen, Victoria FromKIN, AND SUSAN CURTISS, Deparlment of Linguistics, UCLA, Las Angeles, California 90024, DAvid RIGLER, Children's Hospital, Los Angeles, California, ANd Sarah SpITz, UCLA Phonetics Laboratory, UCLA, Los Angeles, California 90024.Verbal and nonverbal dichotic listening tests were conducted on "Genie," a 15-year-old right-handed girl, who endured 10 years of extreme isolation from age 3 to 13 . When Genie was first admitted to the hospital there was little evidence of her having acquired any language. Her nonverbal dichotic results are not unusual; environmental sound perception appears to be normally lateralized to the right hemisphere. Her verbal dichotic listening results are singular in both direction and 
degree; she shows a left ear (right hemisphere) superiority to a degree matched only in split-brain and hemispherectomized subjects. This suggests that ear competition is taking place only between left ear contralateral and right ear ipsilateral pathways. These results are discussed in view of her steady but modest progress in language acquisition.

002. A Parallel between Degree of Encodedness and the Ear Advantage: Evidence from a Temporal-Order Judgment Task. Ruth S. DAy and James M. Vigorito, Haskins Laboratories, New Haven, Connecticut, and Department of Psychology, Yale University, New Haven, Connecticut 06510.Some speech sounds are more highly encoded than others. In acoustic terms, this means that they undergo more restructuring as a function of neighboring phonemes. In psychological terms, it may mean that special processing is required to perceive them. Stop consonants appear to be the most highly encoded speech sounds, vowels the least encoded, with other sounds falling in the middle. Stops, liquids, and vowels served as target phonemes in tests of dichotic temporalorder judgment (TOJ). A different syllable was presented to each ear with one leading by 50 msec, e.g., BAE(50)/GAE. Subjects reported which syllable began first. Ear difference scores were obtained by taking percent-correct TOJ on trials where a given ear received the leading stimulus and subtracting percent-correct TOJ on trials where the other ear led. Stop consonant pairs yielded a right-ear score, liquids a reduced right-ear score, and vowels a left-ear score. A rightear advantage in dichotic listening is usually interpreted as reflecting the operation of a special speech processing mechanism. The present data suggest that the highly encoded speech sounds required the services of this mechanism to a greater extent than did the less encoded sounds.

003. A Parallel between Degree of Encodedness and the Ear Advantage: Evidence from an Ear-Monitoring Task. James E. Cutting, Haskins Laboratories, New Haven, Connecticut, and Department of Psychology, Yale University, New Haven, Connecticut 06510.--Some phoneme categories undergo more acoustic variation in different environments than do others. The phonemes that undergo the most context-conditioned variation are said to be highly encoded. There appears to be an encodedness continuum, extending from the highly encoded stop consonants through the liquids and other sounds, and finally to the least encoded vowels. Stop-liquid-vowel nonsense syllables such as GLAE were used in a dichotic ear monitoring task. There were two possible phonemes in each class: $/ g /$ and $/ k /$ for stops, $/ l /$ and $/ r /$ for liquids, and $/ x /$ and $/ e /$ for vowels. Subjects reported only the syllable presented to a given ear for each block of dichotic trials. Primary analyses were performed on phoneme contrast trials, e.g., GLAE/KLAE for stops, GLAE/GRAE for liquids, and GLAE/GLEH for vowels. Data were scored in terms of percent-correct phoneme identifications for each ear. There was a large right-ear advantage for stops, a smaller right-ear advantage for liquids, and no ear advantage for vowels. The ordering of phoneme classes along the ear advantage continuum, therefore, was in parallel with the ordering of the same classes along the encodedness continuum.

004. Memory for Dichotic Pairs: Disruption of Ear Report Performance by the Speech-Nonspeech Distinction. RuTH S. Day, James C. Bartlett, and James E. Cutting, Haskins Laboratories, New Haven, Connecticul, and Department of Psychology, Yale Universily, New Haven, Connecticut 06510.When dichotic pairs are presented in rapid succession, the best strategy is usually to segregate the items by ear of arrival. If the stimuli fall into distinguishable classes such as letters and digits, then report by class is roughly comparable to that obtained by the ear method. In the present experiment, a stimulus class distinction markedly interfered with listeners' ability to use the ear report method. The classes were speech (/ba, da, ga/) and nonspeech (500-, 700-, and 1000-Hz tones). A trial consisted of three successive pairs of speech to one ear and nonspeech to the other. In separate blocks of trials, subjects reported the order of arrival for a given stimulus class (speech or nonspeech) or for a given ear (left or right). Performance was excellent for both types of report as long as all the speech went to one ear and all the nonspeech went to the other ear. However, when speech and nonspeech switched between the ears during a trial, class report remained excellent but ear report dropped substantially. The status of stimuli as speech or nonspeech, then, is a fundamental distinction, one that can reduce the effectiveness of the normally useful ear report method in dichotic memory tests.

005. The Right Ear Advantage for Speech Processing. Steve Herman, Program in Psycholinguistics, Universily of Michigan, Ann Arbor, Michigan 48104.-Spoken digits, words, CV syllables, and Morse code are recognized and recalled better by the right ear than the left; nonverbal sounds, such as melodies, tones, sonar beeps, and toilet flushes are processed better by the left ear than the right. In general, however, ear differences have been obtained with dichotic, but not monaural stimulation. To account for these findings, Kimura proposed that under dichotic stimulation the ipsilateral auditory connections are inhibited, thus giving the right ear "privileged access" to the language-dominant left hemisphere. Several findings involving monaural stimulation, verbal and nonverbal interference tasks, etc., are presented that indicate that Kimura's theory is inadequate. An alternate model based on interhemispheric transfer of information is proposed.

OO6. Dichotic Right Ear Advantage in Males and FemalesAges 5 to 13. C. I. Berlin, S. S. Lowe-Bell, L. F. Hughes, AND H. L. BerLin, Department of Otorhinolaryngology, Kresge Hearing Research Laboratory of the South, Louisiana State University Medical Center, New Orleans, Louisiana 07119.A total of 150 right-handed children (15 boys and 15 girls in each age group: $5,7,9,11$, and 13 ) listened dichotically to 60 pairs of precisely aligned nonsense syllables. All children had normal peripheral pure-tone sensitivity and monotic syllable intelligibility. A right ear advantage was seen even in children 5 years of age with no differences between the sexes as a function of age. The size of the right ear advantage and the nature of the phonetic errors, as well as the interactions between ear, age, sex, and phonemes will be presented. All effects will be discussed in terms of double corrects (where both items of the pair were correctly perceived) and single corrects (where only one item of the pair was correctly perceived).

O07. The Effect of Memory Load on the Right Ear Advantage in Dichotic Listening. Grace Yeni-Komshian and Joel GoRdon, The Johns Hopkins University, Baltimore, Maryland 21205 and Paul Sherman, Chicago Slate College, Chicago, Illinois 60621 . - The right ear advantage for reporting speech signals observed in dichotic listening tasks was further investigated to examine the effects of memory load on this phenomenon. The time interval between stimulus presentation and response was varied. In all experiments subjects went through a no-delay condition (immediate recall) and different delay conditions that sampled intervals between 5 and 15 sec. Normal adult listeners were stimulated with dichotic triads composed of one-syllable common English words. The first experiment consisted of a free ear-order recall task, while in the second experiment subjects were cued as to which ear to report from. The results from both experiments show an overall right ear superiority, which was most pronounced in the longest delay condition. There was a sharp drop in ac- 
curacy for both ears at about 5-6 sec delay. With increasing delay times, the number of correct responses for the left ear stimuli continued to decrease, while the number of correct responses for the right ear stimuli either leveled off or showed an increase. A third experiment investigated the effect of reducing memory load by providing the subject with a chart listing all the stimuli (chart condition). The effect of delay on the right ear advantage with no chart was replicated, but the right ear effect was not observed in any of the chart conditions. The absence of a right ear effect in the chart conditions was due to an increase in the accuracy of the left ear scores while the right ear scores remained at the same level of accuracy under chart and no chart conditions. These findings are interpreted to demonstrate that the right ear advantage is not solely a perceptual phenomenon but that the recall of verbal material is also lateralized and complements the perceptual asymmetry. [Supported by PHS Post Doctoral Fellowship and research grants from NINDS.]

O08. Perceptual Processing Time for Consonants and Vowels. D. B. Pisoni, Department of Psychology, Indiana University, Bloamington, Indiana 47401.-Perceptual processing time for brief CV syllables and steady-state vowels was examined in a backward recognition making paradigm. Subjects were required to identify a 40-msec target sound selected from either a consonant set (/ba/, /da/, /ga/), or a vowel set $(/ \mathrm{i} /, / \mathrm{I} /$, $/ \varepsilon /)$. The target sound was followed by a different sound drawn from the same set after a variable silent interstimulus interval. The second sound interrupted the perceptual processing of the target stimulus at short interstimulus intervals. Recognition performance improved with increases in the silent interstimulus interval. One experiment examined processing time for consonants and vowels under binaural presentation. A second experiment compared consonant and vowel recognition under both binaural and dichotic presentation. The results indicated that (1) consonants require more processing time for recognition than vowels, and (2) binaural and dichotic presentation conditions produce differential effects on consonant and vowel recognition. These findings have several implications. First, speech perception is not immediate, but is the result of several distinct operations that are distributed over time. Second, speech perception involves various memorial processes and mechanisms which recode and store information at different stages of perceptual analysis. [Research supported in part by grant to Haskins Laboratories from NICHD and a PHS Bio-Medical Sciences Grant to Indiana University.]

O09. Categorical and Noncategorical Modes of Speech Perception along the Voice Onset Time Continuum. JoAx House Lazarus AND D. B. PISONI, Department of Psychology, Indiana University, Bloomington, Indiana 47401.- Synthetic stop consonants varying in voice onset time (VOT) are generally perceived in a categorical mode. Listeners can discriminate two sounds only to the extent that the stimuli have been categorized as different phonetic segments. In the present study, native speakers of English were required to first identify and then discriminate stimuli which varied in VOT from $/ \mathrm{ba} /$ to $/ \mathrm{pa} /$. One group of listeners took a randomized absolute identification test, whereas another group took a sequential identification test. In the latter condition, stimuli along the continuum were presented in consecutive order. Half of the subjects in each group then took either an ABX discrimination test or a $41 \mathrm{AX}$ test. In the $4 \mathrm{IAX}$ test, two pairs of sounds were presented on each trial. One pair was always the same and one pair was always different. Subjects were required to judge which pair was the same, the first or second. Noncategorical perception of the voicing distinction was obtained only for the group of listeners who received both the sequential identification test and the 4IAX discrimination procedure. These findings suggest separate auditory and phonetic levels of processing in speech discrimination.

O010. The Verbal Transformation Effect: A Comparative Study of the Verbal Transformations of Phonetically Trained and Nonphonetically Trained Listeners. Norman J. LASS and Richarn M. Gasperivi, Speech and Hearing Sciences Laboratory, School of Medicine, West Virginia University, Morgantown, West Virginia 26506.-Continued listening to recorded repetitions of a stimulus has been found to produce perceptual illusory changes in normal listeners. This phenomenon was labeled by Warren [Brit. J. Psychol. 52, 249-258 (1961)] as the verbal transformation effect. The purpose of this investigation was to compare the reported verbal transformations of phonetically trained and nonphonetically trained subjects in terms of the number of forms and transitions elicited, number of repetitions of the stimulus prior to the subject's first verbal transformation, types of transformations reported, and exact forms employed. A total of seven auditory stimuli, representing variations in meaningfulness and phonetic complexity, were presented individually to 28 phonetically trained and 28 nonphonetically trained listeners. Results of their responses to the stimuli indicate that there are both similarities and differences between the two groups. The differences that exist are quantitative, but not qualitative, in nature. The phonetically trained group reported more forms and transitions, and required fewer repetitions of the stimulus prior to their first verbal transformations, than the nonphonetically trained subjects. However, both listener groups reported the same types of verbal transformations and, in many instances, the identical forms as well. Implications of these findings for future research are discussed.

OO11. Perception and Production of Stops in Bilinguals and Unilinguals. Alfonso Caramazza and Grace YenI-KoMSHIAN, The Johns Hopkins University, Baltimore, Maryland 21218, Edgar Zurif and EtTore Carbone, Sir George Williams University, Montreal, Canada-Cross-language studies have shown that voice onset time (VOT) is a sufficient acoustic cue to separate initial stop consonants into phonemic categories. The present study is an attempt to investigate further the efficacy of VOT as a linguistic cue by examining the perception and production of stop consonants in three groups of subjects: unilingual Quebec French, unilingual Quebec English, and bilingual French-English speakers. Perception was studied through a labeling procedure using synthetically produced stop tvowel syllables. Production was assessed through spectrographic measurements of VOT in word-initial stops. The six stop consonants of each language were investigated. The two groups of unilingual subjects had different perceptual functions and the bilinguals' responses were in an intermediate position. The production data indicate that VOT can separate between the English phonemic categories of $/ p, t, k /$ and $/ b, d, g /$, but this measure was not adequate for the Quebec French stop categories. In addition, the data show that language switching in bilinguals is well controlled at the production level but poorly controlled at the perception level. [Research supported in part by National Research Council of Canada and PHS research grant from NINDS.]

O012. A Multidimensional Analysis of the Phonemic Responses of Hard of Hearing and Deaf Subjects of Three Languages. Jeffrey L. Danhauer and Sadanand Singh, School of Hearing and Speech, Ohio University, Athens, Ohio 45701.-Twenty logatomes (CVCV) involving five vowels and 20 consonants were presented to 36 subjects, 18 hard of hearing and 18 deaf. Of the 18 subjects, six were speakers of English (American), six Serbo-Croatian (Yugoslavian), and 
six French (French). The logatomes were analyzed for both initial and medial consonants. The responses were recorded on magnetic tape and transcribed phonetically by three judges. The data were analyzed by IND-SCAL and reported in unique dimension for both stimulus and subject spaces. Twelve groups of similarity matrices mainly yielded two- and three-dimensional solutions. The interpretation of these dimensions were mainly voicing and sibilant. Additional features retrieved in some cases were nasality, fricative, and voiceless stop. Recovery of similar space for three language groups, two deviancy groups, and two positions reveal "universal" dimensionality and features of the consonants. Some features were more prominent at the initial place (sibilant and fricative), whereas others at the medial place (voicing and nasality). While hard of hearing and deaf subjects utilized voicing equally, sibilant, nasality, and fricative were utilized more by the hard of hearing and voiceless stop by the deaf subjects.
0013. Phonation and Loudness. Jared Bernstein, Phonetics Laboratory, University of Michigan, Ann Arbor, Michigan 48104. - Often when vowel loudness is measured, only SPL, duration, fundamental frequency, and formant frequencies are considered as causes of loudness. In the present study, differences in loudness were measured which apparently are a function of the mode of laryngeal vibration. Stimuli were constructed by having speakers produce a series of vowels at a single fundamental frequency and at several sound pressure levels (SPLs). The sustained vowel sounds were then electronically equalized for SPL and presented to subjects as pairs of isolated vowels. Differences in loudness were reported by subjects when asked to make paired comparisons. Vowels with equal duration, SPL, formant frequencies, and fundamental frequency were judged to be different in loudness as a function of the phonation quality associated with the vowels' position in the voice range of the speakers.

FRIDAY, 1 DECEMBER 1972

Dominion Room, 9:00 A.M.

Session PP. Aeroacoustics and Turbulence

Murray Strasberg, Chairman

Naval Ship Research and Development Center, Washington, D. C. 20001

\section{Contributed Papers (10 minutes)}

PP1. A State-of-the-Art Report on Aeroacoustic Testing in Conventional Wind Tunnels. R. E. A. ARNDT, Aerospace Engineering Department, Penn State University, University Park, Pennsylvania 16802, And D. A. Boxwell, U.S. Army Air Mobility Research and Development Laboratory, Moffett Field, California 94035.- The increasing attention given to aerodynamically generated noise brings into focus the need for quality experimental research in this area. To meet this need, several specialized anechoic wind tunnels have been constructed. In many cases, however, it is desirable to use conventional wind tunnels. Two basic problems are inherent in conventional facilities; high background noise and strong frequency-dependent reverberation effects. This paper reviews the known acoustic characteristics of several existing tunnels and presents new data obtained in a conventional $7 \times 10$ wind tunnel and a smaller $4 \times 5$ wind tunnel, which is convertible from a closed-jet to an open-jet mode with an anechoic test section. The data from the smaller tunnel serve as a guideline for evaluating design techniques involving a closed-jet and sandwich-wall construction. Model scaling parameters and data reduction techniques are also considered. It is concluded that existing conventional wind tunnels can be utilized for atroacoustic measurements. The overall technique, however, will require a careful balance of acoustic treatment, selection of proper model scaling, experimental technique, and data reduction schemes. Several suggestions are offered that are pertinent to a relatively large, low-speed wind tunnel facility.

PP2. Noise Sources and Room Acoustics of Closed Circuit Subsonic Wind Tunnels. I. L. Vér, Bolt Beranek and Nezuman Inc., Cambridge, Massachusetts 02138. - The noise aspects of subsonic wind tunnels have been studied experimentally on full-scale and model-scale tunnels. In wind tunnels without acoustical treatment, the driving fan and the boundary layer suction fan were found to be the dominant noise sources. The fan noise in the test section scales with the 6 th power of the airspeed. Using this scaling, one obtains a number, the acoustical merit rating, which permits one to compare the acoustical quality of different wind tunnels. Experimental results obtained in full scale as well as in scale models of closed circuit tunnels will be presented to illustrate the impulse response and spatial distribution of the sound pressure. Also, the special instrumentation required for obtaining valid results will be discussed.

PP3. Noise Control of Closed Circuit Subsonic Wind Tunnels. I. L. VÉr, Bolt Beranek and Newman Inc., Cambridge, Massachusetts 02138. - The noise control measures discussed will include the design of low-noise driving fans, the acoustical design of the exterior walls, and the sound absorbing treatment of strategically located interior wall surfaces. It will be shown how scale models can be used to predict the effect of noise control measures in reducing fan noise in the tunnel proper and sound power radiated to the outside.

PP4. Sound Diffracted by a Turbulent Boundary Layer Flow off a Sharp Edge. K. L. Chandiramani, Bolt Beranek and Nezman Inc., Cambridge, Massachusetts 02138. - The problem of diffraction of aerodynamic noise off the sharp edge of a rigid semiinfinite plate has received close attention recently. The approaches of Ffowcs-Williams and Crighton, dealing with scattering from a volume distribution of quadrupoles near the plate edge, have recently been recast by Chase with the aim of relating explicitly the magnitude of the scattered sound in the farfield with the wall pressure statistics characterizing a turbulent boundary layer (TBL) flow. Chase's treatment started with the known Green's function for the problem. In this paper, the same problem is treated in terms of diffraction off the plate edge, when the incident wave is pseudosound; it travels parallel to the plate surface with a velocity less than sonic and decays exponentially in a direc- 


\section{TH MEETING A ACOUSTICAL SOCIETY OF AMERICA}

tion normal to the plate. The resulting solution of the diffracted sound wave in the far field, when combined with Corcos' model of the TBL wall pressure statistics, yields good agreement with Chase's results. Comparison of these estimates with Hayden's data is made.

PP5. Nearfield Pressure in Perturbed and Unperturbed Turbulent Jets. G. L. BAREfoot and R. E. A. ARNDT, Department of Aerospace Engineering, The Pennsylvania State University, University Park, Pennsylvania 16802.-Consideration is given to the necessary nearfield pressure data necessary for predicting farfield acoustic radiation. A method for measuring two-point pressure correlations within a turbulent flow is developed and applied to three variations of a turbulent, axisymmetric jet: unperturbed, screen perturbed, and one containing a 10:1 ellipsoid in the potential core. Typical length scales for turbulent fluctuations are found to be smaller than equivalent velocity scales. The effects of a screen on the pressure field are significant. On the other hand, a body perturbed jet suffers a large increase in turbulence intensity but little change in the Reynolds stress and pressure field. Consideration is given to low-frequency roll-off problems and a limited frequency response. It is shown that meaningful data can be obtained in the range $1<x / D<7$ provided the phase lag of each probe is matched. It is also noted that there is an optimum jet velocity for a given facility in which acoustic contamination is minimized.

PP6. Wall Pressure Fluctuations at Smooth and Rough Boundaries under Turbulent Boundary Layers with Positive and Negative Freestream Pressure Gradients. Thомаs E. Burton, Massachusetts Institute of Technology, Department of Mechanical Engineering, Cambridge, Massachusetts 02139.Turbulent boundary layers were fully developed over either smooth or rough walls. Reynolds numbers based upon boundary layer thickness and freestream velocity are on the order 10 . Fluctuating pressures at the boundary were investigated within a local region, where a freestream pressure gradient was applied. The flow reequilibrated to the negative pressure gradient and was self-preserving, in the region of measurement. With a positive pressure gradient, the flow tended toward separation with increasing downstream distance and was not self-preserving. Two flush-mounted microphones provided pressure spectral densities and time-autocorrelations at single points on the boundary, as well as cross spectra and cross correlations from pairs of points. The influence on wall pressure of roughness is explained in terms of its effects on the mean velocity profile. Fluctuating pressure models, which incorporate a unique dispersion relation $c(f)$ (the phase velocity $c$ is obtained from the cross-spectral density) are found to be inapplicable to flows with a positive freestream pressure gradient. [Research supported by NSRDC.]

PP7. Laboratory Simulation of Spacecraft Acoustic Environment within a Cylindrical Enclosure. H. N. McGregor, Martin Marietta Aerospace, Denver, Colorado 80201.-Spacecraft integrity is verified by subjecting it in the laboratory to acoustic excitation, which simulates the vibroacoustic environment that occurs during launch, maximum aerodynamic pressure during exit from the earth's atmosphere, and the subsequent aerodynamic pressure fuctuation, which accurs during entry into the Martian atmosphere. Scale model tests were performed to determine if an enclosure approximating the shape of the spacecraft fairing could be used as an inexpensive test chamber. Tests were conducted with chambers having the configuration of the spacecraft fairing and a cylindrical shape. Results of the tests show that the acoustic environment can be replicated in a fairing shaped enclosure over $85 \%$ of the top surface and $95 \%$ of the bottom surface of the spacecraft. With a cylindrically shaped enclosure, they can be replicated over $95 \%$ of the upper and lower surfaces of the spacecraft. A low-pressure cold jet located in the lower half of the enclosure is used to simulate entry acoustics.

\section{PP8. Abstract withdrawn.}

PP9. Some Effects of Modal Coupling in the Transduction of Turbulent Pressure Fluctuations into a Closed Cavity. W. A. Strawderman, New London Laboratory, Naval Underwater Center, New London, Connecticut 06320. - The transduction of turbulent boundary layer pressure fluctuations into a closed, rigid walled, rectangular cavity via a fexible, thin plate is theoretically treated from a model approach. The turbulenceexcited vibration of the flexible plate induces pressure fields in the fluids internal and external to the cavity, which react on the plate and cause coupling of the plate modes. Since the plate modes excite the cavity modes, coupling of the plate modes results in coupling of the cavity modes. The forces on the plate resulting from coupling with the cavity may be interpreted as additional inertia or bending stiffness, and those resulting from coupling with the free field may be interpreted as additional inertia and resistance. The coupling forces cause changes in both the mode shapes and natural frequencies of the cavity.

PPI0. Abstract withdrawn.

PP11. Abstract withdrawn. 
PP12. Infrasound of 1 through $16 \mathrm{~Hz}$ Associated with Clear Air Turbulence. Eric S. Posmentier, Department of Meteorology and Oceanography, New York University, Bronx, New York 10453.-1- through $16-\mathrm{Hz}$ infrasound detected by a four-element array of microphones was recorded six times daily from April through June 1971. Four records were selected as representative of extremely low-amplitude conditions. Four other records were chosen as representative of highly coherent, strong-amplitude records. These latter four records all exhibited very high horizontal phase speeds, implying near-vertical incidence. A search was made for meteorological parameters which varied consistently with the acoustic data. It has been found that all four low-amplitude records were from periods during which nonturbulent conditions probably existed between altitudes of 6 and $12 \mathrm{~km}$. The four strong-amplitude records coincide with times of high probability of clear air turbulence. The turbulence judgments are based on the wind speed, the directional shear, and Richardson's number, calculated from nearby radiosonde data. It is concluded that 1 - through $16-\mathrm{Hz}$ infrasound may be radiated by clear air turbulence, and may be a basis for a remote passive detection system. If confirmed, this conclusion would have significant implications for both practical and theoretical problems associated with clear air turbulence.
PP13. Propagation of Waves of Acoustic Frequencies in Curved Ducts. W. Rostafinski, NASA-Lewis Research Center, Cleveland, Ohio 44135.-Propagation of long waves (very low frequencies) in curved ducts has been solved analytically and the results published [82nd Meeting of the ASA, J. Acoust. Soc. Amer. 51, 154(A) (1972)]. It was found that the basic mode of propagation is characterized by a wave pattern totally different from that known in a straight duct. To examine the propagation of shorter waves, in the acoustical frequency range, a systematic study of the pattern of higher modes has been done. Eact solutions up to the fourth mode are presented. Analysis applies to ducts with long and short radii of curvature of arbitrary width. The results are compared with propagation in a straight duct and important differences in the behavior of waves are noted. Analysis was made possible by the use of Bessel functions of the order $(n+1)$.

PP14. Acoustic Propagation in Ducts with Varying Cross Sections. Ali H. Nayfeh and Demetri P. Telionis, Engineering Mechanics Department, Virginia Polytechnic Institute and State University, Blacksburg, Virginia 24061.-The method of multiple scales was used to derive the equations that describe the spatial and temporal variation of the amplitudes and phases of acoustic modes propagating in slowly varying hard-walled or lined ducts. The analysis was carried out for rectangular as well as circular ducts. These equations were also derived from the conservation of energy. For largeadmittance or high-frequency modes, an approximate expression was obtained for the attenuation. This expression shows that all possible acoustic modes are attenuating. The results also show that varying the cross-sectional area can lead to elimination of some of the acoustic modes.

Friday, 1 DeCEMber 1972

Royal Room, 9:00 A.M.

\title{
Session QQ. Musical Acoustics
}

\author{
Ingo R. Titze, Chairman \\ Brigham Young University, Provo, Utah 84601
}

\section{Contributed Papers (12 minutes)}

QQ1. Mechanical Impedance Properties of a Toy Xylophone and Two Steel Drums. David S. Pallext, Ordnance Research Laboratory, The Pennsylvania State Universily, Universily Park, Pennsylvania 16802.-A series of informal measurements of the mechanical driving point impedance properties of a toy xylophone and two steel drums is described. The motivating factor for these measurements was to establish the feasibility of accurate identification of the fundamental pitches for the various segmented regions of the steel drum from mechanical impedance data. Complicating factors, which limit the accuracy of these measurements, are indicated. It is shown that interesting mechanical properties of the toy xylophone can readily be demonstrated. Successful measurements were conducted upon a large steel drum which indicate that: (I) the fundamental component of the free decay or the frequency for the lowest principal resonance is nominally a major third above the indicated pitch, but that (II) variations from this tuning of \pm 1 semitone are common in the lowest octave; (III) variations of \pm 3 semitone are common in the upper octave; and (IV) the subjective impression of tonal purity is correlated with the absence of modal degeneracy or coupling in the fundamental mechanical resonance.

QQ2. Acoustic Attenuators for Brass Wind Instruments. Perry Smith, John Stein, and Daniel W. Martin, D. $H$. Baldwin Company, Cincinnati, Ohio 45202.-The acoustical radiation from trumpets, Flugelhorns, trombones, and French and baritone horns has been greatly attentuated, without the adverse effects that conventional mutes have upon the natural timbre of the tone and the acoustical impedance at the player's lips. The new lightweight attenuators [Patent 3,555,956] are mounted upon the horns in contrast to a previous type [Patent 2,618,191] demonstrated at a Society meeting in 1947 by the present senior author. The absorptive part of the attenuator and the enclosed microphone lie just outside the open-end reflection region of the horn mouth, minimizing reaction upon the resonant horn and its modal frequencies. Listening to the enclosed microphone by earphones allows a performer to practice in relative privacy. A classroom of brass students so equipped can be taught individually, in groups or 
in ensembles by one teacher through a versatile communication system.

QQ3. Recent Studies of Wall and Air Resonances of a Violin. E. V. JANSSON, Department of Speech Communication and Institute of Optical Research, Rayal Institute of Technology $(K T H)$, S-100 44 Stackholm 70, Sweden.-Normal modes of the walls are studied by means of hologram and laser-speckle interferometry and their vibration patterns, frequencies, and $Q: s$ are registered. Resonance frequencies and corresponding $Q:$ s of the air volume enclosed by the walls of the sounding box are measured by especially designed electroacoustical equipment and a first image of the normal modes has been registered. The experimental findings will be presented and discussed.

QQ4. A Method for Visualizing the Eigenmodes of Free-Free Violin Plates. Carleen M. Hurchins, Catgut Acoustical Society.-Hologram interferometry has shown the shape of many of the eigenmodes of free-free violin plates. By hanging a violin plate horizontally from its four corners on rubber bands over a specially designed loudspeaker, and the speaker activated by a sine wave from an audiogenerator, it is possible to visualize readily some plate modes. This is done by using finely cut bits of aluminium that will not roll on the curved surface to make Chladni patterns as the plate is activated at its discrete mode frequencies. This is a particularly useful method of checking the contours and activity of the "ring mode" or plate tap tone, which has been found most effective in indicating where to thin the wood of violin plates before assembly. By combining this with our present plate tap tone method, which gives accurate check on frequency, amplitude, and damping of the "ring mode," we have a much improved method for controlling sound quality in finished instruments from violin to contrabass.

QQ5. The 1215-Cent Octave: Convergence of Westem and Nonwestern Data on Pitch Scaling. W. J. Dowling, Department of Psychology, UCLA, Los Angeles, California 90024.Ward [J. Acoust. Soc. Amer. 26, 369-380 (1954)] showed that Western listeners are extremely precise in making octave judgments with pure tones. Ward's listeners adjusted pairs of pure tones to subjective octaves that were slightly larger than a physical 2/1 frequency ratio (12 semitones or 1200 cents). Ward's data between 250 and $2000 \mathrm{~Hz}$ slowed mean subjective octave sizes of about 1215 cents. The validity of Ward's procedure for establishing a scale of musical pitch was dramatically reinforced by the agreement between scaled octave judgments and absolute judgments by one listener with absolute pitch. Observations made on the tuning of instruments in several nonwestern cultures show good agreement with Ward's result of 15 cents/oct sharpening. For example, reanalysis of Hood's [UCLA Ethnomusicol. Inst. Sel. Rep. 1, No. 1, 28-48 (1966)] data on the tuning of Indonesian gamelans shows a $15.36 \mathrm{cent} /$ oct sharpening. The results of all available precise measurements for widely divergent cultures are summarized. It appears that humans the world over make octave judgments with precision and that these deviate systematically from the $2 / 1$ ratio in ways that must be explained by the structure of the auditory system. The present results enhance the universal applicability of IVard's pitch-scaling methods.

QQ6. Precise Complex Tone Generation by Digital TableLookup Techniques. Stanley A. White, North American Rockwell Corporation, 3370 Miraloma Avenue, Anaheim, California 92803. - Complex arbitrary periodic waveforms may be generated by simple table-lookup means. An important tradeoff exists between the number of points of that waveform which is stored in the memory and the interpolation complexity. A simple frequency-generation technique is analyzed, and the attendant accuracy requirements (word length) are developed. Next, the relationship between the amplitude of the points stored to represent the waveform and its frequency spectrum are derived in a review of the discrete Fourier transform. The transfer functions (and frequency responses) of the buffers associated with the memory and the interpolator, as well as the transfer functions of the interpolators proper are derived. Shown also are the elfects on the frequency response of the generator system as a function of the number of interpolation points and the order of the interpolator. The mathematical model of the interpolator is in exact agreement with measured data. The system is easily mechanized by a read-only memory, an accumulator, adders, and simple logic. Applications have been to communications, sonar, vibration testing, and music synthesis.

Friday, 1 December 1972

El Dorado EAst, 9:00 A.M.

\title{
Session RR. Radiation and Scattering
}

\author{
George Chertock, Chairman
}

Naval Ship Research and Development Center, Washington, D. C. 20234

\section{Contributed Papers (10 minutes)}

RR1. On High-Frequency Scattering Methods: The Need for Error Bounds and Domains of Validity. R. NEW AND T. J. EIsLER, Acoustics Group, The Catholic University of America, Washington, D. C. 20017.--There exists an abundance of highfrequency scattering methods-but relatively little is known of their accuracy. Furthermore, the technical literature is significantly lacking in demonstrating the efficacy of one method of target scattering analysis versus another, and very few comparative analyses have been conducted-why? The Kirchholf, Keller, and geometrical optics (acoustics) methods are well known, and can be applied to arbitrarily shaped bodies. Comparative results are shown for the problem of scattering from a rigid sphere to demonstrate the differences between methods. Further comparisons to the more exact 
wave-harmonic and Watson series solutions emphasize the importance of establishing domains of validity for the approximate methods.

RR2. Comparison of Various Methods for Computing the Axially Backscattered Field of a Prolate Spheroid. J. Pijanowski AND R. New, Acoustics Group, The Catholic University of America, Washington, $D$. C. 20017.--It is difficult, if not impossible, to obtain error bounds on many solutions to highfrequency scattering problems. Consequently, comparisons between solutions can be very helpful in establishing domains of validity. The backscattered field from a rigid prolate spheroid, ensonified by a plane wave, is calculated using the Keller, Kirchhoff, Luneberg-Kline, and geometrical-optics methods. These results are compared to each other, and to the more exact wave-harmonic result. Finally, results from a finite element method, employing Schenck's CHIEF program, are compared. Limitations on the various methods are discussed.

RR3. Backscattering from a Finite Rigid Cylinder. T. J. EISLER AND R. New, Acoustics Group, The Catholic University of America, Washinglon, D. C. 20017. - The backscattering cross section of a finite rigid cylinder is calculated for plane-wave incidence at various aspects using both the Kirchhoff approximation and Keller's geometrical theory of diffraction. In the Kirchhoff method, the first term in the asymptotic expansion of the Kirchhoff integral in inverse powers of $k a$ is used, where $a$ is the radius of the cylinder, $k$ is the wavenumber of the incident radiation, and the length of the cylinder is assumed to be of the same order of magnitude as the radius. In Keller's method, only singly diffracted rays are considered. The calculations are compared with experimental results of Dunsiger [J. Sound Vibration 13, 323 (1970)]. In the special cases of beam and end-on aspect, there is specular reflection in the backscattering direction. In these two cases, the backscattering cross section is plotted as a function of $k a$ using higherorder terms in the asymptotic expansion of the Kirchhoff integral and multiply diffracted rays in the Keller method. These results are compared with the geometrical acoustics cross section.

RR4. The Radiation Patterns of Acoustic Multipole Sources in Motion. G. C. Gaunaurd, Naval Ordnance Laboratory, Silver Spring, Maryland 20910, AND T. J. EISLER, The Calholic University of America, Washington, $D$. C. 20017.-A study is presented of the radiated part of the pressure and velocity fields produced in free space by the arbitrary motion of point acoustic sound sources. Three types of multipole sources (monopoles, dipoles, and quadrupoles) are analyzed in detail, especially for the extreme cases of rectilinear and circular motions. Extensions to all the higher-order multipoles are also given. The solid angle distribution and the combined frequency and solid angle distribution of the radiated power are studied in further detail for point sources, lorces, and stresses in general arbitrary motion. Particularizations for the cases of rectilinear and circular motions are given, and the instantaneous radiation patterns are plotted in various cases. Some examples are worked out and some applications to the theory of sound radiation from rotating machinery are given as particular cases of the general theory. The total radiated power from each type of multipole undergoing each considered motion is also given. The analysis has applications when modeling the acoustic radiation produced by moving submerged bodies.

RR5. $k$-Space Formulation of the Acoustic Scattering Problem. Norbert N. Bojarski, Research Contractor and Consultant to the Department of Defense, 16 Circle Drive, Moorestown, New Jersey 08057.- The acoustic scattering problem is solved by means of a $k$-space formulation of the field equations, thereby replacing the conventional integral equation formulation by a set of two simultaneous algebraic equations in two unknowns in two spaces (the constitutive boundary condition being an algebraic equation in $x$ space). These equations are solved by an iterative method with the aid of the fast Fourier transform (FFT) algorithm connecting the two spaces, requiring trivial initial approximations. Since algebraic and FFT equations are used, the number of arithmetic multiply-add operations and storage allocations required for a numerical solution are reduced from the order of $N^{3}$ and $N^{2}$, respectively (for solving the matrix equations resulting from the conventional integral equations), to the order of $N \log _{2} N$ and $N$, respectively (where $N$ is the number of data points required for the specification of the problem). The advantage gained in speed and storage is thus of the order of $N^{3} / \log _{2} N$ and $N$, respectively. This method is thus considerably more efficient, and permits exact numerical solutions for much larger problems than possible with the conventional integral equation-matrix inversion method. The details and some numerical results of the application of this method to two- and three-dimensional acoustic scattering are presented.

RR6. Acoustic Radiation in the Near- and Farfield of an Annular Ring Using Impulse Response and Fast Fourier Transform Techniques. P. R. Stepanishen, General Dynamics/Electric Boat Division, Groton, Connelicut 06340.-An application of impulse response and fast Fourier transform techniques is presented to investigate transient and steady-state pressures in the near and farfield of a baffled piston with a specified velocity. As a specific example, and exact expression for the impulse response from an annular ring to an arbitrary point is presented. Time-dependent pressures in the field are expressed as a convolution integral involving the piston velocity and an impulse response. For harmonic fields, the pressure at a point is related to the Fourier transform of the impulse response at the point. The Fourier transform of the impulse response is evaluated exactly for on-axis points and points at large distances from the ring, and the results are seen to agree with the classical steady-state results. In general, the Fourier transform of the impulse response must be evaluated numerically and the use of fast Fourier transforms is proposed as a convenient method of evaluating the transform. The use of the approach to obtain nearfield pressure contours over a wide frequency range is also discussed and several numerical considerations are noted.

RRT. The Impulse Response and Acoustic Radiation Impedance of an Annular Ring in a Planar Baffle. P. R. StephanISHEN, General Dynamics/Electric Boat division, Groton, Conneclicut 06340 . - The acoustic radiation impedance of an annular ring in a rigid infinite planar baffle has been the subject of several recent papers [A. S. Merriweather, J. Sound Vibration 10, 369-379 (1971); W. Thompson, Jr., J. Sound Vibration 17, 221-233 (1961); and C. J. Boukwamp, J. Sound Vibration 17, 499-508 (1971)]. In contrast to the earlier papers, a time domain approach is presented in this paper to evaluate the radiation impedance. Rather than directly evaluate the well-known Kirchoff double surface integral, the impulse response or inverse Fourier transform of the impedance is first evaluated from the integral. A closed-form expression for the impulse response which is valid for all ring sizes is presented, and several interesting characteristics of the solution are noted and discussed. Since the impulse response and radiation impedance of a ring are a Fourier transform pair, the Fourier transform of the impulse response must be evaluated to obtain the impedance. Although the Fourier transform can not be readily evaluated analytically, an approximate expression for the radiation impedance of the ring can be obtained and is presented. Numerical results for the radiation 
impedance of different sized rings are also presented as a function of normalized frequency.

RR8. Sound Pulses Radiated in Air by Creeping Waves on Rigid Spheres and Cylinders. Richard H. Vocit, Naval Research Laboratory, IVashington, D. C. 20390.-The diffraction of sound pulses by metal spheres and cylinders in air was observed under conditions where the radiation from creeping waves could be compared with the specularly reflected pulses. The amplitudes of the radiated and reflected pulses were compared for various angles of scattering and for a range of frequency parameter $(k a)$ from 8 to 20 . The results were in agreement with theory in the case where the calculations were available. The attenuation of the creeping waves was determined from the decrease in amplitude of the sound pulses radiated tangentially as the creeping waves traversed increasing arc lengths along the surface of the body. In the case of pulses derived from truncated sinusoids two or three cycles in duration, the attenuation agreed with the steady-state theory in the observed range of (requency parameter from 8 to 40 .

RR9. Acoustic Radiation from a Point-Excited Infinite Elastic Plate. Alan D. Stuant, Ordnance Research Laboratory, The Pennsylvania State University, University Park, Pennsylvania 16802. - The acoustic pressure radiated by an infinite elastic plate excited by a dynamic point force is investigated. The effects of shear and rotary inertia in the plate have been in. cluded to ensure the proper representation of the high-frequency components of the radiated pressure. The pressure radiated by this coupled plate-fluid system is obtained with the use of integral transform methods. The inverse integral representation of the pressure field is evaluated by the asymptotic method of steepest descent, from which the near- and farfield pressure is determined directly. The farfield is associated with the saddle-point contribution, while the nearfield is associated with the poles of the integrand, on either Riemann sheet, that are enclosed by the path of steepest descent. A system of normalized variables is introduced that allows the radiated pressure to be expressed explicitly in terms of the material properties of the plate and the acoustic fluid, while being independent of the plate thickness. The frequency spectrum and directivity pattern of the farfield radiated pressure are obtained for a steel plate in air and in water. At frequencies above coincidence, two distinct peaks in the radiated pressure occur. The first peak to occur commences parallel to the plate; the second peak commences at the normal to the plate; both peaks reach a maximum angle as frequency is increased.

RR10. Transient Radiation from a Point-Ercited Infinite Elastic Plate. Alan D. StuakT, Ordnance Research Laboratory, The Pennsylvania Stale University, University Park, Pennsyluania 16802.-The acoustic pressure radiated by an infinite elastic plate excited by an impulsive point force has been determined. The effects of shear and rotary inertia in the plate have been included to ensure the proper high-frequency response. The calculation for the steady-state case has shown two distinct peaks or lobes in the farfield radiation pattern. The inverse two-sided Laplace transform reveals that the nature of the transient pressure waves are dependent on the point of observation relative to the three regions separating the two steady-state peaks. Precursor waves that arrive before the retarded time of the fluid medium were found to exist only in two of these regions, while the region about the normal to the plate contained only causal waves.
RR11. Acoustic Transmission Properties of Submerged Finite Cylindrical Shells. David S. PALletr, Ordnance Research Laboratory, The Pennsylvania Stale University, University Park, Pennsylvania 16802.-Statistical energy analysis procedures have been successfully employed for prediction of acoustic transmission properties of finite cylindrical shells in air in the work of White and of Manning and his associates. Extensive studies of Crocker and Price and their associates have dealt with acoustic transmission properties of plane panels in air. The present study directs attention to essential adaptations to these analytic methods that are necessary to account for fluid loading effects due to the presence of a dense fluid surrounding the exterior of a finite air-filled cylindrical shell. Attention is directed to the relative importance of the resonant and nonresonant acoustic transmission paths. The resonant response and transmission path is based upon resonant modal response properties, whereas nonresonant transmission is estimated upon the basis of transmission properties characterizing an infinite plane interface between two acoustic media. The nonresonant transmission path appears to be dominant for the uniform shells included in this study. Experimental measurements of the acoustic transmission properties of two uniform cylindrical shells have indicated good agreement between analytic and experimental data.

RR12. Pressure Waves in a Fluid Layer. J. R. EASter and P. J. Tonvik, Department of Mechanics, Air Force Institule of Technology, Wright-Patterson Air Force Base, Ohio 45433.The pressure field in a layer of compressible inviscid fluid between two parallel elastic plates is considered. Each plate is described by the Timoshenko-Mindlin plate equation, and a point time-harmonic force is assumed to be applied normally to one plate. The method of solution parallels that previously applied to a semiinfinite fluid bounded by one plate [Feit, J. Acoust. Soc. Amer. 40, 1489-1494 (1966)]. The Hankel transform was applied to the plate equations and the wave equation for the fluid, with the pressure and normal velocity at the interfaces being equated. The resulting inverse Hankel transform was evaluated both by numerical integration and by an approximation using the method of steepest descent. Separate expressions were developed in each case for results near the axis of symmetry and for large distances from that axis. Results for $10-\mu$ sec pulses were also obtained by the superposition of steady-state results for 20 frequencies. Satisfactory agreement between the two methods was obtained.

RR13. Vibration of a Seminfinite Fluid-Loaded Membrane. Huw G. Davies, Acoustics and Vibration Laboratory, Department of Mechanical Engineering. Massachusetts Instilute of Technology, Cambridge, Massachusetts 02139.--The vibration of and the acoustic radiation from a semiinfinite taut membrane is considered. The plane of the membrane is extended by a seiminfinite rigid baffle. A compressible fluid fills the halfspace on one side of the membrane and baffle. Two types of vibration are considered: (i) natural motion of the membrane due to an incoming acoustically slow wave on the membrane incident normally on the edge, and (ii) forced motion of the membrane, the force having sinusoidal space and time dependence. The forced motion thus is somewhat analogous to the single-mode-vibration of a finite membrane. The shape of the membrane and the associated acoustic radiation are discussed and compared with the case when fluid loading is not present. 
Session SS. Psychological Acoustics V: Masking

\author{
Irwin POLLaCK, Chairman \\ Mental Health Research Institute, University of Michigan, \\ Ann Arbor, Michigan 48104
}

Contributed Papers (10 minutes)

SS1. Measurement of the Critical Masking Interval. M. J. Penner, 5103 S. Mill St., Tempe, Arizona 85281, AND E. Cudahy, Department of Psychology, A rizona State University, Tempe, Arizona 85281 . - The critical masking interval, the time period during which noise is effective in masking a brief click, was measured in two experiments. The first paradigm was the temporal analog of Fletcher's critical band experiment. That is, the click was presented in the temporal center of the noise, and threshold was measured as the masker duration was decreased. The second paradigm investigated the temporal analog of Greenwood's [J. Acoust. Soc. Amer. 33, 484-502 (1961)] masking experiment wherein a click was moved through the noise burst. The two paradigms yielded similar numerical estimates of the critical masking interval (about $20 \mathrm{msec}$ ), although the critical masking interval was not affected by the stimulus' bandwidth.

SS2. The Effects of Masker Bandwidth on the Function Relating Detectability to Signal Duration. TerRence R. DOLAN, Parmly Hearing Institute and Psychology Department, Loyola University of Chicago, Chicago, Illinois 60026, AND DONALD E. RoBinson, Psychology Department, Indiana University, Bloomington, Indiana 47401.-The function relating detectability $\left(d^{\prime}\right)$ to signal duration for sinusoidal signals in Gaussian noise has been well documented. In general, it has been shown that $d^{\prime}$ is at a maximum when the signal duration is between 100 and 300 msec, and decreases for both longer and shorter durations. The present experiment replicated these results for both wide-band $(2900 \mathrm{~Hz})$ and narrow-band $(70 \mathrm{~Hz})$, continuous maskers. However, for pulsed signal and masker, the decrease in $d^{\prime}$ at short signal durations was obtained only when the noise masker employed was wide band. If a narrow-band noise was used as the masker, detectability continued to increase as signal duration was decreased.

SS3. Monaural Unmasking. Frederic L. Wightman and Tаммо Houtgast, Institute for Perception-TNO, Soesterberg, Netherlands._A classical forward-masking paradigm was used to measure the masked threshold of a brief $1000-\mathrm{Hz}$ signal presented shortly after the termination of a long, $1000-\mathrm{Hz}$ tonal masker. To our surprise, when a wide-band noise was added to the tone masker, at a level high enough that the tone masker was barely audible, the threshold for the signal decreased, by as much as $10 \mathrm{~dB}$ for some subjects. In other words, detection performance was actually improved by the addition of the ratlier intense noise masker; the noise somehow reduced the effectiveness of the tone as a masker. We suggest that this result reflects the operation of some kind of lateral-inhibitory process in the auditory system. The neural activity produced by the tone masker is thought to be suppressed, or inhibited by the addition of the noise (in much the same way as the neural activity produced by one tone has been shown to be suppressed by the addition of a second tone), thus reducing the masking effectiveness of the tone. In order for this "inhibitory" effect (and others recently reported in the literature) to be revealed, however, it is essential that the masker and signal not be presented simultaneously. In our experiment, with simultaneous presentation, adding noise never resulted in an improvement in performance. [Research supported in part by the Institute for Perception-TNO, Netherlands, and by a National Science Foundation Postdoctoral Fellowship awarded to the first author.]

SS4. Detection of Deletion and Insertion of Tones in Bandlimited Noise. W. T. Bourbon and V. L. LEmBo, Department of Psychology, Stephen F. Austin State University, Nacogdoches, Texas 75961.-The detectability of insertion (TI) and deletion (TD) of tones in a noise background was compared. Tones of $500 \mathrm{~Hz}$ were gated on or off for $100 \mathrm{msec}$ in noise with a spectrum level of $45 \mathrm{~dB}$ SPL. Psychometric functions were determined for two observers in a 2IFC task under wideband and bandlimited conditions of the masking noise. Under all conditions, the following results were obtained: (1) TI is more detectable than TD by $3 \mathrm{~dB}$ or more; (2) the slope of $P_{\text {(e) }}$ vs $E / N_{0}$ is greater for TD than for TI; and (3) the release from masking obtained with bandlimiting of the noise masker is greater for TD than for TI. The relationships of the present data to those of earlier studies and to critical band data are discussed.

SS5. Comparison of Direct and Indirect Measures of Critical Bands of the Monaural Chinchilla. W'. H. Seaton and C. TrahIOTIS, University of Illinois, Champaign, Illinois 61820.Typically, it is found that lower forms (rats, cats, and chinchillas) require a greater $\mathrm{S} / \mathrm{N}$ ratio than does man in order to detect the presence of a pure-tone signal in a broad-band noise. Assuming Fletcher's formula to be an adequate means of estimating the operating bandwidth of the auditory system, one could come to the conclusion that these lower forms have larger critical ratios, larger critical bands, and, therefore, poorer frequency resolving ability than does man. However, the behavioral index of auditory sensitivity utilized in these experiments does not permit separation of behavioral criterion and auditory sensitivity. It seems probable that lower forms are trained to maintain a "high" criterion (low false-alarm rate) and, thereby, produce threshold measures of relatively high $\mathrm{S} / \mathrm{N}$ ratio. Our efforts have been directed toward measuring both critical ratios and critical bands (band narrowing paradigm) utilizing both traditional threshold measures and criterion free indices of signal detection theory. Data obtained at signal frequencies of $500 \mathrm{~Hz}, 1,2$, and $4 \mathrm{kHz}$ will be discussed in terms of (1) the relationship of critical ratios to critical bands as a function of frequency and (2) the effect of criterion contamination on the estimate of the critical ratio and therefore on the estimate of the frequency resolving power of the organism.

SS6. Some Possible Attentional Aspects of Post-Stimulus Signal Processing. Richard E. Pastore, Department of Psychology, State University of New York at Binghamton, Binghamton, Nere York 13901.- Some possible attentional aspects of post-stimulus signal processing were investigated in a modified masked-signal detection paradigm. The signal was a $1000-\mathrm{H}_{\mathrm{Z}}$ sinusoidal waveform $20 \mathrm{msec}$ in duration and gated at 


\section{TH MEETING A ACOUSTCAL SOCIETY OF AMERICA}

positive zero-crossing of the waveform. The signal was masked by a broad-band $(360-3360 \mathrm{~Hz}$ ) Gaussian noise. The effect of a decrease in the amplitude of the masking noise, when confined to a limited frequency range, was investigated in terms of both the frequency range of the change and the temporal relationship between the offset of the signal observation interval and the onset of the decrease. An immediate decrease in masking noise in frequency regions outside the critical band of the signal decreased detectability.

SS7. Frequency Discrimination and Detection of Homophasic and Antiphasic Tonal Signals in Noise. Donald E. RoBINsoN, Psychology Department, Indiana University, Bloomington, Indiana 47401, AND TERrence R. DOLAN, Parmly Hearing Institute and Psychology Department, Loyola University of Chicago, Chicago, Illinois 60626.-Psychometric functions generated in a $2 \mathrm{ATFC}$ detection paradigm were compared to psychometric functions generated in a 2ATFC frequency-discrimination paradigm (see Henning, J. Acoust. Soc. Amer. 41, 4 (1967) ]. For frequency discrimination, two tones were presented, one in each of the two observation intervals. The observer's task was to indicate in which interval the signal of higher frequency occurred. With a frequency difference of $\mathbf{1 5}$ $\mathrm{Hz}$, the psychometric function for frequency discrimination of lomophasic signals was parallel to and slightly displaced to the right of the psychometric function for detection of homophasic signals. The psychometric function for frequency discrimination of antiphasic signals, however, had a significantly lower slope than the psychometric function for detection of antiphasic signals. Performance for frequency discrimination of antiphasic signals was similar to performance for detection of antiphasic signals at low $\mathrm{S} / \mathrm{N}$ ratios and similar to detection of homophasic signals at high $\mathrm{S} / \mathrm{N}$ ratios. The data are discussed in view of current models of binaural signal processing.

SS8. Pitch Shift and Masking by Sinusoids and Narrow-Band Noise. M. M. TAyLOR And S. M. Smiтh, Box 2000, Downsview, Ontario, Canada.-Direct masking of a sinusoid by a $10-\mathrm{Hz}$ noise band defines an apparent filter with a 3-dB bandwidth of as little as $7 \mathrm{~Hz}$ at $500 \mathrm{~Hz}$. This extreme sharpness is probably found because the center frequency of the filter shifts away from the noise frequency. We have found perceived pitch shifts of as much as $10 \mathrm{~Hz}$ for tones $30 \mathrm{~dB}$ above their masked threshold. With a simusoidal masker, less masking is lound for small frequency separations, presumably because beats between signal and masker are heard, whereas with the narrowband masker, they are masked by "beats" internal to the noise. When either a $10-\mathrm{Hz}$ noise band or a sinusoid fixed at $470 \mathrm{~Hz}$ is used to mask a $500-\mathrm{Hz}$ sinusoid, and a sinusoid at another frequency is used as a secondary masker, masking is enhanced by as much as $15 \mathrm{~dB}$, presumably because of extra beats generated within the critical band filter. Preliminary results suggest that the filter is over $200 \mathrm{~Hz}$ wide at $500 \mathrm{~Hz}$. Masking of beats by other beats could be a powerful tool in investigating the primary organization of the auditory system.

SS9. Masking with Ripple Noise and Spatial Frequency Analysis. W. A. Yost and B. Bergert, Communication Sciences Laboratory, University of Florida, Gaineswille, Florida 32601.-A low-pass noise $(1500-\mathrm{Hz}$ cutoff) with a sinusoidal variation in its amplitude spectrum (ripple noise) was used to mask a $1000-\mathrm{H}_{\mathrm{z}}$ signal (10 msec) in both a forward and a simultaneous masking procedure. The masked thresholds of the signal were measured as a function of the number of peaks (ripples) in the amplitude spectrum of the noise. The procedure was similar to that used by Houtgast who reported it at the 83rd meeting of the Acoustical Society. Houtgast used a pulsation threshold technique with ripple noise to study frequency selectivity. Pulsation threshold is similar to simultaneous forward and backward masking. The data of the present study indicate that results similar, but not identical, to Houtgast's can be obtained with forward masking alone. The frequency selectivity obtained with the forward masking procedure is sharper than has been previously obtained and might indicate the presence of lateral inhibition.

SS10. Masking of Pure Tones by Frequency Modulated Tones. I. M. Young and C. H. WenNER, Department of Otolaryngology, Jefferson Medical College, Thomas Jefferson University, Philadelphia, Pennsyluania 19107.-Pure-tone threshold measurements were made in the presence of frequency modulated tones in subjects with normal hearing. For a given masking intensity, effects at the carrier frequency of the FM tones, were found to be similar for a different frequency deviations and modulation rates. As masking intensity increased, effects at the carrier frequency increased linearly. Frequencies above and below the carrier frequency demonstrated a spread of masking being greater above the carrier frequency. This effect increased considerably for intensities above $60 \mathrm{~dB}$ re 0.0002 dyn $/ \mathrm{cm}^{2}$. As the modulation increased for a given frequency deviation, masking effects at frequencies above the carrier frequency behaved similarly to pure-tone masking. Upon increasing the frequency deviation for a given modulation rate, masking effects produced at frequencies higher than the carrier frequency were comparable to narrow-band noise. In comparing the masking at the carrier (center) frequency, the most effective method was narrow-band noise, then the FM tone, and the least effective method was pure tone.

SS11. Some Factors Affecting the Perception of Auditory Patterns. C. S. Watson, C. A. Benbassat, and IV. J. Kelly, Central Institute for the Deaf, St. Louis, Missorui, and l'ashington University, St. Louis, Missouri 63110.-Auditory patterns, each composed of a sequence of 10 tones 40 -msec long with frequencies between 250 and $900 \mathrm{H}_{z}$, were played in pairs. The patterns within each pair were either the same or differed by a $\Delta f$ added to one of the 10 tonal components. Just detectable values of this $\Delta f$ are quite small when the same patterns are used on every trial and when listeners know which tonal component may be changed [Watson and Wroton, J. Acoust. Soc. Amer. 51, 113(A) (1972)]. Presenting a new pattern on each trial was found to make the task significantly more difficult; however, the increase in difficulty was uniform whether one, two, four, or eight tonal components comprised the catalog from which the one to be varied was selected. Decay of the "auditory image," studied by varying the interval between standard and comparison patterns, was found to proceed at a rate similar to that for single tones.

SS12. Detection of Alterations of One Component in a Multiple Tone Complex. James H. Patterson, Jk., U. S. Army Medical Research Laboratory, Ft. Knox, Kentucky 40121.Experiments were conducted to determine the relative detectability of an increment, a decrement, and a phase shift of one component of a harmonic series of sinusoidal components. Data will be presented showing the detectability of these three types of alterations of one component as a function of the frequency of the component being altered. The results are interpreted in terms of the time-frequency resolving power of the auditory system.

SS13. Two-Tone Unmasking. B. Leshowitz and E. CUdahy, Department of Psychology, Arizona State University, Tempe, Arizona 85281 . - The detection of a brief tonal signal in the presence of a two-tone masker was investigated. The composite masker consisted of a gated sinusoid of constant amplitude (80 dB) added in quadrature with a continuous sinusoid. The frequency of the masker was $200 \mathrm{~Hz}$ higher than that of the signal. As the level of the continuous masker was increased from 60 to $85 \mathrm{~dB}$, signal threshold actually decreased by $15 \mathrm{~dB}$. 
This "unmasking" effect is interesting because simple stimulus-oriented models of detection cannot account for the data. Two-tone unmasking may be related to saturation effects reported for single units in the cochlear nucleus [R. L. Smith, J. Acoust. Soc. Amer. 52, 117 (1971)]. [This research was supported by an NIH grant.]

SS14. Regulation of Voice Amplitude by the Monkey (Macaca). J. M. Sinnotr, W. C. Stebrins, And D. B. Moody, Kresge Hearing Research Institule and Depariments of Olorhinolarygology, Psychology and Psycholinguistics, University of Michigan, Ann Arbor, Michigan 48104.--Increasing voice amplitude in the presence of noise has been referred to as the "Lombard reflex." Lane [J. Speech Hearing Res. 14, 677-709 (1971)] believes that the response is not reflexive, but that speakers learn to take their audience into consideration by raising their voices in noise. In this experiment, we determined the effect of noise on monkey vocalizations, which have long been considered to be primarily affective in nature and controlled by subcortical limbic structures [Robinson, Physiol. Behavior 2, 345-354 (1967)]. Monkeys were trained by operant conditioning procedures to vocalize for food reinforcement. Our results showed that the monkeys increased their voice level under a masking noise band of $200-500 \mathrm{~Hz}$ (fundamental frequencies for these monkeys ranged between 250 and $400 \mathrm{~Hz}$. The slope of the function relating vocal intensity to external noise level was approximately 0.2 , comparable to our own data from humans. The monkeys' voice amplitude was not noticeably affected by a high-frequency nonmasking noise band of $8-16 \mathrm{kHz}$. Thus both humans and monkeys appear to have a similar self-regulatory system of voice control in masking noise, despite alleged differences in the neural basis of human speech and monkey calls. [Research supported by grants from NIHI and Sigma Xi.]

Fridax, 1 December 1972

Silver Chimes East, 2:00 P.M.

\title{
Session TT. Speech Communication VIII : Temporal and Stress Factors in Speech
}

\author{
Stephen Krashen, Chairman
}

University of California, Los Angeles, Santa Monica, California 90405

\section{Contributed Papers (7 minutes)}

TT1. Central versus Peripheral Control of Vowel Duration. Iovanna Condax, Department of Linguistics, Princeton University, Neve Jersey 08540, AND RoBert Krones, Phonology Laboralory, Department of Linguistics, University of California, Berkeley, California 94720.-Although in English the vowel $[\mathrm{I}]$ is shorter than $[\mathrm{i}:],[0]$, and $[\mathrm{x}]$, only the $[\mathrm{I}] /[\mathrm{i}:]$ difference is traditionally recognized as linguistically significant, i.e., something the speaker "knows" and has control over. The differences between $[I] /[0, \mathfrak{x}]$, however, are usually attributed to constraints of the speech production mechanism. We tested this description by observing the effect on vowel durations of having speech produced without the normal speech organs, namely by having several subjects "speak" [sIs] "sis", [si:s] "cease", [sos] "sauce," and [sas] "sass," using a finger-operated switch that controlled a speech synthesizer. Although in normal speech, the subjects produced all four vowels with statistically significant duration differences, in the order $[\mathrm{I}<\mathrm{i}:<0<x]$, when "speaking" with the synthesizer only [I] was significantly shorter than the rest. We interpret these results as (1) supporting the traditional claim that the [I/i:] difference is part of the English speaker's linguistic competence, but (2) countering the claim that the $[I] /[0, \infty]$ difference is determined by the constraints of the speaking mechanisms and is not part of the speaker's linguistic competence. [Supported by the National Science Foundation and the Office of Naval Research.]

TT2. Fricatives-Their Physical Properties and Allophones. N. Umeda, R. B. Monsen, and M. Molter, Bell Laboralories, Murray Hill, New Jersey 07974.-Voiceless fricatives in American English were studied from readings of a continuous text. The wide range of duration ( $30 \mathrm{msec}$ to more than 200 msec) is explainable in terms of phonological conditions. For example, a fricative in the stressed-syllable initial position is about $20 \%$ longer than in the same phoneme sequence in unstressed position; single fricatives are longer than those in clusters with the same stress and positional conditions; a fricative preceded by a nasal is about $30 \%$ shorter than one under equivalent conditions with a nonnasal. Overall intensity of fricatives is not simply proportional to duration, but rather depends on stress and boundary conditions. Fricatives in prepausal position are longest but weakest. For a given phonological condition (e.g., word-initial stressed, and single), overall intensity reflects the sentence stress. There are consistent relationships between fricative intensity and spectral distribution. Recently we have argued that the stop consonants vary systematically to mark, among other things, word boundaries and the beginnings of stressed syllables. This study shows that allophonic variations of the fricatives carry the same information.

TT3. Epenthetic Stops between [1] and Following Sibilants. Hector R. Javkin and John J. Oilala, Phonology Laboratory, Department of Linguistics, University of California, Berkeley, California 94720.-An interesting phenomenon in phonology, one that has played a significant role in sound change, is the appearance of epenthetic stops between $/ 1 /$ and $/ s /$ in words such as "false" and "pulse," i.e., /folts/,/p^lts/. This occurs because of the failure to release the $/ 1 /$ contact before making the /s/ articulation. But such epenthetic stops are much less likely to occur when there is a $/ z$ / following the $/ 1 /$. We considered three possible explanations for this: (1) $/ 2$ / has a less complete area of closure than /s/ so the combined areas of closure for $/ 1 /$ and $/ z /$ do not form a complete stop; (2) complete closure can occur in both situations, but the oral air pressure buildup is smaller with $/ z$ / so that the stop is less perceptible, (3) differences in the timing of the tongue gestures for $/ \mathrm{s} /$ and $/ z /$. Preliminary investigations with one subject using a dynamic palatograph with computer display plus sampling of intraoral air pressure, suggested the probable correctness of (2). [Research supported by National Science Foundation and University of California President's Undergraduate Fellowship.]

TT4. Voiceless Consonant Durations in VCV Utterances. Jaemin Kim and Peter F. MacNeilage, Department of Linguistics, The University of Texas, Austin, Texas 78712.Durations of the eight English voiceless obstruents, $/ \mathrm{p} /, / \mathrm{t} /$, $/ \mathrm{k} /, / \mathrm{f} /, / \theta /, / \mathrm{s} /, / \mathrm{s} /, / \mathrm{c} /$ were measured from digitized oscil- 
lographic displays. Eight subjects produced each consonant 10 times in each of the four contexts $i-i, i-a, a-i$, and $a-a$. They read from randomly ordered lists and were instructed to place equal stress on both the surrounding vowels. The affricate $/ \breve{c} /$ had the longest total duration (closure plus "burst") and the fricatives $/ \mathrm{s} /$ and $/ \breve{s} /$ the next longest total duration Ior all subjects in all contexts. There were no consistent differences in total duration of the remaining five consonants. Closure durations for stop consonants were, in decreasing order, $/ \mathrm{p} /, / \mathrm{t} /, / \mathrm{k} /$, and burst durations showed the opposite order, results thus tending towards equal total stop consonant durations. Durational effects of the preceding vowel were not consistent across subjects. Low vowels were preceded by shorter total consonant durations than high vowels. The shorter durations were mainly observed during the burst of stops and affricates, and were least obvious in labials and interdentals.

TT5. Durational Characteristics of Word-Initial Consonant Clusters in English. DENNIS H. KLATT, Research Laboratory of Electronics and Department of Electrical Engineering, Massachusetts Institute of Technology, Cambridge, Massachusetts 02139.-A list of 224 English words beginning with prestressed consonant clusters or single consonants were recorded in the frame "Say . . . instead" by three talkers. Consonantal durations were measured from spectrographic analysis of the data. The results indicate that consonants in clusters are generally shorter than in a singleton environment, but that the amount of shortening is dependent on certain features of adjacent consonants. A system of durational adjustment rules is proposed in order to account for the statistically significant durational differences. If the consonantal duration in the singleton environment is considered as the standard duration, then rules (1) shorten two-element clusters by about $12 \%$; (2) shorten three-element clusters by about $18 \%$, (3) produce additional shortening in a consonant that is followed by a balistic closure; (4) produce additional shortening in a sequence of two voiceless consonants; (5) prevent labials from being shortened as much and shorten adjacent segments somewhat more to compensate; (6) lengthen a consonant if a preceding plosive causes aspiration to be present during a portion of the consonant; and (7) produces additional shortening in a sequence of two dentals. Implications for articulatory modeling, speech perception, and speech synthesis by rule are discussed. [Work supported in part by National Inst. of Health and by Office of Naval Research.]

TT6. Temporal Interactions within Phrase and Sentence Conterts. T. W. WRiGHT, Speech Research Laboralory, Temple Universily, Philadelphia, Pennsylvania 19122.--Recent timing control research has shown that when a smaller linguistic segment within a repeated utterance is produced either shorter or longer than its average duration, the resulting deviation constitutes a timing error that is temporally compensated by the remaining segments in the utterance. In the present study, timing errors and the extent to which they are compensated define different levels of temporal interaction. Temporal interactions were studied within a phrase spoken in isolation and within the same phrase spoken in a sentence context. The data, presented as significant negative Pearson correlations $(p<0.05)$ between various adjacent segment durations, support several tentative conclusions on the timing control of repeated utterances: (1) different-sized utterances have different levels of temporal interaction; (2) temporal interaction effects extend across phoneme, syllable, word, and phrase boundaries; (3) there appears to be no basic unit of timing for English utterances, but a hierarchy of units ranging in size from a $\mathrm{CV}$ or VC syllable to a sentence; and (4) identical durational errors may be compensated for by a variety of adjacent segment lengths.
TT7. Some Temporal and Autonomic Correlates of Sentence Production. Barbara G. Melamed and Robert J. Jakvella, Department of Psychology, Case Western Reserve Liniversity, Cleveland, Ohio 44106.-Sentences were obtained containing verbs that reler to cognitive states or processes and are grammatically capable of taking sentence complements (e.g., "know," "think," "amaze," and "delight"). The sentences were elicited in sentence production tasks by giving the verbs as lexical content or asking questions containing them. Some control conditions were also employed, including subject reading of some of the sentences aloud. Concurrent measures of speaking onset and duration, heart-rate variability, and galvanic skin response were taken during production of the sentences. Grammatical properties of the sentences are described and related to these temporal and autonomic variables.

TT8. Silent Interval and Vowel Duration Effects on Pause Magnitude Estimation in Intrapause and Interpause Environments. JoHn A. RUPF, Department of Electrical Engineering, Universily of Kansas, Lawrence, Kansas 66044, AND KENNETH F. Ruder, Bureau of Child Research, University of Kansas, Lawrence, Kansas 66044.-The stimuli for this experiment were derived from two sentences, each containing the words free criminals. In one sentence these two words occurred within a phrase, and in the other sentence a phrase boundary occurred between the words. The vowel duration in free and the silent interval preceeding criminals were systematically and independently varied. Vowel durations of $100,200,300,400,500$, and 600 msec were paired with silent intervals of $0,50,100$, 150,250 , and 500 msec for both sentences. Subjects were asked to make direct magnitude estimates of the perceived pause between free and criminals in each of the stimulus sentences. Results indicated that given durations of vowel and silent interval result in similar perceived pauses in intraphrase position than in interphrase position. Power law scaling exponents were larger for vowel duration than for silent interval in all conditions. [This research supported by the National Institutes of Health.]

TT9. More Temporally Segmented Speech. A. W. F. HUGGINS, Research Laboratory of Electronics, Massachuselts Institute of Technology, Cambridge, Massachuselts 02139.-The intelligibility of alternated spech passes through a ninimum at an alternation rate of about $3 \mathrm{~Hz}$. The critical parameter defining the minimum is not the duration of the bursts of speech reaching the listener, but rather the amount of speech each burst contains [J. Acoust. Soc. Amer. 36, 1055 (1964)]. Recent work with temporally segmented speech [J. Acoust. Sor. Amer. 52, 176(A), (1972)] showed the decline and recovery of intelligibility, as switching rate is increased, to be due to different effects. The decline depends on the decreasing duration of the speech bursts, but the recovery depends on the decreasing duration of the silent intervals. Since the mininum is determined by both these effects, the amount of speech contained in the speech bursts cannot be the only critical parameter, as stated above. To resolve this conflict, an experiment was perlormed with temporally segmented speech. Intelligibility was measured as a function of silent interval duration, speech interval duration, and speech interval content, the latter two being varied independently by varying the playback speed of the speech. [This research was supported by NIH grant.]

TT10. Temporal Variability of Repeated Utterances by Normal Speakers and Stutterers. George Allen and Margaret COOPER, University of North Caralina, Chapel Hill, North Caralina 27514.-Ten normal speakers, five stutterers, and five "cured stutterers" each repeated four different speech 
samples several times. The four samples were (1) an "easy" sentence, (2) a "hard" sentence, (3) an "easy" paragraph, and (4) a "hard" paragraph, where "easy" and "hard" refer to high and low frequency of occurrence in English of the content words used. In a separate fifth task, each subject tapped his finger as steadily as he could for $4 \mathrm{~min}$. From each of these five repeated behaviors will be derived a variability measure (relative variance, or amount of variance per unit of time) that describes each subject's accuracy in repeating the time program of that behavior. The reliability of this measure, i.e., the stability of the measure across tasks for each subject will be examined, and its validity will be related to the normal and stuttering populations tested.

TT11. Speaking Rate and the Delivery of Phonetic Commands in Standard American English. William L. ABler, Stanford Medical Center, Stanford California 94305.-Measurements of the acoustic segments in the sentences, get your feet off the table, get your seal off the table, get your foot off the table, get your soot off the table, in which individual acoustic segments of different intrinsic durations were interchanged, show that these changes do not induce compensation or other temporal or acoustic consequences within the syllable. However addition of new acoustic segments into the two-stress sentences, Jóe died, Jósie died, Jóseph díed, Jósephine díed, does induce temporal compensation within the interstress interval. Thus the duration of the stressed $/ \%$ in these sentences contracted $45 \%$ from the first to the last sentence when all were spoken at a constant speaking rate. Further measurements of serial repetitions of individual sentences, e.g., Jósie died, Jósie díed, . . . show that the acoustic interval most stable in duration is the interstress interval when the sentences are spoken at a constant speaking rate. These findings support the view that the phonetic commands of Standard American English are delivered to the vocal muscles in groups spanning the interstress interval, and not spanning just the syllable.

TT12. Phonetic Disambiguation of Syntactic Ambiguity. ILSE LEHISTE, Department of Linguistics, The Ohio State University, Columbus, Ohio 43210 . - It has been frequently claimed that the meaning of syntactically ambiguous sentences (such as "Visiting relatives can be a nuisance") can be made explicit by phonetic means such as stress and intonation. This study describes some ways in which such disambiguation can be accomplished. Fifteen ambiguous sentences were first read by four speakers. The ambiguities were then pointed out, and each speaker stated which meaning he had intended to produce. Each sentence was then produced again twice, the speaker making a conscious effort to differentiate between the two potential meanings. Thirty listeners tried to identify the meaning which the speakers had intended. In 10 out of 15 cases, listeners performed at better than chance level, which implies that the intention of the speakers was successfully communicated to the listeners. The suprasegmental patterns employed by the speakers in successful disambiguation were established by acoustic analysis. While stress and intonation play a part, timing seems to have been the principal means by which the two meanings of the sentence were differentiated.

TT13. Acoustic Features of Disyllabic Stress Patterns in Speech and Singing. D. N. BENNETT, Department of Speech, University of Washington, Seattle, Washington 98195, AND T. T. Tavener, Department of Music, Whitworth College, Spokane, Washington 98415.-Trained male singers sang (chest and falsetto registers) and spoke $\mathrm{CVCV}$ syllables embedded in a carrier phrase, using as stressed-unstressed/unstressed-stressed paradigm. The consonant / $t$ / was employed throughout in combination with the vowels /i-a-u/. All syllables were segmented into three acoustic elements: (1) silent interval, (2) noise burst, and (3) phonation. The silent interval plus the noise element were considered to correspond with the consonant, and the phonation segment with the vowel. In appropriate instances, measures were made of duration, amplitude, fundamental frequency, and formant characteristics of the segments from spectrographic records. Relations among segments are reported as a function of modes of utterance and stress. Inferences are drawn concerning possible differential articulatory and phonatory demands on the production system as a consequence of syllabic stress during singing and speech.

TT14. Coarticulation and Speech Perception: The Effect of Stressing and Consonant Cluster. W. H. ManNinG, Division of Speech Pathology and Audiology, University of Nebraska, Lincoln, Nebraska 68508, aND D. S. BEASLEY, Departmeni of Audiology and Speech Sciences, Michigan Slate University, East Lansing, 48823.-Degree of coarticulation as defined in previous research [W. Manning, D. Beasley, and T. Beachy, I. Acoust. Soc. Amer. 52 (1972)] was investigated as a factor in the perception of human speech. Fifty subjects listened to 360 two-word combinations which provided 180 conditions of greater $/ \mathrm{sp} / \mathrm{h} / \mathrm{st} /$, and /sk/ coarticulation, and 180 conditions of lesser $/ \mathrm{sp} / \mathrm{h} / \mathrm{st} /$, and $/ \mathrm{sk} /$ coarticulation. Stimuli were presented in a stressed and nonstressed manner. Subjects were randomly divided into five groups of 10 individuals each and administered the stimuli in one of five $S / N$ ratios. The results reveal that mean percentage correct scores were significantly higher for the more coarticulated consonant clusters under the unstressed condition, while the less coarticulated consonant clusters were perceived more accurately under the stressed condition. Where differences in stressing could not be employed in the decision of $s$ placement, coarticulatory cues appeared to assume greater importance in the perceptual task.

Friday, 1 December 1972

El Dorado West, 2:00 P.M.

Session UU. Physical Acoustics VI : General

Mack A. Breazeale, Chairman

Department of Physics, University of Tennessee, Knoxville, Tennessee 37916

\section{Contributed Papers (12 minutes)}

UU1. An Interpretation of the "Frequency of Least Reflection" at the Rayleigh Angle for a Liquid-Solid Interface. Werner G. Neubauer, Naval Research Laboratory, Washington, D.C. 20390.-Ultrasonic receiver detection of a reflected beam at the Rayleigh angle has resulted in the interpretation of a minimum amplitude for a given material at some frequency called the frequency of least reflection. Recently it has been found that, in general, it is more meaningful to regard the reflection of a finite beam as energy redistribution rather than a reemergence of the beam in the 
same form as it had on incidence. At low megahertz frequencies, that redistribution may be regarded as consisting of threc distinct regions: a specular region, a Rayleigh wave radiation region, and a mutual interaction region of the specular and Rayleigh radiations. This model based on schlieren photographs is used to show that the "frequency of least reflection," observed with a hydrophone, can be explained in terms of energy redistribution.

UU2. Direct Measurement of Rayleigh Wave Velocity by Schlieren Visualization and Estimates of Shear Velocity Therefrom. Louis R. DragonetTe, Werner G. Neubauer, and Joseph A. Bucaro, Naval Research Laboratory, Washington, D.C. 20390.-Schlieren visualization experiments are used to obtain direct, accurate measurements of Rayleigh phase velocity on various materials. Illumination of a plane solid surface underwater at the Rayleigh angle produces an easily identifiable null strip in the radiation which uniquely defines the Rayleigh angle. 'This strip is caused by the mutual cancellation of equal amplitude specular and Rayleigh radiations, which are $180^{\circ}$ out of phase at the Rayleigh angle. Rayleigh velocities are calculated from the measured angle by a simple equation. The measured Rayleigh velocities are used to give estimates of shear velocity, and these estimates are compared with direct shear velocity measurements. Attenuation of the Rayleigh wave in the low megahertz region is given for six materials, and shown to agree with theoretical predictions.

UU3. Rayleigh Wave Study of Ionic Diffusion and Stress Buildup in Glass. J. A. BuCARO, Naval Research Laboratory, Washington, D.C. 20390, AND J. H. Simmons, National Bureau of Standards, Washington, D.C. 20234.- The velocity of elastic surface waves has been studied as a function of frequency on a system of ion exchanged glasses. The ion exchange was carried out by immersion in a high-temperature salt bath at several temperatures and for times ranging from $\frac{1}{3}$ to $259 \mathrm{~h}$. The results of this study allow the determination of the ionic diffusion coefficient and its activation energy. The mechanism of dispersion is discussed in terms of mass and volume changes and stress buildup. Since ion exchange is currently used to strengthen glass, the above technique also appears to be ideal for the nondestructive testing of glass strengths.

UU4. Critical Angle for Reflection from a Water-Quartz Interface. Edmund G. Henneke II and Gerald L. Jones, Department of Engineering Science and Mechanics, Virginia Polytechnic Institute and State University, Blacksburg, Virginia 24061, John Moyu Liu and Robert E. Green, Jr., Department of Mechanics and Materials Science, The Johns Hopkins University, Baltimore, Maryland 21218.-A new, general definition is given for the critical angle of reflection in anisotropic materials. While the critical angle for reflection at a liquid-isotropic-solid interface can be written in the form $\sin \theta_{\mathrm{c}}=v_{I} / v_{R}$, we show here that, in general, for an anisotropic solid, the right-hand side of this expression must be modified by the factor $\cos \phi / \cos \beta$, where $\phi$ is the angle between the normal to the refracted wave in the solid and its energy flux vector and $\beta$ is the angle between the energy flux vector and the component of the refracted wave normal on the interface. Numerical calculations have been made for this general definition for a water-quartz interface for planes of incidenre about the $z$ axis (the $x y$ plane being the interface). These calculations are compared with the value of the critical angle as determined by the unmodified definition. It is shown that this earlier definition can lead to large errors when one uses the experimental technique of measuring the critical angle to evaluate the elastic constants of the solid.
UU5. Experimental Determination of the Critical Angle for Reflection from a Water-Quartz Interface. JoHN MOYU LIU and Robert E. Green, JR., Department of Mechanics and Materials Science, The Johns Hopkins Universily, Baltimore, Maryland 21218; Edmund G. Hexneke II and Gerald L. JoNEs, Department of Engineering Science and Mechanics, Virginia Polytechnic Institule and State University, Blacksburg. Virginia 24061. - The critical angle of reflection for a wave incident from water upon the $x y$ plane of quartz has been measured for planes of incidence about the $z$ axis. These experimental measurements are compared with the theory that predicts that the critical angle for reflection from an interface of an anisotropic material occurs when the energy flux vector of the refracted wave becomes parallel to the interface. This differs from the usual definition of the critical angle as that angle for which the refracted wave normal beconkes parallel to the boundary. The new definition of critical angle modifies the usual expression $\sin \theta_{c}=V_{I} / V_{R}$ by the factor $\cos \phi / \cos \beta$ as discussed in the previous abstract.

\section{UU6. Abstract withdrawn.}

UU7. Temperature Rise in Biological Tissue during Focused Ultrasonic Irradiation: Some Theoretical Subtleties. AlLAN D. Piskce, Department of Mechanical Engineering, Massachusetts Institute of Technology, Cambridge, Massachusetts 02139. - During focused irradiation, the absorption of ultrasound causes a net increase of temperature in the focal region. A topic of some current interest is the extent to which the temperature history or the peak temperature reached correlates with the probability of lesion formation. Because of the practical difficulties associated with measurement of very rapid temperature rises, theoretical models are sometimes used to infer the temperature history during intense but short duration pulse irradiation. The existing models [Pond, J. Acoust. Soc. Amer. 47, 1607 (1970)] Robinson and Lele, [J. Acoust. Soc. Amer. 51, 1333 (1972)] neglect a number of effects that might warrant some study. Among these is the fact that, for a given acoustic power output, the intensity at the focus may tend to decrease with time because when the medium becomes heated by the wave, the medium tends to refract wave energy away from the higher temperature and, hence, higher sound speed regions. In the present paper, it is shown that the fractional decrease in intensity is of the order of $0.05(\Delta T)^{1}$, where $\Delta T$ is the temperature rise in degrees centigrade (typically, $30^{\circ} \mathrm{C}$ ). Possible implications of this and other effects in the reinterpretation of intensity versus damage threshold irradiations duration time data are discussed quantitatively.

UU8. Computerized Enhancement of Ultrasonic Echograms. C. R. MEYER AND D. L. CARLsON, Biomedical Engineering Program, Lowa Stale Unzversuy, Ames, lowa $50010 .-$ In the area of pulsed ultrasonics, there has been an effort to improve the resolution of compound scan ( $C$-scan) echograms. Most. of the effort has been directed toward reduction of the transducer's beamwidth with such developments as composite 
transducers or focused transducers with variable focal distances. Problems of image saturation and artifact generation have received relatively little attention. This study reports on a method of generating without image saturation, a $C$-scan echogram whose resolution is independent of the transducer's large beamwidth. Normal $C$-scan information is recorded on analog magnetic tape and temporally expanded for construction of the target's positional likelihood distribution using a digital computer. Targets possessing vertical symmetry in the region scanned by the ultrasonic beam are used in a water bath to investigate the system resolution. After the likelihood distribution is constructed, it may be plotted, or numerical methods can be used to plot the processed echogram. In constructing the processed echograns, only the positions of significant local maxima are plotted. A processed echogram of the whole region, or of any segment, can be plotted using any desired scale factor. The processed echograms are compared to echograms degraded by simulated image saturation and the improvement in resolution of the processed echograms is clearly shown. Artifacts due to beamwidth and multiple reflections are missing in the processed echograms.

UU9. A Survey of Sound Propagation in Soils. T. G. Winrer, Department of Physics, University of Tulsa, Tulsa, Oklahoma 74104.- One of the most attractive applications of acoustical holography is the location of objects and formations buried near the surface of the earth. But the first step is to learn the sound propagation characteristics of the earth. Dilitational wave velocities in unconsolidated soils range from 500 to $5000 \mathrm{ft} / \mathrm{sec}$ within the first $50 \mathrm{ft}$. Shear velocities are $30 \%-60 \%$ of dilitational velocities. The absorption coefficient is directly proportional to frequency and is typically $1-5 \mathrm{~dB} / \mathrm{ft}$ at 1000 Hz. The effect of amplitude, frequency, void ratio, pressure, and moisture content on absorption and velocity will be reported. But velocity and absorption coefficients are the least of the problem. The layered nature of the medium leads to multiple transmission paths and the variation of velocity with position in the medium leads to complex refraction effects.

Friday, 1 December 1972

El Dorado East, 2:00 P.M.

Session VV. Underwater Acoustics VI : Nonlinear Acoustics

\author{
Robert T. Beyer, Chairman \\ Department of Physics, Brown University, Providence, Rhode Island 02912
}

Contributed Papers (10 minutes)

VV1. Parametric Acoustic Arrays. C. C. Fox And O. L. Akervold, Honeywell, Marine Systems Cenler, Seattle, Washington 98107.-Parametric acoustic array performance has been demonstrated with several different transducers. Threeinch-diameter transducers with primary frequencies between 250 and $300 \mathrm{kHz}$ have produced good parametric acoustic beams at 10,20 , and $40 \mathrm{kHz}$ difference frequencies with nearly constant beamwidth across this frequency band. A parametric acoustic array operating at $20-\mathrm{kHz}$ difference frequency was compared to a $100-\mathrm{kH}_{\mathrm{z}}$ conventional transducer for bottom profiling capability in Lake Washington. Echograms were obtained demonstrating the capability of the lower frequency beam to produce subbottom, as well as bottom returns.

VV2. Parametric Echoscanner for Biomedical Diagnostics. T. G. Muir, C. M. Talkington, B. S. Shaw, R. S. Adalr, AND J. G. WILLETTE, Applied Research Laboratories, The University of Texas al Austin, Austin, Texas 78712.-The nonlinear interaction of two high-lrequency high-intcnsity waves to produce a highly directive difference frequency wave is known to be of value for systems requiring good angular resolution at operating frequencies not limited by absorption. The technique has recently been successiully applied to problems in marine bathymetry [J. Acoust. Soc. Amer. 50, 1085-1087 (1971)] and archeology [Paper F6, 83rd Meeting, Acoust. Soc. Amer.]. An analysis of the potential utilization of this technique in ultrasonic diagnostics is presented in the present paper. This analysis includes a comparison of parameters attainable by both nonlinear and conventional methods, as well as a design description of a prototype parametric echoscanner. This device utilizes a 0.25 -in. piston to transmit interacting waves at 7.25 and $8.75 \mathrm{MHz}$. The results of measurements on the amplitude and beampattern of the $1.5-\mathrm{MHz}$ difference-frequency wave are presented. Echolocation data obtained with several simple targets are also presented to demonstrate the resolution capability of the instrument.

VV3. Performance of an Acoustic Parametric Source. ANTHONy I. Eller, Naval Postgraduate School, Monterey, California 93940, and Naval Research Laboratory, Washington, D.C. 20390.-The performance of an acoustic parametric source was investigated at the NRL Acoustic Research Tank Facility. The source utilized a USRD type E8 transducer, driven simultaneously at two frequencies close to the resonance frequency of $1.5 \mathrm{MHz}$. Narrow-beam difference-frequency waves, at frequencies ranging from $30-200 \mathrm{kHz}$, were generated by the nonlinear nature of acoustic propagation. Measured values of the difference-frequency SPL and a sequence of radiation patterns illustrate beam formation in the nearficld of the parametric source. The results show that the E8 tranoducer is suitable for use as a parametric source and also demonstrate the fiexibility of the NRL facility as a research tool in nonlinear acoustics.

VV4. Mathematical Model for a Standing Wave Acoustic Parametric Source. A. L. Van Buren and Peter H. Rogers, Naval Research Laboralory, Washington, D.C. 20390.-A standing wave acoustic parametric source (SWAPS) has been proposed as a small, efficient, low-Irequency, nearly omnidirectional underwater sound source. SWAPS is envisioned to be a cylindrical tube driven on one end by a piston transducer and terminated on the other end by a thin plug of pressure release material to form a resonant cavity. When the piston is driven at two neighboring resonance frequencies, 


\section{TH MEETING - ACOUSTICAL SOCIETY OF AMERICA}

the resulting large amplitude primary sound waves mix nonlinearly to produce secondary sound waves. The secondary wave at the difference frequency is of low frequency' so that it passes easily through the relatively thin tube walls and radiates to the farfield. The farfield radiation at the difference frequency is calculated by use of the virtual source density method. The results are expressed in ternis of a quality factor which represents the resonance propertics of the cavity. Comparison is made to a traveling wave acoustic parametric source using the same piston transducer.

VV5. Parametric Detection of a Low-Frequency Plane Wave by a Circular Piston Beam. Peter H. Rogers, Naval Research Laboratory, W'ashington, D.C. 20390, AND A. O. Williams, Jk., and James M. Barker, Physies Department, Brown University, Providence, Rhode Island 02912.-Westervelt's prediction [I. Acoust. Soc. Amer. 35, 535-537 (1963)] that an acoustic signal of low frequency can be detected by a parametric endfire array-i.e., by nonlinear interaction with a "pump" beam of high frequency-is analyzed in the case of a plane wave of low frequency and a highly directional bean from a circular plane piston used as a pump. To first-order perturbation theory, at field point $(r, z)$, the $z$ component of particle velocity for the interaction component at the difference frequency is proportional to the product of the pistonbeam function at $(r, z)$ (but calculated at the difference frequency) and the response function of a truncated endfire array of length $z$ (calculated at the low frequency). The receiving directivity of the system is thus determined by the endfire response function alone, regardless of the piston's properties. The piston-beam function is, however, a factor in determining the system's receiving sensitivity. Work continues on generalizing the theorem to arbitrary directional pump transducers.

VV6. Investigation of the Parametric Radiator as a WideBand Calibration Source. W. L. KonRad and J. G. Navin, New London Laboratory, Naval Underwater Systems Center, New London, Connecticut 06320.-The parametric difference frequency radiator appears to offer advantages over conventional sources used for hydrophone calibration, i.e., wide bandwidth with a single transducer, narrow beamwidths, and short pulselengths. The combination of these characteristics promises sinplification of calibration procedures and elimination or reduction of boundary reflection problems. Experiments are described which measure the performance of the parametric source for calibration purposes and which point out some of the special problems inherent in this new technique.

VV7. A Closed Form Expression for the Source Level of a Finite Amplitude Parametric Array. James F. Bartram, Raytheon Company, Portsmouth, Rhode Island 02871.-In a Letter to the Editor [J. Acoust. Soc. Amer. 52, 1042 (L) (1972)], the author has derived a closed form expression for the on-axis farfield pressure of the secondary wave generated by a finite amplitude parametric array in which the primary waves are collimated plane waves. This formulation takes into account the effects of absorption and saturation due to shock formation. The present paper shows that this solution can be modified to account as well for spherical spreading and diffraction. It is even possible to treat nearfield buildup effects in a rather crude fashion. The thus modified expression remains in closed form, and is therefore useful for system engineering purposes.
VV8. A Computer Algorithm for Predicting Propagation of Intense Acoustic Signals of Arbitrary Waveform. F. M. Pestokius and I). 'T. Blackstock, Applied Research Laboratories, Liniversily of Texas, Austin, Texas 78712. - The planewave propagation law $d t / d x=\left[c_{0}+\beta u\right]^{-1} \doteq c_{0}{ }^{-1}\left[1-\beta u / c_{0}\right]$ is used as the basis of a computer algorithm to calculate the distortion of a finitc-amplitude wave. Here $x$ is distance, $t$ time, $u$ particle velocit $y$, and $c_{0}$ small-signal sound speed; for gases $\beta=(\gamma+1) / 2$, for licuids $\beta=1+B / 2 A$. Starting with a time waveform of arbitrary shape, we use a finite-difference form of the above law to calculate the wave shape at successive small distinces. After each step in distance the waveform is scanned for muluivaluedness to determine whether shocks have forned. When a shock is identitied, its propatgation is described thereaficr by the weak-shock relation $(d t / d x)_{a} \doteq c_{0}{ }^{-1}\left[1-\beta\left(u_{a}+u_{b}\right) / 2 c_{0}\right]$, where $u_{a}$ and $u_{b}$ are values of $u$ ahead of and behind the shock, respectively. In growing, decaying, and merging, the shocks ultimately determine the shape and amplitude of the wave. The algorithm has been tried on several initial signals, including $N$ waves, sinusoids, and raudomly generated waveforms. In general, the algorithm gives excellent agreement with known analytical results. Comparison has also been made with experimental data on pulsed sinusoidal waves in a tube. The algorithm has been developed as a tool to study propagation of very intense noise. [I'ork supported by ONR, AFOSR, and USN Postgraduate school.]

VV9. Finite-Amplitude Losses in Spherical Sound Waves. Harold M. Merklinger, Defence Research Establishment Allantic, Darlmouth, Nona Scotia, Robert H. Melden, New London Laboralory, Naval Lnderwater Systems Center, New London, Connecticut 06320, AND MaRK B. MOFfETT, University of Rhode Island, Kingston, Rhode Island 02881.-Using an approach suggested by Westervelt [3rd Int. Congr. Acoust., Stuttgart (1959)], the excess absorption in a plane, traveling sound wave due to nonlinear effects can be predicted with remarkably accuracy. The same approach is applied to spherically diverging sound waves, evaluated for a number of cases of practical importance in underwater acoustics, and compared with existing data.

VV10. Aspects of the Finite Amplitude Interaction of Multiple Tone Signals. Roger G. Pridham, Raytheon Company, Portsmouth, Rhode Island 02871 . - Two results are given that pertain to the effects of finite amplitude distortion on a multiple tone (i.e., multiple sinusoid source) signal. The first result is a sccond-order perturbation solution to plane-wave Burgers' equation for a mulciple-tone source with arbitrary amplitude and phasing. This solution is interpreted to predict the spectral distortion of the primary waves at moderate levels. The second result concerns the relationship of the shock distance for fixed source tone levels and frequencies to the relative phases of the source tones. It is shown that the shock distance is minimized when the source components are in phase. However, the shock distance maximization problem is shown to be equivalent to the classic peak factor problem, which has so far defied solution. While an exact maxinization is not possible, a procedure is given for phase selection that yields shock distances that are considerably greater than the minimum shock distance when many source tones are present. It is pointed out that shock distance maximization may significantly reduce distortion effects. This is particulary true if the shock can be made to occur in the farfield.

VV11. Transient Pressure Attenuation by Nonlinear, Resilient, Cylindrical Scatterers. Thomas L. GeErs, Lorkheed Palo Alto Research Laboratory, Palo Alto, California 94304.Temporal convolution techniques [T. L. Geers, J. Acoust. Soc. 
Amer. 51, 1640-1651 (1972)] are used to obtain solutions for the problem of transient acoustic scattering by nonlinear cylindrical scatterers. These scatterers behave like cavities during the initial stages of motion, but then offer increasing dilatational resistance with increasing deformation. In contrast to the results of previous studies [M. L. Baron, J. Appl. Mech. 24, 501-505 (1957) and J. Appl. Mech. 28, 135-136 (1961)], it is found that such "modified cavities" are indeed capable of effective transient pressure attenuation in the surrounding fluid without the generation of unacceptable pressure pulses at later times. Parameter studies for plane, step-exponential incident waves are performed to establish suitable ranges for the modified cavity stiff ness parameters.

VV12. Absorption of Sound by Sound. Peter J. Westervelt, Applied Research Laboratories, The University of Texas at Austin, Austin, Texas 78712, and Brown University, Providence, Rhode Island 02912.-The exact solution for first order nonlinear scattering of two plane waves of sound [J. Acoust. Soc. Amer. 29, 934 (1957)] showed the scattered density to be

$$
\rho_{s}=c_{0}^{-2} E_{12}+\frac{1}{2} \omega_{1}{ }^{-1} \omega_{2}^{-1}\left(\cos \theta+\frac{1}{2} \Lambda\right)\left[\sin ^{-2}\left(\frac{1}{2} \theta\right)\right] \nabla^{2} W_{12} \text {. }
$$

The singularity at $\theta=0$, which occurs when the primary waves are collinear, may be removed by applying to this solution the operator

$$
\left\{1-\exp \left[\left(\left|\frac{\partial}{\partial t}\right|\left|c_{0} \nabla\right|^{-1}-1\right)\right] \mathrm{r} \cdot \nabla\right\} .
$$

The resulting solution is now valid for $\theta=0$ in addition to all other intersection angles. This new solution has been applied with the conservation of energy [P. J. Westervelt, in L. Cremer, Ed., Proc. Int. Cong. Acoust., 3rd (Elsevier, Amsterdam, 1960), p. 316], to determine the attenuation of one plane wave with wave vector $k_{1}$, interacting with an isotropic distribution of waves having the energy density spectrum $u(k)$. The general result for the pressure attenuation coefficient is

$$
\begin{aligned}
\alpha=\left(2 \rho_{0} C_{0}^{2}\right)^{-1}\left(1+\frac{1}{2} \Lambda\right)^{2} \pi\left[\int_{0}^{k_{1}} k u(k) d k+2 k_{1}\right. & \int_{k_{1}}^{\alpha_{1}} u(k) d k \\
& \left.+k_{1}^{2} \int_{0}^{k_{1}} k^{-1} u(k) d k\right] .
\end{aligned}
$$

In the event $k_{1}$ is much less than any component $k$ of the interacting background, a great simplification results and

$$
\alpha=\left(2 p C_{0}\right)^{-1}\left(1+\frac{1}{2} \Lambda\right)^{2} \pi k_{1} E,
$$

in which $E$ is the energy density of the background radiation. This result is supported by experiments on the attenuation of sound in superfluid helium [H. J. Maris, Phys. Rev. Lett. 28, 277 (1972)]. [This work was supported by the U. S. Office of Naval Research.]

VV13. Laser Excited Broadside Acoustic Array. Peter J. Westervelt and Richard S. LaRson, Applied Research Laboratories, The University of Texas at Austin, Austin, Texas 78712, and Brown University, Providence, Rhode Island 02912. - A high-power modulated laser beam in a fluid acts like a broadside acoustic directional array at the modulation frequency. The laser is assumed to generate a beam having average power $P_{0}$ with $100 \%$ modulation at the frequency $\omega$. The radiated acoustic intensity $I$ at a distance $r$ far from this source is

$$
I=\frac{\left(\alpha \beta \omega P_{0}\right)^{2}}{32 \rho_{0} c_{0}\left(\pi r c_{p}\right)^{2}}\left[\frac{1}{\alpha^{2}+k^{2} \cos ^{2} \theta}\right] \text {, }
$$

in which $\alpha$ is the intensity attenuation coefficient for the laser beam, $\theta$ is the angle measured from the laser beam direction, $k=\omega / c_{0}, \beta$ is the coefficient of thermal expansion, $c_{p}$ is the specific heat per unit mass, $c_{0}$ is the speed of sound, and $\rho_{0}$ is the density. Maximum radiation occurs at $\theta=\pi / 2$, in a direction perpendicular to the laser beam. [This work was supported by the U.S. Office of Naval Research.]

VV14. Receiving Calibration and Beam Pattern Measurements of a Large Sonar Array with a Parametric Transmitting Array. J. J. 'Truchard and J. G. Willette, Applied Research Laboratories, The University of Texas al Austin, Austin, Texas 78712.-A classical problem encountered in the receiving calibration and beam pattern measurement of a large, conventional sonar array is the difficulty involved in producing a plane wavefront at the face of the large array when reflecting boundaries are present. In this paper, an attempt will be made to show that the parametric transmitting array can be used to minimize this problem. Two experimental beam patterns are presented for the case of an 8-ft aperture array receiving at $5 \mathrm{kHz}$. One beam pattern was taken by using an ornnidirectional $5-\mathrm{kHz}$ source. The other was taken by using a parametric array sound source having primary frequencies of 70 and $75 \mathrm{kHz}$-thus, a difference frequency of $5 \mathrm{kHz}$. Theoretical results are presented for the parametric array which demonstrate the uniformity of phase and amplitude across the 8-ft aperture. The feasibility of absolute receiving calibration with the parametric array is discussed.

\title{
Session XX. Flow and Other Noise
}

\author{
R. E. HAYDEN, Chairman \\ Bolt Beranek and Newman Inc., 50 Moulton Street, Cambridge, Massachusetts 02138
}

Invited Paper (30 minutes)

XX1. A Unified Analysis of Fan Stator Noise. Donald B. Hanson, Hamilton Standard, Division of Uniled Aircraft Corporation, I'indsor Locks, Connecticut 06096.-A theory is developed for axial flow fans which predicts the harmonic and broad-band acoustic radiation from the stator due to interaction with rotor viscous wakes. Both the harmonic and broad-band spectrum components are calculated from a unified model using methodology from the theory of random pulse modulation. The stator is modeled as a circular array of pulsed dipoles. The amplitude and arrival time of each pulse are random variables whose means correspond to the values calculated for harmonic rotor-stator interaction theory. The standard deviations of these random variables are measures of the turbulence level in the 
blade wakes. For the model proposed, the solution is exact and the results are instructive: when pulse amplitude modulation (PAM) is imposed on a periodic stator source, new broad-band energy is generated whose spectrum shape is similar to the envelope of harmonics at high frequencies. The harmonic radiation is unchanged. When pulse position modulation (PPM) occurs, new broad-band energy is radiated but at the expense of harmonic energy. The theoretical results are discussed with reference to directivity patterns and radiation efficiency, and the harmonic component is compared with the previous Lowson and Embleton/Theissen analyses. The transfer of harmonic to random energy through PPM is discussed. At frequencies significantly above duct cutoff, it is shown for a fixed stator solidity that the spectrum is essentially independent of the number of stator vanes. In order to show the capabilities of this new theory, calculations are compared in detail with experimental data and modulation levels are discussed.

\section{Contributed Papers (10 minutes)}

XX2. Effects of Blade-Vane Spacing on Fan Noise. W. R. Semrau, Detroit Diesel Allison Division of General Motors Corporation, Indianapolis, Indiana 46206.--Definitive experimental knowledge of discrete-frequency noise reduction, which can be obtained in an aircraft turbofan engine through increased axial spacing between the fan rotor and outlet guide vanes, has been lacking. An experimental scale-model research program has been undertaken to fill this void. Using a unique heavy-gas scale-model anechoic test facility, rearward radiated discrete frequency and broad-band noise was investigated for a scale model fan. Data has been obtained for fan tip Mach numbers of 0.7-1.4, and rotor to OGV spacings of $0.13,0.5,1.0$, and 2.0 rotor chords. Discrete frequency noise reductions up to $17 \mathrm{~dB}$ were obtained. At close spacing, discrete frequency reduction was at a rate greater than $6 \mathrm{~dB}$ per double spacing. Relative harmonic content was not influenced by spacing and reduction was independent of rotor speed. No significant change in broad-band noise was observed.

XX3. Test and Evaluation of a Quiet Helicopter Configuration HH-43B. M. A. Bowes, Kaman Aerospace Corporation, Bloomfield, Connecticut 06002.-A series of noise control modifications was made to the HH-43B helicopter. The objective of these modifications was attainment of a $50 \%$ reduction in forward flight (Ayover) octave-band (SPL) signature. Additionally, the effect of each modification was to be evaluated through direct comparison of the acoustic signatures of modified and unmodified configurations. Modifications were made to the aircraft engine, drive, and rotor systems, resulting in substantial reductions in the helicopter noise signature. Significant reductions were achieved in all octave bands of interest, i.e., $63 \mathrm{~Hz}$ to $4 \mathrm{kHz}$, with an average reduction exceeding $8.5 \mathrm{~dB}$. A total of 10 distinct aircraft configurations were tested, and their respective noise control modifications evaluated. Greatest reductions in noise signature resulted from engine and rotor system modifications. Turbine engine noise reductions were obtained through the use of reactively lined inlet and exhaust silencers, which produced substantial reduction of middle- to high-frequency noise components. Rotor system modifications included both blade geometry changes, i.e., increased diameter and chord, modified airfoil section, increased (negative) blade twist rate, etc., and reduced tip speed. These modifications resulted in acoustic signature changes covering the entire frequency spectrum, i.e., $20 \mathrm{~Hz}$ through $10 \mathrm{kHz}$ [Work supported by ARPA-DOD, monitored by Eustis Directorate, USAAMRDL.]

XX4. Application of External Aerodynamic Diffusion to Reduce Shrouded Propeller Noise. R. E. Longhouse, Center for Acoustical Studies, Depurtment of Mechunical and Acrospace Engineering, North Carolina State University, Raleigh, North Carolina 27607. - A major portion of the noise from V/STOL aircraft fan or shrouded propeller systems occurs during the hover mode. External diffusion of the exhaust flow during this mode significantly increases the thrust/power ratio. At tendant with the flow diffusion is a reduction in fan blade aerodynamic loading. The latter effect suggests a potential source of reducing the lan discrete-frequency noise. Results of model shrouded propeller tests to study the noise reduction associated with external diffusion are presented. [NASA is acknowledged for partial sponsorship of this work.]

XX5. Holographic Study of the Disturbances Generating Supersonic Jet Noise. Richard F. Salant, Massachusells Inslitule of Technology, Cambridge, Massachusetts 02139, J. H. T. Wu, McGill University, Montreal, Canada, Hisayukı HaNDA and JERry W. SoRrow, Massachusetls Institute of Technology, Cambridge, Massachusetts 02139. Optical holography has been used to study the noise-generating disturbances within supersonic nitrogen and helium laboratory-scale jets. Mach angle measurements indicate that the average convection velocity of such disturbances is $77 \%$ of the jet velocity for the nitrogen jet in the Mach number range of $2.8-3.4$, and is $67 \%$ of the jet velocity for the helium jet in the mach number range of 1.5-3.0. The length scale of the disturbances in the nitrogen jet has been found to contain a primary peak at $0.20-0.45$ jet diameters, and a secondary peak between $0.45-0.77$ jet diameters. The amplitude of the secondary peak, relative to the primary peak, increases in the downstream direction. In addition, the peak length scales shift upwards in the downstream direction. [This work was sponsored by the Department of Transportation.]

* Present address: Borg-Warner Corporation, Roy C. Ingersoll Research Center, Des Plaines, IIl. 60018.

XX6. Estimates of the Contribution to Jet Engine Exhaust Noise Made by Internal Sources. E. G. Plett and M. SumMERFIEld, Aerospace and Mechanical Sciences Department, Princelon University, Princelon, New Jersey 08540.-The flow unsteadiness generated inside a turbojet engine by unsteady flow interactions with struts as well as the unsteadiness generated by nonsteady combustion processes may be significant sources of noise in high-bypass aircraft engines. By treating the jet exhaust plane as a surface bounding the otherwise free jet, a surface integral of the appropriate turbulence quantities [N. Curle, Proc. Roy. Soc. 231A, (1955)] over the exit plane of the nozzle yields the contribution from the internal sources. Monopoles, dipoles, and quadrupoles have been used to characterize the fluctuations at the exit plane. Source strengths corresponding to turbulence intensities ranging from $1 \%$ to $20 \%$ are used in calculations. It is shown that if the exit plane and shear region have the same turbulence intensity, then a single exit plane monopole source could produce more noise than $10^{3}$ shear region quadrupoles over the entire subsonic jet Mrch number range. and if the exit plane was filled by a single dipole rather than monopole source, it would be the dominate source to Mach numbers of about 0.6 . [Supported by ONR contract and by NASA grant.] 
XX7. On Protuberance Noise Environments. Balla RD W. GEorge, Lockheed Missiles and Space Company, Sunnyvale, California 94088.-A review of three-dimensional or local protuberance environments is presented with emphasis on the role of the boundary layer. The boundary layer is observed to participate significantly in the generation or propagation of acoustic disturbances throughout the adjacent region. Deductions on boundary layer quantitative effects are made based on data presented by (1) Robertson (1969) and (2) various other investigators. These data are respectively associated with relatively high and low (or roughness) regimes of proturberance height with respect to the boundary layer thickness. Insights are also obtained from the data on twodimensional and axisymmetric steps contributed by various researchers in the past decade. Highest fluctuating pressures, forward and aft of cylindrical protuberances, typically occur in cases where protuberance height is on the order of 1.0-2.0 boundary layer thicknesses. Cylindrical protuberance configurations, having a minimum height $(h)$ of 0.125 boundary layer thickness $(\delta)$, caused a local increase in rms fluctuating pressures (relative to undisturbed case) of 4.0 or more in all cases tested. Comments are included on roughness $(h / \delta<0.1)$ noise sources and amplitudes as a function of height and distribution parameters.

XX8. Further Studies on Cross Correlations between Fluid Dilatations and Flow Noise. R. RACkL, Deparlment of $\mathrm{Me}$ chanical Engineering, Universily of British Columbia, Vancouver 8. Canada.-Following Ribner's dilatation model of turbulent flow, the fluctuating pressures in a jet are considered the sources of sound radiation. Cross correlation between the jet pressure and the noise in the farfield yields the distribution and character of the sound sources in the flow. From the correlation-function, the acoustic source strength, and by applying a Fourier transform, the frequency spectrum of the sound radiated per unit volume of turbulence are obtained. Errors in pressure measurement due to turbulent velocities interacting with the pressure sensor are discussed. It is shown that a cylindrical probe is inadequate for pressure measurements in the present case because of additional dipole noise generated by the turbulent flow impinging on the probe and thereby contaminating the cross correlation. Both the above difficulties are considerably reduced by a foil-type pressure sensor; its design is discussed in detail. The distribution of noise sources in a subsonic jet as they are radiating in a direction of $45^{\circ}$ to the jet axis and the frequency spectra associated with these sources are presented.

XX9. Noise of High Speed Jets with Rough Combustion. A. N. Abdelhamid, D. T. Harrje, E. G. Plett, and M. Summerfielo, Aerospace and Mechanical Sciences Department, Princelon University, Princeton, New Jersey 08540.-An experimental program to demonstrate the effect of unsteady combustion processes in a combustion-augmented jet flow is described. Characteristics of noise for several combustors, having different flame holders and several lengths, operating in a jet temperature range from $1100^{\circ} \mathrm{C}$ to $1500^{\circ} \mathrm{C}$ and a jet Mach number range from 0.2 to 0.35 (jet exit velocity ranging between 400 and $800 \mathrm{ft} / \mathrm{sec}$ ) are presented. Data were collected from six $B \& K \frac{1}{4}$-in. microphones positioned at an angular separation of $15^{\circ}$ in a quadrant of $50-\mathrm{ft}$ radius from the 2-in. diam nozzle exit. Kistler type 601 pressure transducers are mounted in the duct wall of the combustor which is beld on a pylon $12 \mathrm{ft}$ above the ground. The experimental data collected and analyzed to date shows a directivity pattern with a nearly circular pattern, which has a peak in the vicinity of $45^{\circ}$ to $60^{\circ}$ from the jet axis. Comparison of internal pressure fluctuations with the farfield noise shows many simularities in the spectrum, an observation which is verified by the strong cross correlation between the two signals. Combustor and flame holder geometry are found to affect the level and frequency content of the noise. [Supported by NASA grant.]
XX10. Low Frequency Noise of Gas Turbines. R. M. Hoover, Bolt Beranek and Newman Inc., 50 Moulton Street, Cambridge, Massachusetts 02138. - With many electric utility gas turbine installations, the most difficult noise problem has been the control of low-frequency noise radiated to the community from the turbine exhaust. In this paper a number of case histories are discussed leading to some suggestions for noise control design goals for the $31.5-\mathrm{Hz}_{2}$ octave band. Some material will be presented on noise spectra, noise control measures, and noise specifications for gas turbines pertinent to low frequencies.

XX11. Reducing Blade Tonal Noise Generated by VaneAxial Fans. J. V. Pierpoint, Naval Ship Research and Development Center, Annapolis, Maryland 21402.-An experimental investigation was made to determine the eflectiveness of unequally spaced blades and blade/vane interaction techniques in reducing the pure-tone aerodynamic noise generated by vane-axial fans. Balanced mirror-image and other modulated blade patterns were investigated for 13,19 , 20 , and 21 blades in combination with nine equally spaced downstream stator vanes. In-duct discrete-frequency noise analyses were obtained. Blade-passing frequency and its harmonics were modulated and reduced 10-20 dB, using unequally spaced blades. Large spacing deviation had the effect of spreading the sound energy to more harmonics of rotational frequency. In some instances, significant downstream stator vane and upstream strut frequencies were observed. Predictable pure-tone multiples of rotational frequency were also detected. Elimination of inlet strut frequency and large reductions at the harmonics of blade-passing frequency were obtained upon replacement of the inlet bell and stationary nose cone and eight support struts with an inlet bell and spinning nose cone. Unequally spacing the blades of the Ian had little effect on the ability of the fan to move air.

XX12. AMRL Dynamic Pressure Chamber. Rogert G. Powell, 6570th Aerospace Medical Research Laboratory, Wrighl-Palterson AFB, Ohio 45433.-The A.MRL dynamic pressure chamber is an infrasonic simulator expressly designed for biomedical research. This device simulates the low-frequency $\left(0.5-30 \mathrm{H}_{2}\right)$ noise spectrum associated with jet aircraft and rocket launches. Being an electrohydraulic device, it can simulate periodic, random, or impulsive types of environment. This wide dynamic range simulator (104-172 dB SPL) is characterized in terms of its frequency response, linearity, and distortion. Additional information concerning salety circuits, subject accommodation, and mechanical design are also presented.

XX13. Measurements of the Wall Pressure Field in a Transition Boundary Layer on a Flat Plate. F. C. DeMetz, M. J. Casarella, and D. W. Jorgensev, Naval Ship Research and Development Center, Bethesda, Maryland 20034-Experiments have been conducted in the new anechoic flow facility at the Naval Ship Research and Development Center on the How noise features of the transition boundary layer on a flat plate. Measurements were made of the pressure fluctuations in the intermittent region of a naturally developing transition boundary layer over a $14 \mathrm{ft}$ long flat plate in a mild accelerated flow. Results are reported of the power and cross-spectral density of the pressure field of the intermittent turbulent spots, as a function of the intermittency factor. The average burst length, burst frequency, and burst convection velocity are also computed from the data and compared with those of related studies.

XX14. Response of Orthogonally Stiffened Plates to Jet Noise. G. Sen Gupta, Struclures Division, NASA-Langley Research Center, Hamplon, Virginia 23365.-Depending upon the spatial correlation of the noise pressure field and the frequency 
of excitation, the dynamic behavior of the stringers, frames, and the interaction of the adjacent panels considerably affect the vibrations of the attached skin in an aircraft fuselage structure. This problem has been studied by analyzing an infinite plate stiffened by two sets of stiffeners at regular intervals and at right angles to each other. These stiffeners represent the frames and stringers of the fuselage. The jet noise is represented by a random pressure field with a bandlimited white noise spectrum, traveling along the structure in an arbitrary direction with a given convection velocity. Initially, propagation of plane flexural waves in the structure has been studied. The effect of changing the direction of propagation of these waves has been observed. The existence of frequency zones of free propagation has been predicted.
The bandwidth of these zones varies with direction of propagation and certain "preferred" directions of propagation, along which the bandwidth reaches maximum values, have been identified. The effect of the external noise pressure field on the structure has been studied next, to predict the response spectrun and the rms response. The effects of different convection velocities at various directions of propagation have been studied. The peak response is largest when the pressure field wave pattern matches with the structural wave pattern and the direction of propagation of the pressure field coincides with one of the "preferred" directions of propagation of the flexural waves. The method is also used to predict the natural frequencies and the response of a skin-stringer structure, taking the frame stiffnesses into account.

Friday, 1 December 1972

DOMINION ROOM, 2:00 P.M.

\title{
Session YY. Architectural Acoustics III : Sound Transmission Loss and Acoustical Impedance; Theory and Measurement
}

\author{
Kenward S. Oliphant, Chairman
}

657 Howard Street, San Francisco, California $9+105$

\section{Contributed Papers (12 minutes)}

YY1. A Simplified Theory for Predicting the Transmission Loss Characteristics of Double-Panel Constructions. BEN H. Sharp, Research Staff, IVyle Laboratories, 128 Maryland Street, El Segundo, California 90245.-One of the problems in attempting to predict the transmission loss of a double-panel construction has always been the rather cumbersome analytical expression that is involved in the calculation. However, even if the calculation is simplified by the use of a computer, the resulting values of ten bear little resemblance to the measured values. It has long been realized that the disagreement is a result of mechanical connections between the individual panels and the presence, or absence, of absorption material in the cavity, neither of which are taken into account in the classical theory. By simplifying the general acoustical characteristics of double panels, it is shown that it is possible to include these two factors and accurately calculate the transmission loss with the aid of simple expressions. The simplicity of the solution makes it possible to develop optimum interpanel connections and panel configurations using materials of different mechanical properties and to design constructions to meet specific acoustical criteria. [This work was conducted under contract to the U. S. Department of Housing and Urban Development.]

YY2. The Effect of Studs and Cavity Absorption on the Sound Transmission Loss of Plasterboard Walls. Al.AN RINSKy and RichaRd H. Lyon, Department of Mechanical Engineering, Massachusetts Institute of Technology, Cambridge, Massachusetts 02139.- The experimental transmission loss (TL) of a 9-ft $\times 14-$ It single leaf $\frac{1}{2}$-in.-thick plasterboard wall with and without studs compares well with mass law below its critical frequency $(3150 \mathrm{~Hz})$ and is dependent on panel resonance level, as measured with an accelerometer, above the critical frequency. The addition of $2 \times 4$ wood studs every 16 in. to the single panel increased its resonance radiation efficiency below critical frequency by an amount predicted by the ratio of panel impedance to stud lineal mass impedance. By contrast, the addition of steel channel studs every 24 in. had no significant effect on the panel radiation efficiency. Tests on a double leaf plasterboard wall separated $3 \frac{5}{3}$ in. by steel channel studs every 24 -in. showed that the intermediate $\mathrm{TL}$ from source room to the cavity, as determined with a microphone suspended in the cavity, is quite similar to the TL of a single panel between two large rooms.
The cavity is shown to behave as an intermediate resonant system between the transmission and receiver rooms. The cavity loss factor, as determined from reverberation times of sound piped into the cavity, could be increased by the addition of felt or foam strips hung inside the cavity. With continued addition of cavity absorption, an upper TI. limit of the double panel is reached when transmission becomes dominated by receiver-room-panel resonant modes due to flanking through the steel studs and about equally as much dominated by nonreverberant direct sound transmission through the cavity, virtually unaffected by cavity absorption. 'This explains why it has been observed that continued increase in cavity absorption beyond a certain point no longer increases the overall TL of a double leaf wall.

YY3. The Effect of Room Size on Field Sound Transmission Loss Measurements. LUDWig $W^{\text {: }}$. SEPMEYER, Consulting Engineer, Los Angeles, California 90025.-As part of the qualification testing and quality control program for the University of California Residential Building Systems (URBS) project, a field test mockup building was constructed. This facility was designed so that the test walls, having nominal ratings of $\mathbf{4 0}$ and 50 STC, could be measured in three different room sizes similar to those of future dormatories without disturbing the test wall to effect the change in room size. The results of the measurements will be shown and the differences discussed.

YY4. Simplified Field Measurement of Noise Reduction between Spaces. T. L. Quindry and D. R. FlyNN, National Bureau of Standards, Washington, D.C. 20234.-The present ASTM procedure for field measurement of the noise reduction between spaces is time consuming and costly because it requires numerous measurements in order to compute the noise isolation class (NIC), particularly when separate measurements must be made at multiple microphone positions to obtain adequate spatial averaging. The purpose of the present investigation is to explore the relationships between $\mathrm{NIC}$ values based on $\frac{1}{3}$-oct band data and more easily obtained ratings of isolation based on either octave band data or on $A$-weighted or $C$-weighted sound level data. A large number of curves of $\frac{1}{3}$-oct band noise reduction versus frequency were calculated from data on transmission loss of partitions, assumed room geometry, assumed values for the source sound 
power spectrum, and assumed values for the sound absorption in the source and receive rooms. For each of these $\frac{1}{3}$-oct band noise reduction curves, the following quantities were calculated and compared: NIC rating, $I_{a}$ rating ( $\frac{1}{3}$-oct band data), $I_{a}$ rating (octave band data), the $A$-weighted sound level difference, and the difference between the $C$-weighted level in the source room and the $A$-weighted level in the receive room.

YY5. Transmission of Sound through Enclosures. C. I. Holmer, Bolt Beranek and Neruman Inc., 50 Moulton Street, Cambridge, Massachusetts 02138 . - The exact description of sound transmission through an enclosure depends, in general, on the details of the source radiation chardcteristics, coupling of the source to the enclosure acoustic space, and coupling of the acoustic space to the resonant and nonresonant moticn of the enclosure walls. Moreover, for an extended source, and a relatively small and absorptive enclosed space, an exact description is beyond the present state of the art. In this paper, a simple design procedure based on an energy flow analysis is described, which provides good lower bound estimates of enclosure performance. The performance parameter that is calculated is the lower bound of a space average, or radiated power insertion loss. When formulated in this way, the performance is reciprocal in the sense that it is independent of whether transmission is from outside to inside or inside to outside. Above the first enclosure volume resonance, the result is PIL $\geq \mathrm{TL}+10 \log A / S$ where PIL is the power insertion loss, $\mathrm{TL}$ is the field incidence transmission loss of the enclosure walls, $A$ is the sabine absorption of the enclosure interior, and $S$ is the radiating area of the walls. A result that is applicable below the first enclosure resonance is also presented. A number of experimental results are presented which confirm the analysis procedures. [Work supported in part by General Electric Co., Aircraft Engine Group, Cincinnati, Ohio, and NASA-Langley Research Center.]

YY6. Acoustic Impedance Measurements for Various Angles of Incidence. Gkegory C. Tocci and Huw G. Davies, Department of Mechanical Engineering, Massachusetts Instilute of Technology, Cambridge, Massachusetts 02139.-A simple correlation technique is presented for measuring acoustic absorption coefficients and impedances of surfaces for sound incident at any angle. The measurements can be made in situ; the technique is useful for measuring ground impedance and the surface impedance of buildings, as well as the impedance of acoustic absorbing material. The technique uses a simple source and one microphone, both placed a few acoustic wavelengths away from the surface. The source signal is random noise, and the microphone output is filtered through a narrow frequency band with a suitably smooth filter shape. Values of the absorption coefficient and impedance at any frequency are obtained from the autocorrelation curve of this narrowband signal. [Work supported by NSF.]

YY7. Theoretical Considerations in the Determination of Complex Acoustical Impedance of Nonlocally Reacting Noise Control Materials. ARUN G. JHAVERI, Harris F. Freedman \&o Associates, Mercer Island, W'ashington 98040.-Various techniques and computer programs have been developed for the determination of complex acoustic impedance of thin (locally reacting) noise-control materials, such as metal felt, polyimide, perforated plate, etc., within last five years. However, there is very little published data available for the calculation of complex impedance and absorption coefficient of thick, homogeneous, and nonlocally reacting noise control materials, such as glass fiber and open-pore polyurethane foam. The present paper establishes theoretical guidelines for the computation of acoustical parameters that determine the complex acoustical impedance of porous, homogeneous, and isotropic materials. These materials have been and are being used for the abatement and control of air-conditioning and air-borne types of environmental noise. A thick or nonlocally reacting type of material is characterized by two complex numbers, namely, the characteristic impedance $(z / \rho c$ or $w)$, and the propagation constant $(\gamma)$, as opposed to only one complex number for thin or locally reacting materials. Then, two separate measurements based on theoretical equations contained herein yielding two complex numbers, would suffice in the determination of required complex acoustic impedance and absorption coefficient as a function of discrete frequency $f$.

YY8. Acoustic Measurements Using Acoustic Pulse Trains of Infinite Duration. Richard E. VAN DoEren, North Star Research and Development Institute, 3100 38th Avenue South, Minneapolis, Minnesota 55406.-Many workers in the field of acoustics have found that measurements must be performed in nonideal environments in which error contributions from reflections and diffractions can be significant. Furthermore, creation of satisfactory acoustical measurement conditions (e.g., anechoic or reverberant rooms) can be costly both in dollars and space. Use of acoustic pulsetrains of infinite duration has definite advantages for measurement of reflection and transmission coefficient of achitectural panels and for measurement of sound pressure level effects of full-size or scaled noise reduction systems. The experimental pulse system at North Star is described and application of pulsetrain techniques to measurement of reflection, transmission, and sound-pressure-level reduction of scale- and full-size systems is discussed. Experimental results are given for transmission and reflection from architectural-type panels and for a scale model of a highway barrier.

YY9. Comparison of Reverberation-Room and Impedance Tube Measurements on Carpets. Maurice Poulin and T. D. Northwood, Dirision of Building Research, National Research Council of Canada, Otlawa, K1A OR6, Canada.-Carpet is a material difficult to define in terms that relate to its sound absorbing capacity. Accordingly there is use for a simple method of measuring its sound absorption. The reverberation room method (ASTM C423) provides the definitive measure for random incidence absorption when large specimens are available. The impedance tube method requires only small specimens but measures normal-incidence absorption, which is not a useful measure of performance in rooms. For some materials, deemed "locally reacting" with respect to the incident sound, one may calculate random-incidence absorption from tube data, but this cannot be assumed, especially for a loose ensemble of components such as carpet. Accordingly the correspondence between the two methods of measurement was studied for a range of carpet specimens. Results indicate that calculations from impedance tube data, corrected according to the locally reacting assumption and taking account of diffraction effects in the reverberation room, yield lower absorption coefficients than are obtained directly in the reverberation room. Since a good correspondence is found with truly locally reacting materials, it is inferred that carpet does not thus qualify.

YY10. Operational Methods in Determining the Acoustical Green's Function of a Right Parallelepiped with Perfectly Reflecting Walls. J. A. Selvaggi, Gannon College, Erie, Pennsylvania 16501.-The rules of LaPlace transforms are involved in the solution of

$$
\nabla^{\prime \prime} \phi-\frac{1}{v^{2}} \frac{\partial^{2} \phi}{\partial \ell^{2}}=\delta(x-a) \delta(y-b) \delta(z-c) \delta(l) .
$$

The boundary conditions of a perfectly reflecting wall are applied. The solution displays the effect of the position of the source on the amplitude response. The acoustic potential is determined by $\bar{V}=-\nabla \phi$. Real convolution extends the solution to any time dependent input. 